Universidade de São Paulo

Instituto de Física

\title{
Estrutura de Cluster-alfa em Núcleos da Região do Molibdênio
}

\author{
Marco Antonio de Souza
}

Orientador: Prof. Dr. Hideaki Miyake

Tese de doutorado apresentada ao Instituto de Física para a obtenção do título de Doutor em Ciências.

Banca examinadora:

Prof. Dr. Hideaki Miyake (IFUSP)

Prof. ${ }^{a}$ Dr. ${ }^{a}$ Alinka Lépine (IFUSP)

Prof. Dr. Celso Luiz Lima (IFUSP)

Prof. Dr. Sérgio José Barbosa Duarte (CBPF)

Prof. Dr. Nilton Teruya (UFPB) 


\section{FICHA CATALOGRÁFICA}

Preparada pelo Serviço de Biblioteca e Informação do Instituto de Física da Universidade de São Paulo

Souza, Marco Antonio de

Estrutura de Cluster-alfa em núcleos da região do Molibdênio. São Paulo, 2010.

Tese (Doutorado) - Universidade de São Paulo. Instituto de Física - Depto. de Física Experimental

Orientador: Prof. Dr. Hideaki Miyake

Área de Concentração: Física

Unitermos: 1. Física nuclear; 2. Estrutura nuclear;

3. Modelo nuclear. 


\section{Agradecimentos}

Ao Prof. Hideaki Miyake, pelo incentivo dado a mim durante todo o projeto e pelas importantes discussões que contribuíram para o desenvolvimento deste trabalho.

À Prof. ${ }^{a}$ Thereza Borello-Lewin e aos demais membros do Grupo de Espectroscopia Nuclear com Íons Leves do IFUSP, pela oportunidade de convivência que favoreceu o amadurecimento do meu trabalho e pelo incentivo dado a este projeto.

Aos meus pais Adivair e Ademar, e aos meus irmãos Carlos, Douglas e Adriana, por todo o apoio e incentivo que recebi durante a minha vida estudantil.

A Leny Florêncio da Silva, por todo o apoio dado a mim nos momentos de sucessos ou dificuldades, além de suas importantes sugestões na revisão gramatical do texto.

À CAPES, pela bolsa concedida para a realização deste trabalho. 


\section{Sumário}

$\begin{array}{ll}\text { Resumo } & 4\end{array}$

$\begin{array}{ll}\text { Abstract } & 5\end{array}$

1 Características do modelo de cluster- $\alpha \quad 6$

1.1 Introdução . . . . . . . . . . . . . . . . . . . 6

1.2 Descrição do modelo de cluster- $\alpha \ldots \ldots$. . . . . . . . . . . 9

1.3 Potencial de interação do sistema $\alpha+$ caroço . . . . . . . . . . . . . 11

2 Processo de seleção dos núcleos estudados $\quad 16$

2.1 Critério de seleção dos núcleos com estrutura $\alpha+$ caroço . . . . . . 16

2.2 Avaliação preliminar do critério de $Q_{\alpha} / A_{T} \ldots \ldots$. . . . . . . . . 19

2.3 Seleção dos núcleos preferenciais para a aglomeração- $\alpha$ na região do

Molibdênio . . . . . . . . . . . . . . . . . 25

3 Determinação das bandas de estado fundamental dos sistemas $\alpha+$ caroço $\quad 32$

3.1 Descrição inicial do sistema $\alpha+$ caroço . . . . . . . . . . . . . . . 32

3.2 Método numérico aplicado à resolução do sistema . . . . . . . . . . . 33

3.3 Tratamento para estados quase-ligados . . . . . . . . . . . . . . . 37

3.4 Cálculo das bandas de estado fundamental . . . . . . . . . . . . . . 38

3.4.1 Identificação do número quântico $G$ para os sistemas $\alpha+$ caroço . . . . . . . . . . . . . . . . 38

3.4.2 Ajuste do parâmetro $R$ para os sistemas $\alpha+$ caroço . . . . . 39

3.5 Resultados para as bandas de estado fundamental . . . . . . . . . . 40

3.6 Aspectos referentes aos níveis de energia experimentais . . . . . . . 44

3.7 Normalização das funções radiais $u(r)$. . . . . . . . . . . . . . . . . . . . . . . . . . . . . . . 45

3.8 Funções radiais normalizadas $u_{N, L}(r) \ldots \ldots$. . . . . . . . . . . 46

Observação sobre a implementação dos métodos numéricos . . . . . . . . 50 
4 Propriedades das bandas de estado fundamental dos sistemas $\alpha+$ caroço

4.1 Comportamento do parâmetro $V_{0}$ do potencial $\alpha+$ caroço . . . . . . 51

4.1.1 Variação de $V_{0}$ em relação ao valor padrão de $220 \mathrm{MeV}$. . . 51

4.1.2 Comportamento do parâmetro $V_{0}$ em relação ao número quântico $L$. . . . . . . . . . . . . . . . . . . . . . 52

4.1.3 Comportamento do parâmetro $V_{0}$ em outras regiões de massa 58

4.2 Comportamento do parâmetro $R$ do potencial $\alpha+$ caroço . . . . . . 61

4.2.1 Análise do parâmetro $R$ na região do Molibdênio . . . . . . 61

4.2.2 Análise do parâmetro $R$ em diferentes regiões de massa . . . 63

4.3 Separações intercluster rms . . . . . . . . . . . . . . . . . 66

4.4 Larguras- $\alpha$ reduzidas . . . . . . . . . . . . . . . . . . . . . . . . . . 69

4.4.1 Definições referentes à largura reduzida . . . . . . . . . . . . 69

4.4.2 Critério para a escolha do raio de canal . . . . . . . . . . 71

4.4.3 Resultados para as larguras- $\alpha$ reduzidas na região do Molibdênio . . . . . . . . . . . . . . . . 72

4.4.4 Comportamento das larguras- $\alpha$ reduzidas em outras regiões de massa . . . . . . . . . . . . . . . . . . . . . . . 73

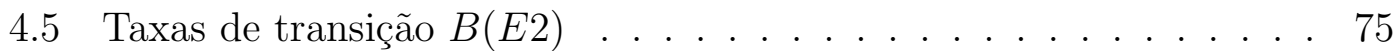

5 Procura por bandas de paridade negativa nos sistemas $\alpha+$ caroço 80

5.1 Exemplos na literatura sobre bandas de paridade negativa . . . . . 80

5.2 Bandas de paridade negativa nos núcleos da região do Molibdênio . 81

5.2.1 Aspectos específicos do cálculo das bandas de paridade negativa . . . . . . . . . . . . . . . . 8 81

5.2.2 Procedimento específico para a determinação de níveis de energia ressonantes . . . . . . . . . . . . . . . 82

5.2.3 Resultados obtidos para as bandas de paridade negativa ....................... 83

5.3 Aspectos referentes aos níveis de energia experimentais . . . . . . . 86

$\begin{array}{lr}\text { Conclusões e perspectivas } & 89\end{array}$

A Determinação das funções $V_{0}(L)$

A.1 Adaptação da função $y_{1}(x)=a_{1} x^{3}+b_{1} x^{2}$ para a descrição do parâmetro $V_{0} \ldots \ldots \ldots \ldots \ldots \ldots$

A.2 Adaptação da função $y(x)=\exp \left(a_{2} x\right) / \exp \left(b_{2} x^{3}\right)$ para a descrição do parâmetro $V_{0} \ldots \ldots \ldots \ldots$. . . . . . . . . . 95 
B Estrutura de cluster- $\alpha$ no ${ }^{13} \mathrm{C}$

B.1 Descrição do artigo "Simple description of alpha-cluster states in ${ }^{13} \mathrm{C} " \ldots \ldots \ldots \ldots$. . . . . . . . . . . . . . . . . 98

B.2 Descrição do artigo "Investigation of $\alpha$-cluster states in ${ }^{13} \mathrm{C}$ via the $\left({ }^{6} \mathrm{Li}, \mathrm{d}\right)$ reaction" . . . . . . . . . . . . . . . . . . . . . . . . . . 98

Referências Bibliográficas 


\section{RESUMO}

O modelo de cluster- $\alpha$ é aplicado aos núcleos de massa intermediária ${ }^{90} \mathrm{Sr},{ }^{92} \mathrm{Zr},{ }^{94} \mathrm{Mo},{ }^{96} \mathrm{Ru}$ e ${ }^{98} \mathrm{Pd}$ com a Abordagem de Potencial Local. As bandas do estado fundamental dos respectivos sistemas $\alpha+$ caroço são calculadas com um único parâmetro variável, fornecendo uma boa descrição geral dos níveis experimentais. Mostra-se que o potencial $\alpha+$ caroço é fracamente dependente do momento angular $L$, e que tal dependência pode ser descrita de forma simples e padronizada para os cinco núcleos. O comportamento do parâmetro radial $R$ do potencial $\alpha+$ caroço é discutido em relação ao raio do núcleo total e a soma dos raios do cluster- $\alpha$ e do caroço. As taxas de transição $B(E 2)$ reproduzem corretamente as ordens de grandeza de quase todos os dados experimentais sem o uso de cargas efetivas. A análise das separações intercluster rms e das larguras- $\alpha$ reduzidas nas bandas do estado fundamental sugere que há uma redução da intensidade de aglomeração- $\alpha$ com o aumento do spin. Uma análise complementar das bandas de estado fundamental dos núcleos ${ }^{20} \mathrm{Ne},{ }^{44} \mathrm{Ti}$ e ${ }^{212} \mathrm{Po}$ aponta uma diminuição da intensidade de aglomeração- $\alpha$ com o aumento da massa nuclear, e mostra uma condição mais fraca de aglomeração- $\alpha$ para os núcleos da região do Mo em comparação com os núcleos leves. Bandas de paridade negativa são calculadas para os núcleos ${ }^{92} \mathrm{Zr},{ }^{94} \mathrm{Mo},{ }^{96} \mathrm{Ru}$ e ${ }^{98} \mathrm{Pd}$ e informações não relatadas anteriormente são comparadas a níveis experimentais disponíveis. A avaliação geral dos resultados indica que os núcleos com $A$ par e $N=52$ na região do Mo possuem estruturas $\alpha+$ caroço com características semelhantes. 


\begin{abstract}
The $\alpha$-cluster model is applied to the intermediate mass nuclei ${ }^{90} \mathrm{Sr}$, ${ }^{92} \mathrm{Zr},{ }^{94} \mathrm{Mo},{ }^{96} \mathrm{Ru}$ and ${ }^{98} \mathrm{Pd}$ with the Local Potential Approach. The ground state bands of the respective $\alpha+$ core systems are calculated with only one variable parameter, giving a good general description of the experimental data. It is shown that the $\alpha+$ core potential is weakly dependent on the angular momentum $L$ and such dependence may be described in a simple and standardized form for the five nuclei. The behavior of the radial parameter $R$ of the $\alpha+$ core potential is discussed in relation to the radius of the total nucleus and the sum of the radii of the $\alpha$-cluster and the core. The calculated $B(E 2)$ transition rates reproduce correctly the orders of magnitude of almost all experimental data without the use of effective charges. The analysis of the rms intercluster separations and the reduced $\alpha$-widths for the ground state bands suggests a reduction of the $\alpha$-cluster intensity with the increasing spin. A complementary analysis of the ground state bands of the nuclei ${ }^{20} \mathrm{Ne},{ }^{44} \mathrm{Ti}$ and ${ }^{212} \mathrm{Po}$ points to a decrease of the $\alpha$-cluster intensity with the increasing nuclear mass, and shows a weaker $\alpha$-cluster condition for the nuclei of the Mo region in comparison with the light nuclei. Negative parity bands are calculated for the nuclei ${ }^{92} \mathrm{Zr},{ }^{94} \mathrm{Mo},{ }^{96} \mathrm{Ru}$ and ${ }^{98} \mathrm{Pd}$ and previously not reported information are compared to available experimental levels. The general evaluation of the results indicates that the $N=52$ even- $A$ nuclei in the Mo region have $\alpha+$ core structures with similar features.
\end{abstract}




\section{Capítulo 1}

\section{Características do modelo de cluster- $\alpha$}

Este capítulo descreve as características do modelo de aglomerado- $\alpha$ (cluster- $\alpha$ ) e dos aspectos específicos para a sua aplicação aos núcleos da região do Molibdênio. Uma introdução discute vários exemplos da literatura sobre o modelo e as motivações para o seu uso na região de interesse.

\subsection{Introdução}

A estrutura de cluster- $\alpha$ é uma característica importante na interpretação de dados espectroscópicos de núcleos leves. O modelo de cluster- $\alpha$ mostrou êxito na reprodução de vários níveis dos espectros de energia, propriedades eletromagnéticas, larguras de emissão- $\alpha$ e dados referentes ao espalhamento elástico de partículas- $\alpha$ em núcleos próximos aos de dupla camada fechada, tais como ${ }^{16} \mathrm{O}$ e ${ }^{40} \mathrm{Ca}$. Diante dos resultados favoráveis obtidos para os núcleos leves, o mesmo modelo foi aplicado nos trabalhos que se sucederam, estes citados e comentados nesta seção, na região de núcleos meio-pesados e pesados, indicando que a estrutura de cluster- $\alpha$ mantém um comportamento similar ao encontrado em núcleos leves.

O modelo de cluster propõe que certos núcleos apresentam uma condição favorável para o surgimento de estados associados a movimentos coletivos de dois ou mais agrupamentos de núcleons, constituindo um sistema de aglomerados no núcleo total. Tal sistema corresponde à chamada estrutura de cluster do núcleo. Esta interpretação representa uma alternativa à descrição de estados nucleares que poderiam ser investigados, em princípio, por tratamentos microscópicos mais complicados.

A configuração de estrutura binária $\alpha+$ caroço ocorre preferencialmente em 
núcleos onde o cluster- $\alpha$ é formado por núcleons em orbitais acima de camada(s) fechada(s) e/ou de subcamada(s) completa(s). Um método simples para a análise do sistema $\alpha+$ caroço e outros sistemas cluster + caroço baseia-se na Abordagem de Potencial Local (APL), onde todas as propriedades são obtidas através de um potencial local de interação cluster + caroço. Desta forma, os estados de partícula única produzidos por este potencial são usados para o cálculo de níveis de energia, larguras de decaimento- $\alpha$, taxas de transição eletromagnéticas e outras propriedades.

Os próximos parágrafos desta seção discutem exemplos na literatura de aplicações da APL para sistemas $\alpha+$ caroço em diferentes regiões de massa e os sucessos alcançados.

Conforme o princípio descrito anteriormente, a estrutura de cluster- $\alpha$ foi estudada em detalhes nos núcleos ${ }^{16} \mathrm{O},{ }^{20} \mathrm{Ne}$ e ${ }^{44} \mathrm{Ti}$, considerando respectivamente os sistemas $\alpha+{ }^{12} \mathrm{C}, \alpha+{ }^{16} \mathrm{O}$ e $\alpha+{ }^{40} \mathrm{Ca}$. O trabalho de Buck, Dover e Vary [1] sobre os núcleos ${ }^{16} \mathrm{O}$ e ${ }^{20} \mathrm{Ne}$ e o trabalho de Michel, Reidemeister e Ohkubo [2] sobre o ${ }^{44} \mathrm{Ti}$ mostram resultados que demonstram a eficiência do modelo de cluster- $\alpha$ com APL na reprodução do comportamento geral das primeiras bandas de níveis experimentais dos três núcleos. O mesmo modelo também foi usado para a determinação das taxas de transição $B(E 2)$ entre os níveis das bandas calculadas, havendo boa concordância com dados experimentais sem a necessidade de altas cargas efetivas. Em contraposição, os valores de $B(E 2)$ calculados através do modelo de camadas para os três núcleos encontram-se mais afastados dos dados experimentais, e para que se aproximem, altas cargas efetivas são necessárias. As larguras de decaimento- $\alpha$ calculadas para os núcleos ${ }^{16} \mathrm{O}$ e ${ }^{20} \mathrm{Ne}$ [1] fornecem uma boa reprodução das ordens de grandeza das respectivas larguras experimentais. O trabalho posterior de Buck, Merchant e Perez [3], que utiliza outra forma de potencial de interação $\alpha+$ caroço, confirmou os resultados favoráveis do modelo para os núcleos ${ }^{20} \mathrm{Ne}$ e ${ }^{44} \mathrm{Ti}$.

Os sucessos obtidos para os núcleos ${ }^{16} \mathrm{O},{ }^{20} \mathrm{Ne}$ e ${ }^{44} \mathrm{Ti}$ motivaram a procura por estados de cluster- $\alpha$ em núcleos mais pesados. Seguindo o princípio de escolha de núcleos com a estrutura $\alpha+$ (caroço de dupla camada fechada), trabalhos posteriores foram publicados sobre a estrutura de cluster- $\alpha$ em ${ }^{94} \mathrm{Mo}$ e ${ }^{212} \mathrm{Po}$, analisando respectivamente as propriedades dos sistemas $\alpha+{ }^{90} \mathrm{Zr}$ e $\alpha+{ }^{208} \mathrm{~Pb}$. Sobre o ${ }^{94} \mathrm{Mo}$, o trabalho de Buck, Merchant e Perez [3] descreve a estrutura $\alpha+{ }^{90} \mathrm{Zr}$ através de um potencial local fenomenológico para a interação $\alpha+$ caroço, onde um conjunto de parâmetros fixos é empregado neste núcleo e em outros de diferentes regiões de massa. Os resultados mostram que o modelo descreve satisfatoriamente os níveis da banda do estado fundamental do ${ }^{94} \mathrm{Mo}$ e reproduz corretamente a ordem de grandeza das taxas $B(E 2)$ experimentais sem o uso de cargas efetivas. O trabalho de Ohkubo [4] também analisa o sistema $\alpha+{ }^{90} \mathrm{Zr}$ através de um potencial do tipo double folding e consegue descrever corretamente a banda do estado fundamental 
do ${ }^{94} \mathrm{Mo}$, com uma fraca dependência do número quântico $L$, além de fornecer uma boa descrição das seções de choque de espalhamento elástico $\alpha+{ }^{90} \mathrm{Zr}$. Ainda na Ref. [4], os valores absolutos das taxas de transição $B(E 2)$ são bem reproduzidos com uma baixa carga efetiva $(\delta e=0.2 e)$. Outros trabalhos no ${ }^{94} \mathrm{Mo}$ de Michel et al. [5] e Souza e Miyake [6] reforçam a presença da estrutura- $\alpha$ nos estados deste núcleo, o mais recente numa linha próxima desta tese.

A respeito do ${ }^{212} \mathrm{Po}$, diferentes trabalhos [3, 4, 7] trataram da estrutura do tipo $\alpha+{ }^{208} \mathrm{~Pb}$ por meio da $\mathrm{APL}$, obtendo resultados similares àqueles do ${ }^{94} \mathrm{Mo}$ para a banda do estado fundamental e as seções de choque de espalhamento elástico $\alpha+{ }^{208} \mathrm{~Pb}$. Adicionalmente, o modelo fornece uma descrição satisfatória das ordens de grandeza das meias-vidas experimentais $T_{1 / 2}$ dos estados da banda.

Estudos da estrutura de cluster- $\alpha$ em núcleos próximos de ${ }^{20} \mathrm{Ne}$ e ${ }^{44} \mathrm{Ti}$ também foram desenvolvidos. Com relação ao ${ }^{20} \mathrm{Ne}$, há exemplos como os núcleos ${ }^{19} \mathrm{~F}$ $[8,9,10],{ }^{18} \mathrm{~F}[9,11,12],{ }^{18} \mathrm{O}[9,11]$ e ${ }^{24} \mathrm{Mg}[13]$, além do núcleo ${ }^{16} \mathrm{O}[1,13]$ já mencionado. Em alguns trabalhos, tais núcleos são tratados por métodos ainda baseados na APL, contudo, necessitando de adaptações para a inclusão de termos adicionais na interação $\alpha+$ caroço associados a forças não-centrais e/ou possíveis excitações internas do caroço. Outros analisam a estrutura de cluster- $\alpha$ através de métodos mais detalhados $[9,13]$ como o Modelo de Condição de Ortogonalidade, o Método do Grupo Ressonante ou a mistura de estados do sistema $\alpha+$ caroço com estados de modelo de camadas. No caso específico do ${ }^{24} \mathrm{Mg}$, mostra-se que há componentes das estruturas $2 \alpha+{ }^{16} \mathrm{O}$ e $\alpha+{ }^{20} \mathrm{Ne}$ em seus estados, além de outras formas de aglomeração. Ao redor do ${ }^{44} \mathrm{Ti}$, diferentes núcleos foram analisados com respeito à estrutura de cluster- $\alpha$, como ${ }^{38,40} \mathrm{Ar}[14],{ }^{40,41,42} \mathrm{Ca}[14,15,16] \mathrm{e}{ }^{42,43} \mathrm{Sc}$ $[15,16]$, utilizando métodos iguais ou semelhantes aos descritos anteriormente.

O trabalho de P. Mohr [17] aborda a estrutura $\alpha+$ caroço de núcleos ao redor do ${ }^{94}$ Mo por meio de um potencial nuclear do tipo double folding para a interação $\alpha+$ caroço. Embora os resultados obtidos sejam favoráveis para as taxas $B(E 2)$ das bandas de estado fundamental, esta referência não apresenta cálculos de outras propriedades do sistema $\alpha+$ caroço e discute muito brevemente a possível existência de bandas de paridade negativa associadas a esta estrutura.

Diante da necessidade de um estudo mais detalhado sobre a estrutura de cluster- $\alpha$ na região de massa intermediária, o presente trabalho mostra uma análise abrangente dos núcleos desta região ao redor do ${ }^{94}$ Mo. Para isto, os sistemas $\alpha+$ caroço são discutidos na perspectiva da APL. Várias propriedades das bandas de estado fundamental são discutidas e evidências a respeito de bandas de paridade negativa são buscadas. Possíveis padrões de comportamento entre os núcleos desta região são investigados. 


\subsection{Descrição do modelo de cluster- $\alpha$}

A aglomeração- $\alpha$ é um caso particular entre as diferentes estruturas de cluster nos núcleos. O modelo de cluster geralmente é aplicado em núcleos cujas propriedades podem ser descritas em termos de sistemas com aglomerados de alto grau de estabilidade. Tal estabilidade é geralmente procurada nos aglomerados que apresentam camadas fechadas ou subcamadas completas de prótons e nêutrons ou que estão próximos da condição de dupla camada fechada, mas outras considerações a respeito da energia de ligação dos aglomerados podem ser usadas para a determinação da estrutura de cluster mais estável num certo núcleo (detalhes no Capítulo 2). Com isso, os trabalhos relacionados às estruturas de cluster frequentemente utilizam a aproximação onde os aglomerados do sistema são tratados como inertes, isto é, sem excitação interna. Nestes casos, os termos relevantes na Hamiltoniana do sistema são aqueles associados aos movimentos dos centros de massa dos aglomerados ou aos movimentos relativos entre os aglomerados.

Os núcleos estudados neste trabalho são descritos em termos da estrutura binária $\alpha+$ caroço, onde os dois constituintes são tratados como inertes. Sabe-se que a partícula- $\alpha$ é um núcleo duplamente mágico, com uma alta energia de ligação por núcleon $(\approx 7.07 \mathrm{MeV} /$ núcleon [18]) em relação a outros núcleos leves e que possui seu primeiro estado excitado acima de $E_{x}=20 \mathrm{MeV}$, favorecendo o seu tratamento como um aglomerado inerte. A interação entre $\alpha$ e caroço é simulada por um potencial local $V(r)$ (mais detalhes na próxima seção), onde $r$ é o raio intercluster (o raio associado à distância entre os centros de massa do cluster- $\alpha$ e do caroço).

Tomando o modelo de camadas como referência, os núcleons constituintes do cluster- $\alpha$ devem se situar em orbitais acima (ou fora) daqueles já preenchidos pelo caroço, respeitando-se o princípio de Pauli. Assim, os números quânticos que caracterizam o movimento relativo $\alpha+$ caroço devem ser compatíveis com esta restrição. Os auto-estados $|N, L, M\rangle$ do sistema cluster + caroço são identificados pelos números quânticos $N, L$ e $M$, onde $N$ é o número de nós internos da autofunção radial (detalhes no Capítulo 3) e $L$ e $M$ são os números relacionados ao momento angular orbital. Uma relação usada frequentemente para os sistemas $\alpha+$ caroço estabelece que

$$
2 N+L=\sum_{i=1}^{A_{c}}\left(2 n_{i}+l_{i}\right),
$$

onde $n_{i}$ e $l_{i}$ são os números quânticos associados ao orbital ocupado pelo $i$-ésimo núcleon constituinte do cluster, sendo $A_{c}$ o número de núcleons do cluster. As definições de $n_{i}$ e $l_{i}$ são análogas às mencionadas respectivamente para $N$ e $L$. 
A equação (1.1) costuma ser mencionada como a Condição de Wildermuth ${ }^{1}$. Esta fórmula é obtida através da relação de equivalência entre a Hamiltoniana dos núcleons individuais, a qual é descrita pelo modelo de camadas de oscilador harmônico e considera $A_{c}$ núcleons de valência ocupando os orbitais fora do caroço, e a Hamiltoniana do sistema cluster + caroço, a qual leva em conta a não excitação interna dos dois aglomerados. Detalhes sobre a construção de tais funções Hamiltonianas e os princípios que originam a eq. (1.1) encontram-se nas Refs. [19] e [20]. O uso de (1.1) permite a determinação aproximativa dos auto-estados do sistema cluster + caroço cuja existência é permitida pelo princípio de Pauli. Os auto-estados do sistema formam conjuntos identificados pelo número quântico global

$$
G=2 N+L
$$

o qual identifica uma banda de níveis de energia. O número de banda $G$ exerce uma função semelhante à do número quântico principal do oscilador harmônico, pois determina a localização média dos níveis da banda dentro do espectro de energia. Considerando $G_{\min }$ como o número quântico global associado à configuração de mais baixa energia dos núcleons do cluster $^{2}$, temos que as bandas fisicamente permitidas para este sistema são aquelas que satisfazem a condição $G \geq G_{\min }$. Exemplos práticos sobre o cálculo de $G_{\min }$ para diferentes sistemas $\alpha+$ caroço são apresentados nas Seções 3.4 e 4.1.

Nos sistemas onde cluster e caroço apresentam spins totais nulos, não são esperados efeitos de forças não-centrais (como a interação spin-órbita) na Hamiltoniana do movimento relativo ${ }^{3}$. Para estes sistemas, os números $G$ pares correspondem a bandas formadas por estados com $J$ inteiro e paridade positiva $\left(0^{+}, 2^{+}, 4^{+}, \ldots\right)$ enquanto os números $G$ ímpares correspondem a bandas formadas por estados com $J$ inteiro e paridade negativa $\left(1^{-}, 3^{-}, 5^{-}, \ldots\right)$.

A equação (1.1) é eficiente na identificação das bandas de estado fundamental de diferentes sistemas de cluster em núcleos leves e meio-pesados, em particular nos sistemas $\alpha+$ caroço. Contudo, a mesma não deve ser entendida como uma condição exata, pois torna-se um tanto imprecisa na determinação dos números

\footnotetext{
${ }^{1}$ Para um sistema cluster + caroço onde o cluster possui $A_{c}>4$, deve-se usar a forma generalizada da Condição de Wildermuth dada por $2 N+L=\sum_{i=1}^{A_{c}}\left(2 n_{i}+l_{i}\right)-G_{\text {int }}$, onde $G_{\text {int }}$ é o número de quanta de oscilador harmônico associado à Hamiltoniana interna do cluster. No caso do cluster- $\alpha$, temos $G_{\text {int }}=0$, já que seus quatro núcleons ocupam internamente a camada $1 s\left(n_{\text {int }}=0\right.$ e $l_{\text {int }}=0$ para os quatro núcleons). Para um cluster de ${ }^{16} \mathrm{O}$, por exemplo, temos $G_{\text {int }}=12$, já que 4 núcleons ocupam internamente a camada $1 s$ e 12 núcleons ocupam internamente a camada $1 p$.

${ }^{2}$ Para os núcleos estudados neste trabalho, a configuração de mais baixa energia é aquela que corresponde ao estado fundamental do núcleo total.

${ }^{3}$ No Apêndice B, é mostrado um tratamento simplificado do sistema $\alpha+{ }^{9} \mathrm{Be}$, onde o caroço de ${ }^{9}$ Be possui spin não nulo.
} 
quânticos $G$ mais apropriados para a descrição de bandas de estado fundamental em núcleos pesados. Esta deficiência se deve aos seguintes aspectos:

- A Condição de Wildermuth baseia-se no modelo de camadas de oscilador harmônico, o qual fornece uma boa aproximação quando os núcleons constituintes do cluster ocupam orbitais de baixo spin. Porém, tal condição perde eficiência quando os núcleons do cluster ocupam orbitais com altos spins, pois o efeito da interação spin-órbita provoca um forte desvio entre os níveis previstos pelo oscilador harmônico e os níveis do modelo de camadas mais realista.

- Para que a eq. (1.1) seja obtida, é necessário considerar que o parâmetro de oscilador harmônico $\hbar \omega$ seja o mesmo para a Hamiltoniana do núcleo total e as Hamiltonianas internas do cluster (ou clusters) e do caroço. Esta aproximação é razoável quando se trata da estrutura de cluster em núcleos leves. No entanto, para regiões de maiores massas nucleares, esta aproximação torna-se mais grosseira, pois sabe-se que $\hbar \omega$ é proporcional a $A^{-1 / 3}$ e pode haver uma grande diferença de massa entre o núcleo total e os aglomerados do sistema.

Portanto, de uma forma geral, a eq. (1.1) serve como um guia estimativo da grandeza do número quântico $G$ associado à banda do estado fundamental de um certo sistema de cluster.

\subsection{Potencial de interação do sistema $\alpha+$ caroço}

Diferentes formas de potencial nuclear cluster + caroço foram usadas nos trabalhos que tratam das estruturas de cluster. Algumas formas se baseiam em variações dos potenciais do tipo Woods-Saxon, Gaussiano e "cosseno hiperbólico", enquanto outras tentam levar em conta a interação núcleon-núcleon de maneira mais explícita. No último exemplo mencionado, são usados os potenciais do tipo folding ou double folding [21] onde também são incluídas as funções de densidade de matéria do cluster e do caroço para a simulação da interação entre ambos. Em geral, os parâmetros dos potenciais são ajustados para a reprodução dos níveis de energia das bandas experimentais, mas há trabalhos na literatura que mostram potenciais cujos parâmetros são ajustados para a descrição de outros dados experimentais além dos níveis de energia, como as seções de choque de espalhamento elástico $\alpha+$ caroço $[4,5,14,22]$.

O potencial nuclear $\alpha+$ caroço usado neste trabalho foi desenvolvido por Buck, Merchant e Perez [3] e apresenta a forma 


$$
V_{N}(r)=-V_{0}\left\{\frac{b}{1+\exp \left(\frac{r-R}{a}\right)}+\frac{1-b}{\left[1+\exp \left(\frac{r-R}{3 a}\right)\right]^{3}}\right\},
$$

onde $V_{0}=220 \mathrm{MeV}, a=0.65 \mathrm{fm}, b=0.3$ e $R$ é o parâmetro radial ajustável para o núcleo a ser analisado. Foi verificado que esta forma de potencial é eficiente na descrição de dados espectroscópicos de diferentes núcleos onde a estrutura de cluster- $\alpha$ é esperada. Em adição à interação nuclear, é incluído o potencial coulombiano

$$
V_{C}(r)= \begin{cases}\left(\frac{e^{2}}{4 \pi \varepsilon_{0}}\right) \frac{Z_{\alpha} Z_{\mathrm{car}}}{2 R}\left(3-\frac{r^{2}}{R^{2}}\right) & \text { para } \quad r<R \\ \left(\frac{e^{2}}{4 \pi \varepsilon_{0}}\right) \frac{Z_{\alpha} Z_{\mathrm{car}}}{r} & \text { para } \quad r \geq R\end{cases}
$$

simulado pela interação entre uma partícula- $\alpha$ pontual e uma esfera uniformemente carregada de raio $R$, sendo $Z_{\alpha}$ e $Z_{\text {car }}$ respectivamente os números atômicos da partícula- $\alpha$ e do caroço, enquanto $R$ é o mesmo parâmetro usado em $V_{N}(r)$. O potencial total então será

$$
V(r)=V_{N}(r)+V_{C}(r) .
$$

A inclusão do termo associado à barreira centrífuga resulta no potencial efetivo

$$
V_{\mathrm{ef}}(r)=V(r)+\frac{L(L+1) \hbar^{2}}{2 \mu r^{2}}
$$

onde $\mu$ é a massa reduzida do sistema $\alpha+$ caroço.

Os potenciais $V_{N}(r)$ e $V(r)$ são mostrados graficamente na Figura 1.1 para o caso específico do sistema $\alpha+{ }^{90} \mathrm{Zr}$ com o valor de $R$ sugerido na Ref. [3]. De acordo com esta referência, os parâmetros $V_{0}, a$ e $b$ foram ajustados com a finalidade de reproduzir satisfatoriamente as energias de excitação experimentais das bandas de estado fundamental nos núcleos ${ }^{20} \mathrm{Ne},{ }^{44} \mathrm{Ti}$, ${ }^{94} \mathrm{Mo}$ e ${ }^{212} \mathrm{Po}$, além das meiasvidas de decaimento- $\alpha$ experimentais de vários núcleos pesados par-par, enquanto o parâmetro $R$ foi ajustado com referência na energia de separação- $\alpha$ específica de cada núcleo analisado naquele trabalho. A forma de $V_{N}(r)$ (uma combinação entre um termo de Woods-Saxon simples e outro de Woods-Saxon cúbico) foi escolhida pelo fato de ter sido a mais eficiente para a descrição simultânea dos dados experimentais citados anteriormente, além de ter apresentado, nos casos dos potenciais ajustados para os núcleos ${ }^{20} \mathrm{Ne}$ e ${ }^{44} \mathrm{Ti}$, uma similaridade com as partes reais dos potenciais ópticos determinados a partir do espalhamento elástico de partículas- $\alpha$ em alvos de ${ }^{16} \mathrm{O}$ e ${ }^{40} \mathrm{Ca}$, respectivamente (ver Figura 1.2). 
Neste trabalho, o potencial $V(r)$ em (1.5) é usado para a descrição de várias propriedades das estruturas $\alpha+$ caroço nos núcleos da região do Molibdênio, como é descrito nos capítulos seguintes. 


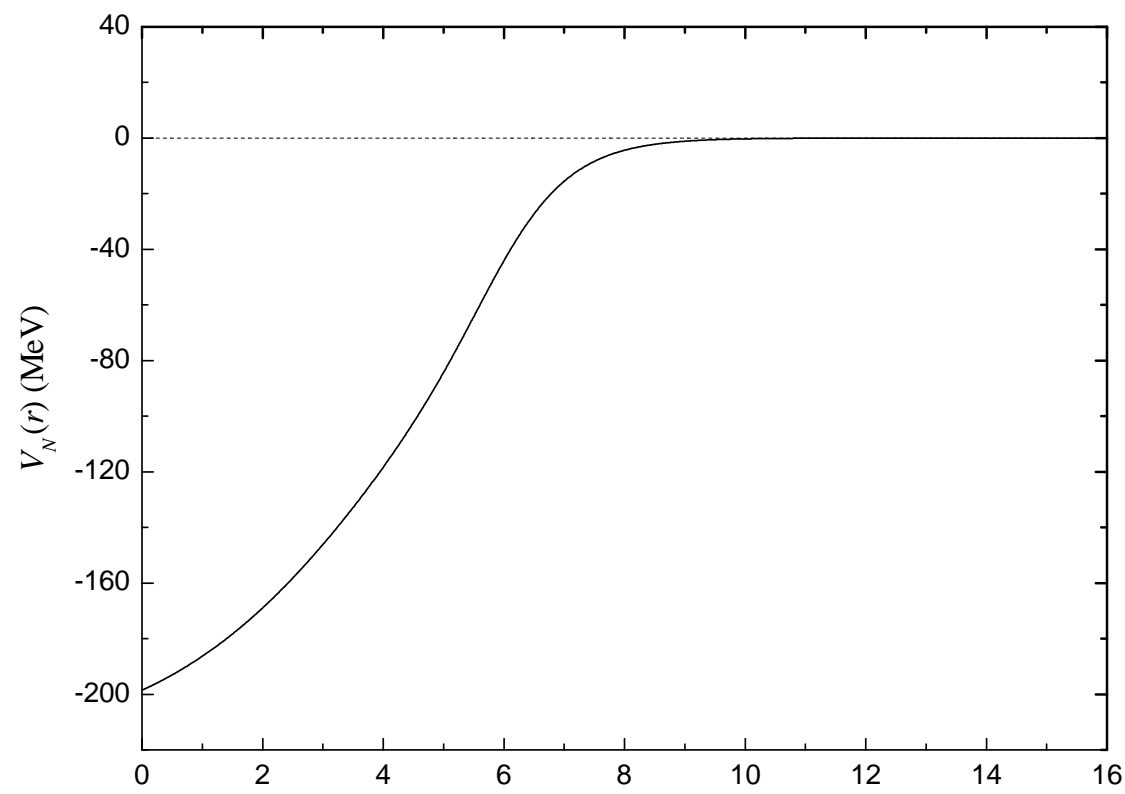

(a)

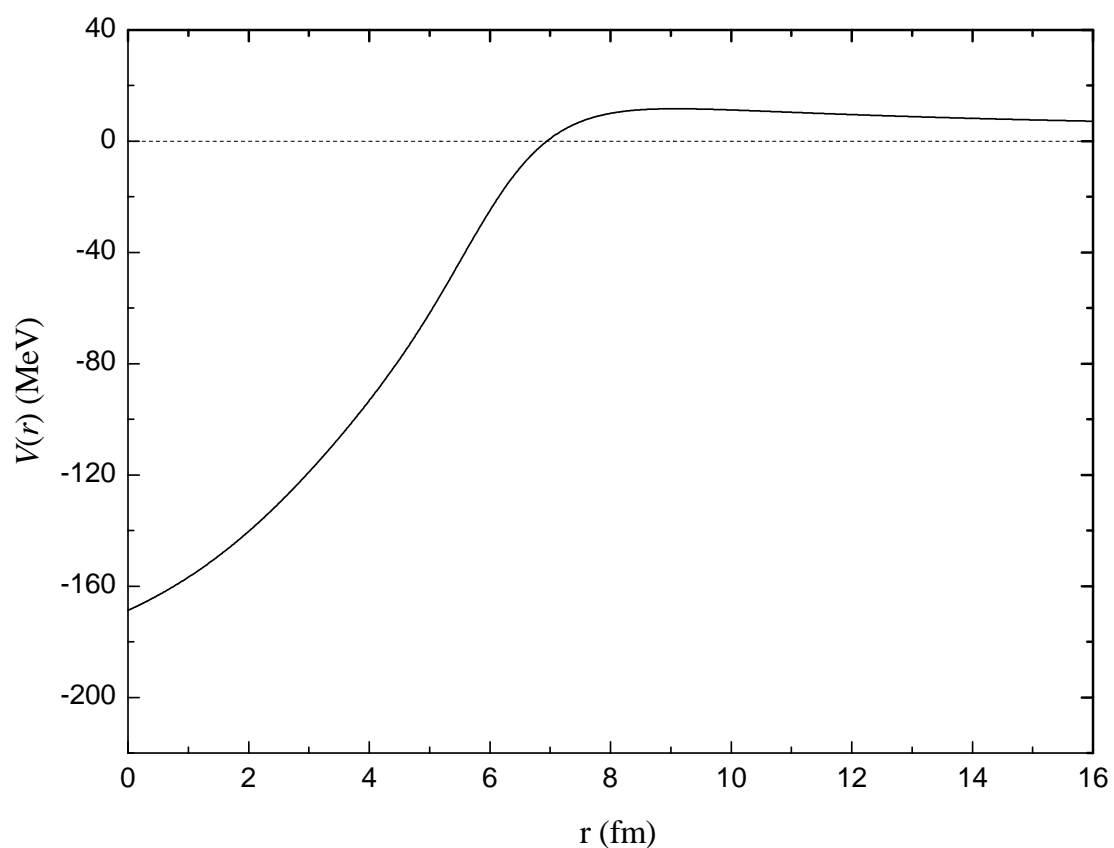

(b)

Figura 1.1: Potencial nuclear $V_{N}(r)$ (a) e potencial total $V(r)$ (b) referentes à interação $\alpha+{ }^{90} \mathrm{Zr}$. $\mathrm{O}$ valor aplicado ao parâmetro $R$ é aquele sugerido na Ref. [3] para o ${ }^{94} \mathrm{Mo}(5.793 \mathrm{fm})$. As linhas pontilhadas indicam o limiar $\alpha+{ }^{90} \mathrm{Zr}$. 

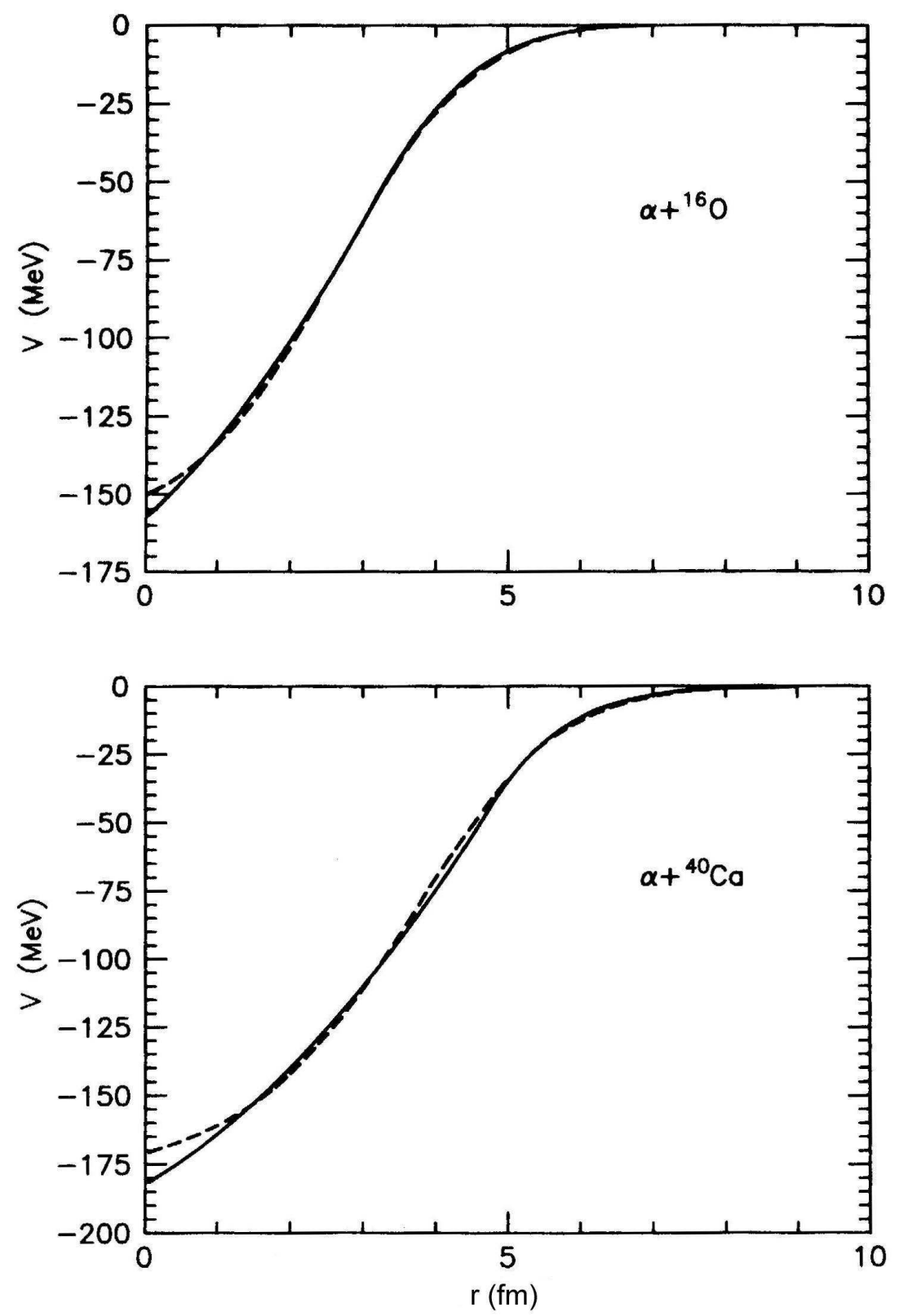

Figura 1.2: Comparação das partes reais dos potenciais ópticos determinados a partir do espalhamento- $\alpha$ elástico em alvos de ${ }^{16} \mathrm{O}$ e ${ }^{40} \mathrm{Ca}$ (linhas tracejadas) com os potenciais nucleares desenvolvidos na forma da eq. (1.3) para a descrição dos sistemas $\alpha+$ caroço nos núcleos ${ }^{20} \mathrm{Ne}$ e ${ }^{44} \mathrm{Ti}$, respectivamente (linhas contínuas). Esta figura foi extraída da Ref. [3]. 


\section{Capítulo 2}

\section{Processo de seleção dos núcleos estudados}

Neste capítulo, é descrito o critério usado para a seleção dos núcleos da região do Molibdênio que são preferenciais para o estudo da estrutura $\alpha+$ caroço. Primeiramente, o critério de seleção é aplicado em diferentes regiões de massa, visando confrontar os resultados obtidos com as escolhas feitas na literatura a respeito dos núcleos preferenciais para a aglomeração- $\alpha$. Em seguida, o critério de seleção é aplicado sistematicamente a vários conjuntos de isótopos e isóbaros par-par da região do Molibdênio. Os aspectos comuns observados entre os diferentes conjuntos de núcleos são discutidos.

\subsection{Critério de seleção dos núcleos com estrutura $\alpha+$ caroço}

O estudo da estrutura de cluster envolve inicialmente a escolha de núcleos onde esse tipo de estrutura é mais favorável. Diferentes critérios podem ser usados para tal escolha, podendo envolver princípios de algum modelo nuclear e dados experimentais de massa, meia-vida de decaimento ou energia de ligação. No caso da estrutura binária cluster-caroço, frequentemente é usado o critério onde o cluster e o caroço devem apresentar dupla camada fechada $[1,2,3,4]$ ou, ao menos, camada fechada de prótons (ou nêutrons) mais subcamada fechada de nêutrons (ou prótons). Outro critério se baseia na determinação do par cluster-caroço com a menor meia-vida de decaimento possível para o sistema [23] (este critério só pode ser aplicado em núcleos onde o valor-Q de separação cluster-caroço seja positivo). Um critério usado por Buck, Merchant e Perez [23, 24] para a escolha da estrutura cluster-caroço mais favorável para um certo núcleo de número atômico $Z$ e número de massa $A$ é determinar o par cluster-caroço que resulta no valor máximo para a 
grandeza

$$
\begin{aligned}
& D\left(Z_{1}, A_{1}, Z_{2}, A_{2}\right)= \\
& \quad\left[B_{A}\left(Z_{1}, A_{1}\right)-B_{M}\left(Z_{1}, A_{1}\right)\right]+\left[B_{A}\left(Z_{2}, A_{2}\right)-B_{M}\left(Z_{2}, A_{2}\right)\right],
\end{aligned}
$$

onde $B_{A}\left(Z_{i}, A_{i}\right)$ é a energia de ligação real (aquela resultante de dados experimentais) do cluster (ou caroço) de número atômico $Z_{i}$ e número de massa $A_{i}$ e $B_{M}\left(Z_{i}, A_{i}\right)$ é a energia de ligação calculada pelo modelo de gota líquida para o mesmo cluster (ou caroço), sendo que o cluster é caracterizado por $\left(Z_{1}, A_{1}\right)$ e o caroço é caracterizado por $\left(Z_{2}, A_{2}\right)=\left(Z-Z_{1}, A-A_{1}\right)$.

Todos os critérios mencionados anteriormente podem ser aplicados ou adaptados para o caso específico da estrutura $\alpha+$ caroço. Neste trabalho, é buscado um critério simples para a determinação do núcleo mais favorável (ou núcleos mais favoráveis) para a aglomeração- $\alpha$ dentro de um conjunto especificado, somente com o uso de dados experimentais de energia de ligação (ou massa). Para isso, deseja-se encontrar o maior acréscimo (ou menor decréscimo) de energia de ligação por núcleon devido à decomposição $\alpha$-caroço nos núcleos do conjunto. O cálculo deste valor inicia-se com a definição das grandezas

$$
b_{T}=\frac{B_{T}}{A_{T}} \quad \overline{b_{\alpha-\mathrm{car}}}=\frac{B_{\alpha}+B_{\mathrm{car}}}{A_{T}},
$$

onde $B_{T}, B_{\alpha}$ e $B_{\text {car }}$ são as energias de ligação do núcleo total, do cluster- $\alpha$ e do caroço, respectivamente, e $A_{T}$ é o número de massa do núcleo total. Com isso, $b_{T}$ é a energia de ligação por núcleon do núcleo total e $\overline{b_{\alpha-\text { car }}}$ é a energia de ligação média por núcleon do par $\alpha$-caroço. No caso de $\overline{b_{\alpha-\text { car }}}$, considera-se apenas as energias de ligação internas do cluster- $\alpha$ e do caroço, desconsiderando, portanto, energias da interação entre núcleons do cluster- $\alpha$ e os núcleons do caroço. O cálculo de $\overline{b_{\alpha-\text { car }}}$ é equivalente a determinar a energia de ligação por núcleon do sistema $\alpha+$ caroço quando há a possibilidade de separação dos dois subnúcleos, isto é, quando o sistema encontra-se no limiar de energia para a separação- $\alpha$. Para demonstrarmos isso, consideremos a energia da massa do núcleo total

$$
E_{T}=M_{T} c^{2}=\left(Z_{T} m_{p}+N_{T} m_{n}\right) c^{2}-B_{T},
$$

onde $M_{T}$ é a massa do núcleo total, $Z_{T}$ e $N_{T}$ são o número de prótons e o número de nêutrons do núcleo total, respectivamente, e $m_{p}$ e $m_{n}$ são as massas do próton e do nêutron, respectivamente. Em termos das massas do cluster- $\alpha\left(M_{\alpha}\right)$ e do caroço $\left(M_{\text {car }}\right)$, temos 


$$
\begin{aligned}
E_{T}= & M_{\alpha} c^{2}+M_{\mathrm{car}} c^{2}-E_{\alpha-\mathrm{car}}= \\
& \left(Z_{\alpha} m_{p}+N_{\alpha} m_{n}+Z_{\mathrm{car}} m_{p}+N_{\mathrm{car}} m_{n}\right) c^{2}-B_{\alpha}-B_{\mathrm{car}}-E_{\alpha-\mathrm{car}}
\end{aligned}
$$

onde $E_{\alpha \text {-car }}$ é a energia de separação $\alpha$-caroço, $Z_{\alpha}$ e $Z_{\text {car }}$ são os números de prótons do cluster- $\alpha$ e do caroço, respectivamente, e $N_{\alpha}$ e $N_{\text {car }}$ são os números de nêutrons do cluster- $\alpha$ e do caroço, respectivamente. Confrontando (2.3) e (2.4) e lembrando que $Z_{T}=Z_{\alpha}+Z_{\text {car }}$ e $N_{T}=N_{\alpha}+N_{\text {car }}$, temos:

$$
B_{T}=B_{\alpha}+B_{\text {car }}+E_{\alpha-\text { car }} .
$$

Se o núcleo total é excitado numa energia $E_{\alpha \text {-car }}$ (no limiar de separação $\alpha$-caroço), a energia resultante $E_{T}^{\mathrm{lim}}$ do sistema torna-se

$$
\begin{aligned}
E_{T}^{\lim }= & E_{T}+E_{\alpha-\mathrm{car}}= \\
& \left(Z_{T} m_{p}+N_{T} m_{n}\right) c^{2}-B_{T}+E_{\alpha-\mathrm{car}}=\left(Z_{T} m_{p}+N_{T} m_{n}\right) c^{2}-B_{\lim },
\end{aligned}
$$

onde $B_{\lim }=B_{\alpha}+B_{\text {car }}$ é interpretado como a energia de ligação efetiva do sistema quando este está excitado numa energia $E_{\alpha-\text { car }}$. Assim, calculando a energia de ligação por núcleon do sistema excitado, teremos:

$$
\frac{B_{\lim }}{A_{T}}=\frac{B_{\alpha}+B_{\mathrm{car}}}{A_{T}}=\overline{b_{\alpha-\mathrm{car}}} .
$$

A comparação entre $b_{T}$ e $\overline{b_{\alpha-\text { car }}}$ permite mostrar se há uma fraca ou forte tendência do núcleo total para chegar ao estado de separação do par $\alpha$-caroço. Temos então

$$
\Delta b=\overline{b_{\alpha-\mathrm{car}}}-b_{T}=\frac{B_{\alpha}+B_{\mathrm{car}}-B_{T}}{A_{T}}=\frac{Q_{\alpha}}{A_{T}},
$$

onde $Q_{\alpha}$ é o valor-Q de separação- $\alpha$ do núcleo total. Desta forma, a grandeza $Q_{\alpha} / A_{T}$ determina o aumento ou diminuição da energia de ligação por núcleon do sistema devido à decomposição $\alpha$-caroço. A comparação dos valores de $Q_{\alpha} / A_{T}$ para diferentes núcleos indica qual é o núcleo deste conjunto com a condição mais favorável de aglomeração- $\alpha$, sendo o valor máximo de $Q_{\alpha} / A_{T}$ aquele que identifica o núcleo mais favorável. O valor máximo de $Q_{\alpha} / A_{T}$ pode ser positivo ou negativo, representando, respectivamente, o maior acréscimo ou o menor decréscimo de energia de ligação por núcleon dentre os núcleos comparados. 


\subsection{Avaliação preliminar do critério de $Q_{\alpha} / A_{T}$}

Visando testar o critério de comparação de $Q_{\alpha} / A_{T}$, foram escolhidos alguns conjuntos de isótopos e isóbaros que incluem os núcleos ${ }^{20} \mathrm{Ne},{ }^{44} \mathrm{Ti},{ }^{94} \mathrm{Mo} \mathrm{e}{ }^{212} \mathrm{Po}$. Estes quatro núcleos, em suas respectivas regiões, são tratados na literatura como preferenciais na investigação da estrutura $\alpha+$ caroço, pois todos apresentam caroços de dupla camada fechada (no caso do ${ }^{94} \mathrm{Mo}$, o caroço de ${ }^{90} \mathrm{Zr}$ se comporta similarmente a um núcleo de dupla camada fechada). É interessante verificar se há correspondência entre os resultados do critério de $Q_{\alpha} / A_{T}$ e as escolhas de núcleos com caroços definidos pelos efeitos de camada fechada.

As comparações dos diferentes conjuntos de isótopos e isóbaros são mostradas nas Figuras de 2.1 a 2.8. Os valores de $Q_{\alpha} / A_{T}$ foram calculados através de tabelas com dados experimentais de energia de ligação [18]. Os núcleos par-ímpar e ímparímpar não são considerados nas comparações, pois tais núcleos apresentam em geral uma configuração do sistema $\alpha+$ caroço onde o caroço possui spin não nulo em seu estado fundamental. A presença de um caroço com spin não nulo provoca o surgimento de forças não-centrais na interação $\alpha$-caroço e acoplamentos de momentos angulares que são evitados nesta etapa do trabalho, considerando-se que há várias complicações na resolução do sistema para o referido caso. Além disso, para muitos núcleos par-ímpar e ímpar-ímpar, não há coincidência entre o spin total $J$ do estado de mais baixa energia do sistema $\alpha$ + caroço e o spin do estado fundamental do núcleo total, de forma que, nestes casos, não é possível descrever o estado fundamental do núcleo total como o primeiro nível de energia do sistema $\alpha+$ caroço. Visando aplicar o critério de $Q_{\alpha} / A_{T}$ somente a núcleos que podem ter os seus estados fundamentais associados à estrutura simples $\alpha+$ (caroço de spin nulo), nos restringimos a comparar apenas os núcleos par-par.

Os gráficos presentes nas Figuras de 2.1 a 2.8 mostram máximos absolutos e máximos locais de $Q_{\alpha} / A_{T}$ nos conjuntos de isótopos e isóbaros. Na comparação dos isótopos de Ne (Figura 2.1), temos um máximo absoluto de $Q_{\alpha} / A_{T}$ em $A_{T}=20$ e outro máximo local em $A_{T}=28$. Os dois valores de $A_{T}$ mencionados correspondem aos núcleos ${ }^{20} \mathrm{Ne}$ e ${ }^{28} \mathrm{Ne}$, os quais possuem as estruturas $\alpha+{ }^{16} \mathrm{O}$ e $\alpha+{ }^{24} \mathrm{O}$, respectivamente, onde o caroço de ${ }^{16} \mathrm{O}$ apresenta dupla camada fechada ( 8 prótons e 8 nêutrons) e o ${ }^{24} \mathrm{O}$ apresenta camada fechada de prótons e a subcamada $2 s_{1 / 2}$ completa para nêutrons (as discussões sobre o preenchimento de camadas e subcamadas de prótons e nêutrons se baseiam nos diagramas de energia de partícula simples do modelo de camadas [25, 26, 27, 28, 29]). Na comparação dos isóbaros de $A_{T}=20$ (Figura 2.2), temos um máximo absoluto de $Q_{\alpha} / A_{T}$ correspondendo ao ${ }^{20} \mathrm{Ne}$. Mostra-se claramente que o ${ }^{20} \mathrm{Ne}$ é o núcleo preferencial para a aglomeração- $\alpha$ quando é comparado aos seus isótopos e isóbaros simultaneamente. 
$Z_{T}=10$

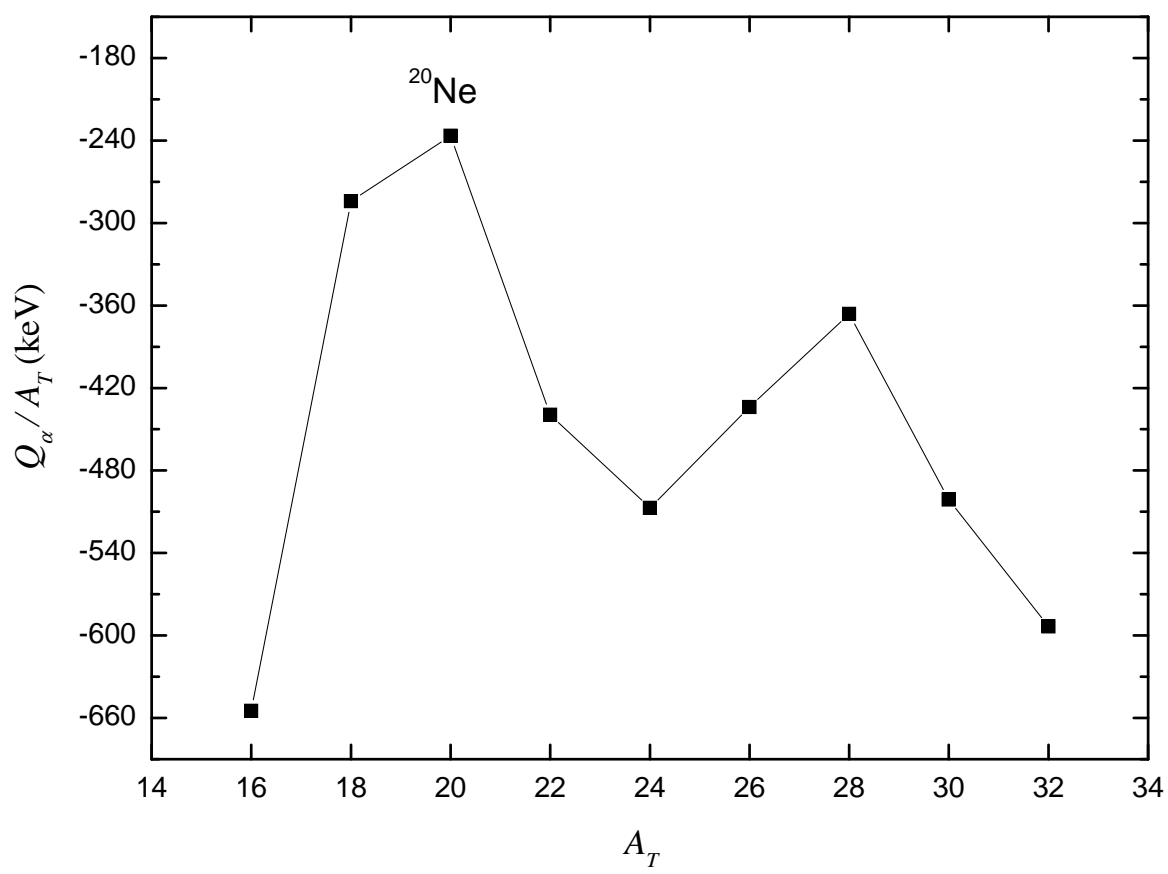

Figura 2.1: Comparação entre os valores de $Q_{\alpha} / A_{T}$ para os isótopos par-par de $\mathrm{Ne}\left(Z_{T}=10\right)$. É indicado o máximo absoluto de $Q_{\alpha} / A_{T}$ que corresponde ao ${ }^{20} \mathrm{Ne}$.

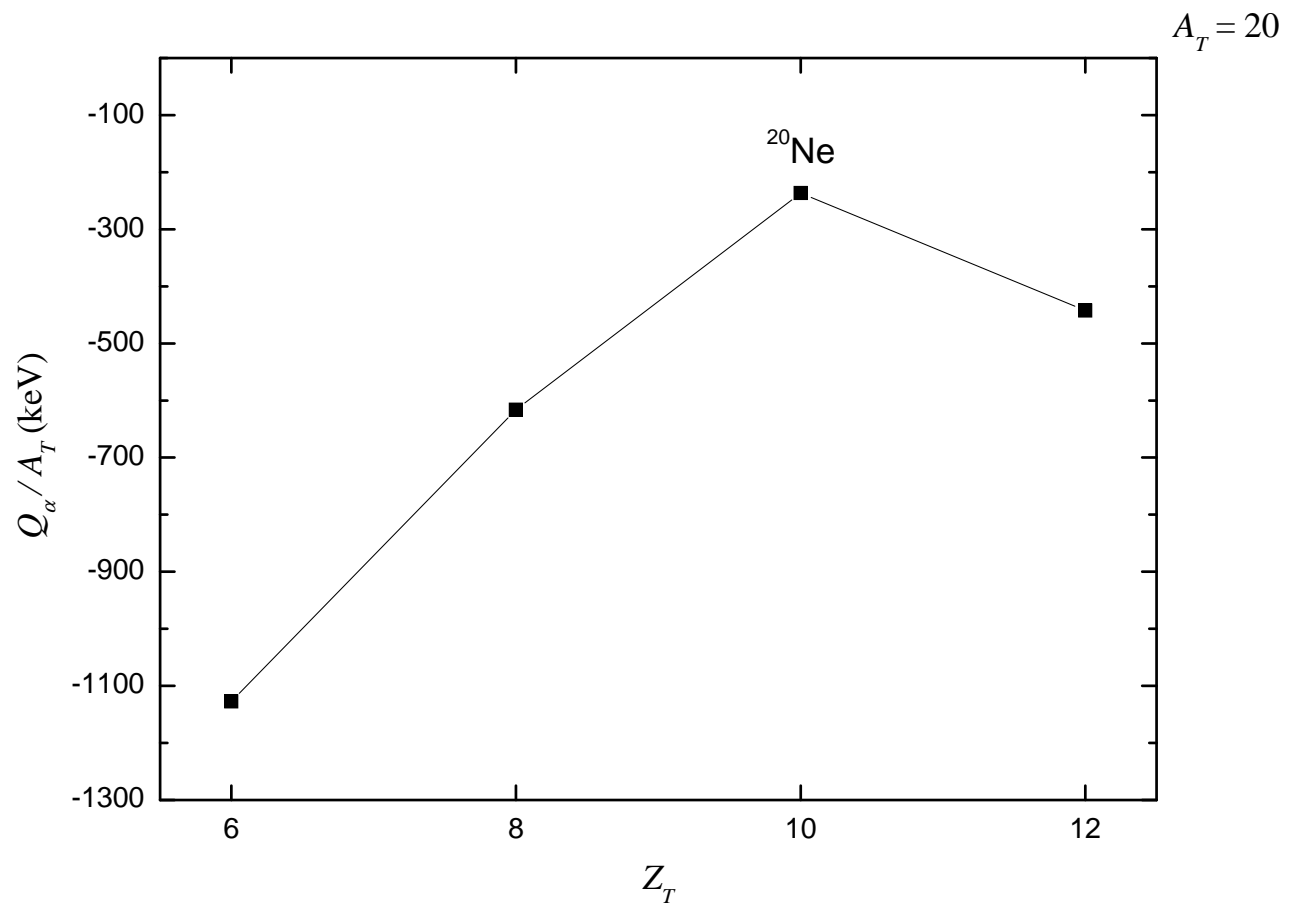

Figura 2.2: Comparação entre os valores de $Q_{\alpha} / A_{T}$ para os isóbaros par-par de $A_{T}=20$. É indicado o máximo absoluto de $Q_{\alpha} / A_{T}$ que corresponde ao ${ }^{20} \mathrm{Ne}$. 


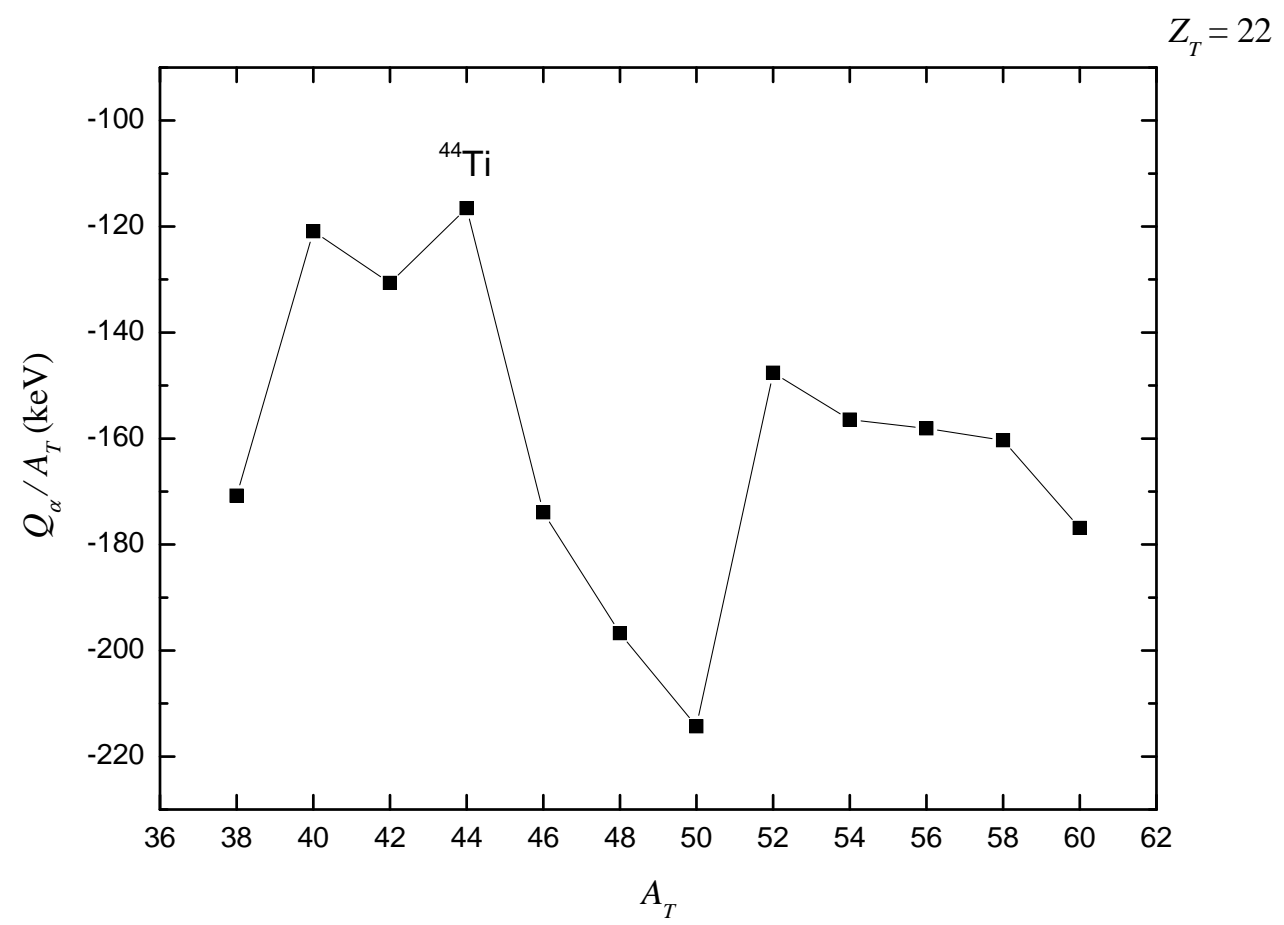

Figura 2.3: Comparação entre os valores de $Q_{\alpha} / A_{T}$ para os isótopos par-par de Ti $\left(Z_{T}=22\right)$. É indicado o máximo absoluto de $Q_{\alpha} / A_{T}$ que corresponde ao ${ }^{44} \mathrm{Ti}$.

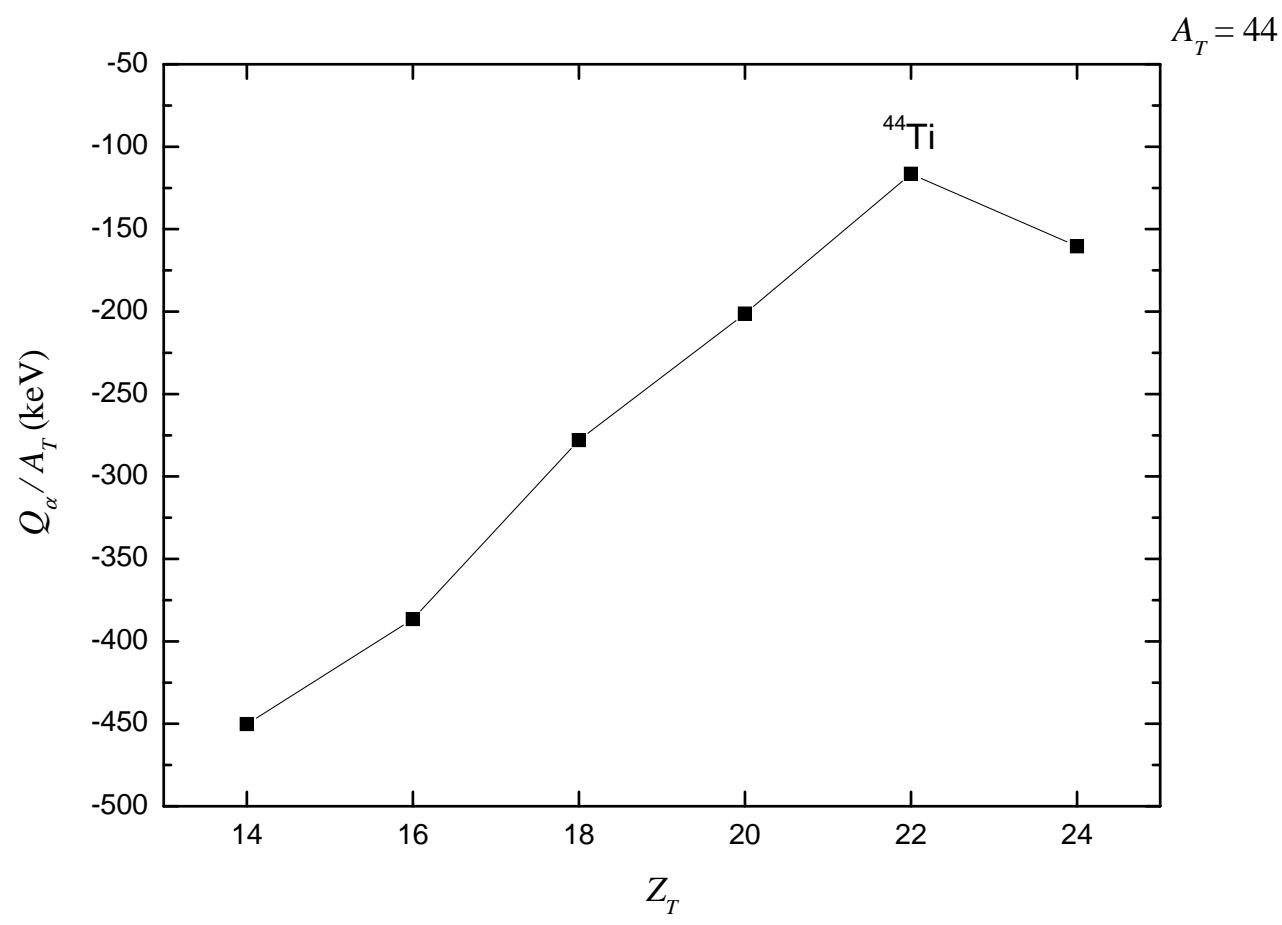

Figura 2.4: Comparação entre os valores de $Q_{\alpha} / A_{T}$ para os isóbaros par-par de $A_{T}=44$. É indicado o máximo absoluto de $Q_{\alpha} / A_{T}$ que corresponde ao ${ }^{44} \mathrm{Ti}$. 


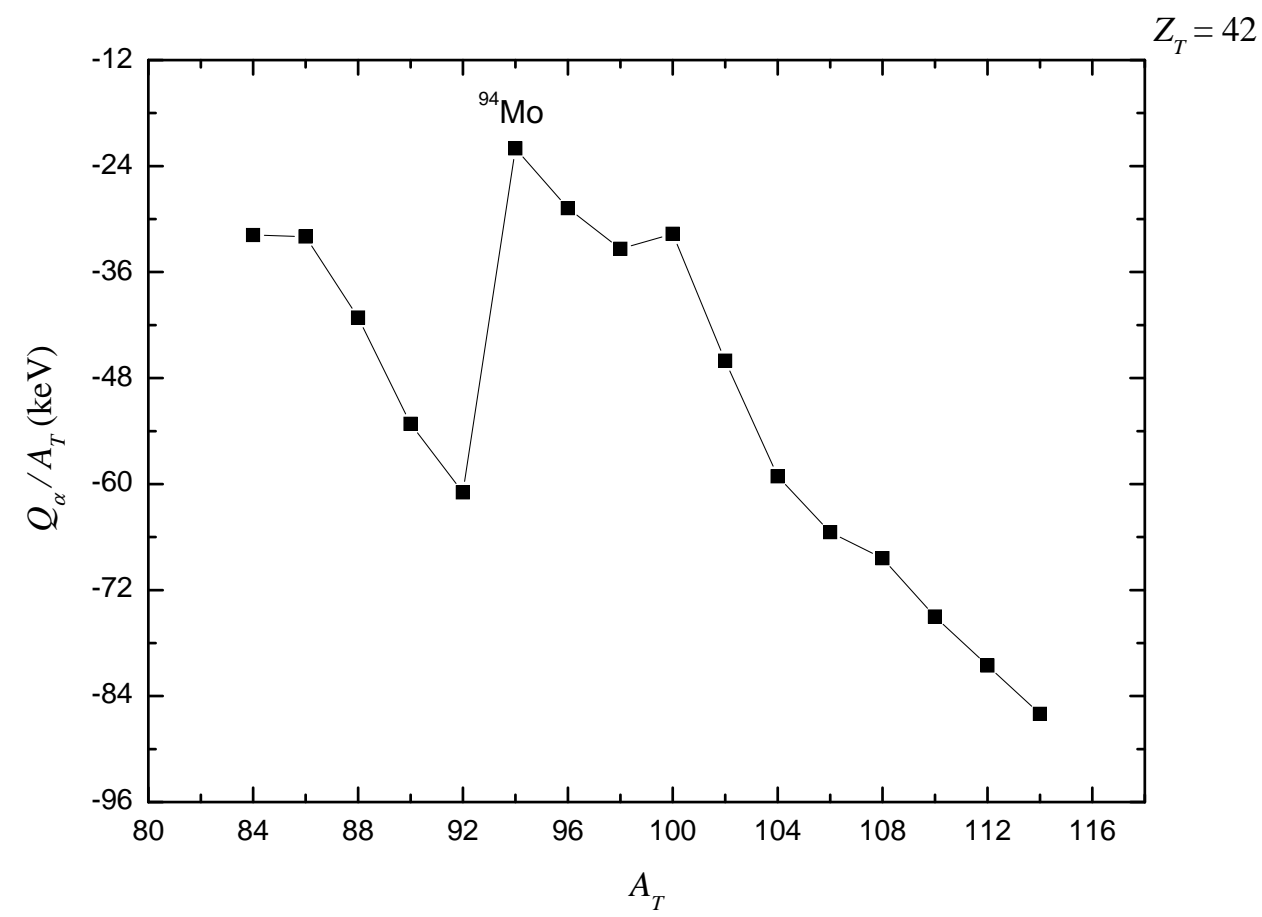

Figura 2.5: Comparação entre os valores de $Q_{\alpha} / A_{T}$ para os isótopos par-par de Mo $\left(Z_{T}=42\right)$. É indicado o máximo absoluto de $Q_{\alpha} / A_{T}$ que corresponde ao ${ }^{94} \mathrm{Mo}$.

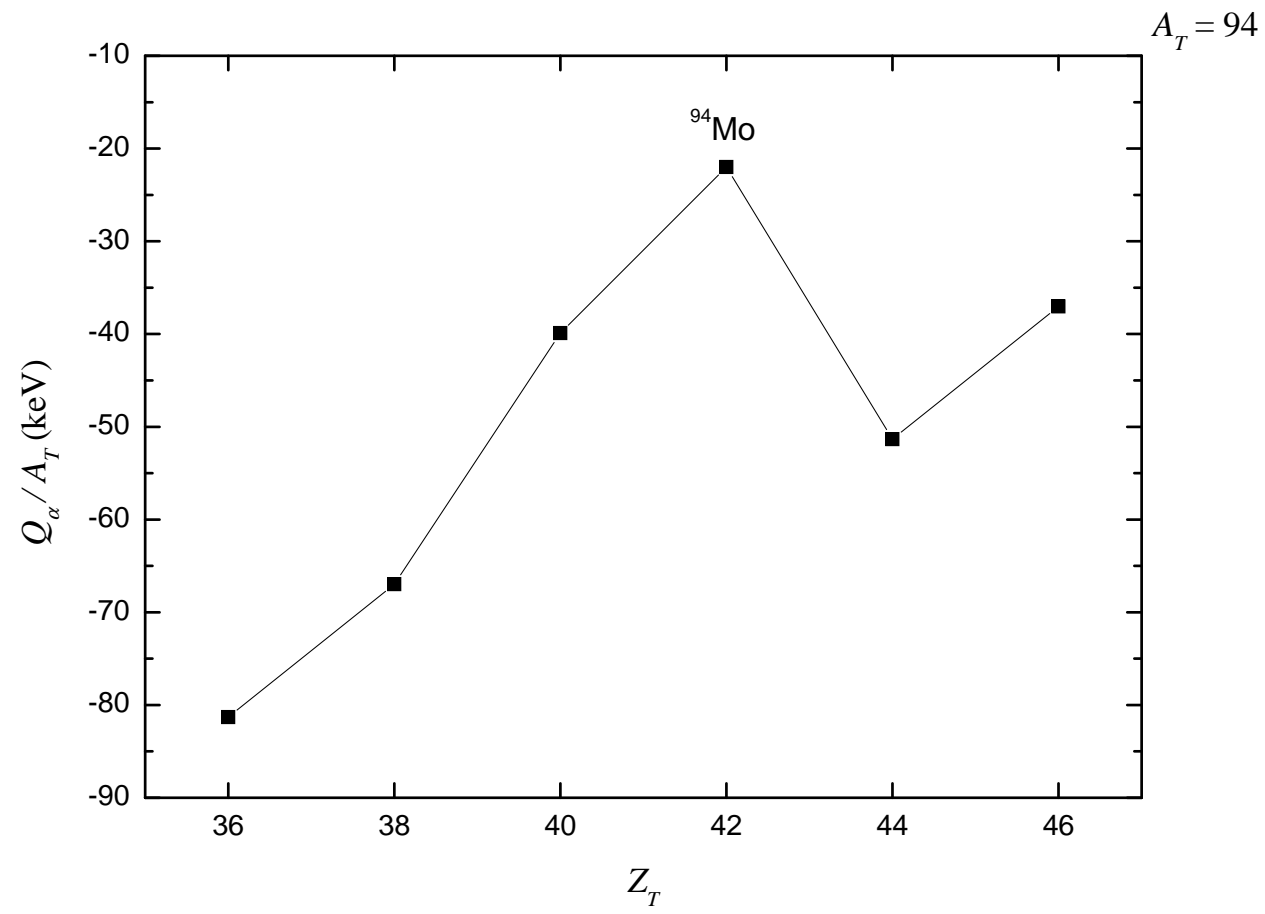

Figura 2.6: Comparação entre os valores de $Q_{\alpha} / A_{T}$ para os isóbaros par-par de $A_{T}=94$. É indicado o máximo absoluto de $Q_{\alpha} / A_{T}$ que corresponde ao ${ }^{94} \mathrm{Mo}$. 


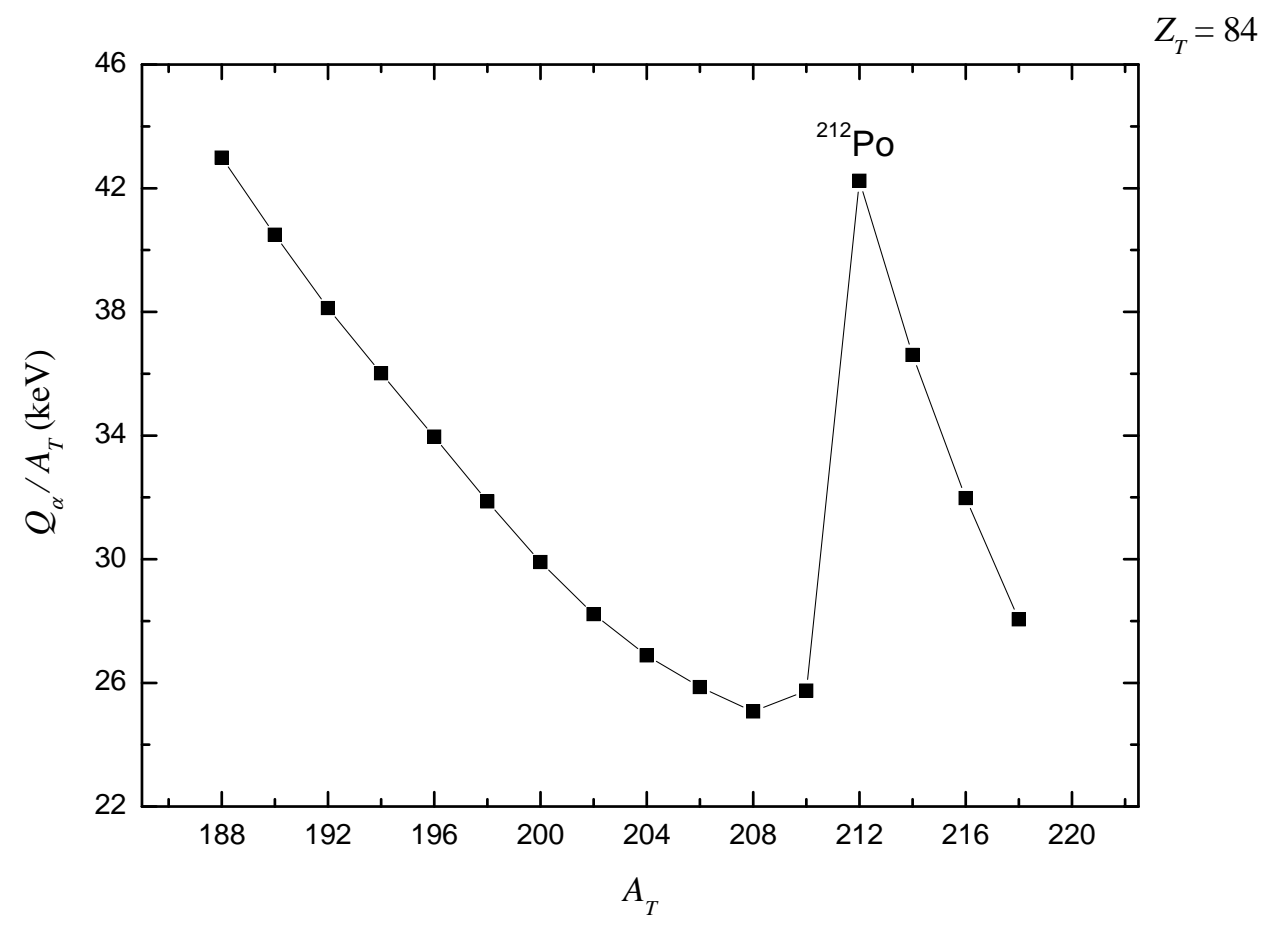

Figura 2.7: Comparação entre os valores de $Q_{\alpha} / A_{T}$ para os isótopos par-par de Po $\left(Z_{T}=84\right)$. É indicado o máximo local de $Q_{\alpha} / A_{T}$ que corresponde ao ${ }^{212} \mathrm{Po}$.

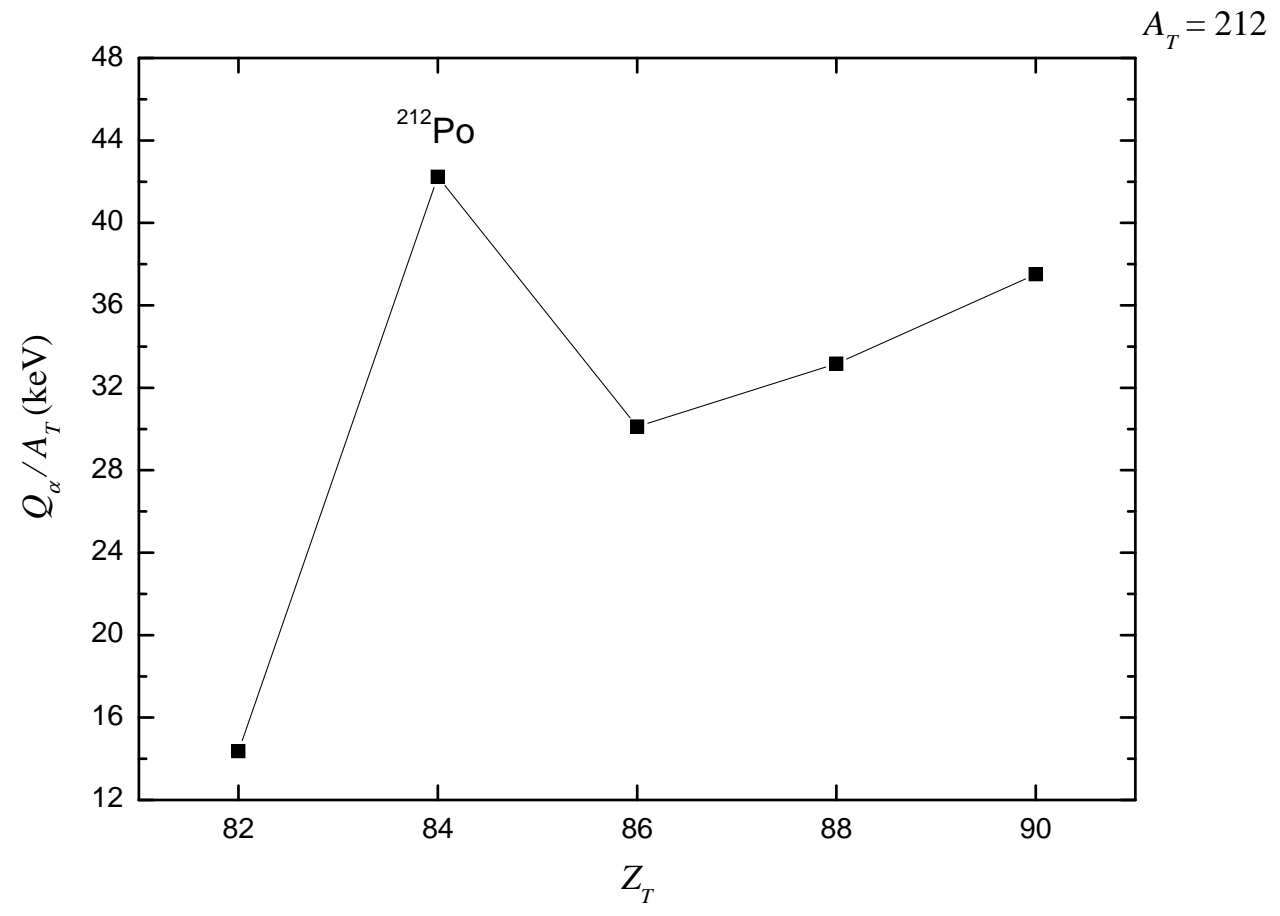

Figura 2.8: Comparação entre os valores de $Q_{\alpha} / A_{T}$ para os isóbaros par-par de $A_{T}=212$. É indicado o máximo absoluto de $Q_{\alpha} / A_{T}$ que corresponde ao ${ }^{212} \mathrm{Po}$. 
Na comparação dos isótopos de Ti (Figura 2.3), temos um máximo absoluto de $Q_{\alpha} / A_{T}$ em $A_{T}=44$ e outros dois máximos locais em $A_{T}=40$ e $A_{T}=52$. Os três valores de $A_{T}$ mencionados correspondem aos núcleos ${ }^{44} \mathrm{Ti},{ }^{40} \mathrm{Ti}$ e ${ }^{52} \mathrm{Ti}$, os quais possuem as estruturas $\alpha+{ }^{40} \mathrm{Ca}, \alpha+{ }^{36} \mathrm{Ca}$ e $\alpha+{ }^{48} \mathrm{Ca}$, respectivamente. Nos sistemas mencionados, o caroço de ${ }^{40} \mathrm{Ca}$ apresenta dupla camada fechada (20 prótons e 20 nêutrons), o ${ }^{36} \mathrm{Ca}$ apresenta camada fechada de prótons e a subcamada $2 s_{1 / 2}$ completa para nêutrons, e o ${ }^{48} \mathrm{Ca}$ apresenta dupla camada fechada (20 prótons e 28 nêutrons). Na comparação dos isóbaros de $A_{T}=44$ (Figura 2.4), temos um máximo absoluto de $Q_{\alpha} / A_{T}$ correspondendo ao ${ }^{44} \mathrm{Ti}$. De forma análoga ao caso do ${ }^{20} \mathrm{Ne}$, mostra-se que o ${ }^{44} \mathrm{Ti}$ é o núcleo preferencial para a aglomeração- $\alpha$ quando é comparado aos seus isótopos e isóbaros simultaneamente.

Na comparação dos isótopos de Mo (Figura 2.5), temos um máximo absoluto de $Q_{\alpha} / A_{T}$ em $A_{T}=94$ e outros máximos locais em $A_{T}=84$ e $A_{T}=100$. Os três valores de $A_{T}$ mencionados correspondem aos núcleos ${ }^{94} \mathrm{Mo},{ }^{84} \mathrm{Mo} \mathrm{e}{ }^{100} \mathrm{Mo}$, os quais possuem as estruturas $\alpha+{ }^{90} \mathrm{Zr}, \alpha+{ }^{80} \mathrm{Zr}$ e $\alpha+{ }^{96} \mathrm{Zr}$, respectivamente. Nos sistemas mencionados, o caroço de ${ }^{90} \mathrm{Zr}$ apresenta a subcamada $2 p_{1 / 2}$ completa para prótons e camada fechada de nêutrons (50 nêutrons), o ${ }^{80} \mathrm{Zr}$ apresenta a subcamada $2 p_{1 / 2}$ completa para prótons e nêutrons, e o ${ }^{96} \mathrm{Zr}$ apresenta a subcamada $2 p_{1 / 2}$ completa para prótons e a subcamada $2 d_{5 / 2}$ completa para nêutrons (considera-se que a subcamada $2 d_{5 / 2}$ encontra-se abaixo da subcamada $1 g_{7 / 2}$, de acordo com certos diagramas de energia de partícula simples na literatura $[27,28]$ ). Na comparação dos isóbaros de $A_{T}=94$ (Figura 2.6), temos um máximo absoluto de $Q_{\alpha} / A_{T}$ correspondendo ao ${ }^{94} \mathrm{Mo}$. De forma análoga ao casos do ${ }^{20} \mathrm{Ne}$ e ${ }^{44} \mathrm{Ti}$, mostra-se que o ${ }^{94}$ Mo é o núcleo preferencial para a aglomeração- $\alpha$ quando é comparado aos seus isótopos e isóbaros simultaneamente.

Fazendo o mesma comparação para os isótopos de Po (Figura 2.7), temos que o valor máximo absoluto de $Q_{\alpha} / A_{T}$ está em $A_{T}=188$, mas há um máximo local de grande evidência em $A_{T}=212$. Os dois valores de $A_{T}$ mencionados correspondem aos núcleos ${ }^{188} \mathrm{Po}$ e ${ }^{212} \mathrm{Po}$, os quais possuem as estruturas $\alpha+{ }^{184} \mathrm{~Pb}$ e $\alpha+{ }^{208} \mathrm{~Pb}$, respectivamente. Nos sistemas mencionados, o caroço de ${ }^{184} \mathrm{~Pb}$ apresenta camada fechada de prótons (82 prótons) e subcamada incompleta para nêutrons (na camada $3 p 2 f 1 h$, a ordem dos níveis é mostrada de diferentes formas nos diagramas de energia da literatura, mas os 102 nêutrons constituintes do ${ }^{184} \mathrm{~Pb}$ não preenchem completamente uma subcamada em todos os diagramas pesquisados), enquanto o ${ }^{208} \mathrm{~Pb}$ apresenta dupla camada fechada (82 prótons e 126 nêutrons). Na comparação dos isóbaros de $A_{T}=212$ (Figura 2.8), temos um máximo absoluto de $Q_{\alpha} / A_{T}$ correspondendo ao ${ }^{212} \mathrm{Po}$. Assim, avaliando simultaneamente os isótopos de Po e os isóbaros de $A_{T}=212$, conclui-se que o ${ }^{212}$ Po é o núcleo preferencial para a aglomeração- $\alpha$.

Após as comparações entre os núcleos dos diferentes conjuntos de isótopos e 
isóbaros, nota-se que todos os máximos absolutos e locais de $Q_{\alpha} / A_{T}$ estão associados a núcleos cujas estruturas $\alpha+$ caroço são constituídas de caroços de camada fechada ou subcamada completa de prótons e/ou nêutrons. Portanto, o critério de comparação de $Q_{\alpha} / A_{T}$ demonstra claramente o efeito do modelo de camadas na determinação da estrutura $\alpha+$ caroço mais favorável. Os caroços nucleares são mais estáveis (isto é, apresentam maior energia de ligação) quando possuem camada(s) fechada(s) ou subcamada(s) completa(s), resultando em núcleos totais com valores mais altos de $Q_{\alpha} / A_{T}$ comparativamente aos isótopos e isóbaros vizinhos. Especificamente no caso dos isótopos de Po, não se pode afirmar que o núcleo ${ }^{188} \mathrm{Po}$ apresenta o valor máximo absoluto de $Q_{\alpha} / A_{T}$ por efeitos de camada fechada, considerando a subcamada incompleta de nêutrons do caroço de ${ }^{184} \mathrm{~Pb}$. É importante destacar que outras propriedades nucleares exercem influência sobre os valores de $Q_{\alpha} / A_{T}$ (como efeitos de gota líquida, por exemplo), podendo resultar num alto valor de $Q_{\alpha} / A_{T}$ para um certo núcleo sem a contribuição dos efeitos do modelo de camadas.

Uma avaliação geral dos gráficos de $Q_{\alpha} / A_{T}$ desta Seção nos confirma que os núcleos ${ }^{20} \mathrm{Ne},{ }^{44} \mathrm{Ti},{ }^{94} \mathrm{Mo} \mathrm{e}{ }^{212} \mathrm{Po}$ são mais favoráveis para a aglomeração- $\alpha$ do que seus respectivos isótopos e isóbaros, corroborando as citações na literatura de que tais núcleos são os mais apropriados para uma primeira investigação da estrutura de cluster- $\alpha$ em suas respectivas regiões de massa.

\subsection{Seleção dos núcleos preferenciais para a aglomeração- $\alpha$ na região do Molibdênio}

Após a avaliação da eficiência do critério de $Q_{\alpha} / A_{T}$ para diferentes regiões de massa, o mesmo critério foi aplicado para a seleção dos núcleos com a estrutura $\alpha+$ caroço mais favorável na região do Mo. Como esta região inclui uma grande faixa de números atômicos e números de massa, foi estabelecido que este trabalho estaria restrito ao estudo de alguns conjuntos de isótopos próximos de $Z=42$, que é o número atômico do ${ }^{94}$ Mo. Abaixo de $Z=42$, foram escolhidos os isótopos de $\mathrm{Sr}$ e $\mathrm{Zr}$ e, acima de $Z=42$, os isótopos de $\mathrm{Ru}$ e $\mathrm{Pd}$. Pelos mesmos motivos descritos na seção anterior, excluímos das comparações de $Q_{\alpha} / A_{T}$ os núcleos com número ímpar de prótons e/ou nêutrons.

O procedimento adotado para a comparação entre os núcleos da região do Mo é próximo daquele descrito na Seção 2.2. O critério de comparação de $Q_{\alpha} / A_{T}$ é aplicado inicialmente a um conjunto de isótopos e, se algum núcleo do conjunto corresponde a um máximo absoluto de $Q_{\alpha} / A_{T}$ ou a um máximo local de $Q_{\alpha} / A_{T}$ de maior importância, o referido núcleo também será comparado aos seus isóbaros. É desejável que este núcleo também corresponda a um máximo absoluto de $Q_{\alpha} / A_{T}$ 
ou a um máximo local de $Q_{\alpha} / A_{T}$ entre os isóbaros, pois assim é mostrado que o mesmo é um núcleo preferencial para a aglomeração- $\alpha$ em relação aos seus isótopos e isóbaros simultaneamente.

As comparações dos diferentes conjuntos de isótopos e isóbaros desta região encontram-se nas Figuras de 2.9 a 2.16. Os valores de $Q_{\alpha} / A_{T}$ foram calculados através de tabelas com dados experimentais de energia de ligação [18]. Discutindo inicialmente a comparação dos isótopos de Sr (Figura 2.9), temos um valor máximo absoluto de $Q_{\alpha} / A_{T}$ em $A_{T}=74$ e outro máximo local em $A_{T}=90$. Os dois valores de $A_{T}$ mencionados correspondem aos núcleos ${ }^{74} \mathrm{Sr}$ e ${ }^{90} \mathrm{Sr}$, os quais possuem as estruturas $\alpha+{ }^{70} \mathrm{Kr}$ e $\alpha+{ }^{86} \mathrm{Kr}$, respectivamente, onde o caroço de ${ }^{70} \mathrm{Kr}$ apresenta a subcamada $1 f_{5 / 2}$ incompleta para prótons e nêutrons e o ${ }^{86} \mathrm{Kr}$ apresenta a subcamada $1 f_{5 / 2}$ incompleta para prótons e camada fechada de nêutrons (50 nêutrons). Apesar do ${ }^{74} \mathrm{Sr}$ apresentar o valor máximo absoluto de $Q_{\alpha} / A_{T}$, verifica-se na literatura que este é um núcleo de alta instabilidade (conforme a Ref. [30], o ${ }^{74} \mathrm{Sr}$ possui meia-vida $>1.2 \mu \mathrm{s}$ ) e o seu único estado conhecido experimentalmente é o próprio estado fundamental. Portanto, torna-se inviável o estudo da estrutura de cluster- $\alpha$ do ${ }^{74} \mathrm{Sr}$ e a análise dos isóbaros de $A_{T}=74$. Com isto, ${ }^{90} \mathrm{Sr}$ foi escolhido para ser comparado aos seus isóbaros de $A_{T}=90$ (Figura 2.10), sendo mostrada a presença de um máximo local de $Q_{\alpha} / A_{T}$ correspondendo ao mesmo núcleo. Mostra-se então que o ${ }^{90} \mathrm{Sr}$ é o núcleo preferencial para a aglomeração- $\alpha$ quando é comparado aos seus isótopos vizinhos e isóbaros vizinhos simultaneamente e, por este fato, foi considerado o isótopo de Sr mais apropriado para uma análise da estrutura de cluster- $\alpha$.

Na comparação dos isótopos de Zr (Figura 2.11), temos um máximo absoluto de $Q_{\alpha} / A_{T}$ em $A_{T}=92$ e outros dois máximos locais em $A_{T}=82$ e $A_{T}=98$. Os três valores de $A_{T}$ mencionados correspondem aos núcleos ${ }^{92} \mathrm{Zr},{ }^{82} \mathrm{Zr}$ e ${ }^{98} \mathrm{Zr}$, os quais possuem as estruturas $\alpha+{ }^{88} \mathrm{Sr}, \alpha+{ }^{78} \mathrm{Sr}$ e $\alpha+{ }^{94} \mathrm{Sr}$, respectivamente. Nos sistemas mencionados, o caroço de ${ }^{88} \mathrm{Sr}$ apresenta a subcamada $1 f_{5 / 2}$ completa para prótons e camada fechada para nêutrons (50 nêutrons), o ${ }^{78} \mathrm{Sr}$ apresenta a subcamada $1 f_{5 / 2}$ completa para prótons e a subcamada $2 p_{1 / 2}$ completa para nêutrons, e o ${ }^{94} \mathrm{Sr}$ apresenta a subcamada $1 f_{5 / 2}$ completa para prótons e a subcamada $2 d_{5 / 2}$ completa para nêutrons (considera-se que a subcamada $2 d_{5 / 2}$ encontra-se abaixo da subcamada $1 g_{7 / 2}[27,28]$ ). Na comparação dos isóbaros de $A_{T}=92$ (Figura 2.12), temos um máximo local de $Q_{\alpha} / A_{T}$ correspondendo ao ${ }^{92} \mathrm{Zr}$. A avaliação simultânea dos isótopos de $\mathrm{Zr}$ e dos isóbaros de $A_{T}=92$ mostra que o ${ }^{92} \mathrm{Zr}$ é um núcleo preferencial para a aglomeração- $\alpha$ e, por este fato, foi considerado o isótopo de Zr mais apropriado para uma análise da estrutura de cluster- $\alpha$.

$\mathrm{Na}$ comparação dos isótopos de $\mathrm{Ru}$ (Figura 2.13), temos um máximo absoluto de $Q_{\alpha} / A_{T}$ em $A_{T}=96$. O núcleo correspondente ${ }^{96} \mathrm{Ru}$ possui a estrutura $\alpha+{ }^{92} \mathrm{Mo}$, onde o caroço de ${ }^{92} \mathrm{Mo}$ apresenta a subcamada $1 g_{9 / 2}$ incompleta para prótons e 


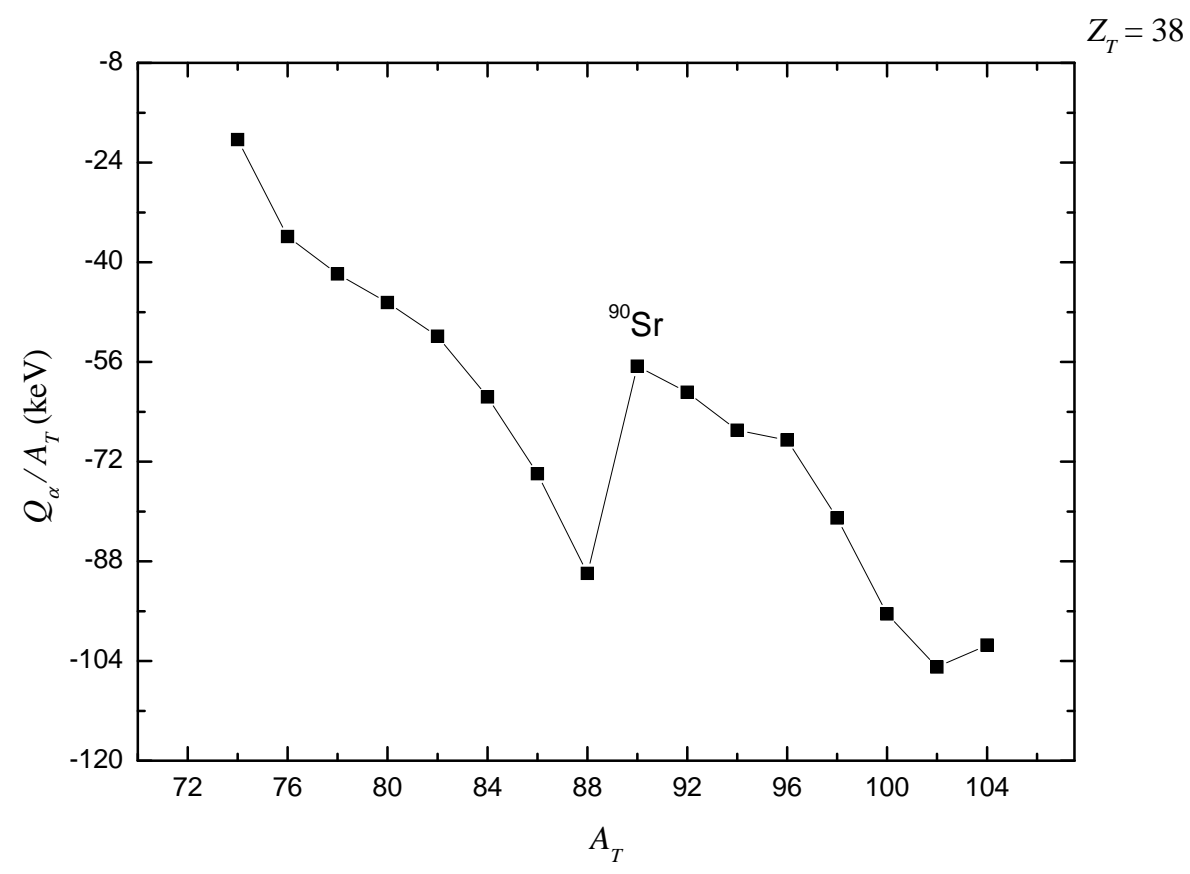

Figura 2.9: Comparação entre os valores de $Q_{\alpha} / A_{T}$ para os isótopos par-par de $\operatorname{Sr}\left(Z_{T}=38\right)$. É indicado o máximo local de $Q_{\alpha} / A_{T}$ correspondente ao ${ }^{90} \mathrm{Sr}$, o qual foi selecionado entre seus isótopos e isóbaros para a análise da sua estrutura $\alpha+$ caroço (ver explicações no texto).

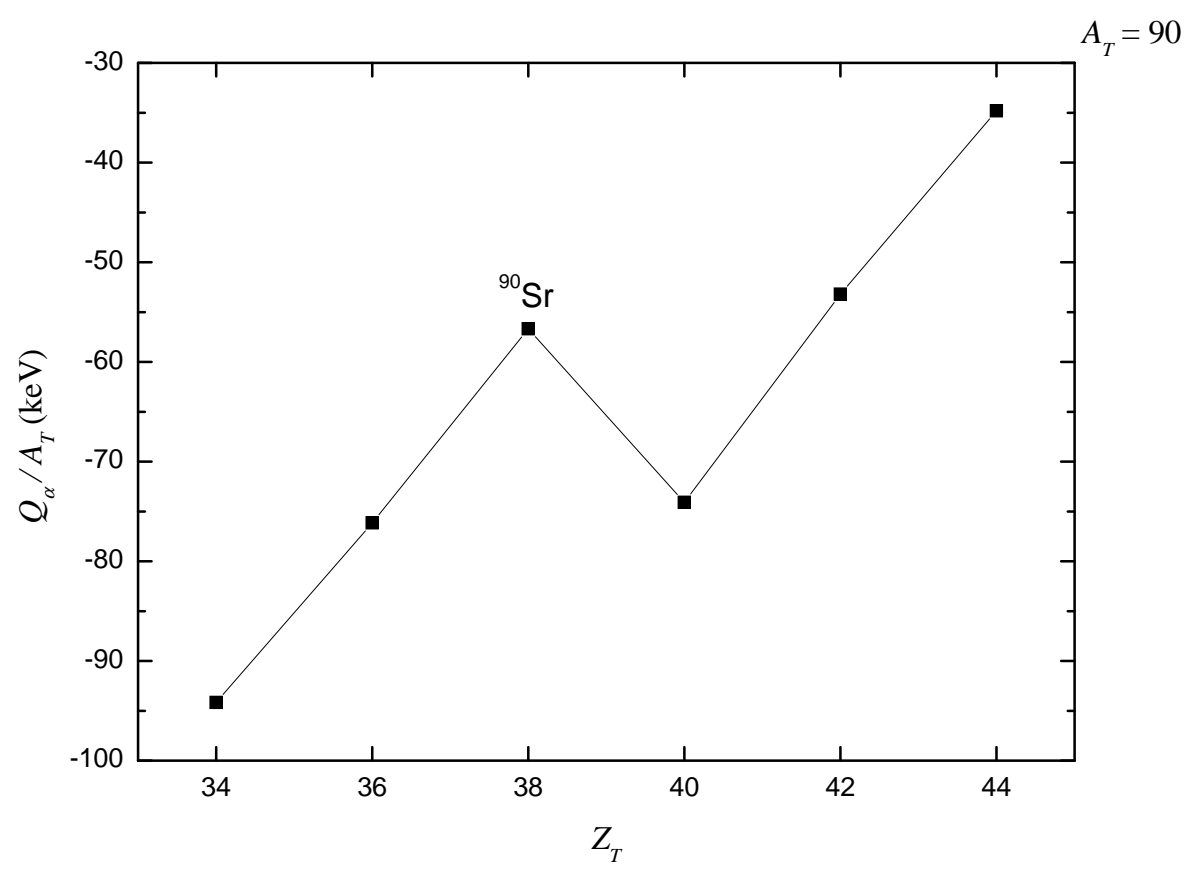

Figura 2.10: Comparação entre os valores de $Q_{\alpha} / A_{T}$ para os isóbaros par-par de $A_{T}=90$. É indicado o máximo local de $Q_{\alpha} / A_{T}$ correspondente ao ${ }^{90} \mathrm{Sr}$, o qual foi selecionado entre seus isótopos e isóbaros para a análise da sua estrutura $\alpha+$ caroço (ver explicações no texto). 


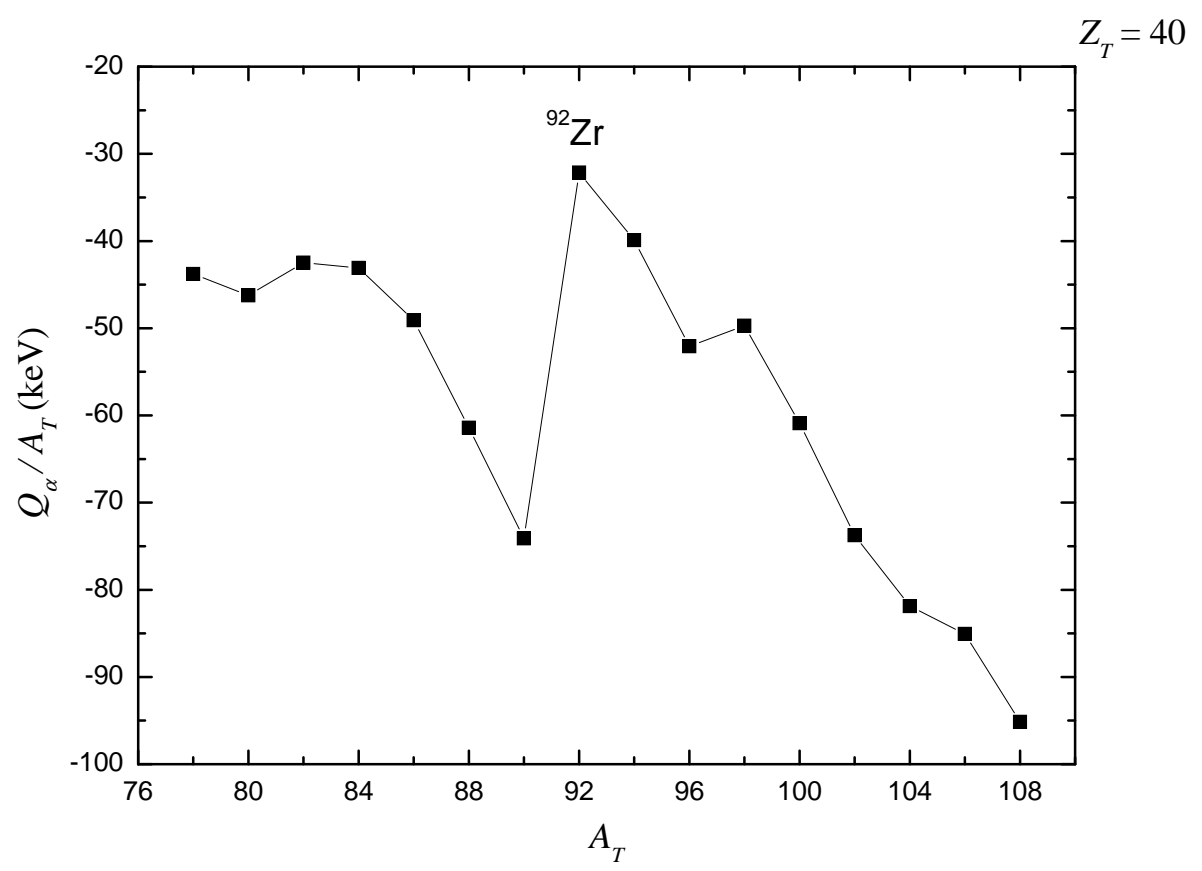

Figura 2.11: Comparação entre os valores de $Q_{\alpha} / A_{T}$ para os isótopos par-par de $\operatorname{Zr}\left(Z_{T}=40\right)$. É indicado o máximo absoluto de $Q_{\alpha} / A_{T}$ correspondente ao ${ }^{92} \mathrm{Zr}$, o qual foi selecionado entre seus isótopos e isóbaros para a análise da sua estrutura $\alpha+$ caroço (ver explicações no texto).

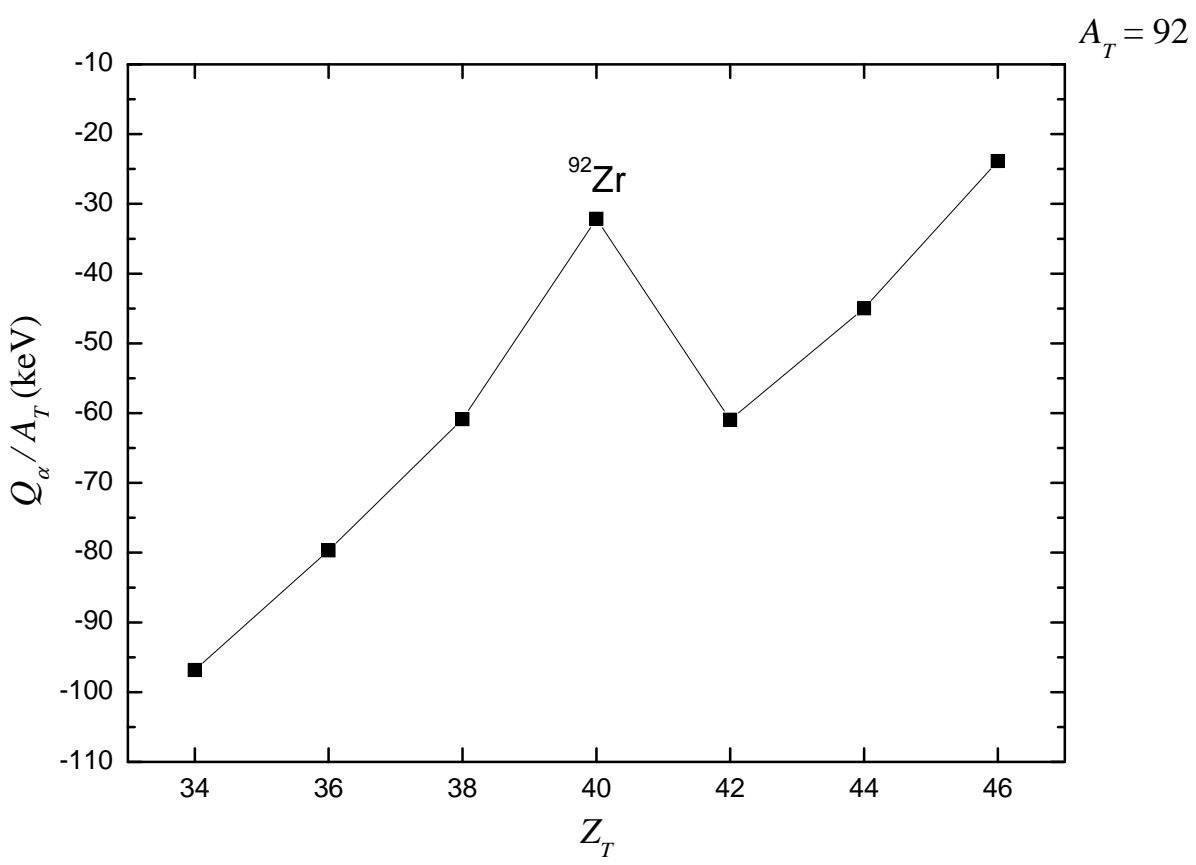

Figura 2.12: Comparação entre os valores de $Q_{\alpha} / A_{T}$ para os isóbaros par-par de $A_{T}=92$. É indicado o máximo local de $Q_{\alpha} / A_{T}$ correspondente ao ${ }^{92} \mathrm{Zr}$, o qual foi selecionado entre seus isótopos e isóbaros para a análise da sua estrutura $\alpha+$ caroço (ver explicações no texto). 


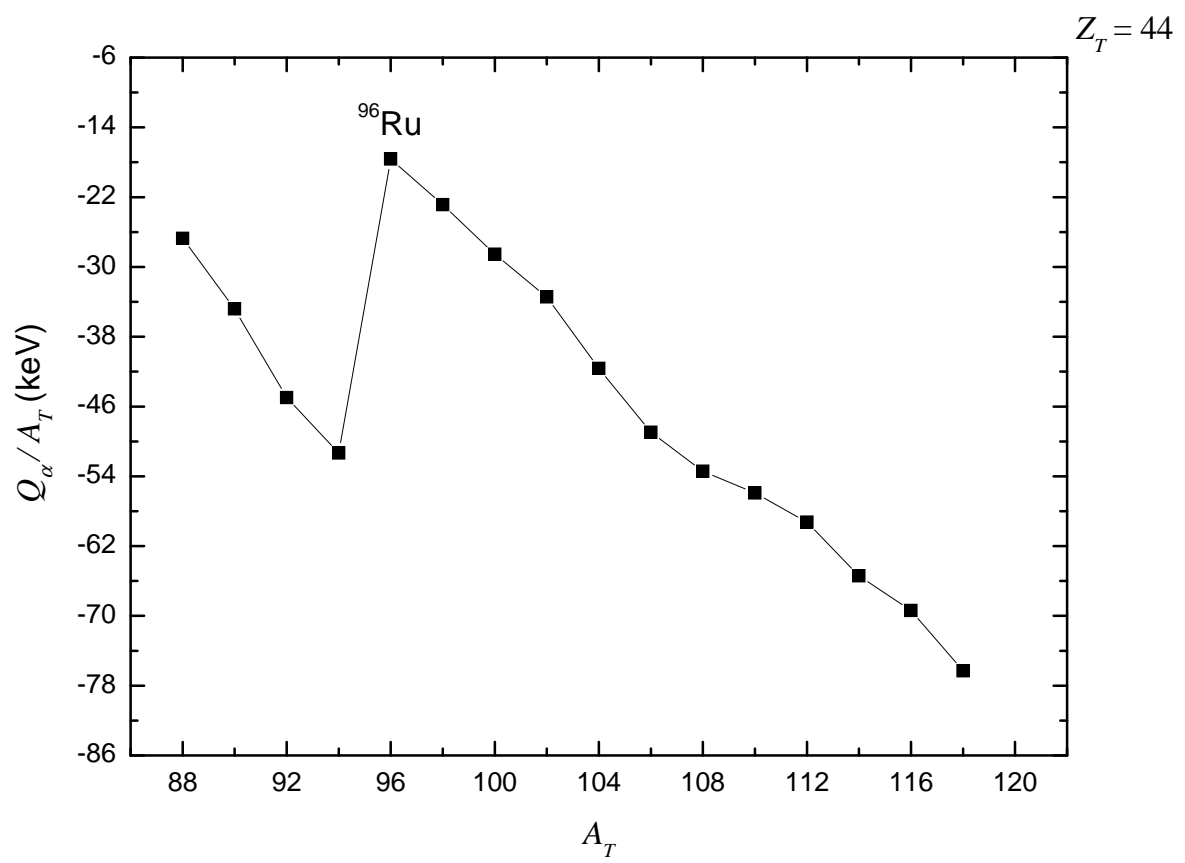

Figura 2.13: Comparação entre os valores de $Q_{\alpha} / A_{T}$ para os isótopos par-par de $\mathrm{Ru}\left(Z_{T}=44\right)$. É indicado o máximo absoluto de $Q_{\alpha} / A_{T}$ correspondente ao ${ }^{96} \mathrm{Ru}$, o qual foi selecionado entre seus isótopos e isóbaros para a análise da sua estrutura $\alpha+$ caroço (ver explicações no texto).

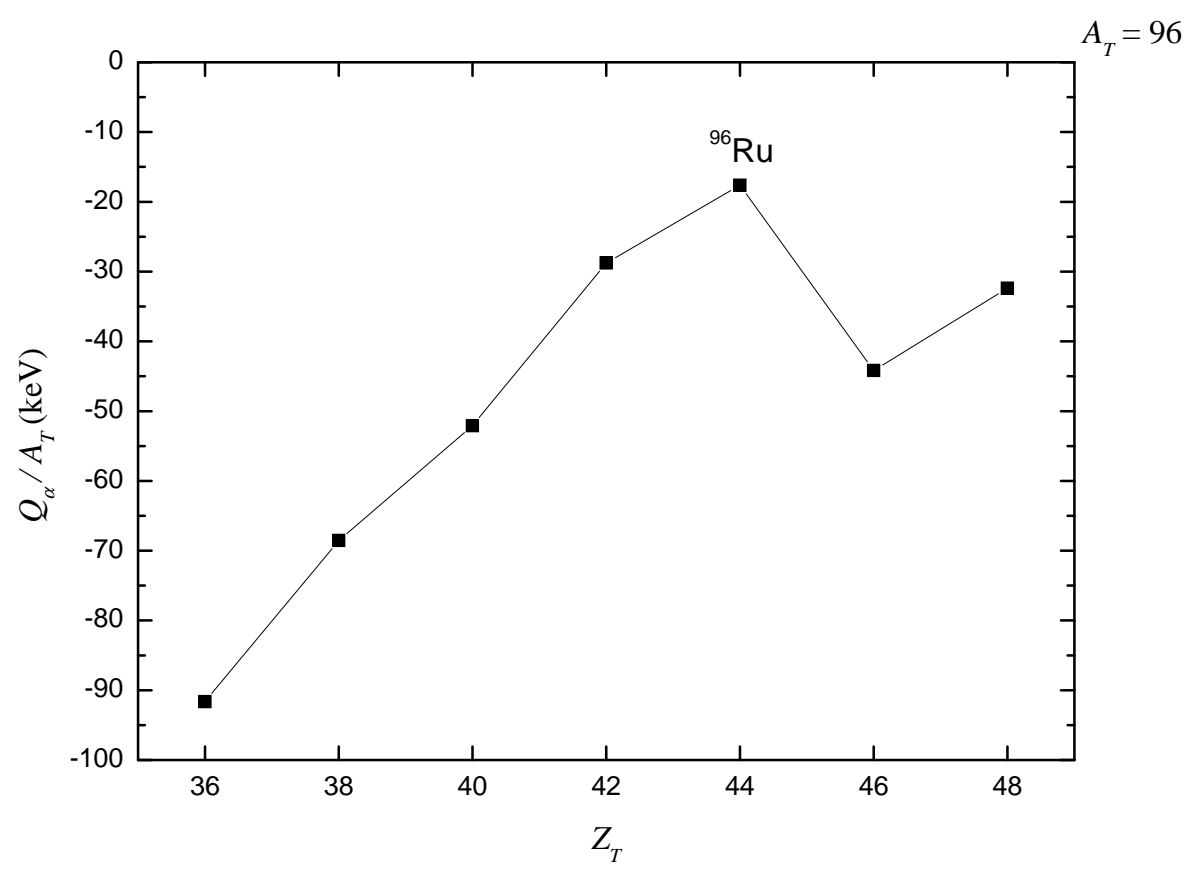

Figura 2.14: Comparação entre os valores de $Q_{\alpha} / A_{T}$ para os isóbaros par-par de $A_{T}=96$. É indicado o máximo absoluto de $Q_{\alpha} / A_{T}$ correspondente ao ${ }^{96} \mathrm{Ru}$, o qual foi selecionado entre seus isótopos e isóbaros para a análise da sua estrutura $\alpha+$ caroço (ver explicações no texto). 


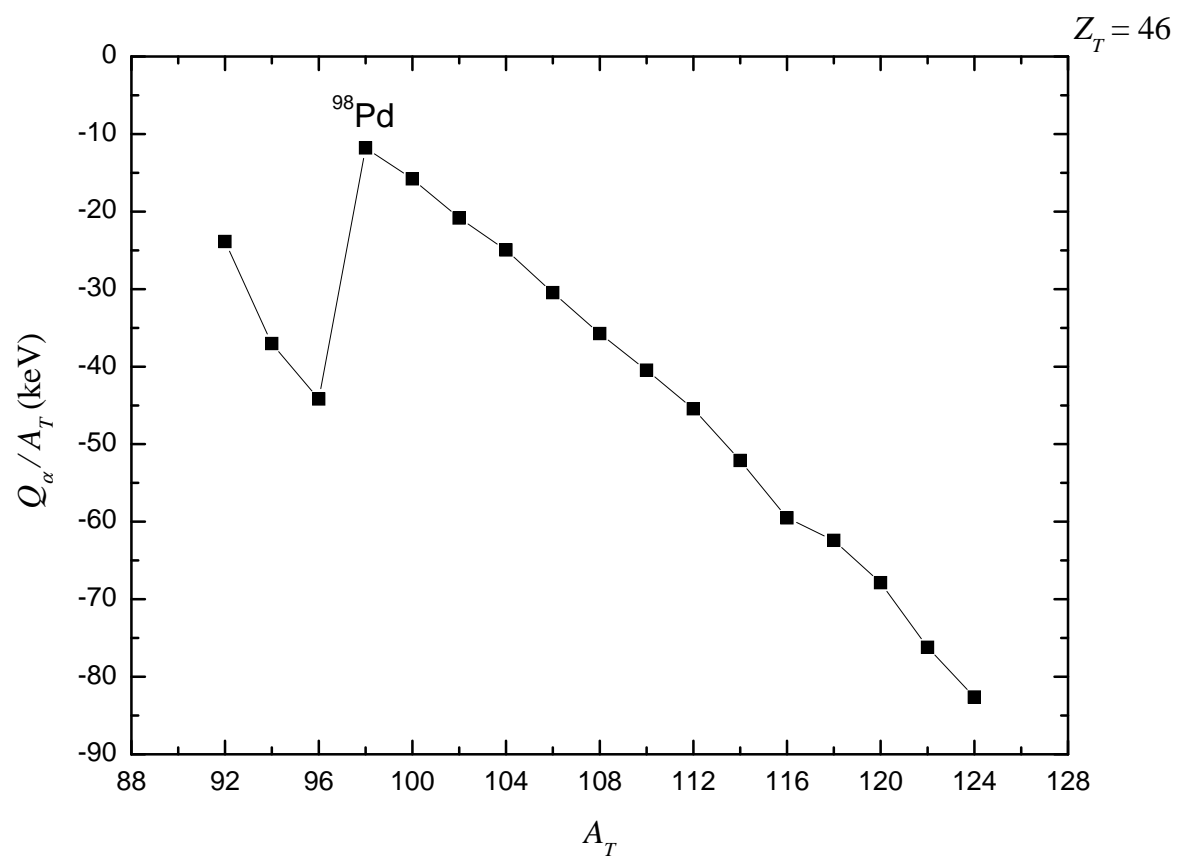

Figura 2.15: Comparação entre os valores de $Q_{\alpha} / A_{T}$ para os isótopos par-par de $\operatorname{Pd}\left(Z_{T}=46\right)$. É indicado o máximo absoluto de $Q_{\alpha} / A_{T}$ correspondente ao ${ }^{98} \mathrm{Pd}$, o qual foi selecionado entre seus isótopos e isóbaros para a análise da sua estrutura $\alpha+$ caroço (ver explicações no texto).

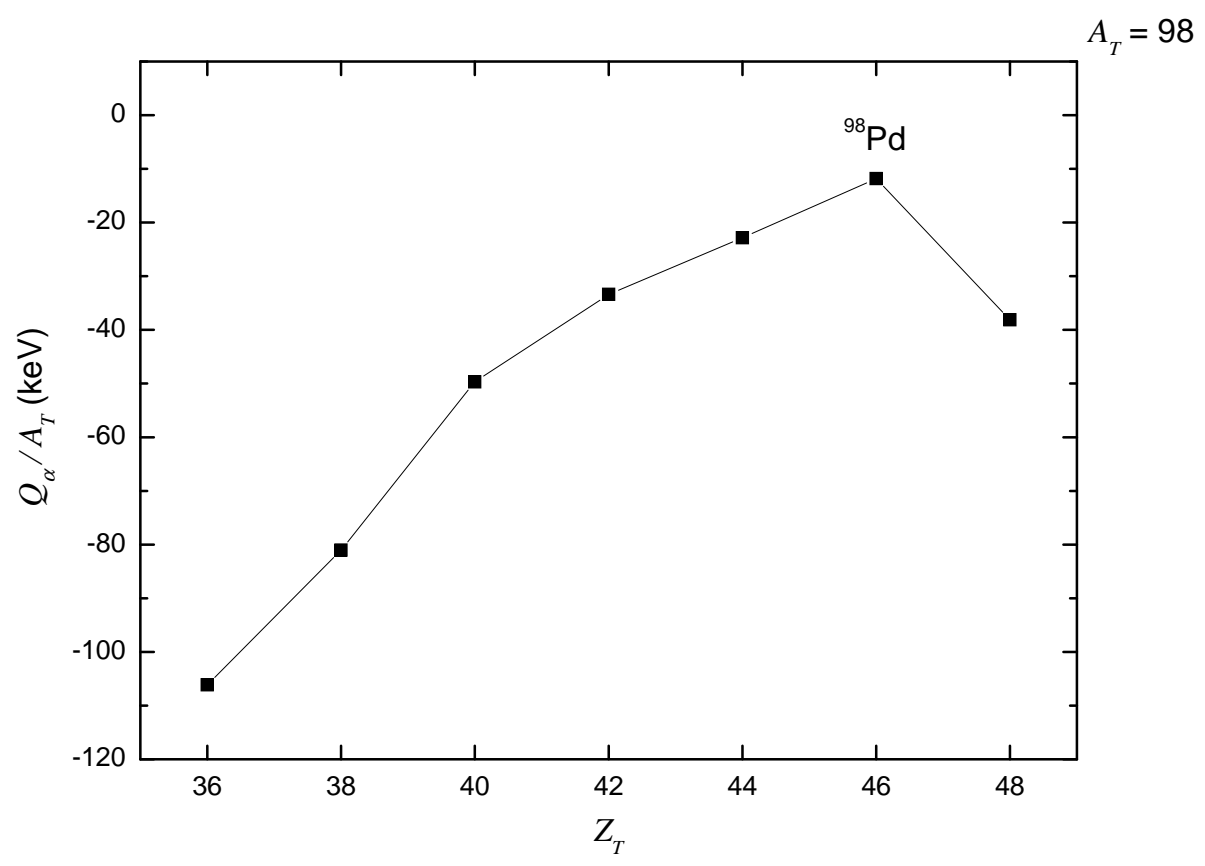

Figura 2.16: Comparação entre os valores de $Q_{\alpha} / A_{T}$ para os isóbaros par-par de $A_{T}=98$. É indicado o máximo absoluto de $Q_{\alpha} / A_{T}$ correspondente ao ${ }^{98} \mathrm{Pd}$, o qual foi selecionado entre seus isótopos e isóbaros para a análise da sua estrutura $\alpha+$ caroço (ver explicações no texto). 
camada fechada para nêutrons (50 nêutrons). Na comparação dos isóbaros de $A_{T}=96$ (Figura 2.14), temos um máximo absoluto de $Q_{\alpha} / A_{T}$ correspondendo ao ${ }^{96} \mathrm{Ru}$. Mostra-se que o ${ }^{96} \mathrm{Ru}$ é o núcleo preferencial para a aglomeração- $\alpha$ quando é comparado aos seus isótopos e isóbaros simultaneamente e, por este fato, foi considerado o isótopo de $\mathrm{Ru}$ mais apropriado para uma análise da estrutura de cluster- $\alpha$.

Repetindo a mesma comparação para os isótopos de Pd (Figura 2.15), temos um máximo absoluto de $Q_{\alpha} / A_{T}$ em $A_{T}=98$. O núcleo correspondente ${ }^{98} \mathrm{Pd}$ possui a estrutura $\alpha+{ }^{94} \mathrm{Ru}$, onde o caroço de ${ }^{94} \mathrm{Ru}$ apresenta a subcamada $1 g_{9 / 2}$ incompleta para prótons e camada fechada para nêutrons (50 nêutrons). Na comparação dos isóbaros de $A_{T}=98$ (Figura 2.16), temos um máximo absoluto de $Q_{\alpha} / A_{T}$ correspondendo ao ${ }^{98} \mathrm{Pd}$. Mostra-se então que o ${ }^{98} \mathrm{Pd}$ é o núcleo preferencial para a aglomeração- $\alpha$ quando é comparado aos seus isótopos e isóbaros simultaneamente e, por este fato, foi considerado o isótopo de Pd mais apropriado para uma análise da estrutura de cluster- $\alpha$.

Após as comparações entre os núcleos dos diferentes conjuntos de isótopos e isóbaros da região especificada, nota-se novamente que quase todos os máximos absolutos e locais de $Q_{\alpha} / A_{T}$ estão associados a núcleos cujas estruturas $\alpha+$ caroço são constituídas de caroços de camada fechada ou subcamada completa de prótons e/ou nêutrons (sendo o ${ }^{74} \mathrm{Sr}$ a única exceção entre os núcleos mencionados). Portanto, igualmente ao que foi constatado para os núcleos ${ }^{20} \mathrm{Ne},{ }^{44} \mathrm{Ti},{ }^{94} \mathrm{Mo} \mathrm{e}{ }^{212} \mathrm{Po}$, o critério de comparação de $Q_{\alpha} / A_{T}$ demonstra claramente os efeitos de modelo de camadas na determinação da estrutura $\alpha+$ caroço mais favorável. Nos casos dos núcleos selecionados como preferenciais para a aglomeração- $\alpha$ na região do Mo $\left({ }^{90} \mathrm{Sr},{ }^{92} \mathrm{Zr},{ }^{94} \mathrm{Mo},{ }^{96} \mathrm{Ru}\right.$ e $\left.{ }^{98} \mathrm{Pd}\right)$, verifica-se que todos possuem em comum caroços nucleares com o número mágico de nêutrons $N_{\text {car }}=50$. Portanto, os resultados do critério de comparação de $Q_{\alpha} / A_{T}$ indicam que, na região de massa do Mo, os núcleos com a estrutura $\alpha+$ caroço mais favorável são aqueles com $N_{T}=N_{\text {car }}+N_{\alpha}=52$. Tal indicação concorda com as previsões de P. Mohr [17], o qual considera que a presença do caroço nuclear com $N_{\text {car }}=50$ é um requisito suficiente para a aplicação do modelo de cluster- $\alpha$ nos núcleos desta região de massa. 


\section{Capítulo 3}

\section{Determinação das bandas de estado fundamental dos sistemas $\alpha+$ caroço}

Este capítulo descreve os métodos usados para o cálculo das bandas de estado fundamental dos sistemas $\alpha+$ caroço e das funções de onda radiais associadas. As bandas calculadas são comparadas a níveis de energia experimentais e a qualidade dos resultados é discutida. Detalhes referentes aos parâmetros do potencial de interação $\alpha+$ caroço e do número quântico global $G$ também são comentados.

\subsection{Descrição inicial do sistema $\alpha+$ caroço}

Uma tarefa inicial para a resolução do sistema $\alpha+$ caroço é a definição das equações que descrevem os auto-estados do sistema. Assim, seguimos o procedimento no qual as funções de onda e o espectro de energias do sistema são determinados diretamente pela resolução da equação de Schrödinger independente do tempo

$$
\nabla^{2} \psi+\frac{2 \mu}{\hbar^{2}}[E-V(r)] \psi=0
$$

onde $\mu$ é a massa reduzida do sistema, dada por

$$
\mu=\frac{m_{\alpha} m_{\mathrm{car}}}{m_{\alpha}+m_{\mathrm{car}}}
$$

e $V(r)$ é o potencial de interação $\alpha+$ caroço. Deve-se considerar as características do sistema para se justificar o uso simples da eq. (3.1). Em concordância com as discussões do Capítulo 1, os únicos termos relevantes na função Hamiltoniana do sistema são aqueles relacionados ao movimento relativo entre os centros de massa 
do cluster- $\alpha$ e do caroço. Com isto, as propriedades do sistema são determinadas através das funções de onda associadas ao movimento relativo $\alpha$-caroço . A eq. (3.1) é usada para o cálculo das funções de onda $\psi(r, \theta, \phi)$, sendo $r, \theta$ e $\phi$ as coordenadas do movimento relativo.

Para que sejam obtidas as soluções $\psi(r, \theta, \phi)$, deve ser aplicado o método de separação de variáveis em (3.1). As soluções terão a forma [32]:

$$
\psi_{N, L, M}(r, \theta, \phi)=\frac{1}{r} u_{N, L}(r) Y_{L, M}(\theta, \phi) .
$$

Como o potencial $V(r)$ possui simetria radial, a parte angular $Y_{L, M}(\theta, \phi)$ será dada pelos harmônicos esféricos, onde os números quânticos $L$ e $M$ são aqueles usados na definição do momento angular orbital. A parte $u_{N, L}(r)$ é a função radial que depende dos números quânticos $N$ e $L$, onde $N$ é o número de nós internos desta função radial. Como a parte angular já é conhecida analiticamente, resta determinar a parte radial $u_{N, L}(r)$ através da resolução da equação radial

$$
\frac{d^{2} u}{d r^{2}}+\left\{\frac{2 \mu}{\hbar^{2}}[E-V(r)]-\frac{L(L+1)}{r^{2}}\right\} u=0
$$

que é resultante da aplicação do método de separação de variáveis em (3.1). A resolução de (3.4) nos fornece o espectro de energias do sistema e as auto-funções associadas a estas energias.

\subsection{Método numérico aplicado à resolução do sistema}

Diante do problema estabelecido, é necessário o uso de um método numérico para a resolução da eq. (3.4). Após uma pesquisa pelas formas mais usuais, foi escolhido o método Runge-Kutta-Fehlberg (RKF) para equações diferenciais de segunda ordem. Este método produz uma solução preliminar $f(x)$ gerada por uma aproximação do tipo Runge-Kutta de quinta ordem, porém, o erro cometido na solução efetiva da equação é definido como a diferença entre os resultados obtidos pelas aproximações de quinta e quarta ordem. Desta forma, o método RKF produz uma solução efetiva $f(x)$ relacionada à aproximação de quarta ordem. O método RKF diferencia-se do Runge-Kutta tradicional por incluir um processo de refinamento da solução onde o passo de integração $h$ é ajustado conforme são calculados consecutivamente os pontos da função no processo iterativo. O propósito deste refinamento é adaptar $h$ para que o erro numérico cometido na derivada da função nunca ultrapasse um limite pré-estabelecido em cada passo da integração. O recurso do refinamento torna-se útil para uma padronização da grandeza do erro cometido ao longo da função $f(x)$, além de evitar o aumento excessivo do erro 
em trechos da função onde há um comportamento exponencial. O método RKF é descrito em detalhes na Ref. [31]. Alguns testes para a verificação da eficiência do método RKF são mostrados na Ref. [33], onde a eq. (3.4) é resolvida com um potencial do tipo oscilador harmônico com $\hbar \omega=41 A^{-1 / 3} \mathrm{MeV}$.

Nos casos onde a eq. (3.4) foi resolvida com o método RKF para a determinação dos auto-valores de energia dos diferentes sistemas $\alpha+$ caroço, o erro numérico máximo $\varepsilon$ (erro absoluto associado a cada passo de integração) para a derivada de $u(r)$ foi estabelecido como $3.5 \times 10^{-8} \mathrm{fm}^{-3 / 2}$ dentro do referido método, enquanto o passo $h$ foi limitado por um valor mínimo de $10^{-4} \mathrm{fm}$ e um valor máximo de $10^{-2} \mathrm{fm}$.

O procedimento seguinte é a aplicação do método RKF na resolução da eq. (3.4) com o potencial de interação $\alpha+$ caroço $V(r)$. Porém, são necessárias adaptações para este caso. Na situação onde a equação é resolvida com passo $h$ positivo, as soluções $u(r)$ podem apresentar um comportamento de divergência em $r \gg 0$ devido a pequenas imprecisões provocadas pelo próprio método numérico durante o processo iterativo. Além disso, os auto-valores de energia do sistema podem ser determinados com pequenos erros, também contribuindo para o efeito de divergência (o efeito de divergência em $r \gg 0$ é analisado em detalhes na Ref. [33]).

O procedimento escolhido para evitar a divergência da função radial consiste em calcular separadamente as partes interna e externa da função $u(r)$ em relação ao poço de potencial efetivo. O método RKF com passo positivo é bastante eficiente na parte interna do poço de potencial efetivo e na parte externa mais próxima de $r=0$, já que não há o efeito de divergência nestas regiões. Deste modo, a parte interna de $u(r)$ pode ser calculada com passo positivo e condição inicial em $r_{0}=0$ ou próximo de 0 . Quanto à parte externa de $u(r)$ além da região do poço, pode-se determiná-la usando o método RKF com passo negativo e condição inicial em $r_{0} \gg 0$, evitando assim o efeito de divergência nesta região. Para estados ligados (abaixo do limiar de separação $\alpha+$ caroço), as condições iniciais para a função $u(r)$ e sua derivada $\left(u\left(r_{0}\right)\right.$ e $\left.u^{\prime}\left(r_{0}\right)\right)$ devem ser escolhidas adequadamente em $r_{0}=0$ e $r_{0} \gg 0$ para que sejam compatíveis com as condições de contorno $u(0)=0$ e $u(r \rightarrow \infty)=0$, respectivamente.

A seguir, é descrito como foram delimitadas as regiões interna e externa relativas ao poço de potencial efetivo. A Figura 3.1 mostra o comportamento do potencial efetivo e as regiões interna e externa em relação à fronteira do poço na energia $E$. Desta forma, é convencionado que a região I compreende o trecho interno do poço de potencial efetivo e o trecho externo mais próximo de $r=0$, enquanto a região II compreende toda a parte externa restante além da fronteira do poço. Esta fronteira é delimitada pelo raio $r_{\mathrm{f}}$ que varia de acordo com a energia $E$. A solução da equação radial (3.4) na região I é chamada $u_{\mathrm{I}}(r)$, enquanto na região II é chamada $u_{\mathrm{II}}(r)$. Estas soluções ainda não incluem constantes de normalização, 


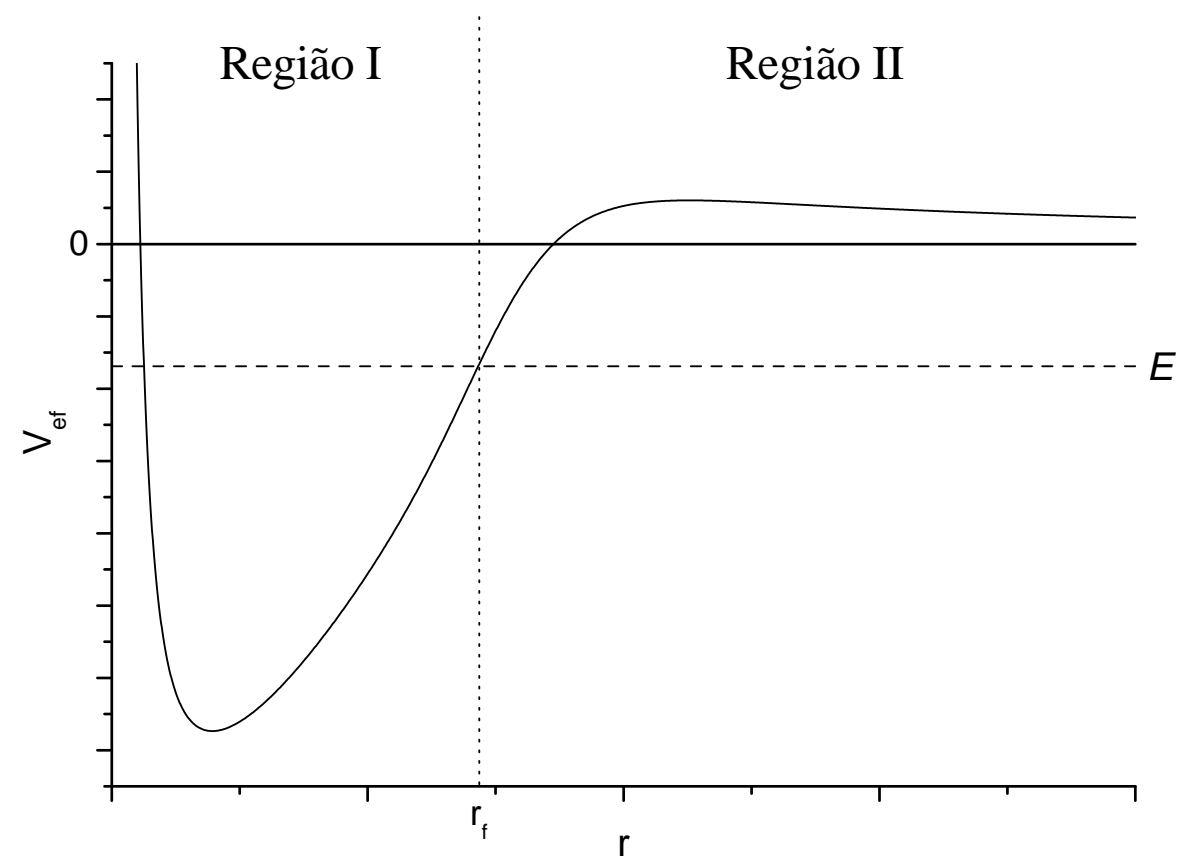

Figura 3.1: Representação em escala arbitrária do potencial efetivo $V_{\text {ef }}(r)$ com a delimitação entre as regiões I e II no caso onde a equação radial (3.4) é resolvida com energia $E$. As soluções $u_{\mathrm{I}}(r)$ e $u_{\mathrm{II}}(r)$, obtidas respectivamente nas regiões I e II, são calculadas de forma independente. $\mathrm{O}$ raio de fronteira $r_{\mathrm{f}}$ delimita as duas regiões.

como é justificado posteriormente nesta seção.

Um procedimento adicional escolhido para a resolução do sistema $\alpha+$ caroço consiste no uso de condições iniciais para o método RKF as quais se baseiam nas soluções analíticas da equação radial para o poço de potencial quadrado. Tal procedimento é justificado através da aproximação do potencial intercluster como uma justaposição de potenciais do tipo degrau que acompanham a variação de profundidade de $V(r)$, desde que cada um destes potenciais degrau seja bastante estreito na sua extensão $\Delta r$. Contudo, esta aproximação é utilizada apenas como condição inicial em $r_{0}$ para a resolução da eq. (3.4) com o próprio potencial intercluster $V(r)$. Desta forma, $V(r)$ é aproximado em $r_{0}$ como $V_{0}=V\left(r_{0}\right)$.

A aproximação de poço quadrado é aplicada tanto para $r_{0}=0$ (ou $r_{0} \approx 0$ ) quanto para $r_{0} \gg 0$ (no caso de estados ligados ou quase-ligados), considerando as devidas adaptações em cada situação. Tal aproximação deve reproduzir com suficiente acurácia as condições iniciais ideais, isto é, aquelas que resultam numa derivada logarítmica inicial $u^{\prime}\left(r_{0}\right) / u\left(r_{0}\right)$ compatível com a verdadeira solução da eq. (3.4) em $r_{0}$, levando-se em conta as condições de contorno $u(0)=0 \mathrm{e}$ $u(r \rightarrow \infty)=0$ e a energia $E$ do sistema.

No cálculo de $u_{\mathrm{I}}(r)$ com $L=0$, a condição inicial de poço quadrado pode ser aplicada em $r_{0}=0$, enquanto que, para $L>0$, a condição inicial de poço quadrado 
deve ser aplicada num raio $r_{0}$ não nulo, mas próximo de zero. Tal restrição para $L>0$ ocorre porque o termo associado à barreira centrífuga na eq. (3.4) provoca uma divisão do tipo $L(L+1) / 0$ caso seja usado $r_{0}=0$, o que é impraticável do ponto de vista computacional. Neste trabalho, foi empregado o valor $r_{0}=0.003 \mathrm{fm}$ para todos os cálculos de $u_{\mathrm{I}}(r)$, mesmo para $L=0$, visando uma padronização dos cálculos computacionais realizados. No cálculo de $u_{\mathrm{II}}(r)$, onde a condição inicial deve ser aplicada num raio $\gg 0$, foi empregado o valor $r_{0}=100 \mathrm{fm}$ para todos os cálculos deste trabalho envolvendo estados ligados. Os valores numéricos adotados para $r_{0}$ foram escolhidos com base em estudos anteriores do sistema $\alpha+{ }^{90} \mathrm{Zr}$ na Ref. [33].

Em concordância com as descrições dos parágrafos anteriores e usando como referência as soluções analíticas $u_{p . q .}(r)$ para o poço quadrado (mais precisamente, para um potencial constante $V_{0}=V\left(r_{0}\right)$ ) [34], as condições iniciais aplicadas na determinação dos estados ligados ou quase-ligados são

$$
u_{\mathrm{I}}\left(r_{0}\right)=A r_{0} j_{L}\left(\kappa r_{0}\right) \quad \text { na região I }
$$

e

$$
u_{\mathrm{II}}\left(r_{0}\right)=B r_{0} h_{L}^{(1)}\left(i \alpha r_{0}\right) \quad \text { na região II, }
$$

onde

$$
\kappa^{2}=\frac{2 \mu}{\hbar^{2}}\left(E-V\left(r_{0}\right)\right) \quad \text { e } \quad \alpha^{2}=-\frac{2 \mu}{\hbar^{2}}\left(E-V\left(r_{0}\right)\right) .
$$

As funções $j_{L}$ e $h_{L}^{(1)}$ são, respectivamente, as funções de Bessel esféricas e as funções de Hankel esféricas do primeiro tipo. As condições iniciais para as derivadas $u_{\mathrm{I}}^{\prime}\left(r_{0}\right)$ e $u_{\mathrm{II}}^{\prime}\left(r_{0}\right)$ são obtidas com a simples derivação das funções analíticas $u_{p . q .}(r)$. Os coeficientes $A$ e $B$ devem ser calculados de tal forma que seja mantida a continuidade da solução completa $u(r)$ no raio $r_{\mathrm{f}}$, resultando em $u_{\mathrm{I}}\left(r_{\mathrm{f}}\right)=u_{\mathrm{II}}\left(r_{\mathrm{f}}\right)$. A solução completa então será

$$
u(r)=\left\{\begin{array}{l}
u_{\mathrm{I}}(r) \quad \text { para } \quad r \leq r_{\mathrm{f}} \\
u_{\mathrm{II}}(r) \text { para } r>r_{\mathrm{f}}
\end{array} .\right.
$$

A solução $u(r)$ ainda não é normalizada, pois as condições iniciais dadas em (3.5) e (3.6) não incluem coeficientes com esta função. Primeiramente, é necessária a determinação dos auto-valores de energia para que as soluções correspondentes $u(r)$ sejam calculadas e em seguida normalizadas.

O desconhecimento dos auto-valores de energia do sistema $\alpha+$ caroço torna necessário determiná-los por tentativas, isto é, fazendo uma varredura em todo o intervalo de energia onde devem estar localizados os auto-estados do sistema. Uma 
certa energia $E$ é considerada um dos auto-valores quando resulta na continuidade da derivada logarítmica de $u(r)$ em $r_{\mathrm{f}}$. Isto pode ser descrito em termos de $u_{\mathrm{I}}(r)$ e $u_{\mathrm{II}}(r)$ por

$$
\left.\frac{1}{u_{\mathrm{I}}(r)}\left(\frac{d u_{\mathrm{I}}(r)}{d r}\right)\right|_{r=r_{\mathrm{f}}}=\left.\frac{1}{u_{\mathrm{II}}(r)}\left(\frac{d u_{\mathrm{II}}(r)}{d r}\right)\right|_{r=r_{\mathrm{f}}} .
$$

O processo de varredura é executado através da resolução numérica da eq. (3.4) com diferentes valores de energia, os quais se distanciam por um passo constante $\Delta E$. Desta forma, é previsto que uma varredura de passo $\Delta E$ deve resultar em auto-energias com erros da ordem de $(\Delta E) / 2$. Levando em conta a precisão adotada pela Ref. [33] na determinação dos níveis de energia do sistema $\alpha+{ }^{90} \mathrm{Zr}$, foi usado $\Delta E=0.01 \mathrm{MeV}$ em todas as varreduras referentes às bandas de energia dos sistemas $\alpha+$ caroço, o que fornece um grau de precisão suficiente para os propósitos seguintes deste trabalho. Do ponto de vista do cálculo numérico, $E$ é estabelecido como um dos auto-valores de energia quando o módulo da diferença entre os dois membros da eq. (3.9) atinge um mínimo dentro da faixa submetida à varredura.

\subsection{Tratamento para estados quase-ligados}

Os procedimentos descritos na seção anterior são usados em princípio para o cálculo de estados ligados $(E \leq 0)$, isto é, abaixo do limiar $\alpha+$ caroço. Contudo, os mesmos procedimentos podem ser utilizados para o cálculo de estados quaseligados com algumas adaptações.

A barreira de potencial efetivo formada por $V_{\text {ef }}(r)$ possui largura limitada em energias acima do limiar do sistema $\alpha+$ caroço; desta forma, os estados que se localizam muitos $\mathrm{MeV}$ acima do limiar e próximos do topo da barreira devem ser tratados como níveis ressonantes, conforme as discussões da Subseção 5.2.2. Entretanto, os níveis que se encontram pouco acima do limiar e muito abaixo do topo da barreira podem ser tratados por uma aproximação de estado ligado. Para a aplicação desta aproximação, deve-se verificar a largura da barreira de potencial efetivo no intervalo de energia onde os níveis devem estar localizados e o quanto este intervalo está abaixo do topo da barreira. Considerando os núcleos de massa intermediária escolhidos neste trabalho para o estudo da estrutura de cluster- $\alpha$ $\left({ }^{90} \mathrm{Sr},{ }^{92} \mathrm{Zr},{ }^{94} \mathrm{Mo},{ }^{96} \mathrm{Ru}\right.$ e $\left.{ }^{98} \mathrm{Pd}\right)$, verifica-se que muitos níveis experimentais de suas bandas de estado fundamental encontram-se acima do limiar $\alpha+$ caroço, principalmente aqueles com spins mais altos. Apesar deste fato, tais níveis situam-se em energias onde a barreira de potencial efetivo ainda é muito extensa (a extensão varia entre algumas dezenas de fm, para os estados de mais alto spin, até a ordem de centenas de $\mathrm{fm}$, para os estados de mais baixo spin acima do limiar) e, além disso, muitos destes níveis encontram-se vários $\mathrm{MeV}$ abaixo do topo da barreira 
efetiva (a distância em relação ao topo da barreira varia entre a ordem de $10 \mathrm{MeV}$, para os estados acima do limiar com spin mais baixo, até algumas dezenas de $\mathrm{MeV}$, para os estados de mais alto spin). As características mencionadas mostram que a aproximação de estado ligado é bastante apropriada para os estados acima do limiar e pertencentes às bandas de estado fundamental.

A aproximação de estado ligado consiste em aplicar os mesmos procedimentos já usados na resolução da equação radial para níveis ligados, contudo, considerando uma barreira de potencial com extensão limitada. Como se deseja que a função $u(r)$ apresente a característica de estado ligado mesmo em $E>0$, ela deve apresentar um comportamento exponencial decrescente fora do poço de potencial efetivo, isto é, o mesmo comportamento verificado para $E<0$. A função $u(r)$ pode apresentar naturalmente esta característica exponencial nos trechos onde $E<V_{\text {ef }}(r)$.

Dentro destas condições, é estabelecido que a condição inicial do método RKF na região II deve ser aplicada num raio $r_{0} \gg 0$ onde $E<V_{0}$. Apesar de $r_{0}$ estar restrito à região classicamente proibida que se encontra dentro da barreira de potencial, ainda é possível aplicá-lo num raio suficientemente distante do poço atrativo quando $E$ está muito abaixo do topo da barreira efetiva, de modo a manter a aproximação de poço quadrado satisfatória em $r_{0}$. A varredura de energia então é aplicada acima de $E=0$ e as condições iniciais do método RKF continuam baseadas em (3.5) e (3.6).

\subsection{Cálculo das bandas de estado fundamental}

O processo de varredura de energia descrito nas Seções 3.2 e 3.3 foi usado para a determinação das bandas de estado fundamental dos sistemas $\alpha+{ }^{86} \mathrm{Kr}, \alpha+{ }^{88} \mathrm{Sr}$, $\alpha+{ }^{90} \mathrm{Zr}, \alpha+{ }^{92} \mathrm{Mo} \mathrm{e} \alpha+{ }^{94} \mathrm{Ru}$ associados aos núcleos ${ }^{90} \mathrm{Sr},{ }^{92} \mathrm{Zr},{ }^{94} \mathrm{Mo},{ }^{96} \mathrm{Ru}$ e ${ }^{98} \mathrm{Pd}$, respectivamente. O potencial $V(r)$ descrito na Seção 1.3 foi aplicado na eq. (3.4) para o cálculo dos níveis de energia dos diferentes sistemas. No entanto, deve-se discutir a importância dos parâmetros do potencial $V(r)$ e do número quântico global $G$ no cálculo de tais níveis.

\subsubsection{Identificação do número quântico $G$ para os sistemas $\alpha+$ caroço}

Como é discutido na Seção 1.2, o número quântico $G$ caracteriza as bandas de estados do sistema e está diretamente relacionado com os orbitais ocupados pelos núcleons do cluster- $\alpha$. Considera-se que os 2 prótons e 2 nêutrons constituintes do cluster- $\alpha$ são os núcleons de valência do sistema, ocupando os orbitais de energia mais alta. Com isto, nos casos dos núcleos ${ }^{90} \mathrm{Sr},{ }^{92} \mathrm{Zr},{ }^{94} \mathrm{Mo},{ }^{96} \mathrm{Ru}$ e ${ }^{98} \mathrm{Pd}$ em seus estados fundamentais, verifica-se que os 2 nêutrons do cluster- $\alpha$ estão situados na 
camada $3 s 2 d 1 g$ (os diferentes diagramas de energia da literatura [25, 26, 27, 28, 29] indicam que os 2 nêutrons devem ocupar orbitais nas subcamadas $1 g_{7 / 2}$ ou $2 d_{5 / 2}$ ). Nos núcleos ${ }^{90} \mathrm{Sr}$ e ${ }^{92} \mathrm{Zr}$ em seus estados fundamentais, os 2 prótons do cluster$\alpha$ estão situados na camada $2 p 1 f$ (os diferentes diagramas de energia indicam a ocupação de orbitais em $1 f_{5 / 2}$ e $2 p_{1 / 2}$ ), enquanto que nos núcleos ${ }^{94} \mathrm{Mo},{ }^{96} \mathrm{Ru}$ e ${ }^{98} \mathrm{Pd}$ em seus estados fundamentais, os 2 prótons do cluster- $\alpha$ estão situados na camada $3 s 2 d 1 g$ (os diferentes diagramas de energia indicam a ocupação de orbitais em $1 g_{9 / 2}$ ). Usando a condição de Wildermuth (ver eq. (1.1)), a qual relaciona $G$ com o número de nós internos $n_{i}$ e o momento angular $l_{i}$ associados aos orbitais dos núcleons do cluster- $\alpha$, é possível calcular o número $G$ que identifica a banda de estado fundamental de cada núcleo estudado. Assim, os valores de $G$ obtidos são:

$$
\begin{gathered}
G=14 \quad \text { para }{ }^{90} \mathrm{Sr} \mathrm{e}{ }^{92} \mathrm{Zr}, \\
G=16 \quad \text { para }{ }^{94} \mathrm{Mo},{ }^{96} \mathrm{Ru} \mathrm{e}{ }^{98} \mathrm{Pd} .
\end{gathered}
$$

O número quântico $G$ determina o número de nós internos $N$ da função radial $u_{N, L}(r)$ a partir de um dado $L$, permitindo identificar as funções radiais correspondentes aos estados da banda.

Diferentes cálculos na literatura sobre a estrutura de cluster- $\alpha$ no ${ }^{94} \mathrm{Mo}[3,4,5]$ mostram que o número $G=16$ é adequado para uma reprodução satisfatória dos níveis de energia experimentais da banda de estado fundamental do ${ }^{94} \mathrm{Mo}$.

\subsubsection{Ajuste do parâmetro $R$ para os sistemas $\alpha+$ caroço}

O potencial de interação $\alpha+$ caroço $V(r)$ apresenta os parâmetros $V_{0}, a, b$ e $R$ (ver eq. (1.5)), sendo que $V_{0}, a$ e $b$ são considerados parâmetros fixos. Deve-se destacar que os parâmetros $V_{0}, a$ e $b$ foram ajustados para a descrição conjunta de sistemas $\alpha+$ caroço de diferentes regiões de massa, de modo que, neste trabalho, o princípio de manter os três parâmetros como fixos foi mantido para a descrição geral das bandas de estado fundamental dos cinco núcleos estudados. O parâmetro $R$ é aquele que permite adaptar o potencial $V(r)$ para cada sistema $\alpha+$ caroço.

Seguindo o procedimento adotado na Ref. [3] para o mesmo potencial $V(r)$, o parâmetro $R$ foi ajustado com uma precisão de $10^{-3} \mathrm{fm}$ para reproduzir com o menor erro possível a energia do estado $4^{+}$da banda de estado fundamental de cada sistema $\alpha+$ caroço. Os raios $R$ obtidos para os cinco núcleos estudados são apresentados na Tabela 3.1. O valor de $R$ calculado para o ${ }^{94}$ Mo foi de $5.784 \mathrm{fm}$, enquanto o valor relatado na Ref. [3] para o mesmo núcleo é $R=5.793 \mathrm{fm}$. A pequena diferença de $\approx 0.01 \mathrm{fm}$ entre os dois valores demonstra um alto grau de compatibilidade entre os dois raios, levando-se em conta que o raio citado na Ref. [3] 
Tabela 3.1: Valores aplicados ao parâmetro $R$ do potencial $\alpha+$ caroço nos sistemas estudados. Os outros parâmetros do potencial são mantidos fixos para o ajuste de $R$ (detalhes na Subseção $3.4 .2)$.

\begin{tabular}{ccc}
\hline \hline Núcleo & Sistema & $R(\mathrm{fm})$ \\
\hline${ }^{90} \mathrm{Sr}$ & $\alpha+{ }^{86} \mathrm{Kr}$ & 5.321 \\
${ }^{92} \mathrm{Zr}$ & $\alpha+{ }^{88} \mathrm{Sr}$ & 5.295 \\
${ }^{94} \mathrm{Mo}$ & $\alpha+{ }^{90} \mathrm{Zr}$ & 5.784 \\
${ }^{96} \mathrm{Ru}$ & $\alpha+{ }^{92} \mathrm{Mo}$ & 5.808 \\
${ }^{98} \mathrm{Pd}$ & $\alpha+{ }^{94} \mathrm{Ru}$ & 5.825 \\
\hline \hline
\end{tabular}

foi obtido a partir de um método semi-clássico baseado na regra de quantização de Bohr-Sommerfeld.

Na Seção 4.2, é discutido o comportamento do parâmetro $R$ em relação aos raios nucleares do cluster- $\alpha$ e dos caroços, bem como a influência do número quântico $G$ no mesmo parâmetro.

\subsection{Resultados para as bandas de estado fundamental}

As bandas de estado fundamental calculadas para os cinco sistemas $\alpha+$ caroço são mostradas nas Figuras entre 3.2 e 3.6 em comparação com níveis de energia experimentais. Verifica-se que os níveis calculados fornecem uma boa reprodução geral dos espaçamentos entre os níveis experimentais, levando-se em conta que apenas o parâmetro $R$ é ajustado especificamente para cada núcleo e que os parâmetros $V_{0}, a$ e $b$ possuem valores fixos aplicados com o mesmo grau de eficiência em outras regiões de massa.

Deve-se destacar que o espaçamento entre os níveis $0^{+}$e $2^{+}$é reproduzido de forma mais grosseira nos espectros em geral. Esta característica também é encontrada em outros cálculos da estrutura de cluster- $\alpha$ em núcleos de diferentes regiões de massa e com diferentes potenciais de interação $\alpha+$ caroço $[1,2,3,4,14]$. As bandas de níveis gerados pelas diferentes formas de potencial $\alpha+$ caroço, como WoodsSaxon quadrático [2], folding com interação núcleon-núcleon de alcance zero [1], double folding com interação DDM3Y [4, 36], folding derivado da força HasegawaNagata-Yamamoto [35], ou a própria forma usada neste trabalho (Woods-Saxon simples + Woods-Saxon cúbico) [3], possuem em comum o comportamento quaserotacional onde os espaçamentos entre os primeiros níveis são mais reduzidos do 


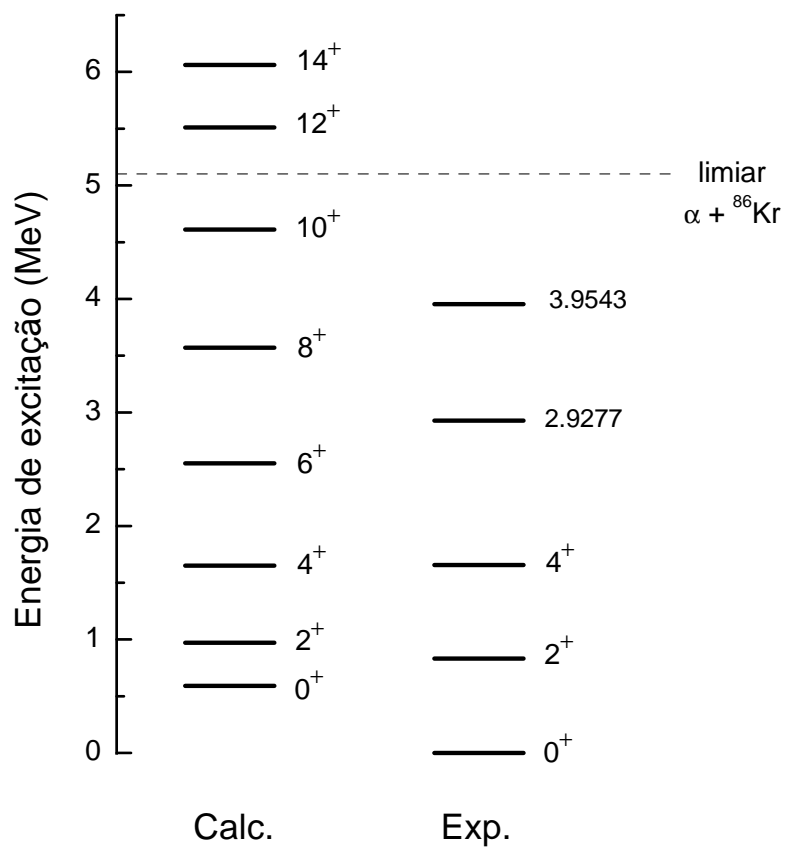

Figura 3.2: Comparação entre a banda de estado fundamental $(G=14)$ calculada para o sistema $\alpha+{ }^{86} \mathrm{Kr}$ e níveis de energia experimentais [37] do ${ }^{90} \mathrm{Sr}$. É sugerido que os níveis experimentais $E_{x}=2.9277 \mathrm{MeV}$ e $E_{x}=3.9543 \mathrm{MeV}$, os quais não possuem spin e paridade identificados, estejam associados aos estados teóricos $6^{+}$e $8^{+}$, respectivamente (ver explicações na Seção 3.6).

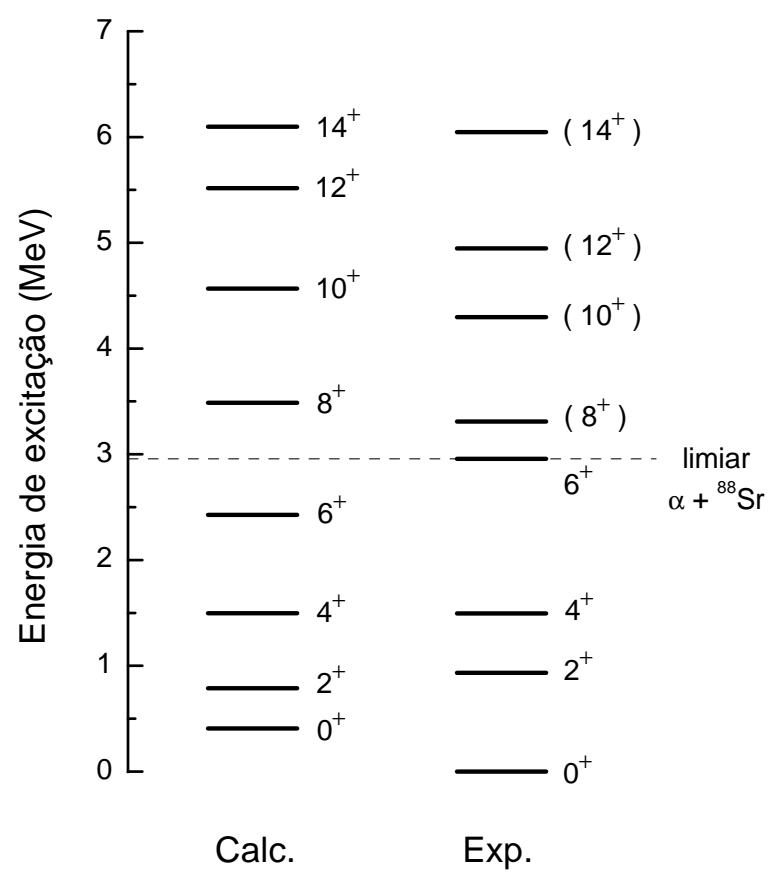

Figura 3.3: Comparação entre a banda de estado fundamental $(G=14)$ calculada para o sistema $\alpha+{ }^{88} \mathrm{Sr}$ e níveis de energia experimentais [38] do ${ }^{92} \mathrm{Zr}$. 


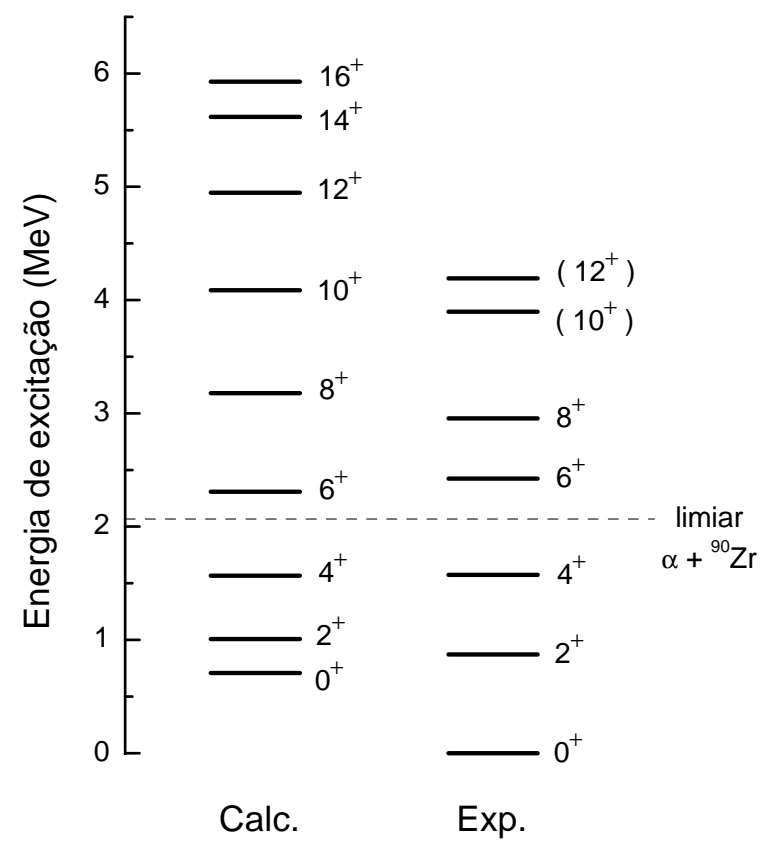

Figura 3.4: Comparação entre a banda de estado fundamental $(G=16)$ calculada para o sistema $\alpha+{ }^{90} \mathrm{Zr}$ e níveis de energia experimentais [40] do ${ }^{94} \mathrm{Mo}$.

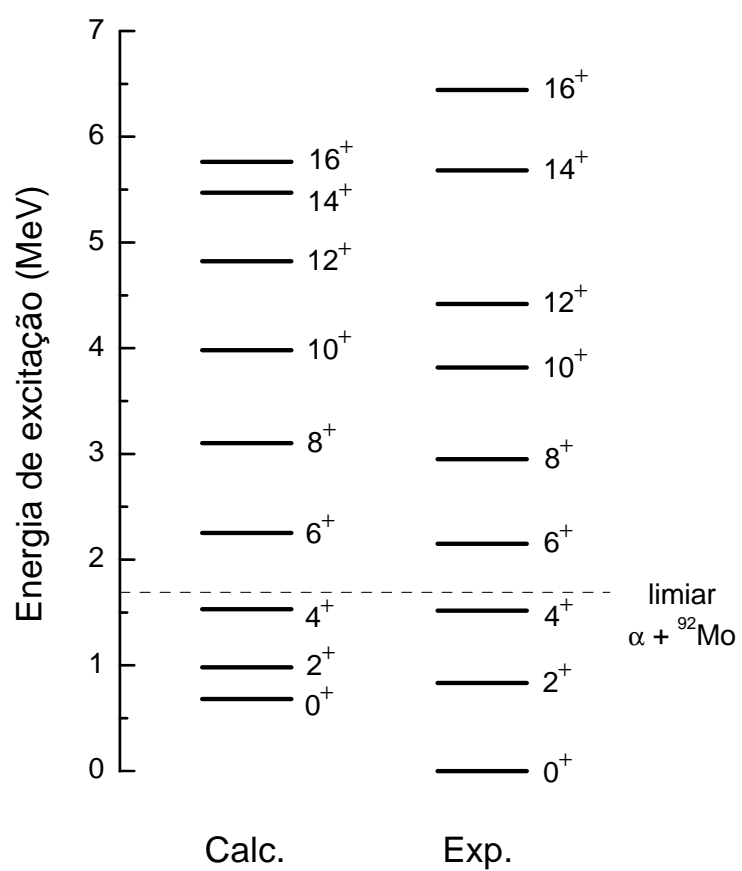

Figura 3.5: Comparação entre a banda de estado fundamental $(G=16)$ calculada para o sistema $\alpha+{ }^{92}$ Mo e níveis de energia experimentais [42] do ${ }^{96} \mathrm{Ru}$. 


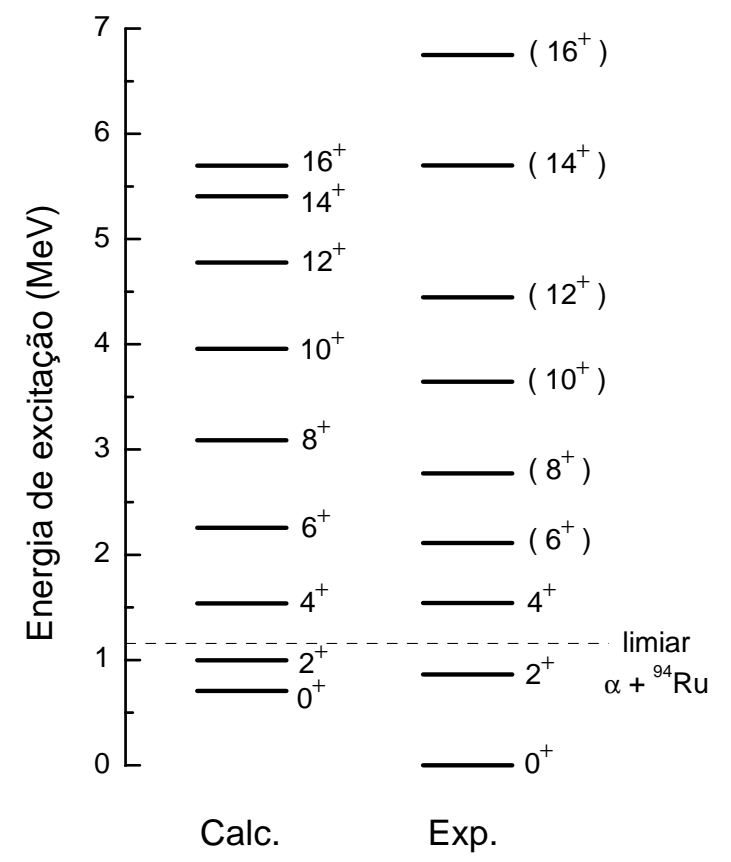

Figura 3.6: Comparação entre a banda de estado fundamental $(G=16)$ calculada para o sistema $\alpha+{ }^{94} \mathrm{Ru}$ e níveis de energia experimentais [43] do ${ }^{98} \mathrm{Pd}$.

que entre os níveis de maior spin. ${ }^{1}$ Vários núcleos analisados neste contexto apresentam espectros experimentais que diferem consideravelmente do comportamento rotacional em seus primeiros níveis, desfavorecendo a reprodução correta do nível $0^{+}$pelo espectro teórico.

O potencial $\alpha+$ caroço usado neste trabalho gera bandas que apresentam aspectos comuns para os cinco núcleos estudados: 1) os primeiros níveis se comportam de forma quase-rotacional; 2) os níveis intermediários possuem espaçamentos de extensão aproximadamente constante; 3) os últimos níveis passam a se comprimir. A característica de compressão nos últimos níveis é notada de forma mais evidente em bandas calculadas com altos spins, tais como na região de massa intermediária e na região dos núcleos pesados. ${ }^{2}$ Este comportamento também resulta numa descrição mais grosseira do espaçamento entre os últimos níveis de algumas bandas experimentais, como ocorre entre os níveis $14^{+}$e $16^{+}$do ${ }^{96} \mathrm{Ru}$ e entre os níveis $\left(14^{+}\right)$e $\left(16^{+}\right)$do ${ }^{98} \mathrm{Pd}$. Apesar do espaçamento entre os níveis experimen-

\footnotetext{
${ }^{1} \mathrm{O}$ potencial do tipo W.Saxon $+\mathrm{W}$.Saxon ${ }^{3}$ gera bandas de comportamento quase-rotacional desde o primeiro nível da banda (band head) até os primeiros níveis intermediários. As outras formas de potencial citadas geram bandas que mantêm um comportamento quase-rotacional mesmo para os estados de spins mais altos.

${ }^{2}$ No caso dos núcleos pesados, o potencial $\alpha+$ caroço do tipo W.Saxon + W.Saxon ${ }^{3}$ pode gerar bandas com alto grau de compressão nos níveis de maior spin, podendo haver inclusive um comportamento de inversão da ordem crescente de spin nos últimos níveis da banda (na Ref. [3], este aspecto é discutido a respeito do ${ }^{212} \mathrm{Po}$ ).
} 
tais $\left(12^{+}\right)$e $\left(14^{+}\right)$do ${ }^{92} \mathrm{Zr}$ ser $\approx 1.9 \times$ maior do que o espaçamento entre seus correspondentes teóricos, o espectro calculado descreve a posição do nível $\left(14^{+}\right)$ de forma satisfatória.

Outra forma de avaliar o grau de eficiência do potencial $\alpha+$ caroço na reprodução dos espectros experimentais é verificando a variação relativa do parâmetro $V_{0}$ para a reprodução correta dos níveis experimentais, como é discutido na Seção 4.1 .

\subsection{Aspectos referentes aos níveis de energia experimentais}

É importante discutir o critério de escolha dos níveis experimentais usados nas comparações com os espectros teóricos. Para os cinco núcleos estudados, foram escolhidos os níveis experimentais $0^{+}, 2^{+}, 4^{+}, \ldots, G^{+}$de energia mais baixa possível, contudo, levando em conta as transições $E 2$ de maior intensidade. Também foi considerado desejável a escolha de níveis populados em reações que indicam ou podem indicar a presença da estrutura de cluster- $\alpha$ nos mesmos níveis. A seguir, são discutidos os detalhes referentes às reações onde os níveis escolhidos são populados, além de outros aspectos experimentais.

Com relação aos níveis experimentais do ${ }^{90} \mathrm{Sr}$, verifica-se que não há correspondentes para os níveis calculados entre $6^{+}$e $14^{+}$[37]. Porém, sugere-se que os níveis experimentais $E_{x}=2.9277 \mathrm{MeV}$ e $E_{x}=3.9543 \mathrm{MeV}$ podem estar associados aos estados teóricos $6^{+}$e $8^{+}$, respectivamente. É constatado experimentalmente que o nível $E_{x}=2.9277 \mathrm{MeV}$ sofre uma transição para o nível $4^{+}\left(E_{x}=1.65591 \mathrm{MeV}\right)$, sendo esta a transição de maior intensidade- $\gamma$ a partir de $E_{x}=2.9277 \mathrm{MeV}$. A respeito do nível $E_{x}=3.9543 \mathrm{MeV}$, é constatado experimentalmente que o mesmo sofre uma transição para o nível $E_{x}=2.9277 \mathrm{MeV}$, não sendo esta a transição de maior intensidade- $\gamma$ a partir de $E_{x}=3.9543 \mathrm{MeV}$. Adicionalmente, o fato dos níveis $E_{x}=2.9277 \mathrm{MeV}$ e $E_{x}=3.9543 \mathrm{MeV}$ estarem próximos dos níveis teóricos $6^{+}$e $8^{+}$motivou esta sugestão de correspondência. No entanto, somente novos dados experimentais a respeito de $E_{x}=2.9277 \mathrm{MeV}$ e $E_{x}=3.9543 \mathrm{MeV}$ podem comprovar se esta correspondência é correta. A Ref. [37] não mostra indicações de que os níveis experimentais do ${ }^{90} \mathrm{Sr}$ associados à banda $G=14$ sejam populados por reações de transferência- $\alpha$, decaimento- $\alpha$ ou outra reação indicativa da estrutura de cluster- $\alpha$.

Discutindo os níveis experimentais do ${ }^{92} \mathrm{Zr}$, verifica-se que há correspondentes para todos os níveis calculados, contudo, alguns níveis experimentais possuem spins e paridades não confirmados. As referências [38] e [39] afirmam que, com exceção do estado $\left(14^{+}\right)$, todos os níveis da banda experimental do ${ }^{92} \mathrm{Zr}$ são popu- 
lados na reação ${ }^{88} \mathrm{Sr}\left({ }^{7} \mathrm{Li}, 2 n p \gamma\right){ }^{92} \mathrm{Zr}$, a qual configura uma forma de transferência- $\alpha$ no ${ }^{88} \mathrm{Sr}$. Este fato pode ser interpretado como uma indicação da estrutura de cluster- $\alpha$ nestes estados, apesar da necessidade de dados de outras experiências de transferência- $\alpha$ para uma confirmação destas indicações. É interessante notar que não há outros níveis de paridade positiva no espectro geral do ${ }^{92} \mathrm{Zr}$ [38] que sejam populados pela reação ${ }^{88} \mathrm{Sr}\left({ }^{7} \mathrm{Li}, 2 n p \gamma\right)$, mostrando que os estados experimentais escolhidos para a comparação com a banda teórica $G=14$ apresentam uma característica específica que os diferencia dos demais estados do ${ }^{92} \mathrm{Zr}$.

Com relação aos níveis experimentais do ${ }^{94} \mathrm{Mo}$, verifica-se que não há correspondentes para os níveis calculados $14^{+}$e $16^{+}$. O espectro geral do ${ }^{94} \mathrm{Mo}$ [40] apresenta alguns níveis $\left(14^{+}\right)$e $\left(16^{+}\right)$acima de $E_{x} \approx 7.5 \mathrm{MeV}$, no entanto, não há transições observadas entre tais níveis e os estados inferiores da banda experimental. O nível experimental $\left(12^{+}\right)\left(E_{x}=4.1915 \mathrm{MeV}\right)$ não é mencionado em outros cálculos referentes à estrutura de cluster- $\alpha$ no ${ }^{94} \mathrm{Mo}[3,4,5]$, contudo, o referido nível foi incluído na banda experimental do ${ }^{94} \mathrm{Mo}$, pois verifica-se na Ref. [40] que há uma transição entre os estados $\left(12^{+}\right)\left(E_{x}=4.1915 \mathrm{MeV}\right)$ e $\left(10^{+}\right)\left(E_{x}=3.8971\right.$ $\mathrm{MeV}$ ) que é possivelmente do tipo $E 2$. As referências [40] e [41] afirmam que os níveis experimentais entre $0^{+}$e $6^{+}$são populados na reação ${ }^{90} \mathrm{Zr}\left({ }^{16} \mathrm{O},{ }^{12} \mathrm{C} \gamma\right){ }^{94} \mathrm{Mo}$, a qual configura uma forma de transferência- $\alpha$ no ${ }^{90} \mathrm{Zr}$. Este fato sugere a presença da estrutura de cluster- $\alpha$ nestes estados. Deve-se levar em conta que, além dos níveis citados anteriormente, outros estados de paridade positiva do ${ }^{94} \mathrm{Mo} \mathrm{são}$ populados na reação ${ }^{90} \mathrm{Zr}\left({ }^{16} \mathrm{O},{ }^{12} \mathrm{C} \gamma\right){ }^{94} \mathrm{Mo}$, mostrando que há uma maior complexidade para a interpretação dos dados referentes a esta reação. A Ref. [41] sugere que os estados da banda experimental possuem a configuração $\left(\nu g_{7 / 2}\right)^{2}\left(\pi g_{9 / 2}\right)^{2}$, o que é compatível com o número quântico $G=16$ adotado para esta banda.

Discutindo os níveis experimentais do ${ }^{96} \mathrm{Ru}$, verifica-se que há correspondentes para todos os níveis calculados. Diferentemente dos núcleos ${ }^{92} \mathrm{Zr} \mathrm{e}{ }^{94} \mathrm{Mo}$, o espectro geral do ${ }^{96} \mathrm{Ru}[42]$ não apresenta dados referentes a reações de transferência- $\alpha$. A Ref. [42] indica que os estados da banda experimental entre $0^{+}$e $6^{+}$são populados no espalhamento inelástico ${ }^{96} \mathrm{Ru}\left(\alpha, \alpha^{\prime}\right)$.

A respeito dos níveis experimentais do ${ }^{98} \mathrm{Pd}$, verifica-se que há correspondentes para todos os níveis calculados, contudo, somente os níveis experimentais $0^{+}, 2^{+}$ e $4^{+}$possuem spins e paridades confirmados. O espectro geral do ${ }^{98} \mathrm{Pd}$ [43] não apresenta dados referentes a reações de transferência- $\alpha$, decaimento- $\alpha$ ou outra reação diretamente relacionada a este contexto.

\subsection{Normalização das funções radiais $u(r)$}

As funções $u(r)$ associadas aos auto-valores de energia (ver eq. (3.8)) dão origem às auto-funções radiais $u_{N, L}(r)$, as quais compõem as auto-soluções $\psi_{N, L, M}(r, \theta, \phi)$ 
da equação de Schrödinger independente do tempo. Para isso, a normalização das funções $u(r)$ torna-se necessária. Inicialmente, deve-se integrar $u^{2}(r)$ sobre todos os $r$ para a obtenção da quantidade

$$
C=\int_{0}^{\infty} u^{2}(r) d r
$$

Assim, a função normalizada $u_{N, L}(r)$ se relaciona com $u(r)$ através da equação

$$
u_{N, L}(r)=\frac{1}{\sqrt{C}} u(r) .
$$

A integração em (3.10) foi feita numericamente pela regra do trapézio composta (detalhes na Ref. [31]). O mesmo método é empregado em outros cálculos deste trabalho onde a integração numérica é necessária. Na prática, a integração numérica foi executada entre 0 e $50 \mathrm{fm}$, visto que a amplitude de $u(r)$ além deste intervalo é desprezível no cálculo de $C$. As funções normalizadas $u_{N, L}(r)$ foram calculadas com passo constante de $0.01 \mathrm{fm}$, visando simplificar o cálculo posterior de outras propriedades dos sistemas $\alpha+$ caroço através das mesmas funções. Devese destacar que os cálculos com passo variável do método RKF foram realizados apenas para a determinação dos auto-valores de energia dos sistemas $\alpha+$ caroço.

\subsection{Funções radiais normalizadas $u_{N, L}(r)$}

Exemplos de funções radiais $u_{N, L}(r)$ calculadas pelos métodos descritos nas Seções 3.2, 3.3 e 3.7 são mostradas nas Figuras 3.7, 3.8 e 3.9, as quais se referem às bandas de estado fundamental dos núcleos ${ }^{92} \mathrm{Zr}$ e ${ }^{96} \mathrm{Ru}$. Especificamente no cálculo das funções $u_{N, L}(r)$, o parâmetro $V_{0}$ do potencial de interação $\alpha+$ caroço foi reajustado para a reprodução correta de cada nível de energia experimental associado a uma das funções radiais. A variação da profundidade $V_{0}$ é necessária neste caso, pois outras propriedades do sistema $\alpha+$ caroço são calculadas através das funções $u_{N, L}(r)$ e, por isto, as mesmas devem ser compatíveis com as reais energias de excitação do núcleo a ser analisado. Os valores de $V_{0}$ correspondentes aos níveis experimentais são mostrados na Tabela 4.1.

Os estados nucleares associados às funções $u_{N, L}(r)$ são identificados através do número de nós internos $N$, que é obtido diretamente do comportamento das mesmas funções, além do número quântico $L$ do momento angular orbital.

As Figuras 3.7, 3.8 e 3.9 também mostram exemplos de funções radiais $u_{N, L}(r)$ calculadas para os estados quase-ligados das bandas $G=14$ e $G=16$ dos núcleos ${ }^{92} \mathrm{Zr}$ e ${ }^{96} \mathrm{Ru}$, respectivamente. Vê-se que a resolução da equação radial com a aproximação de estado ligado gera funções $u_{N, L}(r)$ que claramente tendem a respeitar as condições de contorno características dos níveis ligados. 

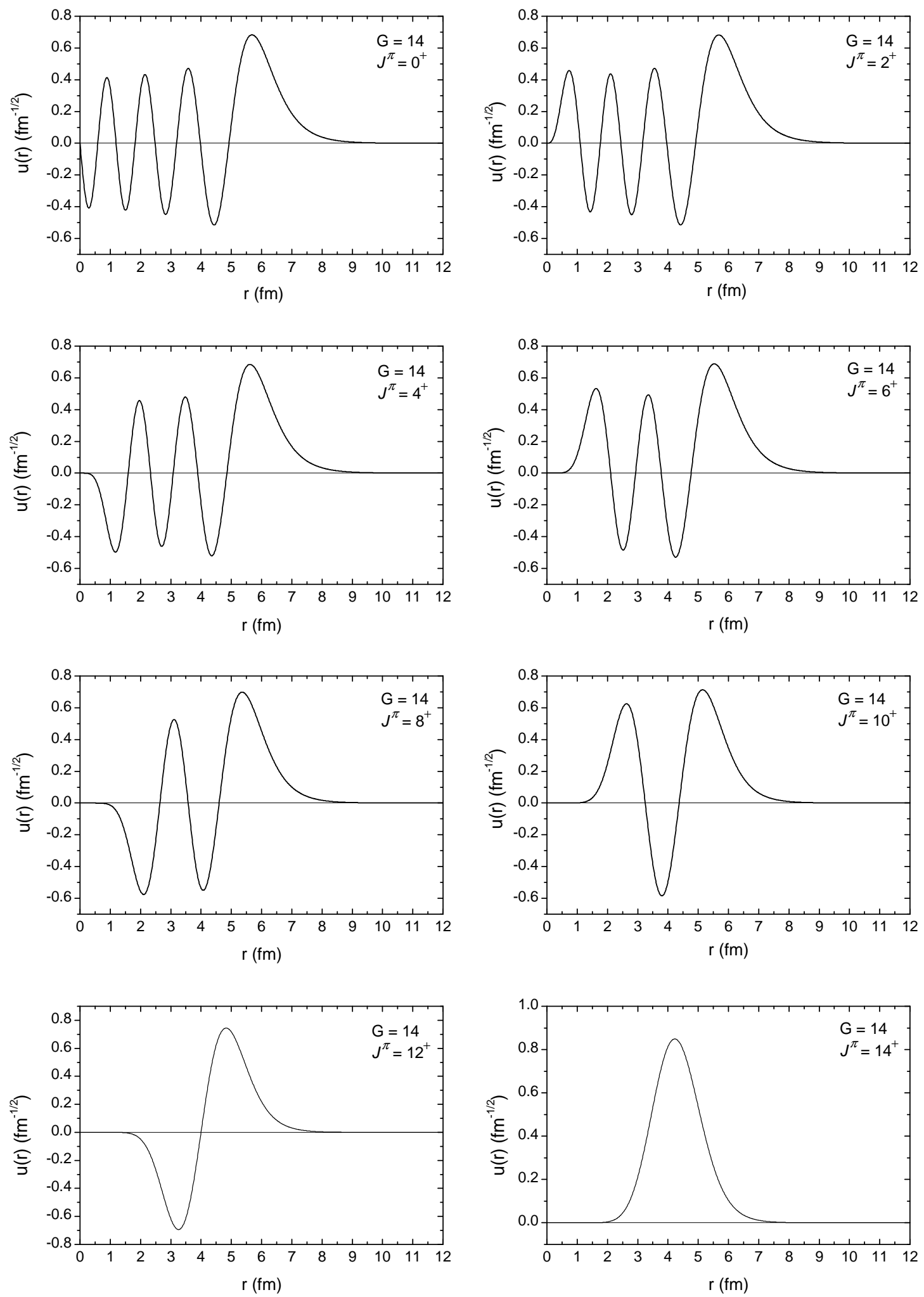

Figura 3.7: Funções de onda radiais $u_{N, L}(r)$ associadas à banda de estado fundamental $(G=14)$ do ${ }^{92} \mathrm{Zr}$. Os estados $J^{\pi}$ correspondentes às funções são indicados. No cálculo das funções, o parâmetro $V_{0}$ do potencial $\alpha+$ caroço foi reajustado para a reprodução correta dos níveis de energia experimentais. 

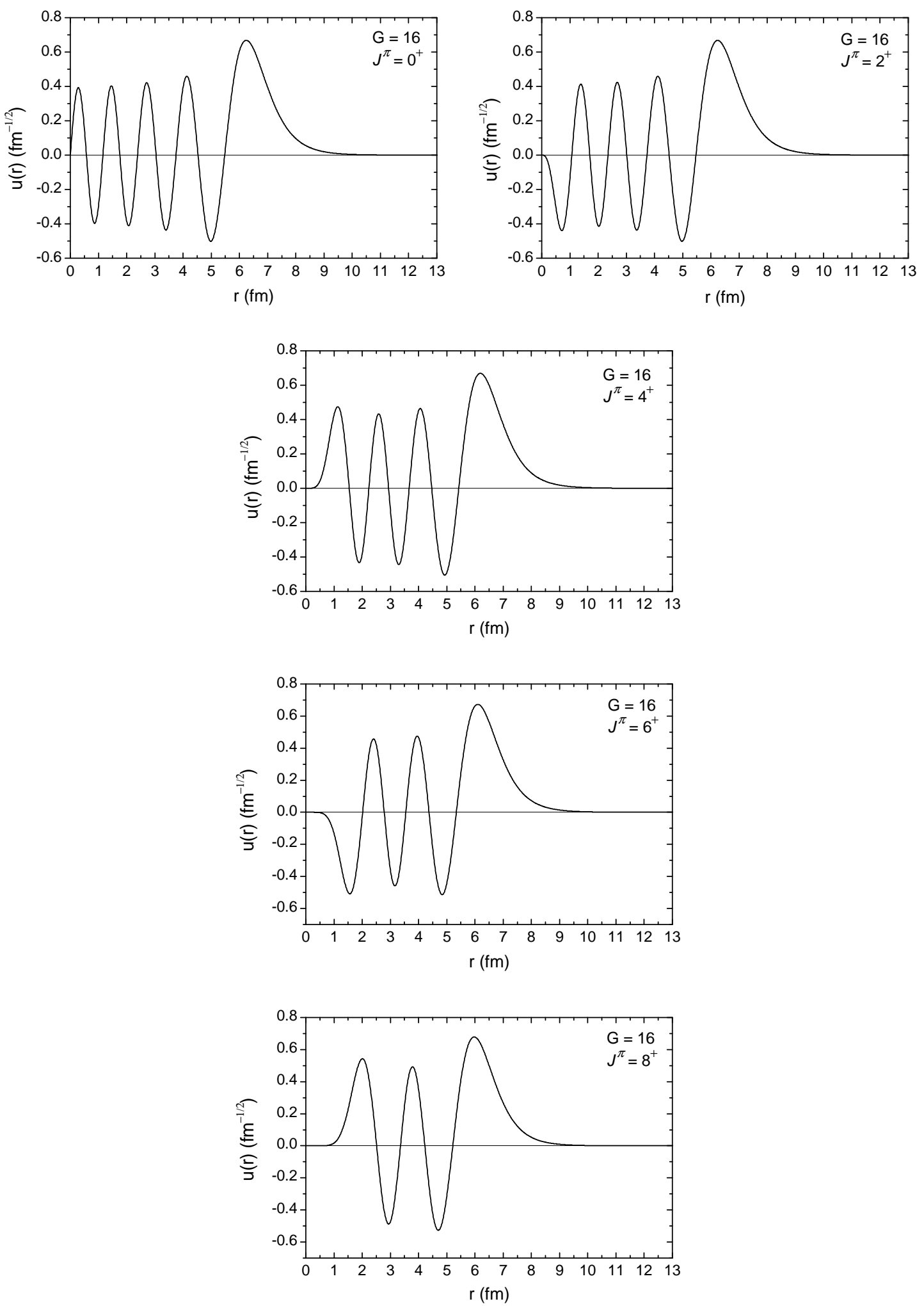

Figura 3.8: Funções de onda radiais $u_{N, L}(r)$ associadas aos estados $0^{+}, 2^{+}, 4^{+}, 6^{+}$e $8^{+}$da banda de estado fundamental $(G=16)$ do ${ }^{96} \mathrm{Ru}$. Os estados $J^{\pi}$ correspondentes às funções são indicados. No cálculo das funções, o parâmetro $V_{0}$ do potencial $\alpha+$ caroço foi reajustado para a reprodução correta dos níveis de energia experimentais. 

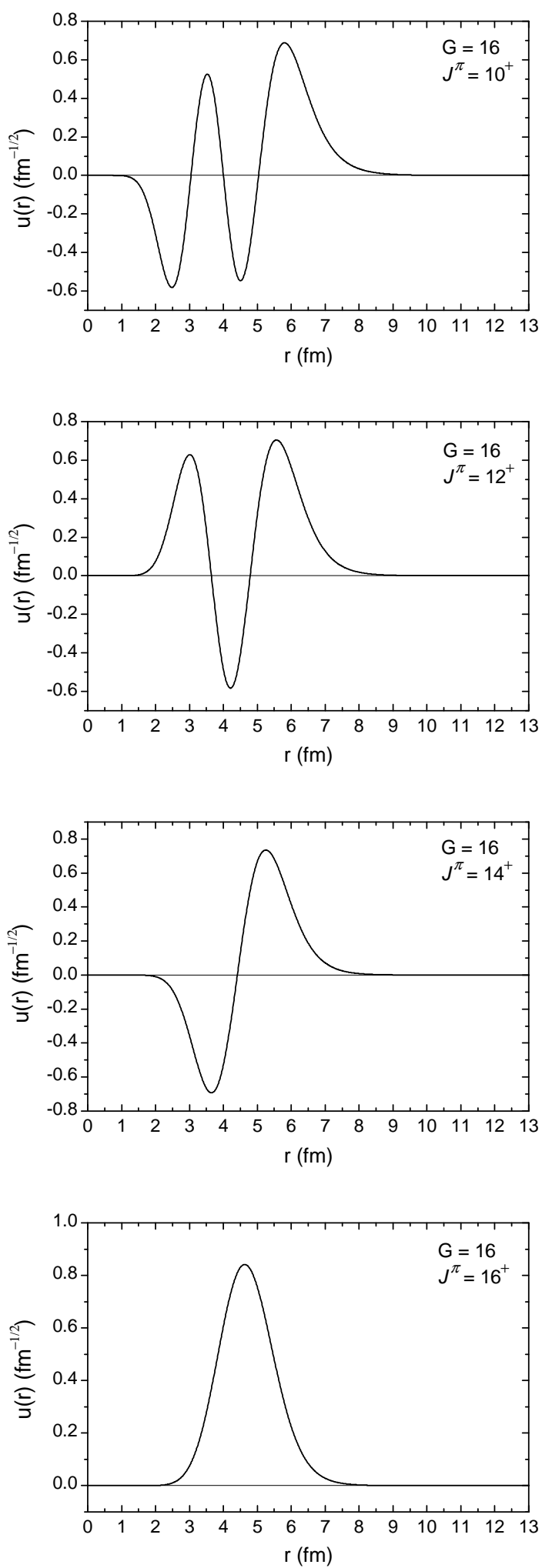

Figura 3.9: Funções de onda radiais $u_{N, L}(r)$ associadas aos estados $10^{+}, 12^{+}, 14^{+}$e $16^{+}$da banda de estado fundamental $(G=16)$ do ${ }^{96} \mathrm{Ru}$. Os estados $J^{\pi}$ correspondentes às funções são indicados. No cálculo das funções, o parâmetro $V_{0}$ do potencial $\alpha+$ caroço foi reajustado para a reprodução correta dos níveis de energia experimentais. 


\section{Observação sobre a implementação dos métodos numéricos}

Os métodos de cálculo numérico descritos neste capítulo, assim como todos os cálculos envolvendo os níveis de energia e funções de onda dos sistemas $\alpha+$ caroço, foram executados através de programas computacionais desenvolvidos na linguagem FORTRAN 77 especificamente para este projeto. A mesma observação é válida para os capítulos seguintes deste trabalho, exceto quando é citado explicitamente outro programa computacional. 


\section{Capítulo 4}

\section{Propriedades das bandas de estado fundamental dos sistemas $\alpha+$ caroço}

Neste capítulo, analisa-se as propriedades das bandas de estado fundamental dos núcleos ${ }^{90} \mathrm{Sr},{ }^{92} \mathrm{Zr},{ }^{94} \mathrm{Mo},{ }^{96} \mathrm{Ru}$ e ${ }^{98} \mathrm{Pd}$. São discutidos o grau de estabilidade do parâmetro $V_{0}$ do potencial $\alpha+$ caroço e o seu comportamento em relação ao número quântico $L$, o comportamento do parâmetro $R$ em relação aos raios dos núcleos envolvidos no sistema, as separações intercluster rms, as larguras- $\alpha$ reduzidas e as taxas de transição $B(E 2)$. Visando uma discussão mais abrangente das propriedades citadas, os núcleos ${ }^{20} \mathrm{Ne}$, ${ }^{44} \mathrm{Ti}$ e ${ }^{212} \mathrm{Po}$ também são analisados em diferentes seções.

\subsection{Comportamento do parâmetro $V_{0}$ do potencial $\alpha+$ caroço}

\subsubsection{Variação de $V_{0}$ em relação ao valor padrão de $220 \mathrm{MeV}$}

A descrição das bandas de estado fundamental dos cinco núcleos estudados foi executada com os parâmetros $V_{0}$, $a$ e $b$ fixos e o parâmetro $R$ variável, como já foi discutido na Subseção 3.4.2. Uma forma de avaliar o grau de eficiência do potencial $\alpha+$ caroço consiste em verificar qual é a variação necessária de $V_{0}, a$ ou $b$ para a reprodução correta dos níveis de energia experimentais. Evitando alterações na difusividade $a$ e no parâmetro $b$, já que ambos afetam diretamente a forma geométrica da parte nuclear do potencial, o coeficiente de profundidade $V_{0}$ foi escolhido para esta avaliação. 
O parâmetro $V_{0}$ foi reajustado para reproduzir cada nível experimental das bandas de estado fundamental com um erro $\leq 5 \mathrm{keV}$. Os valores de $R$ específicos para cada núcleo (Tabela 3.1) foram mantidos neste processo. Os valores de $V_{0}$ correspondentes a cada nível são apresentados na Tabela 4.1. Na mesma tabela, é mostrada a variação de $V_{0}$ relativa ao valor de $220 \mathrm{MeV}$, o qual foi empregado na descrição geral das bandas de estado fundamental dos cinco núcleos estudados. Verifica-se que a variação relativa, dada por

$$
\left(\Delta V_{0}\right)_{\mathrm{rel}}=\frac{V_{0}-220 \mathrm{MeV}}{220 \mathrm{MeV}}
$$

é muito pequena para todos os valores de $V_{0}$ reajustados, sendo que o maior valor registrado em módulo $\left(\left|\left(\Delta V_{0}\right)_{\text {rel }}\right|=1.091 \%\right.$ para o estado $\left(16^{+}\right)$do $\left.{ }^{98} \mathrm{Pd}\right)$ ainda é muito próximo de $1 \%$. Entre os estados com spin e paridade bem estabelecidos na literarura, o maior valor registrado em módulo refere-se ao estado $0^{+}$do ${ }^{94} \mathrm{Mo}$, estando abaixo de $1 \%$ de variação relativa. Portanto, os números apresentados na Tabela 4.1 mostram que o valor fixo $V_{0}=220 \mathrm{MeV}$ é bastante apropriado para uma descrição geral dos espectros dos cinco núcleos, juntamente com os valores fixos $a=0.65 \mathrm{fm}$ e $b=0.3$. Adicionalmente, constata-se que o grau de eficiência dos valores fixos de $V_{0}, a$ e $b$ na região do Mo é próximo daquele observado nas bandas de estado fundamental dos núcleos ${ }^{20} \mathrm{Ne}$, ${ }^{44} \mathrm{Ti}$ e ${ }^{212} \mathrm{Po}$ [3], os quais pertencem a regiões de massa distintas.

\subsubsection{Comportamento do parâmetro $V_{0}$ em relação ao número quântico $L$}

Possuindo os valores de $V_{0}$ reajustados para a reprodução dos níveis experimentais, pode-se observar como este parâmetro varia em relação ao número do momento angular orbital $L$. Com isto, permite-se revelar se o parâmetro $V_{0}$ apresenta um comportamento comum para os cinco núcleos estudados.

A Figura 4.1 mostra os valores de $V_{0}$ calculados para os cinco núcleos da região do Mo em relação a $L$. Nota-se que $V_{0}$ varia de forma semelhante para os cinco núcleos, apesar de algumas diferenças locais mais significativas. No intervalo entre $L=0$ e $L=6$, há um comportamento geral decrescente de $V_{0}$ que se encerra com um mínimo local em $L=4$ para os núcleos ${ }^{96} \mathrm{Ru}$ e ${ }^{98} \mathrm{Pd}$ e um mínimo local em $L=6$ para os núcleos ${ }^{92} \mathrm{Zr}$ e ${ }^{94} \mathrm{Mo}$. É sugerido que $V_{0}$ também apresenta um mínimo local em $L=6$ para o ${ }^{90} \mathrm{Sr}$, contudo, não é possível confirmar este fato, pois não há níveis $6^{+}$e $8^{+}$identificados experimentalmente para este núcleo (detalhes na Seção 3.6). Com exceção do ${ }^{90} \mathrm{Sr}$, nota-se que $V_{0}$ apresenta um comportamento geral crescente a partir do mínimo local em $L=4$ ou 6 , continuando até um ponto máximo local em $L=12$. Apesar dos núcleos ${ }^{92} \mathrm{Zr},{ }^{94} \mathrm{Mo},{ }^{96} \mathrm{Ru}$ e ${ }^{98} \mathrm{Pd}$ 
Tabela 4.1: Valores do parâmetro $V_{0}$ reajustados para a reprodução correta dos níveis de energia experimentais das bandas de estado fundamental. São indicados os estados $J^{\pi}$ com as energias de excitação $E_{x}$ correspondentes e a variação $\left(\Delta V_{0}\right)_{\text {rel }}$ relativa ao valor padrão de $220 \mathrm{MeV}$. Os estados acompanhados de (?) não possuem spin e paridade identificados experimentalmente, sendo sugerida uma associação com os estados teóricos mencionados (detalhes na Seção 3.6). Os estados entre parênteses possuem spins e paridades sem confirmação definitiva na literatura.

\begin{tabular}{|c|c|c|c|}
\hline \multicolumn{4}{|c|}{ Núcleo: ${ }^{90} \mathrm{Sr}$} \\
\hline$J^{\pi}$ & $E_{x}(\mathrm{MeV})$ & $V_{0}(\mathrm{MeV})$ & $\left(\Delta V_{0}\right)_{\text {rel }}(\%)$ \\
\hline $0^{+}$ & 0.000 & 221.50 & 0.682 \\
\hline $2^{+}$ & 0.832 & 220.36 & 0.164 \\
\hline $4^{+}$ & 1.656 & 220.01 & 0.005 \\
\hline $6^{+}(?)$ & 2.928 & 219.04 & -0.436 \\
\hline $8^{+}(?)$ & 3.954 & 219.06 & -0.427 \\
\hline \multicolumn{4}{|c|}{ Núcleo: ${ }^{92} \mathrm{Zr}$} \\
\hline$J^{\pi}$ & $E_{x}(\mathrm{MeV})$ & $V_{0}(\mathrm{MeV})$ & $\left(\Delta V_{0}\right)_{\text {rel }}(\%)$ \\
\hline $0^{+}$ & 0.000 & 221.06 & 0.482 \\
\hline $2^{+}$ & 0.934 & 219.62 & -0.173 \\
\hline $4^{+}$ & 1.495 & 220.00 & 0.000 \\
\hline $6^{+}$ & 2.958 & 218.64 & -0.618 \\
\hline$\left(8^{+}\right)$ & 3.309 & 220.45 & 0.205 \\
\hline$\left(10^{+}\right)$ & 4.297 & 220.67 & 0.305 \\
\hline$\left(12^{+}\right)$ & 4.948 & 221.37 & 0.623 \\
\hline$\left(14^{+}\right)$ & 6.046 & 220.11 & 0.050 \\
\hline \multicolumn{4}{|c|}{ Núcleo: ${ }^{94} \mathrm{Mo}$} \\
\hline$J^{\pi}$ & $E_{x}(\mathrm{MeV})$ & $V_{0}(\mathrm{MeV})$ & $\left(\Delta V_{0}\right)_{\mathrm{rel}}(\%)$ \\
\hline $0^{+}$ & 0.000 & 221.80 & 0.818 \\
\hline $2^{+}$ & 0.871 & 220.37 & 0.168 \\
\hline $4^{+}$ & 1.574 & 219.98 & -0.009 \\
\hline $6^{+}$ & 2.423 & 219.70 & -0.136 \\
\hline $8^{+}$ & 2.956 & 220.54 & 0.245 \\
\hline$\left(10^{+}\right)$ & 3.897 & 220.46 & 0.209 \\
\hline$\left(12^{+}\right)$ & 4.192 & 221.78 & 0.809 \\
\hline
\end{tabular}


Tabela 4.1 (continuação)

\begin{tabular}{cccc}
\hline \hline & \multicolumn{3}{c}{ Núcleo: ${ }^{96} \mathrm{Ru}$} \\
$J^{\pi}$ & $E_{x}(\mathrm{MeV})$ & $V_{0}(\mathrm{MeV})$ & $\left(\Delta V_{0}\right)_{\text {rel }}(\%)$ \\
\hline $0^{+}$ & 0.000 & 221.72 & 0.782 \\
$2^{+}$ & 0.833 & 220.38 & 0.173 \\
$4^{+}$ & 1.518 & 220.02 & 0.009 \\
$6^{+}$ & 2.150 & 220.25 & 0.114 \\
$8^{+}$ & 2.950 & 220.36 & 0.164 \\
$10^{+}$ & 3.817 & 220.38 & 0.173 \\
$12^{+}$ & 4.418 & 220.92 & 0.418 \\
$14^{+}$ & 5.681 & 219.50 & -0.227 \\
$16^{+}$ & 6.442 & 218.44 & -0.709 \\
& & & \\
& & Núcleo: ${ }^{98} \mathrm{Pd}$ & $\left(\Delta V_{0}\right)_{\text {rel }}(\%)$ \\
$J^{\pi}$ & $E_{x}(\mathrm{MeV})$ & $V_{0}(\mathrm{MeV})$ & 0.809 \\
$0^{+}$ & 0.000 & 221.78 & 0.145 \\
$2^{+}$ & 0.863 & 220.32 & 0.000 \\
$4^{+}$ & 1.541 & 220.00 & 0.155 \\
$\left(6^{+}\right)$ & 2.112 & 220.34 & 0.336 \\
$\left(8^{+}\right)$ & 2.773 & 220.74 & 0.336 \\
$\left(10^{+}\right)$ & 3.645 & 220.74 & 0.345 \\
$\left(12^{+}\right)$ & 4.447 & 220.76 & -1.091 \\
$\left(14^{+}\right)$ & 5.699 & 219.34 & 217.60 \\
$\left(16^{+}\right)$ & 6.749 & & \\
\hline \hline
\end{tabular}

possuirem em comum o máximo local em $L=12$, há uma variação de $\approx 1 \mathrm{MeV}$ entre os valores de $V_{0}$ situados neste valor de $L$. Para $L>12$, onde há somente dados referentes aos núcleos ${ }^{92} \mathrm{Zr},{ }^{96} \mathrm{Ru}$ e ${ }^{98} \mathrm{Pd}$, o parâmetro $V_{0}$ volta a ter um comportamento decrescente.

A semelhança de comportamento da profundidade $V_{0}$ para os diferentes núcleos é favorável para a escolha de funções que possam descrever, mesmo com algumas discrepâncias, a variação de $V_{0}$ em função de $L$. Neste trabalho, dois tipos de função foram escolhidos para este propósito: a função polinomial de terceiro grau

$$
y_{1}(x)=a_{1} x^{3}+b_{1} x^{2}
$$

e a função do tipo 


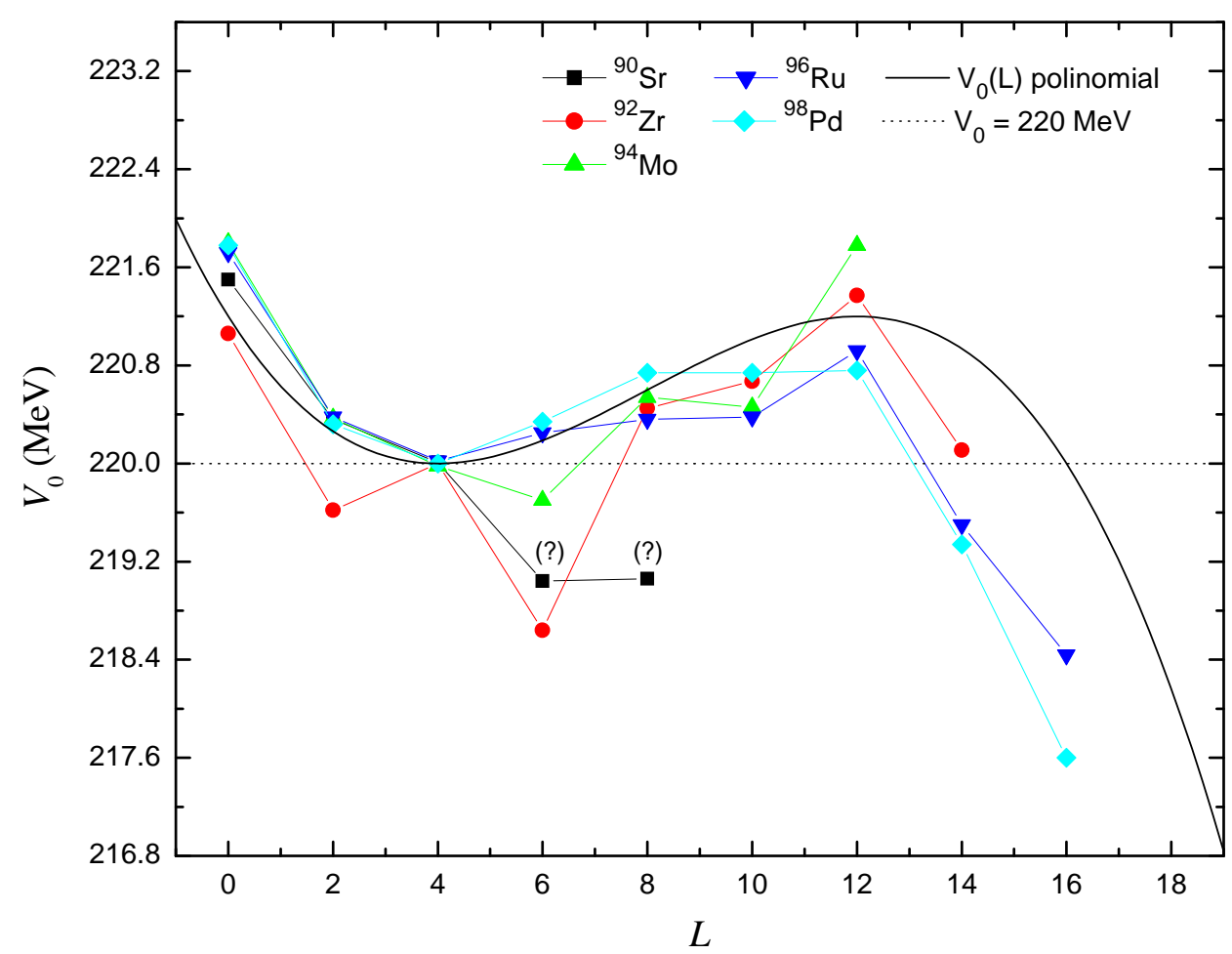

Figura 4.1: Profundidades $V_{0}$ ajustadas para a reprodução dos níveis experimentais das bandas de estado fundamental dos núcleos ${ }^{90} \mathrm{Sr},{ }^{92} \mathrm{Zr},{ }^{94} \mathrm{Mo},{ }^{96} \mathrm{Ru}$ e ${ }^{98} \mathrm{Pd}$ em função do número quântico $L$. A linha preta contínua mostra a função $V_{0}(L)$ na forma polinomial (ver eq. (4.5)) com seus pontos de mínimo e máximo local escolhidos adequadamente para uma descrição satisfatória dos gráficos $V_{0} \times L$. A linha pontilhada indica o valor constante $V_{0}=220 \mathrm{MeV}$ usado para a descrição geral dos espectros dos cinco núcleos. Os pontos acompanhados do símbolo (?) estão relacionados a níveis experimentais com spins e paridades não identificados, sendo sugerida uma associação com os números $L$ correspondentes. (detalhes na Seção 3.6).

$$
y_{2}(x)=\frac{\exp \left(a_{2} x\right)}{\exp \left(b_{2} x^{3}\right)}
$$

onde $a_{1}, b_{1}, a_{2}$ e $b_{2}$ são constantes. Para que tais funções sejam usadas na descrição do comportamento de $V_{0}$, devem ser feitas adaptações que envolvem mudanças de coordenadas e a determinação das constantes $a_{1}, b_{1}, a_{2}$ e $b_{2}$ através dos dados referentes aos pontos $\left(L, V_{0}\right)$. Neste trabalho, evitou-se o uso de métodos como interpolação e ajuste de mínimos quadrados, pois é desejado que a descrição dos gráficos $V_{0} \times L$ seja feita por um processo simples e aplicável aos cinco núcleos simultaneamente. A escolha de funções com apenas 2 parâmetros também visa simplificar ao máximo a sua adaptação ao comportamento dos gráficos $V_{0} \times L$.

A adaptação das funções em (4.2) e (4.3) é realizada de tal forma que os pontos de máximo e mínimo observados nos gráficos $V_{0} \times L$ sejam reproduzidos de uma forma razoável para o conjunto dos núcleos. Assim, considerando uma função 
$V_{0}(L)$, é estabelecido que

$$
\left(L_{\min }, V_{0}^{\min }\right)=(4,220.0) \quad \text { e } \quad\left(L_{\max }, V_{0}^{\max }\right)=(12,221.2),
$$

onde $\left(L_{\min }, V_{0}^{\min }\right)$ e $\left(L_{\max }, V_{0}^{\max }\right)$ são os pontos de mínimo local e máximo local de $V_{0}(L)$, respectivamente, e $V_{0}^{\min }$ e $V_{0}^{\max }$ são dados em MeV. A escolha de $\left(L_{\min }, V_{0}^{\text {min }}\right)$ é justificada pelas seguintes características: 1$)$ o ponto $(4,220.0)$ é mínimo local dos núcleos ${ }^{96} \mathrm{Ru}$ e ${ }^{98} \mathrm{Pd}$ (considerando uma precisão de décimos de $\mathrm{MeV}$ ); 2) os cinco gráficos $V_{0} \times L$ apresentam pontos em $(4,220.0)$ (considerando uma precisão de décimos de $\mathrm{MeV})$; 3) o ponto de mínimo em $(4,220.0)$ é favorável para a descrição do comportamento decrescente de $V_{0}$ em $L<4$ e do comportamento crescente de $V_{0}$ em $L>4$ para a maioria dos pontos $\left(L, V_{0}\right)$ dos cinco gráficos. Quanto à escolha de $\left(L_{\max }, V_{0}^{\max }\right)$, ela é justificada pelas características: 1) há pontos de máximo local em $L=12$ para os núcleos ${ }^{92} \mathrm{Zr},{ }^{94} \mathrm{Mo},{ }^{96} \mathrm{Ru}$ e ${ }^{98} \mathrm{Pd}$; 2) o valor $V_{0}^{\max }=221.2$ é aproximadamente o valor médio das profundidades $V_{0}$ dos cinco núcleos em $L=12$.

Os pontos $\left(L_{\min }, V_{0}^{\min }\right)$ e $\left(L_{\max }, V_{0}^{\max }\right)$ determinam como se realiza a mudança das coordenadas $x$ e $y_{1}$ na função (4.2) e $x$ e $y_{2}$ na função (4.3) para as coordenadas $L$ e $V_{0}$, também permitindo o cálculo das constantes $a_{1}, b_{1}, a_{2}$ e $b_{2}$ em termos de $L_{\min }, L_{\max }, V_{0}^{\min }$ e $V_{0}^{\max }$. Os detalhes para a adaptação das funções (4.2) e (4.3) às coordenadas $L$ e $V_{0}$ são mostrados no Apêndice A deste trabalho. A função (4.2) adaptada resulta em

$$
V_{0}(L)=a_{1}\left(L-L_{\min }\right)^{3}+b_{1}\left(L-L_{\min }\right)^{2}+V_{0}^{\min }
$$

onde

$$
a_{1}=-\frac{2\left(V_{0}^{\max }-V_{0}^{\min }\right)}{\left(L_{\max }-L_{\min }\right)^{3}} \quad \text { e } \quad b_{1}=\frac{3\left(V_{0}^{\max }-V_{0}^{\min }\right)}{\left(L_{\max }-L_{\min }\right)^{2}} .
$$

No caso da função (4.3), não há um coeficiente externo à expressão $\exp \left(a_{2} x\right) / \exp \left(b_{2} x^{3}\right)$ para que a dimensão de $\mathrm{MeV}$ seja atribuída à função resultante $V_{0}(L)$. Por isto, a função (4.3) foi adaptada inicialmente para as coordenadas adimensionais $L$ e $\lambda$, sendo que $\lambda$ se relaciona com $V_{0}$ por

$$
\lambda=\frac{V_{0}}{V_{0}^{\min }} .
$$

Desta forma, a função resultante $V_{0}(L)$ surge da função adimensional $\lambda(L)$ multiplicada pela constante $V_{0}^{\text {min }}$. Com estes procedimentos, a função (4.3) adaptada resulta em

$$
V_{0}(L)=V_{0}^{\min } \lambda(L)
$$


onde

$$
\begin{gathered}
\lambda(L)=\frac{\exp \left[a_{2}\left(L-L_{\min }-\alpha\right)\right]}{\exp \left[b_{2}\left(L-L_{\min }-\alpha\right)^{3}\right]}+1-\frac{\exp \left(-a_{2} \alpha\right)}{\exp \left(-b_{2} \alpha^{3}\right)}, \\
a_{2}=\frac{3}{L_{\max }-L_{\min }} \operatorname{arcsenh}\left(\frac{\lambda_{\max }-\lambda_{\min }}{2}\right), \\
b_{2}=\frac{4}{\left(L_{\max }-L_{\min }\right)^{3}} \operatorname{arcsenh}\left(\frac{\lambda_{\max }-\lambda_{\min }}{2}\right), \\
\alpha=\frac{1}{2}\left(L_{\max }-L_{\min }\right),
\end{gathered}
$$

e $\lambda_{\min }$ e $\lambda_{\max }$ estão associados respectivamente a $V_{0}^{\min }$ e $V_{0}^{\max }$ conforme a eq. (4.7).

Para que as funções $V_{0}(L)$ sejam comparadas aos gráficos $V_{0} \times L$, deve-se calcular numericamente $a_{1}, b_{1}, a_{2}$ e $b_{2}$ através dos valores propostos em (4.4). Desta forma, obtém-se:

$$
\begin{gathered}
a_{1}=-4.6875 \times 10^{-3} \mathrm{MeV}, \quad b_{1}=0.05625 \mathrm{MeV}, \\
a_{2}=1.0226 \times 10^{-3} \text { e } \quad b_{2}=2.1305 \times 10^{-5} \quad \text { (adimensionais) } .
\end{gathered}
$$

A função proposta $V_{0}(L)$ na forma polinomial é mostrada graficamente na Figura 4.1 em comparação com os pontos $\left(L, V_{0}\right)$ dos cinco núcleos. Foi constatado que as funções $V_{0}(L)$ nas formas polinomial e tipo $\exp \left(a_{2} x\right) / \exp \left(b_{2} x^{3}\right)$ são bastante próximas dentro do intervalo $0 \leq L \leq 20$, de tal maneira que se torna desprezível a diferença entre as duas funções, considerando a escala em que os gráficos são apresentados na Fig. 4.1. As duas funções $V_{0}(L)$ são mostradas em maior escala na Figura 4.2, a qual revela que a diferença entre ambas torna-se significativa somente para valores de $L$ muito acima daqueles presentes nas bandas de estado fundamental dos núcleos estudados. Portanto, a escolha de uma ou outra forma de $V_{0}(L)$ produz a mesma qualidade de descrição dos pontos $\left(L, V_{0}\right)$.

A Figura 4.1 mostra que a função $V_{0}(L)$ oferece uma descrição geral satisfatória para o conjunto de gráficos $V_{0} \times L$. No intervalo $0 \leq L \leq 12$, a função $V_{0}(L)$ fornece uma melhoria da profundidade $V_{0}$ para a maioria dos pontos $\left(L, V_{0}\right)$, se for comparada à profundidade padrão de $220 \mathrm{MeV}$, significando que $V_{0}(L)$ serve como um termo de correção eficiente para o potencial $\alpha+$ caroço dentro deste intervalo. Em particular, os pontos referentes ao ${ }^{92} \mathrm{Zr}$ em $L=2$ e $L=6$ são reproduzidos mais grosseiramente; porém, deve-se considerar que o comportamento dos 2 pontos mencionados difere mais fortemente daquele apresentado pelos outros pontos $\left(L, V_{0}\right)$ em $L=2$ e $L=6$, e que a função $V_{0}(L)$ privilegia os pontos mais próximos de um comportamento comum. 


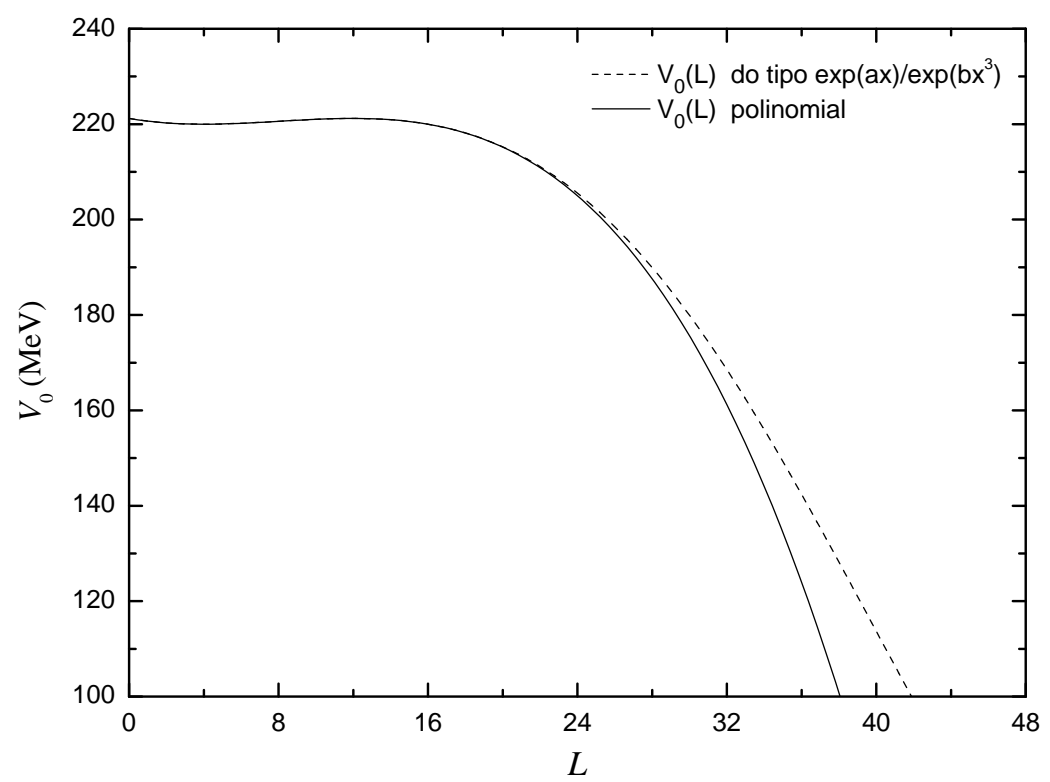

Figura 4.2: Comparação entre as funções $V_{0}(L)$ nas formas polinomial e tipo $\exp \left(a_{2} x\right) / \exp \left(b_{2} x^{3}\right)$, cujos pontos de mínimo e máximo local foram escolhidos adequadamente para uma descrição satisfatória dos gráficos $V_{0} \times L$ (ver Fig. 4.1) dos núcleos ${ }^{90} \mathrm{Sr},{ }^{92} \mathrm{Zr},{ }^{94} \mathrm{Mo},{ }^{96} \mathrm{Ru}$ e ${ }^{98} \mathrm{Pd}$.

Para $L>12$, a função $V_{0}(L)$ fornece uma reprodução mais grosseira dos poucos pontos $\left(L, V_{0}\right)$ desta região; contudo, a mesma função possui o mérito de reproduzir o comportamento geral decrescente dos valores de $V_{0}$ em $L>12$.

\subsubsection{Comportamento do parâmetro $V_{0}$ em outras regiões de massa}

É interessante verificar como o parâmetro $V_{0}$ se comporta em outras regiões de massa e se tal comportamento é semelhante ao observado nos núcleos da região do Mo. Para isso, os sistemas $\alpha+$ caroço correspondentes aos núcleos ${ }^{20} \mathrm{Ne}$ e ${ }^{44} \mathrm{Ti}$ foram analisados pelos mesmos procedimentos descritos no Capítulo 3, e o núcleo ${ }^{212} \mathrm{Po}$ foi analisado por um procedimento semelhante ao adotado no Cap. 3, contudo, usando algumas adaptações necessárias para estados ressonantes (o procedimento para a determinação de níveis ressonantes é discutido na Subseção 5.2.2).

A forma adotada para o potencial $\alpha+$ caroço nos sistemas $\alpha+{ }^{16} \mathrm{O}, \alpha+{ }^{40} \mathrm{Ca}$ e $\alpha+{ }^{208} \mathrm{~Pb}$ é a mesma usada nos núcleos da região do Mo. Seguindo a condição de Wildermuth (ver eq. (1.1)), obtém-se os números quânticos $G$ que caracterizam as bandas de estado fundamental dos sistemas $\alpha+{ }^{16} \mathrm{O}$ e $\alpha+{ }^{40} \mathrm{Ca}$ (ver Tabela 4.2). Para o sistema $\alpha+{ }^{208} \mathrm{~Pb}$, o número $G$ foi obtido através da Ref. [3], onde é mencionado que $G=20$ é o número mais adequado para a descrição dos níveis experimentais do ${ }^{212} \mathrm{Po}$ com os valores fixos já aplicados aos parâmetros $V_{0}, a$ e $b$. O raio $R$ foi ajustado especificamente para cada núcleo, enquanto os parâmetros 
Tabela 4.2: Números quânticos $G$ associados às bandas de estado fundamental dos núcleos ${ }^{20} \mathrm{Ne}$, ${ }^{44} \mathrm{Ti}$ e ${ }^{212} \mathrm{Po}$ e respectivos valores aplicados ao parâmetro $R$ do potencial $\alpha+$ caroço.

\begin{tabular}{cccc}
\hline \hline Núcleo & Sistema & $G$ & $R(\mathrm{fm})$ \\
\hline${ }^{20} \mathrm{Ne}$ & $\alpha+{ }^{16} \mathrm{O}$ & 8 & 3.272 \\
${ }^{44} \mathrm{Ti}$ & $\alpha+{ }^{40} \mathrm{Ca}$ & 12 & 4.551 \\
${ }^{212} \mathrm{Po}$ & $\alpha+{ }^{208} \mathrm{~Pb}$ & 20 & 7.019 \\
\hline \hline
\end{tabular}

$V_{0}, a$ e $b$ foram mantidos com os mesmos valores-padrão. Para a determinação de $R$, é repetido o procedimento no qual este parâmetro é ajustado para reproduzir com o menor erro possível a energia experimental do estado $4^{+}$da banda de estado fundamental. A Tabela 4.2 mostra os valores de $R$ correspondentes aos três sistemas.

Possuindo os raios $R$ adequados aos três núcleos, foi possível reajustar o parâmetro $V_{0}$ para reproduzir cada nível experimental das bandas de estado fundamental (os níveis experimentais foram extraídos das Refs. [44, 45, 46]). Os valores obtidos para $V_{0}$ são apresentados graficamente na Figura 4.3 em função do número quântico $L$. Na mesma figura, são incluídos os valores de $V_{0}$ referentes aos níveis da banda de estado fundamental do ${ }^{94}$ Mo. Assim, é possível analisar simultaneamente o comportamento de $V_{0}$ nos quatro principais núcleos onde a estrutura $\alpha+$ caroço é estudada. Observando a Figura 4.3, nota-se que há maior dificuldade para se encontrar uma função $V_{0}(L)$ que descreva satisfatoriamente o comportamento dos quatro gráficos $V_{0} \times L$, pois alguns pontos $\left(L, V_{0}\right)$ referentes aos núcleos ${ }^{20} \mathrm{Ne}$ e ${ }^{44} \mathrm{Ti}$ se distanciam em muitos $\mathrm{MeV}$ dos pontos referentes ao ${ }^{94} \mathrm{Mo}$ e ${ }^{212} \mathrm{Po}$ com os mesmos valores de $L$. Por outro lado, há um comportamento decrescente que é semelhante para os quatro gráficos $V_{0} \times L$ no intervalo $0 \leq L \leq 4$. Para $L>4$, não se observa um comportamento comum para os quatro núcleos, exceto pelos pontos $\left(L, V_{0}\right)$ referentes aos núcleos ${ }^{94} \mathrm{Mo} \mathrm{e}{ }^{212}$ Po no intervalo $4 \leq L \leq 10$. Infelizmente, não há um nível experimental $12^{+}$conhecido para o ${ }^{212} \mathrm{Po}$, o que evita a verificação de um possível máximo local de $V_{0}$ em $L=12$ para os núcleos ${ }^{44} \mathrm{Ti},{ }^{94} \mathrm{Mo} \mathrm{e}{ }^{212} \mathrm{Po}$.

Apesar da dificuldade mencionada no parágrafo anterior, a função $V_{0}(L)$ na forma polinomial (ver eq. (4.5)) é usada para a descrição dos gráficos $V_{0} \times L$ referentes aos quatro núcleos. É repetido o procedimento da Subseção 4.1.2 onde são definidos os pontos de mínimo local e máximo local da função $V_{0}(L)$. Desta forma, considerando os dados referentes ao ${ }^{20} \mathrm{Ne},{ }^{44} \mathrm{Ti},{ }^{94} \mathrm{Mo}$ e ${ }^{212} \mathrm{Po}$, é estabelecido que: 


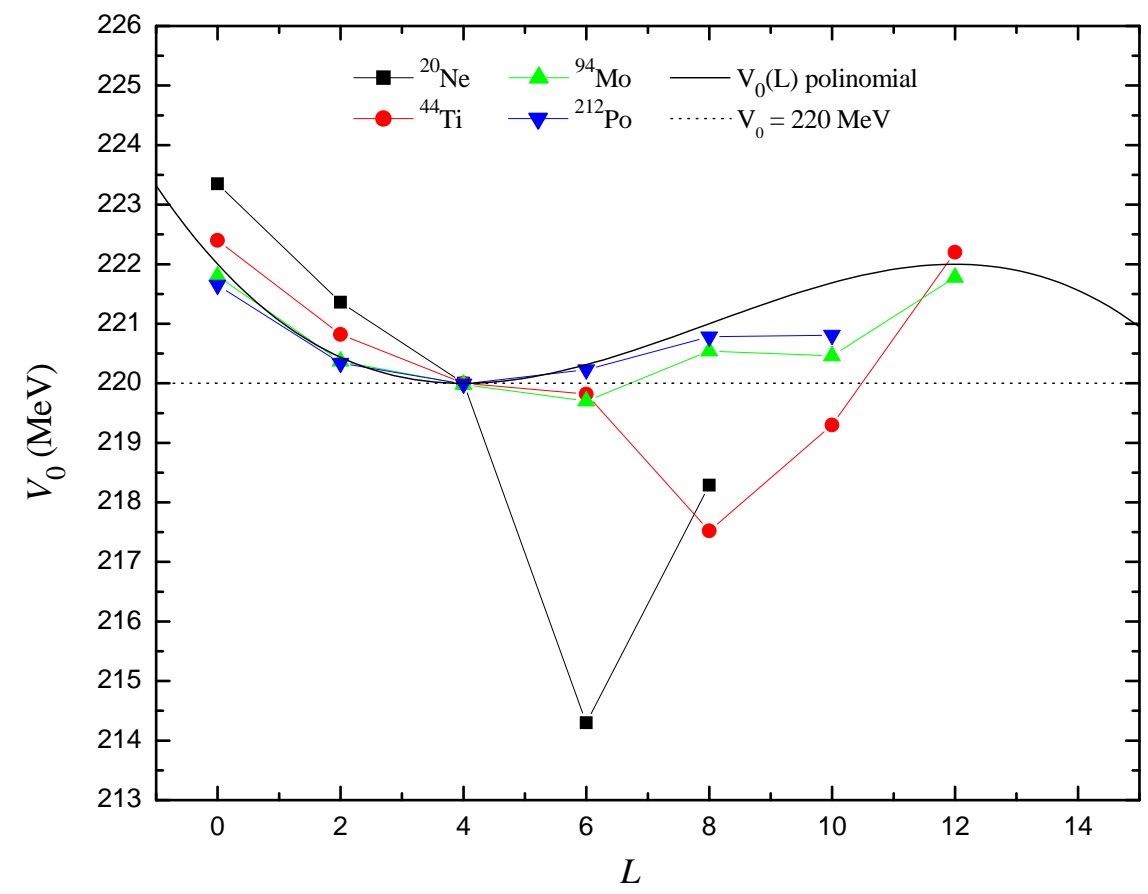

Figura 4.3: Profundidades $V_{0}$ ajustadas para a reprodução dos níveis experimentais das bandas de estado fundamental dos núcleos ${ }^{20} \mathrm{Ne},{ }^{44} \mathrm{Ti},{ }^{94} \mathrm{Mo} \mathrm{e}{ }^{212} \mathrm{Po}$ em função do número quântico $L$. A linha preta contínua mostra a função $V_{0}(L)$ na forma polinomial (ver eq. (4.5)) com seus pontos de mínimo e máximo local escolhidos adequadamente para uma descrição satisfatória dos gráficos $V_{0} \times L$. A linha pontilhada indica o valor constante $V_{0}=220 \mathrm{MeV}$ usado para a descrição geral dos espectros dos cinco núcleos.

$$
\left(L_{\min }, V_{0}^{\min }\right)=(4,220.0) \quad \text { e } \quad\left(L_{\max }, V_{0}^{\max }\right)=(12,222.0)
$$

A escolha de $\left(L_{\min }, V_{0}^{\min }\right)$ é justificada pelas seguintes características: 1) os quatro gráficos $V_{0} \times L$ apresentam pontos em $(4,220.0)$ (considerando uma precisão de décimos de $\mathrm{MeV})$; 2) o ponto de mínimo em (4,220.0) é favorável para a descrição do comportamento decrescente de $V_{0}$ em $L<4$ e do comportamento geral crescente de $V_{0}$ em $L>4$ para os pontos referentes ao ${ }^{94} \mathrm{Mo}$ e ${ }^{212} \mathrm{Po}$. Quanto à escolha de $\left(L_{\max }, V_{0}^{\max }\right)$, ela é justificada pelas características: 1) há pontos de máximo local em $L=12$ para os núcleos ${ }^{44} \mathrm{Ti}$ e ${ }^{94} \mathrm{Mo}$; 2) o valor $V_{0}^{\max }=222.0 \mathrm{MeV}$ é aproximadamente o valor médio das profundidades $V_{0}$ dos núcleos ${ }^{44} \mathrm{Ti}$ e ${ }^{94} \mathrm{Mo} \mathrm{em}$ $L=12$.

A Figura 4.3 mostra graficamente a função $V_{0}(L)$ obtida com os valores propostos para $\left(L_{\min }, V_{0}^{\min }\right)$ e $\left(L_{\max }, V_{0}^{\max }\right)$. No intervalo $0 \leq L \leq 4$, a função $V_{0}(L)$ descreve satisfatoriamente o comportamento decrescente de $V_{0}$, principalmente com relação aos núcleos ${ }^{44} \mathrm{Ti},{ }^{94} \mathrm{Mo} \mathrm{e}{ }^{212}$ Po. Para $L>4$, a descrição dos pontos relacionados ao ${ }^{212}$ Po também é satisfatória, enquanto a descrição dos pontos do 
${ }^{94}$ Mo é razoável. Com relação aos pontos do ${ }^{20} \mathrm{Ne}$ e ${ }^{44} \mathrm{Ti}$ em $L>4$, a função $V_{0}(L)$ produz uma descrição geral insatisfatória, com exceção dos pontos do ${ }^{44} \mathrm{Ti}$ em $L=6$ e $L=12$. Apesar haver diferenças locais muito grandes entre os gráficos $V_{0} \times L$, a função $V_{0}(L)$ ainda apresenta um desempenho geral satisfatório, pois a maioria dos pontos $\left(L, V_{0}\right)$ foram descritos mais eficientemente por $V_{0}(L)$ do que pelo valor constante de $220 \mathrm{MeV}$. Assim, $V_{0}(L)$ pode ser tratado como um termo de correção razoável para o potencial $\alpha+$ caroço das diferentes regiões de massa.

\subsection{Comportamento do parâmetro $R$ do potencial $\alpha+$ caroço}

\subsubsection{Análise do parâmetro $R$ na região do Molibdênio}

Utilizando os valores do parâmetro $R$ associados aos núcleos estudados neste trabalho, é possível verificar se este parâmetro possui relação com outras variáveis do sistema. Neste trabalho, é estudada a relação entre $R$ e o raio nuclear, além da influência do número quântico $G$ na variação de $R$.

A Figura 4.4 apresenta os valores de $R$ obtidos para os núcleos da região do Mo em dois gráficos, sendo que o gráfico 4.4(a) relaciona $R \operatorname{com} A_{T}^{1 / 3}\left(A_{T}\right.$ : número de massa do núcleo total) e o gráfico 4.4 (b) relaciona $R$ com a soma $A_{\alpha}^{1 / 3}+A_{\text {car }}^{1 / 3}\left(A_{\text {car }}\right.$ : número de massa do caroço). Com o uso do programa computacional ORIGIN $6.0^{1}$, foram realizados ajustes lineares do tipo

$$
y(x)=c_{1} x+c_{2} \quad \text { e } \quad y(x)=c_{3} x,
$$

onde $c_{1}, c_{2}$ e $c_{3}$ são os parâmetros de ajuste, com base nos pontos apresentados nos dois gráficos. Os valores obtidos para os três parâmetros foram:

$c_{1}=4.723 \mathrm{fm}, c_{2}=-15.867 \mathrm{fm}$ e $c_{3}=1.234 \mathrm{fm}$ para o gráfico $R \times A_{T}^{1 / 3}$,

$c_{1}=4.588 \mathrm{fm}, c_{2}=-22.237 \mathrm{fm}$ e $c_{3}=0.924 \mathrm{fm}$ para o gráfico $R \times\left(A_{\alpha}^{1 / 3}+A_{\text {car }}^{1 / 3}\right)$.

No gráfico 4.4(a), os desvios padrão associados aos ajustes do tipo $y(x)=c_{1} x+c_{2}$ e $y(x)=c_{3} x$ foram de $\approx 0.149 \mathrm{fm}$ e $0.220 \mathrm{fm}$, respectivamente, enquanto que no gráfico 4.4(b), os desvios padrão associados aos mesmos tipos de ajuste foram de $\approx 0.149 \mathrm{fm}$ e $0.231 \mathrm{fm}$, respectivamente. Os coeficientes de correlação associados

\footnotetext{
${ }^{1}$ O programa ORIGIN 6.0 é um produto da empresa Microcal Software, Inc. (Northampton, Estados Unidos).
} 


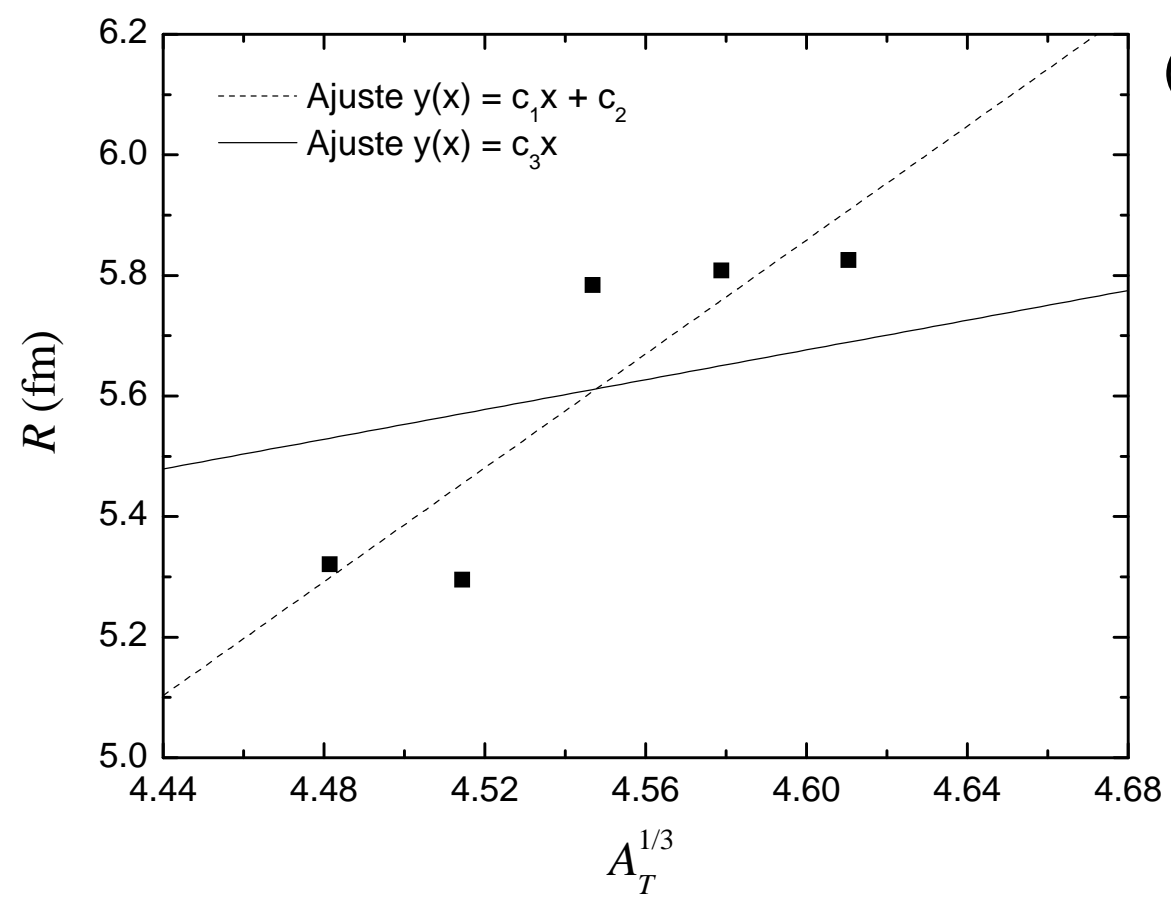

(a)

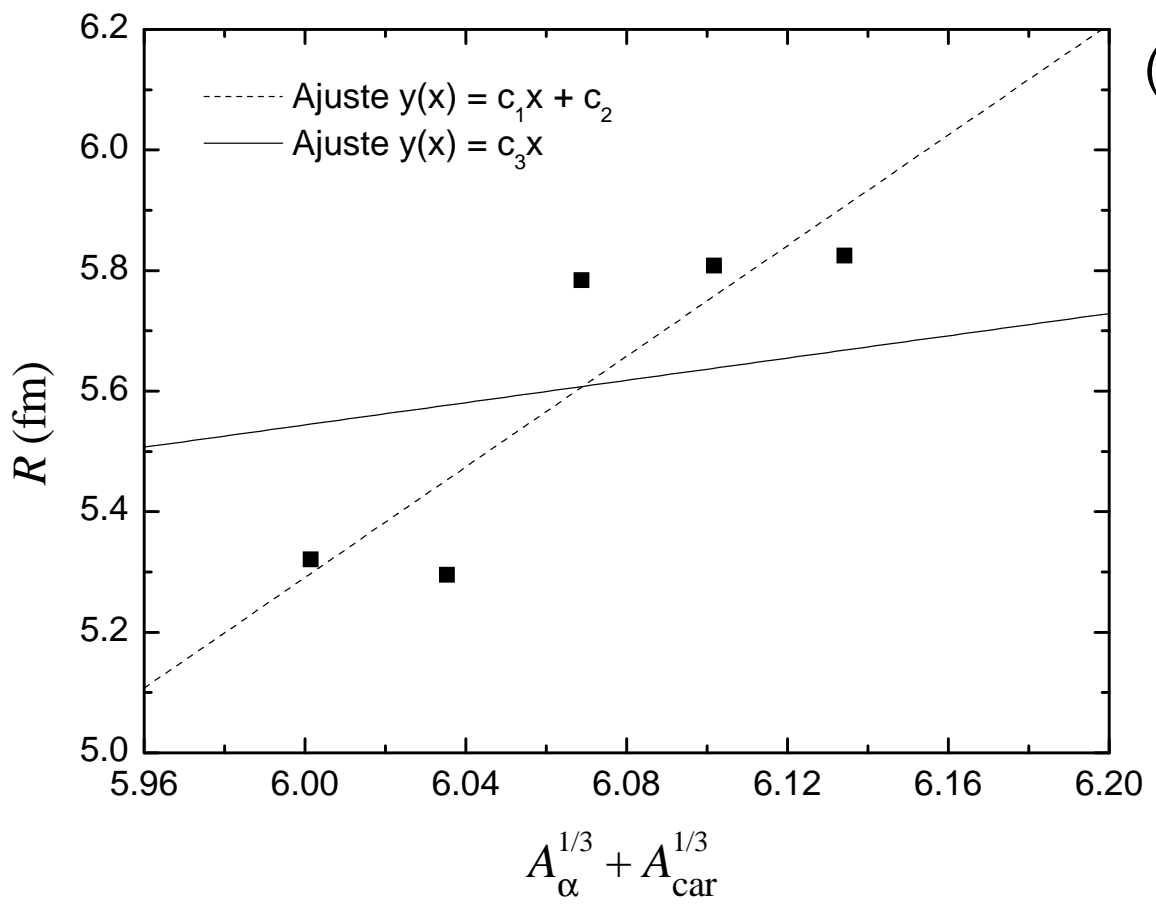

(b)

Figura 4.4: Valores do parâmetro $R$ para os núcleos ${ }^{90} \mathrm{Sr},{ }^{92} \mathrm{Zr},{ }^{94} \mathrm{Mo},{ }^{96} \mathrm{Ru}$ e ${ }^{98} \mathrm{Pd}$ apresentados em função de $A_{T}^{1 / 3}$ (a) e $A_{\alpha}^{1 / 3}+A_{\text {car }}^{1 / 3}(\mathbf{b})$. As retas tracejadas e contínuas representam os ajustes lineares do tipo $y(x)=c_{1} x+c_{2}$ e $y(x)=c_{3} x$, respectivamente, obtidos com referência nos pontos apresentados em cada gráfico. Os valores de $R$ são apresentados numericamente na Tabela 3.1. 
aos pontos dos gráficos $4.4(\mathrm{a})$ e $4.4(\mathrm{~b})$ são muito próximos, sendo $r \approx 0.882$ para ambos.

Apesar dos ajustes lineares apresentarem desvios padrão com valores relativos baixos e dos coeficientes de correlação indicarem uma tendência linear, não se pode afirmar previamente que as funções ajustadas sejam aplicáveis a núcleos mais afastados do intervalo $90 \leq A \leq 98$, pois as mesmas se referem a um pequeno conjunto de núcleos restritos à região de massa do Mo (na subseção seguinte, é feita a análise dos raios $R$ de outras regiões de massa). Além disto, as funções lineares ajustadas servem apenas como referências para uma estimativa inicial dos raios $R$, pois erros da ordem de $0.2 \mathrm{fm}$ no parâmetro $R$ alteram os níveis de energia do sistema $\alpha+$ caroço em vários $\mathrm{MeV}$.

Outro fato observado através da Figura 4.4 é o distanciamento de $\approx 0.5 \mathrm{fm}$ entre o grupo formado pelos pontos de ${ }^{90} \mathrm{Sr}$ e ${ }^{92} \mathrm{Zr}$ e o grupo formado pelos pontos de ${ }^{94} \mathrm{Mo},{ }^{96} \mathrm{Ru}$ e ${ }^{98} \mathrm{Pd}$. Tal fato está relacionado aos números quânticos $G$ associados às bandas de estado fundamental dos cinco núcleos $\left(G=14\right.$ para ${ }^{90} \mathrm{Sr}$ e ${ }^{92} \mathrm{Zr}$, e $G=16$ para ${ }^{94} \mathrm{Mo},{ }^{96} \mathrm{Ru}$ e $\left.{ }^{98} \mathrm{Pd}\right)$. Há uma diferença de energia de vários $\mathrm{MeV}$ entre dois estados teóricos $|G, L\rangle$ e $|G+2, L\rangle$ produzidos por um mesmo potencial $V(r)$ com parâmetros fixos; assim, há a necessidade de que os cinco núcleos tenham os seus respectivos parâmetros $R$ modificados, de modo que as energias dos estados teóricos $|G, L\rangle$ e $|G+2, L\rangle$ sejam ajustadas para a equiparação com os níveis experimentais $L^{\pi}$ correspondentes. O aumento de $R$ produz o "abaixamento" dos níveis de energia teóricos em relação ao limiar $\alpha+$ caroço, enquanto a redução de $R$ produz a "elevação" dos níveis de energia teóricos. O fato do aumento de $G$ implicar no aumento do parâmetro $R$ também afeta outras propriedades dos sistemas $\alpha+$ caroço, como é discutido nas seções seguintes deste capítulo.

\subsubsection{Análise do parâmetro $R$ em diferentes regiões de massa}

Os raios $R$ calculados para os núcleos ${ }^{20} \mathrm{Ne},{ }^{44} \mathrm{Ti}$ e ${ }^{212} \mathrm{Po}$ (ver Tabela 4.2 ) permitem outra comparação de maior abrangência em relação às diferentes regiões de massa. A Figura 4.5 apresenta os valores de $R$ obtidos para os núcleos ${ }^{20} \mathrm{Ne},{ }^{44} \mathrm{Ti}$, ${ }^{94} \mathrm{Mo} \mathrm{e}{ }^{212} \mathrm{Po}$ em dois gráficos, sendo que o gráfico 4.5 (a) relaciona $R \operatorname{com} A_{T}^{1 / 3}$ e o gráfico $4.5(\mathrm{~b})$ relaciona $R$ com a soma $A_{\alpha}^{1 / 3}+A_{\text {car }}^{1 / 3}$ Com o uso do programa ORIGIN 6.0, foram realizados os mesmos ajustes lineares indicados em (4.14) com base nos pontos referentes aos últimos quatro núcleos mencionados. Os valores obtidos para os três parâmetros de ajuste foram:

$c_{1}=1.140 \mathrm{fm}, c_{2}=0.382 \mathrm{fm} \mathrm{e} c_{3}=1.224 \mathrm{fm}$ para o gráfico $R \times A_{T}^{1 / 3}$,

$c_{1}=1.092 \mathrm{fm}, c_{2}=-1.041 \mathrm{fm}$ e $c_{3}=0.918 \mathrm{fm}$ para o gráfico $R \times\left(A_{\alpha}^{1 / 3}+A_{\text {car }}^{1 / 3}\right)$. 


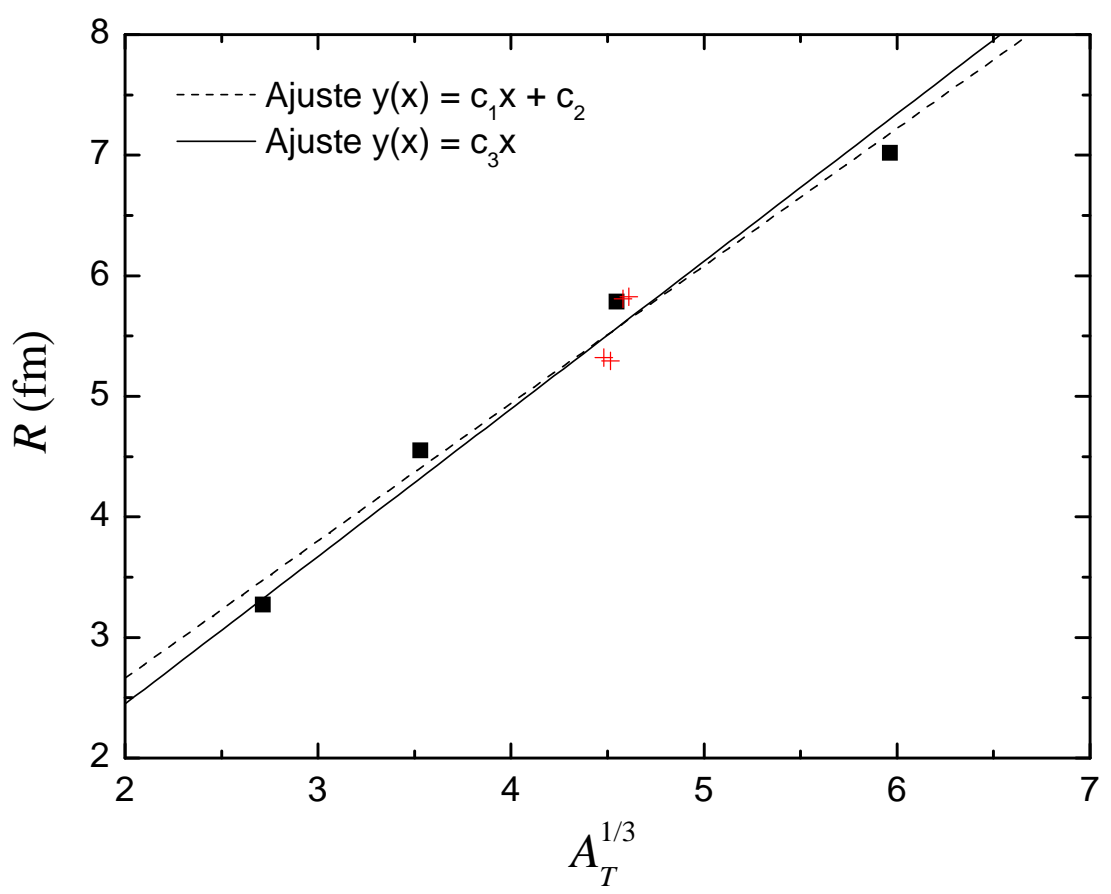

(a)

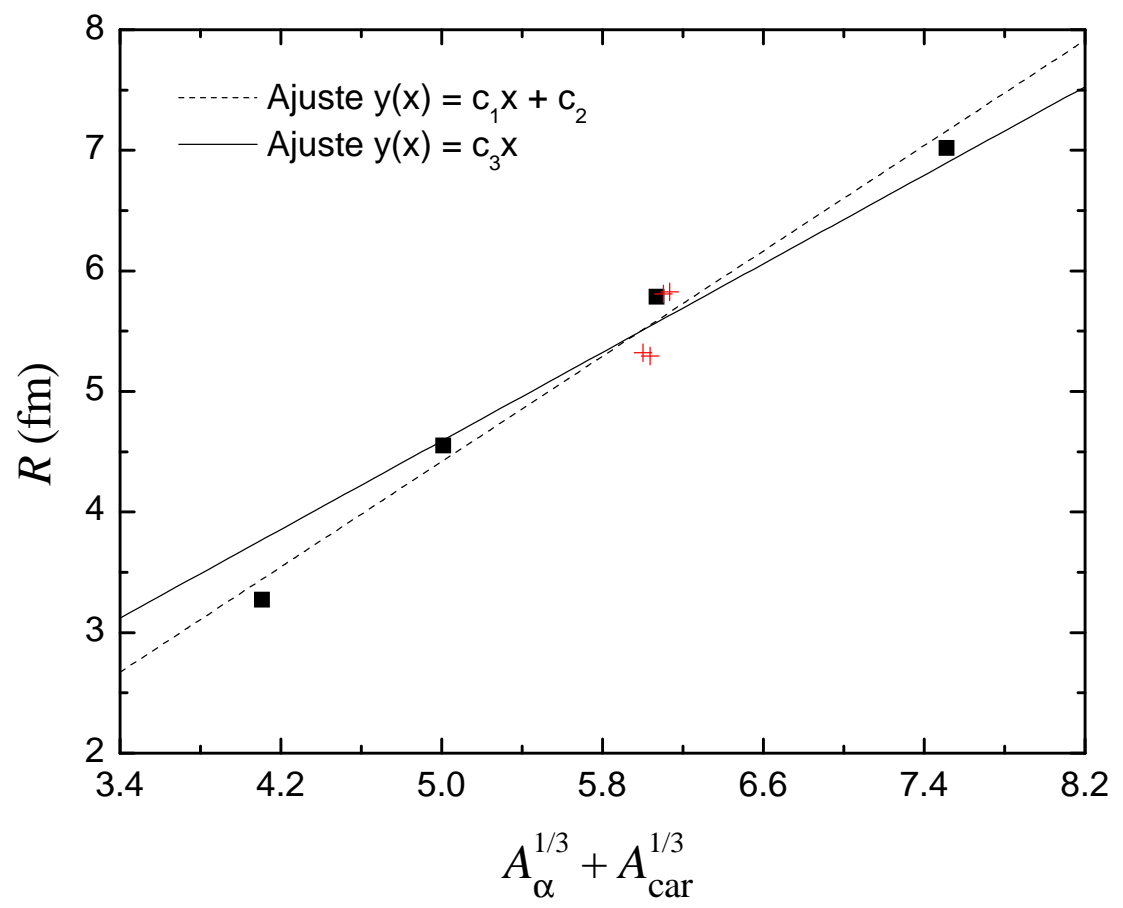

(b)

Figura 4.5: Valores do parâmetro $R$ para os núcleos ${ }^{20} \mathrm{Ne},{ }^{44} \mathrm{Ti},{ }^{94} \mathrm{Mo} \mathrm{e}{ }^{212} \mathrm{Po}$ (quadrados pretos) apresentados em função de $A_{T}^{1 / 3}$ (a) e $A_{\alpha}^{1 / 3}+A_{\text {car }}^{1 / 3}$ (b). As retas tracejadas e contínuas representam os ajustes lineares do tipo $y(x)=c_{1} x+c_{2}$ e $y(x)=c_{3} x$, respectivamente, obtidos com referência nos pontos dos núcleos citados inicialmente. Para efeito de comparação, são apresentados os pontos referentes aos raios $R$ dos núcleos ${ }^{90} \mathrm{Sr},{ }^{92} \mathrm{Zr},{ }^{96} \mathrm{Ru}$ e ${ }^{98} \mathrm{Pd}$ (cruzes vermelhas). Os valores de $R$ são apresentados numericamente nas Tabelas 3.1 e 4.2 . 
No gráfico 4.5(a), os desvios padrão associados aos ajustes do tipo $y(x)=c_{1} x+c_{2}$ e $y(x)=c_{3} x$ foram de $\approx 0.261 \mathrm{fm}$ e $0.246 \mathrm{fm}$, respectivamente, enquanto que no gráfico 4.5(b), os desvios padrão associados aos mesmos tipos de ajuste foram de $\approx 0.229 \mathrm{fm}$ e $0.322 \mathrm{fm}$, respectivamente. Os pontos referentes aos núcleos ${ }^{20} \mathrm{Ne}$, ${ }^{44} \mathrm{Ti},{ }^{94} \mathrm{Mo}$ e ${ }^{212} \mathrm{Po}$ resultam nos coeficientes de correlação 0.991 e 0.993 para os gráficos $4.5(\mathrm{a})$ e $4.5(\mathrm{~b})$, respectivamente, mostrando uma alta tendência linear em ambos os gráficos.

Os dois gráficos da Figura 4.5 mostram adicionalmente os raios $R$ referentes aos núcleos ${ }^{90} \mathrm{Sr},{ }^{92} \mathrm{Zr},{ }^{96} \mathrm{Ru}$ e ${ }^{98} \mathrm{Pd}$, os quais não foram considerados nos ajustes de reta descritos no parágrafo anterior. Verifica-se que os pontos referentes aos quatro núcleos da região do Mo encontram-se próximos das funções ajustadas, demonstrando que seguem a tendência linear dos pontos referentes aos núcleos ${ }^{20} \mathrm{Ne},{ }^{44} \mathrm{Ti},{ }^{94} \mathrm{Mo}$ e ${ }^{212}$ Po. Portanto, há compatibilidade entre o comportamento geral do parâmetro $R$ nas diferentes regiões de massa e o comportamento local de $R$ na região do Mo.

A compatibilidade dos comportamentos geral e local de $R$ também pode ser constatada pela comparação entre os ajustes lineares feitos especificamente na região do Mo e os ajustes descritos nesta subseção. Observando os parâmetros $c_{1}, c_{2}$ e $c_{3}$ apresentados nas Subseções 4.2 .1 e 4.2.2, constata-se que os ajustes lineares do tipo $y(x)=c_{1} x+c_{2}$ nos casos geral e local não são compatíveis; porém, nos ajustes lineares do tipo $y(x)=c_{3} x$, onde as funções ajustadas devem obrigatoriamente passar pela origem, há um alto grau de compatibilidade nos casos geral e local, pois os parâmetros $c_{3}$ calculados nos 2 casos são muito próximos (a razão entre os valores de $c_{3}$ nos casos local e geral são: 1.008 nos ajustes em $R \times A_{T}^{1 / 3}$ e 1.006 nos ajustes em $\left.R \times\left(A_{\alpha}^{1 / 3}+A_{\text {car }}^{1 / 3}\right)\right)$. Assim, pode-se afirmar que as relações de proporcionalidade

$$
R=1.224 A_{T}^{1 / 3} \mathrm{fm} \quad \text { e } \quad R=0.918\left(A_{\alpha}^{1 / 3}+A_{\text {car }}^{1 / 3}\right) \mathrm{fm},
$$

descrevem simultaneamente o comportamento do raio $R$ no caso geral (em diferentes regiões de massa) e local (na região do Mo), sendo que a primeira relação produz os menores desvios padrão. Contudo, a relação que melhor descreve os raios $R$ dos núcleos ${ }^{20} \mathrm{Ne},{ }^{44} \mathrm{Ti},{ }^{94} \mathrm{Mo}$ e ${ }^{212} \mathrm{Po}$ é

$$
R=1.092\left(A_{\alpha}^{1 / 3}+A_{\text {car }}^{1 / 3}\right)-1.041(\mathrm{fm}),
$$

de acordo com os desvios padrão calculados. Mais uma vez, deve-se lembrar que os baixos erros relativos cometidos em $R$ pelas funções ajustadas podem afetar drasticamente os espectros dos sistemas $\alpha+$ caroço, de modo que as funções citadas em (4.15) e (4.16) servem somente como fórmulas estimativas.

Como já foi discutido na Subseção 4.2.1, o número quântico $G$ exerce influência 
sobre o parâmetro $R$; porém, os gráficos da Figura 4.5 mostram que a variação local de $G$ resulta apenas em flutuações dos pontos $\left(R, A_{\alpha}^{1 / 3}\right)$ e $\left(R,\left(A_{\alpha}^{1 / 3}+A_{\text {car }}^{1 / 3}\right)\right)$ em torno das retas ajustadas, portanto, não afetando significativamente a relação linear (ou de proporcionalidade) entre $R$ e $A_{\alpha}^{1 / 3}$ e entre $R$ e $A_{\alpha}^{1 / 3}+A_{\text {car }}^{1 / 3}$.

\subsection{Separações intercluster rms}

As funções de onda radiais $u_{N, L}(r)$ associadas aos estados dos sistemas $\alpha+$ caroço podem ser usadas para o cálculo de diferentes propriedades. Lembrando que a coordenada $r$ representa a distância entre os centros de massa do cluster- $\alpha$ e do caroço, as funções $u_{N, L}(r)$ são usadas para o cálculo da quantidade

$$
\left\langle r^{2}\right\rangle_{N, L}=\int_{0}^{\infty} r^{2} u_{N, L}^{2}(r) d r
$$

onde $\left\langle r^{2}\right\rangle_{N, L}$ é o raio quadrático médio intercluster de um estado $|N, L\rangle$. Assim, a separação (ou raio) intercluster rms é dada por $\left\langle r^{2}\right\rangle^{1 / 2}$.

Usando as funções $u_{N, L}(r)$ calculadas para os cinco sistemas $\alpha+$ caroço da região do Mo, foram obtidas as separações intercluster rms apresentadas na Tabela 4.3. Um fato observado nas separações rms dos núcleos ${ }^{92} \mathrm{Zr},{ }^{94} \mathrm{Mo},{ }^{96} \mathrm{Ru}$ e ${ }^{98} \mathrm{Pd}$ é a existência de um valor máximo nos estados $2^{+}$, seguido de uma queda contínua de $\left\langle r^{2}\right\rangle^{1 / 2}$ até o estado de maior spin. ${ }^{2}$ Este comportamento, chamado na literatura como antistretching effect, já foi constatado em cálculos da estrutura de cluster- $\alpha$ em outras regiões de massa $[1,2,4,14]$ e no núcleo ${ }^{94} \mathrm{Mo}$ [4] com diferentes formas de potencial $\alpha+$ caroço. De acordo com observações de Buck, Dover e Vary [1], o efeito antistretching é esperado em sistemas onde o potencial $V(r)$ apresenta uma difusividade efetiva acentuada, tal como na forma do potencial $\alpha+$ caroço empregado neste trabalho.

A Tabela 4.3 mostra os valores calculados para a razão

$$
\frac{\left\langle r^{2}\right\rangle^{1 / 2}}{\left\langle R^{2}\right\rangle_{\alpha}^{1 / 2}+\left\langle R^{2}\right\rangle_{\text {car }}^{1 / 2}},
$$

onde $\left\langle R^{2}\right\rangle_{\alpha}^{1 / 2}$ e $\left\langle R^{2}\right\rangle_{\text {car }}^{1 / 2}$ são os raios rms experimentais [47] das distribuições de carga do cluster- $\alpha$ e do caroço, respectivamente. Observando os valores da razão descrita em (4.18) para os cinco núcleos, indica-se que há uma superposição entre o cluster- $\alpha$ e o caroço que já é significativa nos estados $0^{+}$e torna-se maior nos

\footnotetext{
${ }^{2}$ No núcleo ${ }^{90} \mathrm{Sr}$, não é possível confirmar o decrescimento de $\left\langle r^{2}\right\rangle^{1 / 2}$ devido à falta de níveis experimentais conhecidos acima de $4^{+}$. Contudo, o cálculo das separações rms para os níveis experimentais $E_{x}=2.9277 \mathrm{MeV}$ e $E_{x}=3.9543 \mathrm{MeV}$, onde sugere-se uma associação aos estados teóricos $6^{+}$e $8^{+}$, respectivamente, indica a presença do efeito antistretching no ${ }^{90} \mathrm{Sr}$.
} 
Tabela 4.3: Valores calculados para a separação intercluster $\operatorname{rms}\left(\left\langle r^{2}\right\rangle^{1 / 2}\right)$, a razão entre a separação intercluster rms e a soma dos raios rms experimentais do cluster- $\alpha$ e do caroço $\left(\left\langle r^{2}\right\rangle^{1 / 2} /\left(\left\langle R^{2}\right\rangle_{\alpha}^{1 / 2}+\left\langle R^{2}\right\rangle_{\text {car }}^{1 / 2}\right)\right)$, a largura- $\alpha$ reduzida $\left(\gamma_{\alpha}^{2}\right)$ e a largura- $\alpha$ reduzida adimensional $\left(\theta_{\alpha}^{2}\right)$, referentes às bandas de estado fundamental dos núcleos ${ }^{90} \mathrm{Sr},{ }^{92} \mathrm{Zr},{ }^{94} \mathrm{Mo},{ }^{96} \mathrm{Ru}$ e ${ }^{98} \mathrm{Pd}$. Os raios de canal usados para o cálculo de $\gamma_{\alpha}^{2}$ e $\theta_{\alpha}^{2}$ são obtidos por meio da eq. (4.24). Os raios rms experimentais do cluster- $\alpha$ e dos cinco núcleos-caroço são extraídos da Ref. [47].

\begin{tabular}{ccccc}
\hline \hline \multicolumn{5}{c}{ Núcleo: ${ }^{90} \mathrm{Sr}$} \\
$J^{\pi}$ & $\left\langle r^{2}\right\rangle^{1 / 2}(\mathrm{fm})$ & $\left\langle r^{2}\right\rangle^{1 / 2} /\left(\left\langle R^{2}\right\rangle_{\alpha}^{1 / 2}+\left\langle R^{2}\right\rangle_{\text {car }}^{1 / 2}\right)$ & $\gamma_{\alpha}^{2}(\mathrm{eV})$ & $\theta_{\alpha}^{2}\left(10^{-6}\right)$ \\
\hline $0^{+}$ & 4.650 & 0.794 & 90.60 & 408.7 \\
$2^{+}$ & 4.652 & 0.794 & 95.05 & 428.8 \\
$4^{+}$ & 4.623 & 0.789 & 73.58 & 331.9
\end{tabular}

Núcleo: ${ }^{92} \mathrm{Zr}$

\begin{tabular}{cccrr}
$J^{\pi}$ & $\left\langle r^{2}\right\rangle^{1 / 2}(\mathrm{fm})$ & $\left\langle r^{2}\right\rangle^{1 / 2} /\left(\left\langle R^{2}\right\rangle_{\alpha}^{1 / 2}+\left\langle R^{2}\right\rangle_{\text {car }}^{1 / 2}\right)$ & $\gamma_{\alpha}^{2}(\mathrm{eV})$ & $\theta_{\alpha}^{2}\left(10^{-6}\right)$ \\
\hline $0^{+}$ & 4.686 & 0.795 & 115.51 & 526.5 \\
$2^{+}$ & 4.695 & 0.796 & 126.89 & 578.3 \\
$4^{+}$ & 4.652 & 0.789 & 89.49 & 407.9 \\
$6^{+}$ & 5.610 & 0.782 & 61.56 & 280.6 \\
$\left(8^{+}\right)$ & 4.511 & 0.765 & 20.31 & 92.6 \\
$\left(10^{+}\right)$ & 4.426 & 0.751 & 5.57 & 25.4 \\
$\left(12^{+}\right)$ & 4.347 & 0.737 & 0.84 & 3.8 \\
$\left(14^{+}\right)$ & 4.310 & 0.731 & 0.07 & 0.3
\end{tabular}

Núcleo: ${ }^{94} \mathrm{Mo}$

\begin{tabular}{ccccc}
$J^{\pi}$ & $\left\langle r^{2}\right\rangle^{1 / 2}(\mathrm{fm})$ & $\left\langle r^{2}\right\rangle^{1 / 2} /\left(\left\langle R^{2}\right\rangle_{\alpha}^{1 / 2}+\left\langle R^{2}\right\rangle_{\text {car }}^{1 / 2}\right)$ & $\gamma_{\alpha}^{2}(\mathrm{eV})$ & $\theta_{\alpha}^{2}\left(10^{-6}\right)$ \\
\hline $0^{+}$ & 5.109 & 0.859 & 708.39 & 3261.8 \\
$2^{+}$ & 5.118 & 0.861 & 763.03 & 3513.4 \\
$4^{+}$ & 5.087 & 0.856 & 615.02 & 2831.8 \\
$6^{+}$ & 5.033 & 0.847 & 399.44 & 1839.2 \\
$8^{+}$ & 4.943 & 0.831 & 178.00 & 819.6 \\
$\left(10^{+}\right)$ & 4.859 & 0.817 & 66.15 & 304.6 \\
$\left(12^{+}\right)$ & 4.765 & 0.801 & 14.48 & 66.7 \\
\hline \hline
\end{tabular}


Tabela 4.3 (continuação)

\begin{tabular}{ccccc}
\hline \hline \multicolumn{5}{c}{ Núcleo: ${ }^{96} \mathrm{Ru}$} \\
$J^{\pi}$ & $\left\langle r^{2}\right\rangle^{1 / 2}(\mathrm{fm})$ & $\left\langle r^{2}\right\rangle^{1 / 2} /\left(\left\langle R^{2}\right\rangle_{\alpha}^{1 / 2}+\left\langle R^{2}\right\rangle_{\text {car }}^{1 / 2}\right)$ & $\gamma_{\alpha}^{2}(\mathrm{eV})$ & $\theta_{\alpha}^{2}\left(10^{-6}\right)$ \\
\hline $0^{+}$ & 5.103 & 0.852 & 595.69 & 2770.8 \\
$2^{+}$ & 5.112 & 0.853 & 636.52 & 2960.7 \\
$4^{+}$ & 5.081 & 0.848 & 509.85 & 2371.5 \\
$6^{+}$ & 5.020 & 0.838 & 310.25 & 1443.1 \\
$8^{+}$ & 4.943 & 0.825 & 149.95 & 697.5 \\
$10^{+}$ & 4.861 & 0.811 & 54.87 & 255.2 \\
$12^{+}$ & 4.776 & 0.797 & 13.11 & 61.0 \\
$14^{+}$ & 4.725 & 0.789 & 2.43 & 11.3 \\
$16^{+}$ & 4.702 & 0.785 & 0.20 & 0.9 \\
& & & & \\
& & Núcleo: ${ }^{98} \mathrm{Pd}$ & & \\
$J^{\pi}$ & $\left\langle r^{2}\right\rangle^{1 / 2}(\mathrm{fm})$ & $\left\langle r^{2}\right\rangle^{1 / 2} /\left(\left\langle R^{2}\right\rangle_{\alpha}^{1 / 2}+\left\langle R^{2}\right\rangle_{\text {car }}^{1 / 2}\right)$ & $\gamma_{\alpha}^{2}(\mathrm{eV})$ & $\theta_{\alpha}^{2}\left(10^{-6}\right)$ \\
\hline $0^{+}$ & 5.101 & 0.842 & 497.31 & 2341.9 \\
$2^{+}$ & 5.114 & 0.844 & 541.49 & 2549.9 \\
$4^{+}$ & 5.082 & 0.839 & 430.80 & 2028.6 \\
$\left(6^{+}\right)$ & 5.019 & 0.829 & 256.86 & 1209.6 \\
$\left(8^{+}\right)$ & 4.941 & 0.816 & 119.26 & 561.6 \\
$\left(10^{+}\right)$ & 4.859 & 0.802 & 43.28 & 203.8 \\
$\left(12^{+}\right)$ & 4.782 & 0.790 & 10.90 & 51.3 \\
$\left(14^{+}\right)$ & 4.732 & 0.781 & 2.01 & 9.5 \\
$\left(16^{+}\right)$ & 4.715 & 0.778 & & 0.17 \\
\hline \hline
\end{tabular}

últimos níveis das bandas de estado fundamental. Isto sugere que a banda do estado fundamental apresenta uma estrutura de cluster- $\alpha$ mais compacta nos níveis de maior spin, o que está de acordo com as previsões de S. Ohkubo [4], o qual utiliza um potencial nuclear do tipo double folding para a descrição da interação $\alpha+$ caroço no ${ }^{94} \mathrm{Mo}$.

Os valores de $\left\langle r^{2}\right\rangle^{1 / 2} /\left(\left\langle R^{2}\right\rangle_{\alpha}^{1 / 2}+\left\langle R^{2}\right\rangle_{\text {car }}^{1 / 2}\right)$ também indicam que há uma sensível diferença no grau de compactação das estruturas $\alpha+$ caroço dos cinco núcleos estudados, onde os núcleos ${ }^{90} \mathrm{Sr}$ e ${ }^{92} \mathrm{Zr}$ formam um grupo com um grau de compactação mais alto da estrutura $\alpha+\operatorname{caroço~}\left(\operatorname{com}\left\langle r^{2}\right\rangle^{1 / 2} /\left(\left\langle R^{2}\right\rangle_{\alpha}^{1 / 2}+\left\langle R^{2}\right\rangle_{\text {car }}^{1 / 2}\right) \approx 0.79\right.$ em seus estados fundamentais) e os núcleos ${ }^{94} \mathrm{Mo},{ }^{96} \mathrm{Ru}$ e ${ }^{98} \mathrm{Pd}$ formam outro grupo com um grau de compactação mais baixo (com $\left\langle r^{2}\right\rangle^{1 / 2} /\left(\left\langle R^{2}\right\rangle_{\alpha}^{1 / 2}+\left\langle R^{2}\right\rangle_{\text {car }}^{1 / 2}\right) \approx 0.85$ em seus estados fundamentais). Este fato está relacionado aos números quânticos $G$ cor- 
respondentes às bandas de estado fundamental. Como foi discutido na Subseção 4.2.1, os núcleos associados a $G=16$ possuem parâmetros $R$ aproximadamente $0.5 \mathrm{fm}$ maiores do que os núcleos associados a $G=14$, o que afeta diretamente a extensão das funções radiais $u_{N, L}(r)$ e, consequentemente, o comportamento da quantidade $\left\langle r^{2}\right\rangle^{1 / 2} /\left(\left\langle R^{2}\right\rangle_{\alpha}^{1 / 2}+\left\langle R^{2}\right\rangle_{\text {car }}^{1 / 2}\right)$. Analisando individualmente os cinco núcleos, nota-se que o ${ }^{94}$ Mo é aquele com o menor grau de compactação da estrutura $\alpha+$ caroço, corroborando as afirmações da literatura de que o ${ }^{94}$ Mo é o núcleo preferencial para a investigação da estrutura de cluster- $\alpha$ na sua região de massa.

Usando as separações rms $\left\langle r^{2}\right\rangle_{0^{+}}^{1 / 2}$ referentes aos estados fundamentais dos núcleos estudados e os raios rms experimentais $\left\langle R^{2}\right\rangle_{\alpha}^{1 / 2}$ e $\left\langle R^{2}\right\rangle_{\text {car }}^{1 / 2}$, pode-se estimar os raios rms $\left\langle R^{2}\right\rangle_{T}^{1 / 2}$ das distribuições de carga dos núcleos totais através da equação [8]:

$$
\begin{aligned}
\left\langle R^{2}\right\rangle_{T}=\frac{Z_{\alpha}}{Z_{\alpha}+Z_{\mathrm{car}}} & \left\langle R^{2}\right\rangle_{\alpha}+ \\
& \frac{Z_{\mathrm{car}}}{Z_{\alpha}+Z_{\mathrm{car}}}\left\langle R^{2}\right\rangle_{\mathrm{car}}+\frac{Z_{\alpha} A_{\mathrm{car}}^{2}+Z_{\mathrm{car}} A_{\alpha}^{2}}{\left(Z_{\alpha}+Z_{\mathrm{car}}\right)\left(A_{\alpha}+A_{\mathrm{car}}\right)^{2}}\left\langle r^{2}\right\rangle_{0^{+}} .
\end{aligned}
$$

O cálculo de $\left\langle R^{2}\right\rangle_{T}^{1 / 2}$ permite verificar se o potencial $\alpha+$ caroço empregado neste trabalho produz separações rms $\left\langle r^{2}\right\rangle_{0^{+}}^{1 / 2}$ compatíveis com os raios rms experimentais dos núcleos totais. Os raios $\left\langle R^{2}\right\rangle_{T}^{1 / 2}$ calculados para os núcleos ${ }^{90} \mathrm{Sr},{ }^{92} \mathrm{Zr}$, ${ }^{94} \mathrm{Mo}$ e ${ }^{96} \mathrm{Ru}$ são apresentados na Tabela 4.4, juntamente com as razões entre os valores calculados para $\left\langle R^{2}\right\rangle_{T}^{1 / 2}$ e os respectivos raios rms experimentais [47] de distribuição de carga dos núcleos totais. O raio $\left\langle R^{2}\right\rangle_{T}^{1 / 2}$ não foi calculado para o núcleo ${ }^{98} \mathrm{Pd}$, pois a literatura não apresenta raios rms experimentais para os núcleos ${ }^{94} \mathrm{Ru}$ e ${ }^{98} \mathrm{Pd}$. A Tabela 4.4 mostra que as razões $\left\langle R^{2}\right\rangle_{T}^{1 / 2} /\left\langle R^{2}\right\rangle_{T \text { exp }}^{1 / 2}$ possuem valores muito próximos de 1 , demonstrando um alto grau de compatibilidade das separações rms $\left\langle r^{2}\right\rangle_{0^{+}}^{1 / 2}$ com os raios rms experimentais dos núcleos totais.

\subsection{Larguras- $\alpha$ reduzidas}

\subsubsection{Definições referentes à largura reduzida}

Outra propriedade calculada por meio das funções de onda radiais $u_{N, L}(r)$ é a largura- $\alpha$ reduzida $[48,49]$

$$
\gamma_{\alpha ; N, L}^{2}=\left(\frac{\hbar^{2}}{2 \mu a_{c}}\right) u_{N, L}^{2}\left(a_{c}\right)\left[\int_{0}^{a_{c}}\left|u_{N, L}(r)\right|^{2} d r\right]^{-1},
$$

onde $\mu$ é a massa reduzida do sistema $\alpha+$ caroço e $a_{c}$ é o raio de canal. A largura- $\alpha$ reduzida adimensional é definida como a razão entre $\gamma_{\alpha ; N, L}^{2}$ e o limite de Wigner $[50,2]$, isto é: 
Tabela 4.4: Raios rms $\left\langle R^{2}\right\rangle_{T}^{1 / 2}$ calculados para os núcleos totais ${ }^{90} \mathrm{Sr},{ }^{92} \mathrm{Zr},{ }^{94} \mathrm{Mo}$ e ${ }^{96} \mathrm{Ru}$ através da eq. (4.19). Para cada núcleo total, é mostrada a razão entre o valor calculado $\left\langle R^{2}\right\rangle_{T}^{1 / 2}$ e o raio rms experimental [47] da distribuição de carga do mesmo núcleo $\left(\left\langle R^{2}\right\rangle_{T \exp }^{1 / 2}\right)$.

\begin{tabular}{ccc}
\hline \hline Núcleo total & $\left\langle R^{2}\right\rangle_{T}^{1 / 2}(\mathrm{fm})$ & $\left\langle R^{2}\right\rangle_{T}^{1 / 2} /\left\langle R^{2}\right\rangle_{T \exp }^{1 / 2}$ \\
\hline${ }^{90} \mathrm{Sr}$ & 4.220 & 0.990 \\
${ }^{92} \mathrm{Zr}$ & 4.254 & 0.988 \\
${ }^{94} \mathrm{Mo}$ & 4.322 & 0.993 \\
${ }^{96} \mathrm{Ru}$ & 4.363 & 0.993 \\
\hline \hline
\end{tabular}

$$
\theta_{\alpha ; N, L}^{2}=\frac{2 \mu a_{c}^{2}}{3 \hbar^{2}} \gamma_{\alpha ; N, L}^{2}
$$

A largura- $\alpha$ reduzida pode ser interpretada como uma medida da intensidade de aglomeração- $\alpha$ no estado $|N, L\rangle$. No caso da largura- $\alpha$ reduzida adimensional, um valor $\theta_{\alpha ; N, L}^{2} \approx 1$ indica qualitativamente uma forte característica de aglomeração- $\alpha$ no estado $|N, L\rangle$. O raio de canal define a região $r>a_{c}$ onde a interação nuclear torna-se desprezível e a interação coulombiana é predominante.

Na perspectiva da teoria da matriz- $R$ [48], a largura- $\alpha$ reduzida de um estado $|N, L\rangle$ está relacionada à largura- $\alpha$ total $\Gamma_{\alpha ; N, L}$ por $^{3}$

$$
\Gamma_{\alpha ; N, L}=2 P\left(a_{c}\right) \gamma_{\alpha ; N, L}^{2}
$$

onde $P\left(a_{c}\right)$ é a penetrabilidade, dada por

$$
P\left(a_{c}\right)=\frac{k a_{c}}{F_{L}^{2}\left(k a_{c}\right)+G_{L}^{2}\left(k a_{c}\right)},
$$

sendo que $F_{L}$ e $G_{L}$ são as funções de Coulomb esféricas nas formas regular e irregular, respectivamente, e $k$ é o número de onda associado ao movimento relativo $\alpha+$ caroço.

A definição em (4.22) é aplicada exclusivamente para estados ressonantes, pois a largura- $\alpha$ total $\Gamma_{\alpha}$ e a penetrabilidade $P\left(a_{c}\right)$ são propriedades associadas a estados acima do limiar $\alpha+$ caroço. Contudo, a largura- $\alpha$ reduzida pode ser calculada para estados ligados, mesmo estando fora do contexto apresentado na eq. (4.22). Observando a eq. (4.20), nota-se que a determinação de $\gamma_{\alpha ; N, L}^{2}$ depende da função

\footnotetext{
${ }^{3}$ De acordo com a Ref. [48], o uso da eq. (4.22) é conveniente apenas para larguras $\Gamma_{\alpha}$ estreitas pois, em outros casos, a eq. (4.22) demonstra ser instável diante da variação do raio de canal $a_{c}$. Porém, várias referências sobre este tema (exemplos nas Refs. [1, 49, 50]) citam a eq. (4.22) como uma fórmula básica para o estabelecimento da relação entre $\Gamma_{\alpha}$ e $\gamma_{\alpha}^{2}$.
} 
$u_{N, L}(r)$ no intervalo $0 \leq r \leq a_{c}$. Dentro do referido intervalo, as funções de onda radiais associadas a estados ligados e ressonantes apresentam um comportamento similar, sendo oscilatório na região do poço de potencial $(E>V(r))$ e exponencial decrescente na região da barreira de potencial efetivo $(E<V(r))$. Portanto, as características relevantes de $u_{N, L}(r)$ para a determinação de $\gamma_{\alpha ; N, L}^{2}$ são as mesmas em ambos os casos. Neste trabalho, a eq. (4.22) é aplicada igualmente para estados ligados e ressonantes, considerando que $\gamma_{\alpha ; N, L}^{2}$ fornece o mesmo tipo de informação sobre a estrutura de cluster- $\alpha$ para estados acima ou abaixo do limiar $\alpha+$ caroço. $^{4}$

\subsubsection{Critério para a escolha do raio de canal}

Os trabalhos da literatura sobre estruturas de cluster não apresentam um critério comum para a escolha do raio de canal num certo sistema cluster + caroço. Um aspecto presente nos trabalhos mencionados é a escolha de $a_{c}$ em torno do raio correspondente ao topo da barreira de potencial; contudo, não há um procedimento que defina quanto o valor de $a_{c}$ deve estar próximo do raio do topo da barreira.

Para que os valores de $\gamma_{\alpha}^{2}$ e $\theta_{\alpha}^{2}$ sejam comparados entre diferentes núcleos, é necessário que $a_{c}$ seja definido através de um critério único para todos os sistemas $\alpha+$ caroço. Desta forma, os raios de canal adotados na literatura para a análise dos sistemas $\alpha+{ }^{16} \mathrm{O}\left(a_{c}=6 \mathrm{fm}[13,48]\right), \alpha+{ }^{40} \mathrm{Ca}\left(a_{c}=7.5 \mathrm{fm}[2]\right)$ e $\alpha+{ }^{208} \mathrm{~Pb}\left(a_{c}=\right.$ $10.5 \mathrm{fm}[4])$ foram utilizados como referências para a obtenção de uma função que defina o raio $a_{c}$ em qualquer sistema $\alpha+$ caroço. Os três raios de canal mencionados foram comparados graficamente, sendo demonstrado que os valores de $a_{c}$ tendem a se comportar linearmente em função dos seus respectivos valores de $A_{T}^{1 / 3}$ ou $A_{\alpha}^{1 / 3}+A_{\text {car }}^{1 / 3}$ (os coeficientes de correlação obtidos foram: 0.996 para $a_{c} \times A_{T}^{1 / 3} \mathrm{e}$ 0.997 para $\left.a_{c} \times\left(A_{\alpha}^{1 / 3}+A_{\text {car }}^{1 / 3}\right)\right)$. O ajuste linear ${ }^{5}$ que descreveu os três valores de $a_{c}$ com o menor desvio padrão foi:

$$
a_{c}=1.295\left(A_{\alpha}^{1 / 3}+A_{\text {car }}^{1 / 3}\right)+0.824 .
$$

A Figura 4.6 mostra o gráfico $a_{c} \times\left(A_{\alpha}^{1 / 3}+A_{\text {car }}^{1 / 3}\right)$ com os três raios de canal adotados na literatura, juntamente com a função linear correspondente à eq. (4.24). A mesma equação pode ser usada para estimar o raio $a_{c}$ em outros sistemas $\alpha+$ caroço, sendo coerente com os raios de canal já aplicados aos núcleos ${ }^{20} \mathrm{Ne},{ }^{44} \mathrm{Ti}$ e ${ }^{212} \mathrm{Po}$.

\footnotetext{
${ }^{4}$ Citando exemplos na literarura, as Refs. [9, 51, 52] mostram valores de larguras- $\alpha$ reduzidas (ou larguras- $\alpha$ reduzidas adimensionais) calculadas de forma igual para estados ligados e ressonantes de diferentes sistemas $\alpha+$ caroço, em diferentes contextos. Deve-se observar a existência de trabalhos da literatura baseados na teoria da matriz- $R$ que se restringem ao cálculo da largura reduzida para estados ressonantes.

${ }^{5} \mathrm{O}$ ajuste linear e os coeficientes de correlação mencionados na Subseção 4.4.2 foram obtidos por meio do programa ORIGIN 6.0.
} 


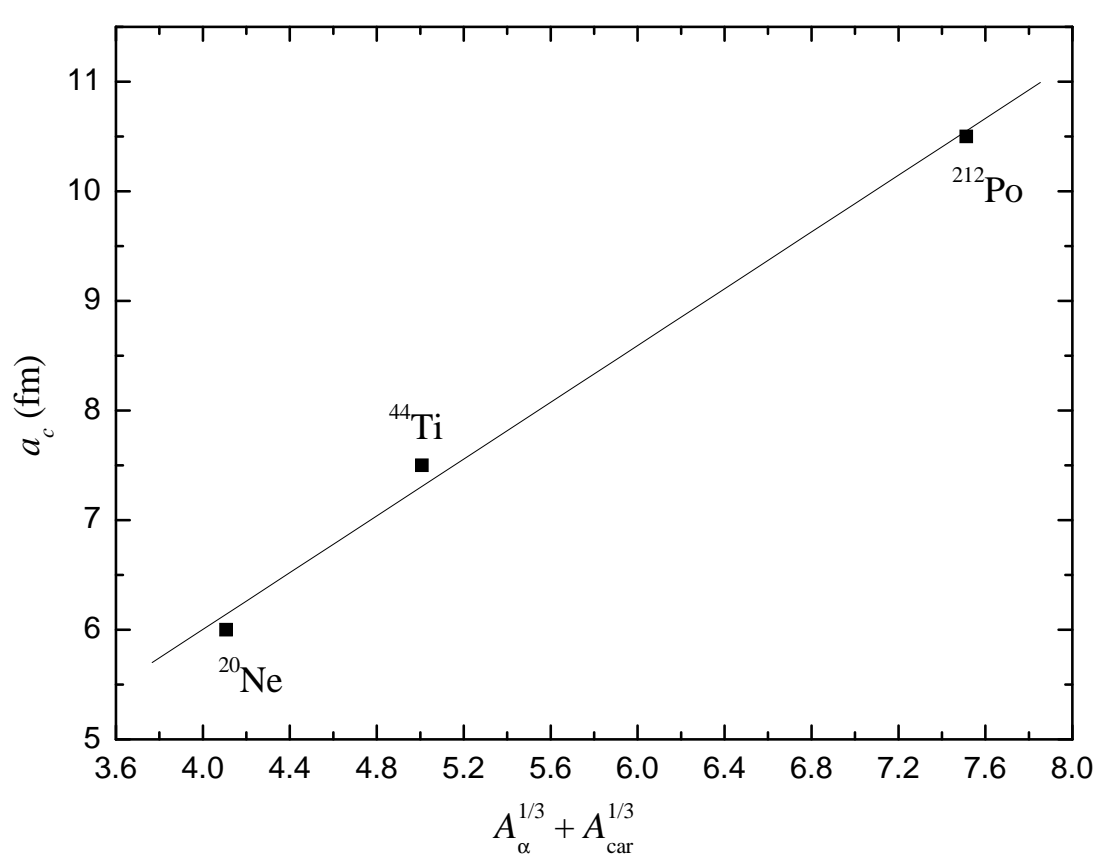

Figura 4.6: Raios de canal $a_{c}$ usados na literatura para os núcleos ${ }^{20} \mathrm{Ne}[48,13],{ }^{44} \mathrm{Ti}[2]$ e ${ }^{212} \mathrm{Po}$ [4] apresentados em função de $A_{\alpha}^{1 / 3}+A_{\text {car }}^{1 / 3}$. A reta contínua mostra o ajuste linear (ver eq. (4.24)) obtido a partir dos três pontos do gráfico.

\subsubsection{Resultados para as larguras- $\alpha$ reduzidas na região do Molibdênio}

A eq. (4.24) foi usada para a determinação dos raios $a_{c}$ associados aos núcleos totais ${ }^{90} \mathrm{Sr},{ }^{92} \mathrm{Zr},{ }^{94} \mathrm{Mo},{ }^{96} \mathrm{Ru}$ e ${ }^{98} \mathrm{Pd}$, sendo então aplicados na eq. (4.20) para o cálculo das larguras- $\alpha$ reduzidas nas bandas de estado fundamental. Os valores obtidos são mostrados na Tabela 4.3, juntamente com as larguras- $\alpha$ reduzidas adimensionais $\theta_{\alpha}^{2}$. A observação dos valores de $\gamma_{\alpha}^{2}$ revela que os estados de mais baixos spins possuem larguras- $\alpha$ que diferem daquelas obtidas nos estados de mais altos spins em algumas ordens de grandeza, indicando que os estados de menores spins dos cinco núcleos estão mais fortemente associados à estrutura de cluster- $\alpha$ do que aqueles de maiores spins. Porém, mesmo os valores mais altos obtidos para $\gamma_{\alpha}^{2}$ representam frações muito pequenas do limite de Wigner, como se demonstra pelas larguras- $\alpha$ reduzidas adimensionais $\theta_{\alpha}^{2}$. Portanto, uma análise geral dos valores de $\theta_{\alpha}^{2}$ indica que as bandas de estado fundamental dos cinco núcleos da região do Mo possuem uma fraca característica de aglomeração- $\alpha$. Apesar deste fato, deve-se levar em conta que as bandas de estado fundamental dos núcleos de outras regiões de massa também apresentam pequenos valores de $\theta_{\alpha}^{2}$, como é discutido em maiores detalhes na Subseção 4.4.4.

O comportamento de $\gamma_{\alpha}^{2}$ nos cinco núcleos da região do Mo está diretamente relacionado ao comportamento das separações intercluster rms nos mesmos núcleos. 
Conforme as discussões da Seção 4.3, as separações rms dos cinco núcleos apresentam valores máximos nos estados $2^{+}$, seguidos de uma queda contínua do valor de $\left\langle r^{2}\right\rangle^{1 / 2}$ para $J$ crescente. As larguras- $\alpha$ reduzidas também apresentam um comportamento decrescente a partir dos estados $2^{+}$, mostrando que os valores de $\left\langle r^{2}\right\rangle^{1 / 2}$ e $\gamma_{\alpha}^{2}$ fornecem a mesma informação a respeito da diminuição da aglomeração- $\alpha$ para $J$ crescente. ${ }^{6}$ Os números quânticos $G$ associados às bandas de estado fundamental afetam o comportamento de $\theta_{\alpha}^{2}$ de forma similar ao efeito observado nas razões $\left\langle r^{2}\right\rangle^{1 / 2} /\left(\left\langle R^{2}\right\rangle_{\alpha}^{1 / 2}+\left\langle R^{2}\right\rangle_{\text {car }}^{1 / 2}\right)$, pois verifica-se que os núcleos associados a $G=14\left({ }^{90} \mathrm{Sr}\right.$ e $\left.{ }^{92} \mathrm{Zr}\right)$ formam um grupo com intensidade de aglomeração- $\alpha$ mais baixa (onde $\theta_{\alpha}^{2}$ varia entre $\approx 4 \times 10^{-4} \mathrm{e} \approx 5 \times 10^{-4}$ em seus estados fundamentais) e os núcleos associados a $G=16\left({ }^{94} \mathrm{Mo},{ }^{96} \mathrm{Ru}\right.$ e $\left.{ }^{98} \mathrm{Pd}\right)$ formam outro grupo com intensidade de aglomeração- $\alpha$ mais alta (onde $\theta_{\alpha}^{2}$ varia entre $\approx 2 \times 10^{-3} \mathrm{e}$ $\approx 3 \times 10^{-3}$ em seus estados fundamentais). Portanto, a relação entre o parâmetro $R$ do potencial $\alpha+$ caroço e o número quântico $G$ (ver Subseção 4.2.1) é relevante no comportamento de $\gamma_{\alpha}^{2}$ e $\theta_{\alpha}^{2}$, assim como na razão $\left\langle r^{2}\right\rangle^{1 / 2} /\left(\left\langle R^{2}\right\rangle_{\alpha}^{1 / 2}+\left\langle R^{2}\right\rangle_{\text {car }}^{1 / 2}\right)$.

Comparando os estados $J^{+}$dos cinco núcleos da região do Mo, nota-se que o ${ }^{94} \mathrm{Mo}$ é o núcleo com os maiores valores de $\theta_{\alpha}^{2}$. Este fato, adicionado às constatações a respeito da razão $\left\langle r^{2}\right\rangle^{1 / 2} /\left(\left\langle R^{2}\right\rangle_{\alpha}^{1 / 2}+\left\langle R^{2}\right\rangle_{\text {car }}^{1 / 2}\right)$, corroboram as afirmações da literatura de que o ${ }^{94}$ Mo é o núcleo preferencial para a investigação da estrutura de cluster- $\alpha$ na sua região de massa.

\subsubsection{Comportamento das larguras- $\alpha$ reduzidas em outras regiões de massa}

É interessante comparar as larguras- $\alpha$ reduzidas dos núcleos da região do Mo com a mesma grandeza calculada para núcleos de outras regiões de massa. Com isto, pode-se fazer uma avaliação mais abrangente sobre as ordens de grandeza de $\gamma_{\alpha}^{2}$ e $\theta_{\alpha}^{2}$

As larguras- $\alpha$ reduzidas referentes às bandas de estado fundamental dos núcleos ${ }^{20} \mathrm{Ne},{ }^{44} \mathrm{Ti}$ e ${ }^{212} \mathrm{Po}$ foram calculadas. Os valores obtidos encontram-se na Tabela 4.5. Para o cálculo de $\gamma_{\alpha}^{2}$ e $\theta_{\alpha}^{2}$ neste caso, foi necessária a determinação das funções radiais $u_{N, L}(r)$ associadas aos níveis das bandas de estado fundamental dos três núcleos. As funções $u_{N, L}(r)$ foram calculadas com a mesma forma de potencial $\alpha+$ caroço aplicada aos núcleos da região do Mo, usando os raios $R$ e números quânticos $G$ descritos na Tabela 4.2 e as profundidades $V_{0}$ apresentadas graficamente na

\footnotetext{
${ }^{6}$ No núcleo ${ }^{90} \mathrm{Sr}$, não é possível confirmar o decrescimento de $\gamma_{\alpha}^{2}$ e $\theta_{\alpha}^{2}$ devido à falta de níveis experimentais conhecidos acima de $4^{+}$. Contudo, o cálculo das larguras- $\alpha$ reduzidas para os níveis experimentais $E_{x}=2.9277 \mathrm{MeV}$ e $E_{x}=3.9543 \mathrm{MeV}$, onde sugere-se uma associação aos estados teóricos $6^{+}$e $8^{+}$, respectivamente, indica o comportamento decrescente de $\gamma_{\alpha}^{2}$ e $\theta_{\alpha}^{2}$ para o ${ }^{90} \mathrm{Sr}$.
} 
Tabela 4.5: Valores calculados para a largura- $\alpha$ reduzida $\left(\gamma_{\alpha}^{2}\right)$ e a largura- $\alpha$ reduzida adimensional $\left(\theta_{\alpha}^{2}\right)$ referentes às bandas de estado fundamental dos núcleos ${ }^{20} \mathrm{Ne},{ }^{44} \mathrm{Ti} \mathrm{e}{ }^{212} \mathrm{Po}$. Adicionalmente, são mostradas as energias de excitação $E_{x}$ dos níveis experimentais. Os raios de canal usados para o cálculo de $\gamma_{\alpha}^{2}$ e $\theta_{\alpha}^{2}$ são obtidos por meio da eq. (4.24).

\begin{tabular}{cccc}
\hline \hline \multicolumn{4}{c}{ Núcleo: ${ }^{20} \mathrm{Ne}$} \\
$J^{\pi}$ & $E_{x}(\mathrm{MeV})$ & $\gamma_{\alpha}^{2}(\mathrm{keV})$ & $\theta_{\alpha}^{2}\left(10^{-3}\right)$ \\
\hline $0^{+}$ & 0.000 & 27 & 52 \\
$2^{+}$ & 1.634 & 29 & 56 \\
$4^{+}$ & 4.248 & 24 & 47 \\
$6^{+}$ & 8.778 & 20 & 38 \\
$8^{+}$ & 11.951 & 3 & 6
\end{tabular}

\begin{tabular}{cccc}
\multicolumn{4}{c}{ Núcleo: ${ }^{44} \mathrm{Ti}$} \\
$J^{\pi}$ & $E_{x}(\mathrm{MeV})$ & $\gamma_{\alpha}^{2}(\mathrm{keV})$ & $\theta_{\alpha}^{2}\left(10^{-3}\right)$ \\
\hline $0^{+}$ & 0.000 & 4.33 & 13.42 \\
$2^{+}$ & 1.083 & 4.60 & 14.28 \\
$4^{+}$ & 2.454 & 3.78 & 11.71 \\
$6^{+}$ & 4.015 & 2.24 & 6.94 \\
$\left(8^{+}\right)$ & 6.508 & 1.12 & 3.48 \\
$\left(10^{+}\right)$ & 7.671 & 0.22 & 0.69 \\
$\left(12^{+}\right)$ & 8.040 & 0.02 & 0.05
\end{tabular}

\begin{tabular}{cccc}
\multicolumn{4}{c}{ Núcleo: ${ }^{212} \mathrm{Po}$} \\
$J^{\pi}$ & $E_{x}(\mathrm{MeV})$ & $\gamma_{\alpha}^{2}(\mathrm{eV})$ & $\theta_{\alpha}^{2}\left(10^{-6}\right)$ \\
\hline $0^{+}$ & 0.000 & 57 & 398 \\
$2^{+}$ & 0.727 & 65 & 450 \\
$4^{+}$ & 1.133 & 52 & 361 \\
$6^{+}$ & 1.356 & 31 & 215 \\
$8^{+}$ & 1.476 & 14 & 98 \\
$10^{+}$ & 1.834 & 6 & 39 \\
\hline \hline
\end{tabular}

Figura 4.3. Seguindo o procedimento da Subseção 4.4.2, os raios de canal referentes aos núcleos ${ }^{20} \mathrm{Ne}$, ${ }^{44} \mathrm{Ti}$ e ${ }^{212} \mathrm{Po}$ foram definidos por meio da eq. (4.24), mesmo considerando a existência dos raios $a_{c}$ propostos na literatura para tais núcleos.

A Tabela 4.5 mostra que há diferentes ordens de grandeza de $\gamma_{\alpha}^{2}$ e $\theta_{\alpha}^{2}$ para cada estado $J^{+}$dos núcleos ${ }^{20} \mathrm{Ne},{ }^{44} \mathrm{Ti}$ e ${ }^{212} \mathrm{Po}$. Adicionando a esta comparação o núcleo 
${ }^{94} \mathrm{Mo}$, constata-se que este também difere dos anteriores na ordem de grandeza de $\gamma_{\alpha}^{2}$ e $\theta_{\alpha}^{2}$. O núcleo ${ }^{20} \mathrm{Ne}$ é aquele com as maiores ordens de grandeza observadas $\left(\gamma_{\alpha}^{2} \sim 30 \mathrm{keV}\right.$ e $\theta_{\alpha}^{2} \sim 5 \times 10^{-2}$ no estado fundamental $)$, seguido do ${ }^{44} \mathrm{Ti}\left(\gamma_{\alpha}^{2} \sim 4\right.$ $\mathrm{keV}$ e $\theta_{\alpha}^{2} \sim 1 \times 10^{-2}$ no e.f. $),{ }^{94} \mathrm{Mo}\left(\gamma_{\alpha}^{2} \sim 0.7 \mathrm{keV}\right.$ e $\theta_{\alpha}^{2} \sim 3 \times 10^{-3}$ no e.f. $)$ e ${ }^{212} \mathrm{Po}\left(\gamma_{\alpha}^{2} \sim 0.06 \mathrm{keV}\right.$ e $\theta_{\alpha}^{2} \sim 4 \times 10^{-4}$ no e.f. $)$. Este fato mostra que os núcleos de menor massa são aqueles com as maiores larguras- $\alpha$ reduzidas. A altura da barreira coulombiana em relação às energias dos estados $J^{+}$é um aspecto de grande influência nas larguras- $\alpha$ reduzidas, pois afeta o comportamento de $u_{N, L}(r)$ na região em torno do raio $a_{c}$. Os núcleos de maior massa possuem barreiras coulombianas mais altas que resultam numa queda mais abrupta de $u_{N, L}(r)$ na região próxima de $a_{c}$, afetando diretamente os valores de $\gamma_{\alpha}^{2}$ e $\theta_{\alpha}^{2}$.

Uma primeira interpretação das larguras- $\alpha$ reduzidas pode ser feita com referência nos núcleos leves. Comparando as ordens de grandeza de $\gamma_{\alpha}^{2}$ para os cinco núcleos da região do Mo e para ${ }^{20} \mathrm{Ne}$ e ${ }^{44} \mathrm{Ti}$, pode-se afirmar, em termos relativos, a existência de uma condição mais fraca de aglomeração- $\alpha$ para os núcleos da região do Mo em comparação aos núcleos leves.

Por outro lado, deve-se levar em conta os pequenos valores de $\theta_{\alpha}^{2}$ para ${ }^{20} \mathrm{Ne}$, ${ }^{44} \mathrm{Ti},{ }^{94} \mathrm{Mo}$ e ${ }^{212} \mathrm{Po}$. Apesar das diferenças de ordens de grandeza entre os valores de $\theta_{\alpha}^{2}$ para os quatro núcleos, nota-se que todos representam pequenas frações do limite de Wigner (o maior valor registrado refere-se ao estado $2^{+}$do ${ }^{20} \mathrm{Ne}$, que representa $\approx 6 \%$ do limite de Wigner). Portanto, as larguras- $\alpha$ reduzidas adimensionais indicam que as bandas de estado fundamental dos quatro núcleos possuem uma fraca característica de aglomeração- $\alpha$ em geral, sendo que esta condição se intensifica fortemente para os núcleos mais pesados. Tais resultados concordam com a análise de Michel, Reidemeister e Ohkubo [2] a respeito da banda de estado fundamental do ${ }^{44} \mathrm{Ti}$. Cálculos na literatura sobre os núcleos ${ }^{20} \mathrm{Ne}[13]$ e ${ }^{44} \mathrm{Ti}[2]$ descrevem a existência de bandas de energias superiores com características mais fortes de aglomeração- $\alpha$.

O comportamento fortemente decrescente de $\gamma_{\alpha}^{2}$ e $\theta_{\alpha}^{2}$ em relação a $J$ crescente repete-se para os núcleos ${ }^{20} \mathrm{Ne},{ }^{44} \mathrm{Ti}$ e ${ }^{212} \mathrm{Po}$. Isto mostra que a diminuição da intensidade de aglomeração- $\alpha$ para os estados de maior spin é um efeito presente nas estruturas $\alpha+$ caroço das diferentes regiões de massa. Este comportamento é compatível com o efeito antistretching já observado nas bandas de estado fundamental dos núcleos ${ }^{20} \mathrm{Ne}[1],{ }^{44} \mathrm{Ti}[2]$ e ${ }^{212} \mathrm{Po}$ [4] com diferentes formas de potencial $\alpha+$ caroço.

\subsection{Taxas de transição $B(E 2)$}

O modelo de cluster- $\alpha$ também é usado para o cálculo de taxas de transição entre os níveis do sistema. Neste trabalho, foi calculada a probabilidade de transição 
reduzida de quadrupolo elétrico entre níveis consecutivos das bandas de estado fundamental. No caso específico de sistemas onde o cluster e o caroço possuem spin nulo (sendo $J_{i}=L_{i}$ e $J_{f}=L_{f}$ ), a taxa $B(E 2)$ para transições $J \rightarrow J-2$ é calculada por $[3,8]$

$$
B(E 2 ; G, J \rightarrow J-2)=\frac{15}{8 \pi} \beta_{2}^{2} \frac{J(J-1)}{(2 J+1)(2 J-1)}\left\langle r_{J, J-2}^{2}\right\rangle^{2}
$$

onde

$$
\left\langle r_{J, J-2}^{2}\right\rangle=\int_{0}^{\infty} r^{2} u_{G, J}(r) u_{G, J-2}(r) d r
$$

e $\beta_{2}$ é mencionado como o fator de recuo, sendo dado por

$$
\beta_{2}=\frac{Z_{\alpha} A_{\mathrm{car}}^{2}+Z_{\mathrm{car}} A_{\alpha}^{2}}{\left(A_{\alpha}+A_{\mathrm{car}}\right)^{2}} .
$$

$\mathrm{Na}$ fórmula (4.26), as funções de onda radiais são identificadas através dos números quânticos $J$ e $G$.

Os valores de $B(E 2)$ referentes às bandas de estado fundamental dos núcleos da região do Mo encontram-se na Tabela 4.6. Observa-se que, com poucas exceções, os resultados teóricos apresentam a mesma ordem de grandeza dos valores experimentais e fornecem uma descrição satisfatória, considerando que não são usadas cargas efetivas nos cálculos e os parâmetros do potencial $\alpha+$ caroço não são ajustados com referência nos dados experimentais de $B(E 2)$. É conveniente lembrar que os dados experimentais de $B(E 2)$ são conhecidamente difíceis de serem reproduzidos com baixos erros relativos através do modelo de cluster- $\alpha$, principalmente nos núcleos meio-pesados e pesados [3, 4, 5].

Deve-se destacar, entre as poucas exceções citadas anteriormente, a diferença de ordens de grandeza entre os valores calculado e experimenatal de $B(E 2)$ na transição $8^{+} \rightarrow 6^{+}$do ${ }^{94}$ Mo. O valor experimenatal $B(E 2)_{\exp }=0.0049(8)$ W.u. para esta transição não é citado em cálculos anteriores sobre a estrutura de cluster- $\alpha$ no ${ }^{94} \mathrm{Mo}[3,4,5,6]$, provavelmente pelo fato da transição $8_{1}^{+}(2.956 \mathrm{MeV}) \rightarrow$ $6_{2}^{+}(2.423 \mathrm{MeV})$ ter sido confirmada experimentalmente apenas na referência mais atualizada sobre dados espectroscópicos do ${ }^{94} \mathrm{Mo}$ [40]. A mesma referência cita a existência de outra transição $8_{1}^{+}(2.956 \mathrm{MeV}) \rightarrow 6_{3}^{+}(2.872 \mathrm{MeV})$ cuja taxa de transição experimental é $B(E 2)_{\exp }=4.6(12)$ W.u., sendo este um valor muito mais favorável para a comparação com a taxa de transição calculada para $8^{+} \rightarrow 6^{+}$. Estes fatos indicam a possibilidade da transição $8_{1}^{+} \rightarrow 6_{3}^{+}$estar mais fortemente associada à estrutura de cluster- $\alpha$ do que a transição $8_{1}^{+} \rightarrow 6_{2}^{+}$usada na comparação com os cálculos. Porém, se o nível experimental $6_{3}^{+}$fosse considerado um membro da banda $G=16$ do ${ }^{94} \mathrm{Mo}$, haveria uma grande incompatibilidade com o espectro teórico produzido pelo potencial $\alpha+$ caroço com $V_{0}=220 \mathrm{MeV}$, pois 
Tabela 4.6: Taxas de transição $B(E 2)_{\text {calc }}$ calculadas para as bandas de estado fundamental dos núcleos ${ }^{90} \mathrm{Sr},{ }^{92} \mathrm{Zr},{ }^{94} \mathrm{Mo},{ }^{96} \mathrm{Ru}$ e ${ }^{98} \mathrm{Pd}$ em comparação com dados experimentais $\left(B(E 2)_{\exp }\right)$ disponíveis. Todos os valores são dados na unidade Weisskopf (W.u.). As taxas $B(E 2)_{\text {calc }}$ foram obtidas sem o uso de cargas efetivas. As referências dos dados experimentais são indicadas na própria tabela.

\begin{tabular}{ccc}
\hline \hline & $\begin{array}{c}\text { Núcleo: }{ }^{90} \mathrm{Sr} \\
B(E 2)_{\text {calc }} \\
\text { (W.u.) }\end{array}$ & $\begin{array}{c}B(E 2)_{\exp }[37] \\
\text { (W.u.) }\end{array}$ \\
\hline $2^{+} \rightarrow 0^{+}$ & 5.569 & $8.5(24)$ \\
$4^{+} \rightarrow 2^{+}$ & 7.635 & $5.2(9)$
\end{tabular}

Núcleo: ${ }^{92} \mathrm{Zr}$

\begin{tabular}{ccc} 
Transição & $\begin{array}{c}B(E 2)_{\text {calc }} \\
\text { (W.u.) }\end{array}$ & $\begin{array}{c}B(E 2)_{\exp }[38] \\
\text { (W.u.) }\end{array}$ \\
\hline $2^{+} \rightarrow 0^{+}$ & 5.623 & $6.4(6)$ \\
$4^{+} \rightarrow 2^{+}$ & 7.661 & $4.04(12)$ \\
$6^{+} \rightarrow 4^{+}$ & 7.727 & \\
$\left(8^{+}\right) \rightarrow 6^{+}$ & 6.610 & $3.59(22)$ \\
$\left(10^{+}\right) \rightarrow\left(8^{+}\right)$ & 5.268 & \\
$\left(12^{+}\right) \rightarrow\left(10^{+}\right)$ & 3.569 & $>0.056$ \\
$\left(14^{+}\right) \rightarrow\left(12^{+}\right)$ & 1.859 &
\end{tabular}

Núcleo: ${ }^{94} \mathrm{Mo}$

\begin{tabular}{ccc} 
Transição & $\begin{array}{c}B(E 2)_{\text {calc }} \\
\text { (W.u.) }\end{array}$ & $\begin{array}{c}B(E 2)_{\exp }[40] \\
\text { (W.u.) }\end{array}$ \\
\hline $2^{+} \rightarrow 0^{+}$ & 7.757 & $16.0(4)$ \\
$4^{+} \rightarrow 2^{+}$ & 10.738 & $26(4)$ \\
$6^{+} \rightarrow 4^{+}$ & 10.898 & \\
$8^{+} \rightarrow 6^{+}$ & 9.798 & $0.0049(8)$ \\
$\left(10^{+}\right) \rightarrow 8^{+}$ & 8.303 & \\
$\left(12^{+}\right) \rightarrow\left(10^{+}\right)$ & 6.237 & \\
\hline \hline
\end{tabular}


Tabela 4.6 (continuação)

\begin{tabular}{|c|c|c|}
\hline Transição & $\begin{array}{c}\text { Núcleo: }{ }^{96} \mathrm{Ru} \\
B(E 2)_{\text {calc }} \\
\text { (W.u.) }\end{array}$ & $\begin{array}{c}B(E 2)_{\exp }[42] \\
\text { (W.u.) }\end{array}$ \\
\hline $2^{+} \rightarrow 0^{+}$ & 7.539 & $18.4(4)$ \\
\hline $4^{+} \rightarrow 2^{+}$ & 10.430 & $20.7(15)$ \\
\hline $6^{+} \rightarrow 4^{+}$ & 10.512 & $14(5)$ \\
\hline $8^{+} \rightarrow 6^{+}$ & 9.584 & $6.0(22)$ \\
\hline $10^{+} \rightarrow 8^{+}$ & 8.098 & $12.7(15)$ \\
\hline $12^{+} \rightarrow 10^{+}$ & 6.190 & 13.1(19) \\
\hline $14^{+} \rightarrow 12^{+}$ & 4.341 & $2.63(23)$ \\
\hline $16^{+} \rightarrow 14^{+}$ & 2.186 & $>11$ \\
\hline Transição & $\begin{array}{c}\text { Núcleo: }{ }^{98} \mathrm{Pd} \\
B(E 2)_{\text {calc }} \\
\text { (W.u.) }\end{array}$ & $\begin{array}{c}B(E 2)_{\exp } \\
\text { (W.u.) }\end{array}$ \\
\hline $2^{+} \rightarrow 0^{+}$ & 7.364 & \\
\hline $4^{+} \rightarrow 2^{+}$ & 10.195 & \\
\hline$\left(6^{+}\right) \rightarrow 4^{+}$ & 10.253 & (Sem dados \\
\hline$\left(8^{+}\right) \rightarrow\left(6^{+}\right)$ & 9.315 & disponíveis.) \\
\hline$\left(10^{+}\right) \rightarrow\left(8^{+}\right)$ & 7.882 & {$[43]$} \\
\hline$\left(12^{+}\right) \rightarrow\left(10^{+}\right)$ & 6.097 & \\
\hline$\left(14^{+}\right) \rightarrow\left(12^{+}\right)$ & 4.257 & \\
\hline$\left(16^{+}\right) \rightarrow\left(14^{+}\right)$ & 2.179 & \\
\hline
\end{tabular}

o intervalo aproximado de energia entre $8_{1}^{+}$e $6_{3}^{+}$é de apenas $83 \mathrm{keV}$. Além do referido problema, não há registro na Ref. [40] sobre uma transição $E 2$ entre $6_{3}^{+}$e $4_{1}^{+}(1.574 \mathrm{MeV})$, o que também desfavorece a inclusão de $6_{3}^{+}$como membro da banda de estado fundamental. Portanto, novos dados experimentais sobre transições- $\gamma$ e taxas de transição podem contribuir para a elucidação desta questão.

Observando as transições $2^{+} \rightarrow 0^{+}$e $4^{+} \rightarrow 2^{+}$dos núcleos ${ }^{90} \mathrm{Sr},{ }^{92} \mathrm{Zr},{ }^{94} \mathrm{Mo} \mathrm{e}$ ${ }^{96} \mathrm{Ru}$, nota-se que as taxas $B(E 2)$ exp para os núcleos ${ }^{94}$ Mo e ${ }^{96} \mathrm{Ru}$ são $\approx 2$ a $6 \times$ maiores do que as taxas $B(E 2) \exp$ dos núcleos ${ }^{90} \mathrm{Sr}$ e ${ }^{92} \mathrm{Zr}$. Este fato mostra que a escolha dos números quânticos $G=14$ para ${ }^{90} \mathrm{Sr}$ e ${ }^{92} \mathrm{Zr}$ e $G=16$ para ${ }^{94} \mathrm{Mo}$ e ${ }^{96} \mathrm{Ru}$ é favorável para uma melhor descrição das taxas de transição experimentais. As funções $u_{G, J}(r)$ produzidas com $G=16$ são mais extensas ao longo de $r$ do que as funções $u_{G, J}(r) \operatorname{com} G=14$, o que resulta em taxas de transição $B(E 2)_{\text {calc }}$ 
mais altas para ${ }^{94} \mathrm{Mo} \mathrm{e}{ }^{96} \mathrm{Ru}$ e mais baixas para ${ }^{90} \mathrm{Sr}$ e ${ }^{92} \mathrm{Zr}$, como pode ser visto na Tabela 4.6. Portanto, mostra-se uma coerência entre os números quânticos $G$ aplicados e os dados experimentais de $B(E 2)$.

Uma vantagem do modelo de cluster- $\alpha$ em relação aos cálculos de modelo de camadas é a capacidade de reprodução das ordens de grandeza da maior parte dos dados experimentais de $B(E 2)$ sem o uso de cargas efetivas. Como exemplo, a Ref. [53] mostra um cálculo detalhado usando o modelo de camadas para vários núcleos de $A=92-98$, onde são considerados prótons e nêutrons de valência ocupando vários orbitais. Na referência citada, constatamos o uso de cargas efetivas acentuadas $\left(e_{\mathrm{ef}}^{p}=1.5 e\right.$ e $\left.e_{\mathrm{ef}}^{n}=0.5 e\right)$ no cálculo das taxas $B(E 2)$ nos núcleos ${ }^{92} \mathrm{Zr},{ }^{94} \mathrm{Mo},{ }^{96} \mathrm{Ru}$ e ${ }^{98} \mathrm{Pd}$, sendo que a maioria dos resultados obtidos são ainda mais afastados dos dados experimentais em comparação com os valores de $B(E 2)$ calculados neste trabalho. 


\section{Capítulo 5}

\section{Procura por bandas de paridade negativa nos sistemas $\alpha+$ caroço}

Este capítulo mostra as bandas de paridade negativa calculadas para os sistemas $\alpha+$ caroço dos núcleos ${ }^{92} \mathrm{Zr},{ }^{94} \mathrm{Mo},{ }^{96} \mathrm{Ru}$ e ${ }^{98} \mathrm{Pd}$ e descreve os procedimentos específicos aplicados para a determinação de tais bandas. Os resultados são discutidos em comparação com dados experimentais disponíveis.

\subsection{Exemplos na literatura sobre bandas de paridade negativa}

Os potenciais $\alpha+$ caroço podem gerar bandas de níveis superiores conforme a regra $G \geq G_{1}$, onde $G_{1}$ é o número quântico global associado à banda de menor energia do sistema $\alpha+$ caroço. No caso dos núcleos estudados neste trabalho, $G_{1}$ está associado à banda de estado fundamental, a qual possui níveis de paridade positiva. O número quântico seguinte $\left(G_{2}=G_{1}+1\right)$ está associado à primeira banda de paridade negativa que se inicia a partir de um estado excitado do sistema. Dependendo da forma e parâmetros do potencial $\alpha+$ caroço, mais bandas superiores podem surgir.

Várias referências da literatura (exemplos nas Refs. [1, 2, 14, 54]) discutem a existência de bandas de paridade negativa associadas à estrutura $\alpha+$ caroço em diferentes regiões de massa. Contudo, um problema mencionado em muitos trabalhos é a falta de níveis experimentais com spin e paridade definidos que sirvam como referência para cálculos envolvendo as bandas excitadas. Entre os principais núcleos onde a estrutura $\alpha+$ caroço é estudada, os leves ${ }^{16} \mathrm{O}$ e ${ }^{20} \mathrm{Ne}$ possuem vários níveis experimentais de paridade negativa que constituem bandas associadas às estruturas $\alpha+{ }^{12} \mathrm{C}$ e $\alpha+{ }^{16} \mathrm{O}$, respectivamente [1, 14]. As cabeças de banda ${ }^{1}$ de

\footnotetext{
${ }^{1} \mathrm{O}$ termo "cabeça de banda" é empregado como o equivalente ao termo band head das publi-
} 
paridade negativa para os núcleos ${ }^{16} \mathrm{O}$ e ${ }^{20} \mathrm{Ne}$ encontram-se em $E_{x} \approx 9.6 \mathrm{MeV}$ e $E_{x} \approx 5.8 \mathrm{MeV}$, respectivamente.

As bandas de paridade negativa dos sistemas $\alpha+{ }^{36} \mathrm{Ar}$ e $\alpha+{ }^{40} \mathrm{Ca}$, os quais associam-se aos núcleos ${ }^{40} \mathrm{Ca}$ e ${ }^{44} \mathrm{Ti}$, foram calculadas em diferentes trabalhos da literatura $[2,14,22,35,36]$. O estudo detalhado de T. Yamaya et al. [54] sobre as reações de transferência ${ }^{36} \mathrm{Ar}\left({ }^{6} \mathrm{Li}, d\right){ }^{40} \mathrm{Ca}$ e ${ }^{40} \mathrm{Ca}\left({ }^{6} \mathrm{Li}, d\right){ }^{44} \mathrm{Ti}$ confirmou a existência de bandas experimentais de paridade negativa para os dois núcleos. De acordo com o mesmo estudo, as cabeças de banda de paridade negativa para os núcleos ${ }^{40} \mathrm{Ca}$ e ${ }^{44} \mathrm{Ti}$ encontram-se em $E_{x} \approx 8.6 \mathrm{MeV}$ e $E_{x} \approx 6.2 \mathrm{MeV}$, respectivamente, mas deve ser levada em conta a observação experimental de componentes dos estados de estrutura- $\alpha$ distribuídos em diferentes níveis dos espectros dos dois núcleos.

A respeito da estrutura $\alpha+$ caroço no ${ }^{94} \mathrm{Mo}$, há trabalhos na literatura $[4,6,17]$ que citam estimativas sobre a localização da banda de paridade negativa do sistema $\alpha+{ }^{90} \mathrm{Zr}$. Tais referências indicam que a cabeça de banda de paridade negativa do sistema $\alpha+{ }^{90} \mathrm{Zr}$ deve localizar-se em $E_{x} \approx 6$ a $7 \mathrm{MeV}$, contudo, sem mostrar comparações com níveis de energia experimentais do ${ }^{94} \mathrm{Mo}$.

Os trabalhos que tratam da estrutura $\alpha+$ caroço no ${ }^{212} \mathrm{Po}[3,4,7]$ não mostram cálculos sobre uma possível banda de paridade negativa, pois não há níveis do espectro experimental do ${ }^{212} \mathrm{Po}[46]$ que permitam uma comparação com esta banda teórica.

\subsection{Bandas de paridade negativa nos núcleos da região do Molibdênio}

\subsubsection{Aspectos específicos do cálculo das bandas de paridade negativa}

A primeira banda de paridade negativa de um sistema cluster + caroço, no qual cluster e caroço possuem spins nulos, é caracterizada pelo número quântico global $G_{2}=G_{1}+1$, onde $G_{1}$ é o número associado à primeira banda de paridade positiva. Desta forma, os números quânticos $G$ associados às bandas de paridade negativa dos núcleos estudados neste trabalho são:

$$
\begin{gathered}
G=15 \text { para }{ }^{90} \mathrm{Sr} \mathrm{e}{ }^{92} \mathrm{Zr}, \\
G=17 \text { para }{ }^{94} \mathrm{Mo},{ }^{96} \mathrm{Ru} \mathrm{e}{ }^{98} \mathrm{Pd} .
\end{gathered}
$$

cações em inglês. Este termo é usado para definir o primeiro estado (o de mais baixa energia) de uma banda de níveis. 
A respeito dos parâmetros do potencial $\alpha+$ caroço, foi escolhido o procedimento onde $R, a$ e $b$ são mantidos com os mesmos valores aplicados para a descrição geral das bandas de estado fundamental dos cinco núcleos (ver Seção 3.4), enquanto a profundidade $V_{0}$ foi reajustada com precisão de $1 \mathrm{MeV}$ para a melhor reprodução possível dos estados experimentais $5^{-}$de cada núcleo. O baixo grau de precisão no reajuste de $V_{0}$ foi adotado para estabelecer uma correspondência com a precisão do valor $V_{0}=220 \mathrm{MeV}$ aplicado nas bandas de estado fundamental.

\subsubsection{Procedimento específico para a determinação de níveis de energia ressonantes}

Como as bandas de paridade negativa iniciam-se a partir de estados excitados, é natural que os níveis ressonantes de tais bandas estejam localizados mais acima do limiar $\alpha+$ caroço do que os estados ressonantes das bandas de estado fundamental. Os níveis que se encontram muitos $\mathrm{MeV}$ acima do limiar, isto é, no intervalo de energia onde a barreira de potencial efetivo é menos extensa, podem ser tratados por um método específico para estados ressonantes. Adaptando o processo de varredura de energia usado originalmente para a determinação de estados ligados ou quase-ligados (ver Seção 3.2), pode-se substituir a condição inicial de poço quadrado em $r_{0} \gg 0$ (na Região II) pela forma

$$
u_{\mathrm{II}}\left(r_{0}\right)=B \sin \left[k r_{0}-\gamma \ln \left(2 k r_{0}\right)-\frac{1}{2} L \pi+\sigma_{L}+\delta_{L}\right],
$$

baseada na forma assintótica das funções de Coulomb esféricas, onde $\sigma_{L}$ é o deslocamento de fase coulombiano, $\delta_{L}=\pi / 2$ é o deslocamento de fase resultante do potencial nuclear, $k=\mu v / \hbar$ é o número de onda associado à massa reduzida do sistema e $\gamma$ é dado por

$$
\gamma=\left(\frac{e^{2}}{4 \pi \varepsilon_{0}}\right) \frac{Z_{\alpha} Z_{\mathrm{car}}}{\hbar v}
$$

sendo $v$ a velocidade relativa entre o caroço e o cluster- $\alpha$ no infinito. Portanto, a condição inicial (5.1) baseia-se no tipo de função de onda que representa o espalhamento do cluster- $\alpha$ no potencial de interação $\alpha+$ caroço. O valor fixo $\delta_{L}=\pi / 2$ é aquele que corresponde à energia de ressonância do estado acima do limiar. O coeficiente $B$ deve ser calculado de tal forma que seja mantida a continuidade da solução $u(r)$ da equação radial (3.4) no raio $r_{\mathrm{f}}$ (ver Seção 3.2). Conforme a sugestão da Ref. [33], o raio $r_{0}$ foi definido através da fórmula

$$
r_{0}=\frac{60\left[L(L+1)+\gamma^{2}\right]}{k}
$$


produzida com referência na condição assintótica das funções de Coulomb esféricas sem a presença de interações de curto alcance [55].

Com a adaptação baseada na eq. (5.1), o processo de varredura de energia é executado da mesma forma já descrita na Seção 3.2, contudo, visando calcular as energias de ressonância acima do limiar. Neste trabalho, tal adaptação foi aplicada somente para energias onde a barreira de potencial efetivo se estende até um raio $\lesssim 30 \mathrm{fm}^{2}{ }^{2}$ Esta forma de varredura de energia também foi aplicada para o cálculo dos níveis ressonantes da banda de estado fundamental do sistema $\alpha+{ }^{208} \mathrm{~Pb}$, conforme as discussões da Subseção 4.1.3.

\subsubsection{Resultados obtidos para as bandas de paridade negativa}

As bandas de paridade negativa calculadas para os núcleos ${ }^{92} \mathrm{Zr},{ }^{94} \mathrm{Mo},{ }^{96} \mathrm{Ru}$ e ${ }^{98} \mathrm{Pd}$ são apresentadas nas Figuras entre 5.1 e 5.4 em comparação com níveis de energia experimentais disponíveis. Com relação ao núcleo ${ }^{90} \mathrm{Sr}$, a sua banda de paridade negativa não foi calculada devido à falta de níveis experimentais suficientes para uma comparação satisfatória. Verifica-se que as quatro bandas calculadas apresentam as mesmas características observadas nas bandas de estado fundamental, onde há um comportamento quase-rotacional nos primeiros níveis e o efeito de compressão dos últimos níveis. Infelizmente, há poucos níveis experimentais do ${ }^{92} \mathrm{Zr}$ para um julgamento consistente da respectiva banda teórica, e a maior parte dos níveis experimentais de paridade negativa dos quatro núcleos não apresenta spin e paridade confirmados. Outro fato relacionado às bandas experimentais é a ausência de níveis $1^{-}$e $3^{-}$para os quatro núcleos (detalhes na Seção 5.3). Apesar dos dados experimentais limitados, pode-se afirmar que a descrição geral dos espaçamentos entre os níveis experimentais é satisfatória, levando-se em conta que somente o parâmetro $V_{0}$ foi reajustado para a descrição das bandas de paridade negativa. Especificamente no caso do núcleo ${ }^{96} \mathrm{Ru}$, o espaçamento entre os níveis experimentais $15^{(-)}$e $17^{(-)}$é reproduzido grosseiramente devido ao efeito de compressão nos últimos níveis da banda teórica.

Os valores de $V_{0}$ aplicados para a descrição das bandas de paridade negativa são:

$$
\begin{gathered}
V_{0}=239 \mathrm{MeV} \text { para }{ }^{92} \mathrm{Zr} \\
V_{0}=237 \mathrm{MeV} \text { para }{ }^{94} \mathrm{Mo},{ }^{96} \mathrm{Ru} \mathrm{e}{ }^{98} \mathrm{Pd}
\end{gathered}
$$

\footnotetext{
${ }^{2}$ Para barreiras que se estendem além de $30 \mathrm{fm}$, a aproximação de estado ligado descrita na Seção 3.3 ainda é bastante apropriada para os núcleos estudados neste trabalho, como se demonstra na Subseção 5.2.3.
} 


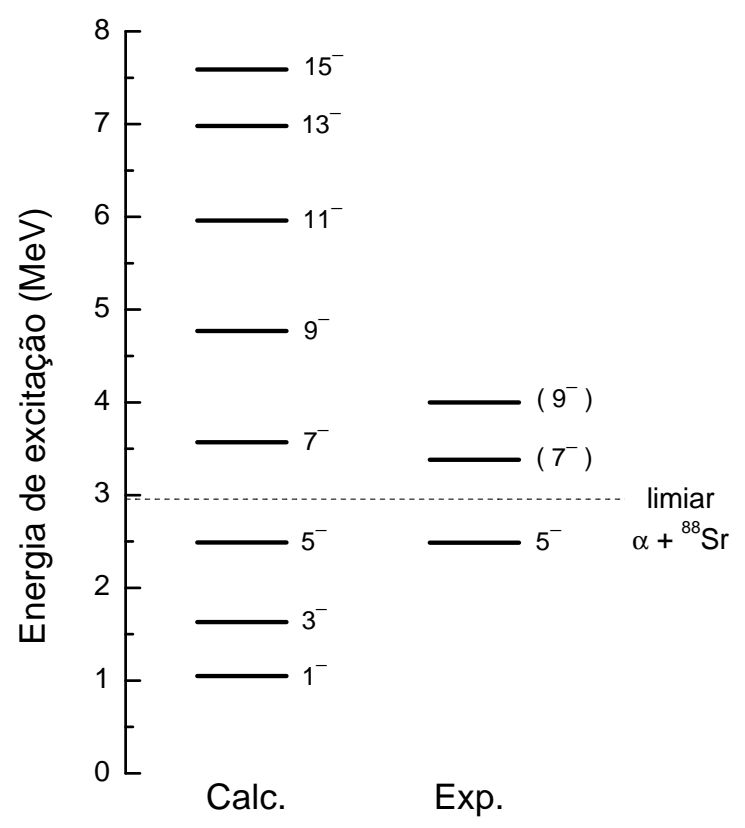

Figura 5.1: Comparação entre a banda de paridade negativa $(G=15)$ calculada para o sistema $\alpha+{ }^{88} \mathrm{Sr}$ e níveis de energia experimentais [38] do ${ }^{92} \mathrm{Zr}$. Os valores aplicados aos parâmetros do potencial $\alpha+$ caroço são discutidos nesta seção.

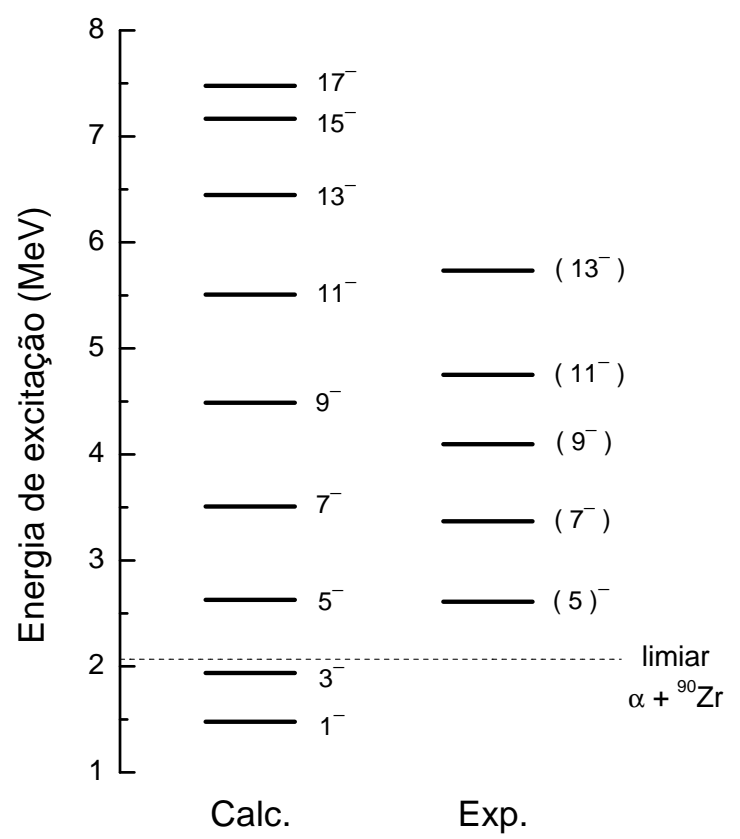

Figura 5.2: Comparação entre a banda de paridade negativa $(G=17)$ calculada para o sistema $\alpha+{ }^{90} \mathrm{Zr}$ e níveis de energia experimentais [40] do ${ }^{94}$ Mo. Os valores aplicados aos parâmetros do potencial $\alpha+$ caroço são discutidos nesta seção. 


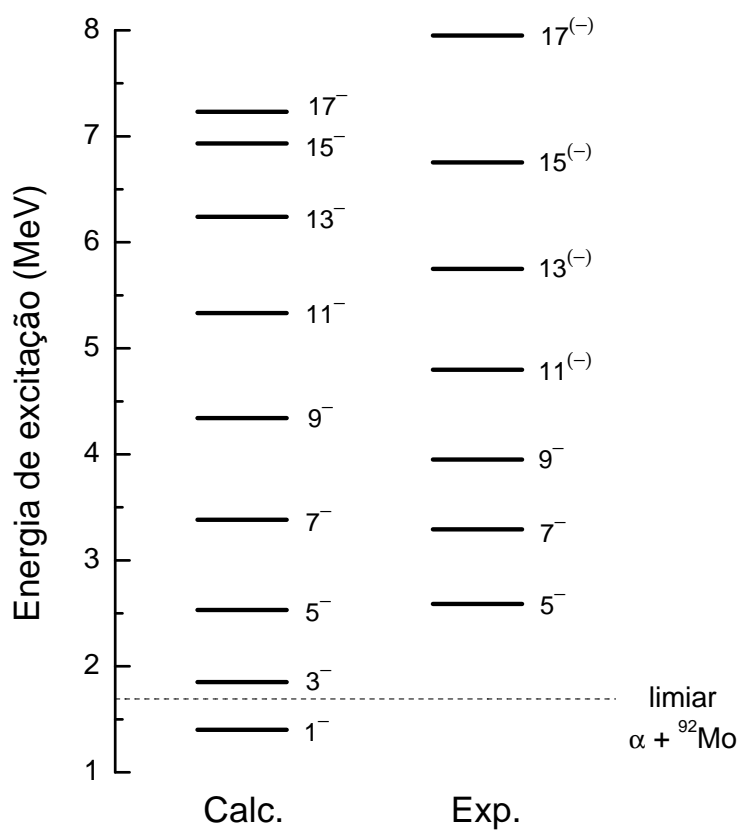

Figura 5.3: Comparação entre a banda de paridade negativa $(G=17)$ calculada para o sistema $\alpha+{ }^{92}$ Mo e níveis de energia experimentais [42] do ${ }^{96} \mathrm{Ru}$. Os valores aplicados aos parâmetros do potencial $\alpha+$ caroço são discutidos nesta seção.

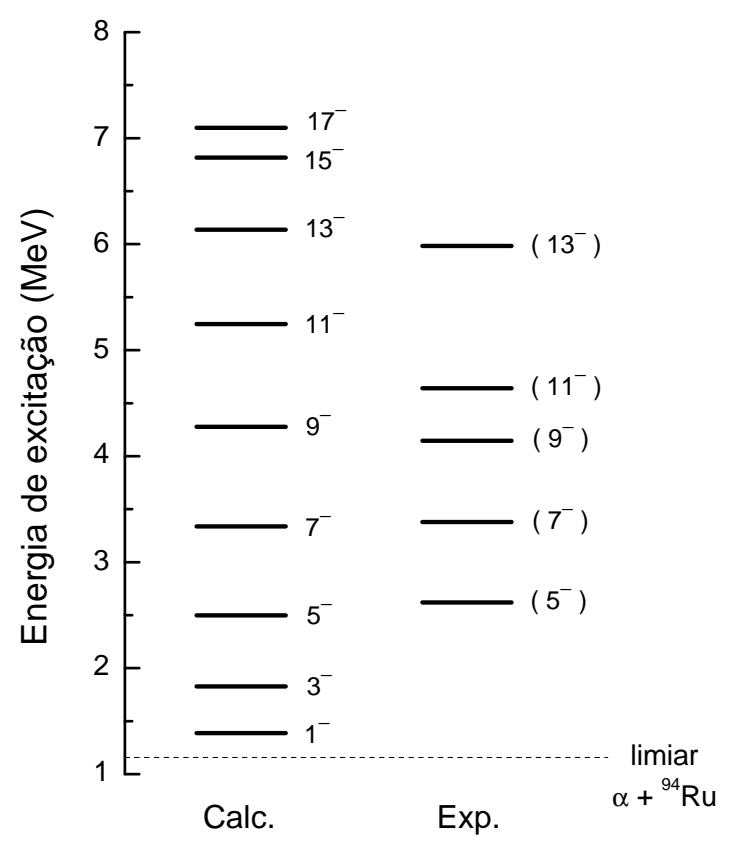

Figura 5.4: Comparação entre a banda de paridade negativa $(G=17)$ calculada para o sistema $\alpha+{ }^{94} \mathrm{Ru}$ e níveis de energia experimentais [43] do ${ }^{98} \mathrm{Pd}$. Os valores aplicados aos parâmetros do potencial $\alpha+$ caroço são discutidos nesta seção. 
Os referidos valores são, respectivamente, $\approx 8.6 \% \mathrm{e} \approx 7.7 \%$ maiores do que a profundidade $V_{0}=220 \mathrm{MeV}$, o que demonstra uma baixa variação relativa à profundidade usada para as bandas de estado fundamental. A dependência fraca, mas não desprezível, do potencial $\alpha+$ caroço em relação à paridade é um fato verificado em outros núcleos de diferentes regiões de massa $[1,56]$. A Ref. [1] mostra que os potenciais nucleares $\alpha+$ caroço do tipo folding para os núcleos ${ }^{16} \mathrm{O}$ e ${ }^{20} \mathrm{Ne}$ possuem um parâmetro de intensidade $\bar{f}$ dependente da paridade ${ }^{3}$, sendo que os valores de $\bar{f}$ para as bandas de paridade negativa dos dois núcleos $(G=9)$ são, respectivamente, $\approx 8.8 \%$ e $\approx 7.1 \%$ maiores do que os valores de $\bar{f}$ aplicados às suas bandas de paridade positiva $(G=8)$ correspondentes.

A proximidade entre os valores de $V_{0}$ aplicados às bandas de paridade negativa demonstra que há uma tendência de padronização deste parâmetro para as mesmas bandas. Indica-se que $V_{0}=237 \mathrm{MeV}$ é um valor adequado para a descrição geral das bandas associadas a $G=17$; contudo, se a banda $G=15$ do ${ }^{92} \mathrm{Zr}$ é incluída nesta avaliação, indica-se que a profundidade mais conveniente para a descrição geral dos quatro núcleos deve estar próxima do valor médio $V_{0}=237.5 \mathrm{MeV}$ referente às quatro profundidades ajustadas.

O procedimento para a determinação de níveis ressonantes da Subseção 5.2.2 foi aplicado somente para os estados teóricos $17^{-}$dos núcleos ${ }^{94} \mathrm{Mo},{ }^{96} \mathrm{Ru}$ e ${ }^{98} \mathrm{Pd}$, onde o limite externo da barreira de potencial efetivo é próximo de $r=30 \mathrm{fm}$. Verificou-se que o processo de varredura para níveis ressonantes produz resultados iguais aos obtidos por meio da aproximação de estado ligado (ver Seção 3.3), considerando o passo de energia $\Delta E=0.01 \mathrm{MeV}$ aplicado em ambos os casos. Este fato mostra que as bandas de paridade negativa dos quatro núcleos são constituídas de estados quase-ligados nos quais o comportamento ressonante é desprezível. Para a confirmação desta característica, as larguras- $\alpha$ totais $\Gamma_{\alpha}$ dos últimos níveis teóricos das bandas de paridade negativa $\left(15^{-}\right.$para ${ }^{92} \mathrm{Zr}$ e $17^{-}$para ${ }^{94} \mathrm{Mo},{ }^{96} \mathrm{Ru}$ e $\left.{ }^{98} \mathrm{Pd}\right)$ foram calculadas através da fórmula semiclássica sugerida na Ref. [7], com um fator de pré-formação $P=1$ referente ao cluster- $\alpha$. Todos os valores calculados para $\Gamma_{\alpha}$ encontram-se na ordem de grandeza de $10^{-25} \mathrm{MeV}$, confirmando que os estados de paridade negativa acima do limiar $\alpha+$ caroço são praticamente ligados.

\subsection{Aspectos referentes aos níveis de energia experimentais}

Os níveis experimentais de paridade negativa comparados às bandas teóricas foram escolhidos conforme o procedimento já usado para as bandas de estado fun-

\footnotetext{
${ }^{3} \mathrm{O}$ parâmetro de intensidade $\bar{f}$ do potencial folding exerce uma função similar a do parâmetro $V_{0}$ usado no potencial $\alpha+$ caroço deste trabalho.
} 
damental, isto é, selecionando níveis experimentais de energia mais baixa possível, contudo, que estejam conectados por transições do tipo $E 2$ de maior intensidade. Também foi considerado desejável a escolha de níveis populados em reações que indicam ou podem indicar a presença da estrutura de cluster- $\alpha$ nos mesmos níveis. A seguir, são discutidos os detalhes referentes às reações onde os níveis escolhidos são populados, além de outros aspectos experimentais.

Com relação aos níveis experimentais do ${ }^{92} \mathrm{Zr}$, verifica-se que há somente correspondentes para os níveis teóricos $5^{-}, 7^{-}$e $9^{-}$, porém, apenas o nível experimental $5^{-}\left(E_{x}=2.48602 \mathrm{MeV}\right)$ possui spin e paridade confirmados. O espectro geral do ${ }^{92} \mathrm{Zr}$ [38] mostra que há um nível experimental $3^{-}$em $E_{x}=2.33972 \mathrm{MeV}$, contudo, este nível não foi incluído na comparação com a banda teórica, pois não há uma transição- $\gamma$ observada entre o nível experimenatal $5^{-}\left(E_{x}=2.48602 \mathrm{MeV}\right)$ e o referido estado $3^{-}$. As referências [38] e [39] afirmam que os três níveis experimentais comparados à banda calculada são populados na reação ${ }^{8} \mathrm{Sr}\left({ }^{7} \mathrm{Li}, 2 n p \gamma\right){ }^{92} \mathrm{Zr}$, a qual configura uma forma de transferência- $\alpha$ no ${ }^{88} \mathrm{Sr}$. Assim como nos níveis experimentais de paridade positiva (ver Seção 3.6), este fato pode ser interpretado como uma indicação da estrutura de cluster- $\alpha$ nestes estados. É importante mencionar que o estado experimental $3^{-}$citado anteriormente não é populado na mesma reação.

Discutindo os níveis experimentais do ${ }^{94} \mathrm{Mo}$, verifica-se que há correspondentes para os níveis teóricos entre $5^{-}$e $13^{-}$, porém, nenhum destes níveis experimentais possui spin e paridade confirmados. O espectro geral do ${ }^{94} \mathrm{Mo}$ [40] mostra que há níveis experimentais $\left(3^{-}\right),\left(15^{-}\right)$e $\left(17^{-}\right)$que respeitam a sequência de spin e energia crescentes exigida para a banda experimental, contudo, tais níveis não foram incluídos na comparação com a banda teórica, pois não há transições- $\gamma$ que estabeleçam uma conecção com os demais níveis experimentais selecionados para a comparação. Ao contrário dos níveis da banda de estado fundamental do ${ }^{94} \mathrm{Mo}$, não há indicações no espectro geral de que os estados experimentais de paridade negativa sejam populados em reações de transferência- $\alpha$, decaimento- $\alpha$ ou outra reação relacionada a este contexto.

Com relação aos níveis experimentais do ${ }^{96} \mathrm{Ru}$, verifica-se que há correspondentes para os níveis teóricos entre $5^{-}$e $17^{-}$, contudo, os níveis experimentais com spins entre $J=11$ e $J=17$ não possuem a paridade negativa confirmada. Não há indicações no espectro geral do ${ }^{96} \mathrm{Ru}[42]$ sobre estados experimentais de paridade negativa populados em reações de transferência- $\alpha$, decaimento- $\alpha$ ou outra reação relacionada a este contexto.

A respeito dos níveis experimentais do ${ }^{98} \mathrm{Pd}$, verifica-se que há correspondentes para os níveis teóricos entre $5^{-}$e $13^{-}$, contudo, nenhum destes níveis experimentais possui spin e paridade confirmados. Não há indicações no espectro geral do ${ }^{98} \mathrm{Pd}$ [43] sobre estados experimentais de paridade negativa populados em reações de 
transferência- $\alpha$, decaimento- $\alpha$ ou outra reação relacionada a este contexto. 


\section{Conclusões e perspectivas}

O presente trabalho mostrou que o modelo de cluster- $\alpha$ com Abordagem de Potencial Local produz uma boa descrição geral das bandas do estado fundamental dos núcleos ${ }^{90} \mathrm{Sr},{ }^{92} \mathrm{Zr},{ }^{94} \mathrm{Mo},{ }^{96} \mathrm{Ru}$ e ${ }^{98} \mathrm{Pd}$ por meio do potencial fenomenológico $\alpha+$ caroço de Buck, Merchant e Perez [3] com apenas 1 parâmetro variável, e fornece resultados satisfatórios para as taxas de transição $B(E 2)$ sem a introdução de cargas efetivas. O modelo também permitiu o cálculo de outras propriedades relacionadas à estrutura de cluster- $\alpha$ nas bandas do estado fundamental e a identificação de bandas de paridade negativa associadas a esta estrutura. Visando uma análise mais abrangente das propriedades dos cinco núcleos da região do Mo, os núcleos ${ }^{20} \mathrm{Ne},{ }^{44} \mathrm{Ti}$ e ${ }^{212}$ Po também foram discutidos em algumas de suas propriedades na perspectiva do modelo de cluster- $\alpha$. Uma síntese dos resultados alcançados nos diferentes tópicos é apresentada a seguir:

1. O critério de comparação de $Q_{\alpha} / A_{T}$ corrobora as afirmações da literatura de que os núcleos ${ }^{20} \mathrm{Ne},{ }^{44} \mathrm{Ti},{ }^{94} \mathrm{Mo}$ e ${ }^{212} \mathrm{Po}$ são os preferenciais para a aglomeração- $\alpha$ em suas respectivas regiões. Com relação aos núcleos da região do Mo, o critério de comparação de $Q_{\alpha} / A_{T}$ mostra que os núcleos ${ }^{90} \mathrm{Sr},{ }^{92} \mathrm{Zr}$, ${ }^{94} \mathrm{Mo},{ }^{96} \mathrm{Ru}$ e ${ }^{98} \mathrm{Pd}$ são os preferenciais para a aglomeração- $\alpha$ quando são comparados aos seus respectivos conjuntos de isótopos par-par e isóbaros par-par simultaneamente. Estes resultados indicam que os núcleos mais favoráveis para a estrutura $\alpha+$ caroço na região de massa do Mo são aqueles com $N=52$, isto é, os núcleos cujos caroços nucleares possuem o número mágico de nêutrons $N=50$.

2. É mostrado que o potencial $\alpha+$ caroço na forma W.S. + W.S. ${ }^{3}$ fornece uma boa reprodução geral dos espaçamentos entre os níveis experimentais das bandas de estado fundamental dos cinco núcleos da região do Mo, considerando que apenas o parâmetro $R$ é ajustado para cada núcleo e que o conjunto de parâmetros fixos $\left(V_{0}, a, b\right)$ é usado para a descrição da mesma estrutura em núcleos de diferentes regiões de massa. Revela-se que o potencial nuclear W.S. + W.S. ${ }^{3}$ associado ao conjunto fixo $\left(V_{0}, a, b\right)$ pode ser aplicado satisfatoriamente na descrição das bandas de outros núcleos além daqueles 
com a configuração $\alpha+$ (caroço de dupla camada fechada).

3. A pesquisa no espectro geral do ${ }^{92} \mathrm{Zr}$ [38] e na Ref. [39] mostra que, com exceção do estado $\left(14^{+}\right)$, todos os níveis experimentais da banda de estado fundamental são populados na reação ${ }^{88} \mathrm{Sr}\left({ }^{7} \mathrm{Li}, 2 n p \gamma\right){ }^{92} \mathrm{Zr}$, a qual configura uma forma de transferência- $\alpha$ no ${ }^{88} \mathrm{Sr}$. As mesmas referências sobre $\mathrm{o}$ ${ }^{92} \mathrm{Zr}$ afirmam que os seus estados $5^{-},\left(7^{-}\right)$e $\left(9^{-}\right)$, os quais são comparados à banda de paridade negativa teórica, também são populados na reação ${ }^{88} \mathrm{Sr}\left({ }^{7} \mathrm{Li}, 2 n p \gamma\right){ }^{92} \mathrm{Zr}$. A respeito da banda de estado fundamental do ${ }^{94} \mathrm{Mo}$, a pesquisa no seu espectro geral [40] e na Ref. [41] mostra que os níveis experimentais entre $0^{+}$e $6^{+}$são populados na reação ${ }^{90} \mathrm{Zr}\left({ }^{16} \mathrm{O},{ }^{12} \mathrm{C} \gamma\right){ }^{94} \mathrm{Mo}$, a qual configura uma forma de transferência- $\alpha$ no ${ }^{90} \mathrm{Zr}$. Tais dados experimentais podem ser interpretados como indicações da estrutura de cluster- $\alpha$ nos estados mencionados, favorecendo parcialmente as previsões do modelo de cluster- $\alpha$ para os núcleos ${ }^{92} \mathrm{Zr}$ e ${ }^{94} \mathrm{Mo}$.

4. O cálculo das variações relativas $\left(\Delta V_{0}\right)_{\text {rel }}$, as quais são necessárias para a reprodução correta dos níveis experimentais das bandas de estado fundamental, demonstra que o valor $V_{0}=220 \mathrm{MeV}$ é bastante apropriado para uma descrição geral dos espectros dos cinco núcleos da região do Mo. Adicionalmente, é mostrado que o grau de eficiência do conjunto fixo $\left(V_{0}, a, b\right)$ na região do Mo é próximo daquele observado nas bandas de estado fundamental dos núcleos ${ }^{20} \mathrm{Ne},{ }^{44} \mathrm{Ti}$ e ${ }^{212} \mathrm{Po}[3]$.

5. O reajuste do parâmetro $V_{0}$ para a reprodução correta dos níveis experimentais das bandas de estado fundamental revela que este parâmetro apresenta uma fraca dependência em relação ao número quântico $L$, sendo que tal dependência, de forma geral, é semelhante para os cinco núcleos da região do Mo. Os gráficos $V_{0} \times L$ referentes aos cinco núcleos são descritos simultaneamente e de forma satisfatória através de duas funções $V_{0}(L)$ (ver Subseção 4.1.2), as quais são definidas por apenas dois pontos $\left(L_{\min }, V_{0}^{\min }\right)$ e $\left(L_{\max }, V_{0}^{\max }\right)$ que caracterizam os cinco núcleos. Uma pequena variação em $V_{0}^{\max }$ permite que as funções $V_{0}(L)$ também descrevam de forma razoável os gráficos $V_{0} \times L$ dos núcleos ${ }^{20} \mathrm{Ne},{ }^{44} \mathrm{Ti},{ }^{94} \mathrm{Mo} \mathrm{e}{ }^{212} \mathrm{Po}$ simultaneamente. Demonstra-se, portanto, que as funções $V_{0}(L)$ servem como termos de correção (ou refinamento) do potencial nuclear W.S. + W.S. ${ }^{3}$ na região específica do Mo ou para a descrição geral das diferentes regiões de massa.

6. Verifica-se que os valores do parâmetro $R$ para os cinco núcleos da região do Mo apresentam um comportamento aproximadamente linear em relação a $A_{T}^{1 / 3}$ e $A_{\alpha}^{1 / 3}+A_{\text {car }}^{1 / 3}$, sendo melhor descritos pelas relações:

$$
R=4.723 A_{T}^{1 / 3}-15.867(\mathrm{fm}) \quad \text { e } \quad R=4.588\left(A_{\alpha}^{1 / 3}+A_{\text {car }}^{1 / 3}\right)-22.237(\mathrm{fm}) .
$$


Também verifica-se que os valores de $R$ para os núcleos ${ }^{20} \mathrm{Ne},{ }^{44} \mathrm{Ti},{ }^{94} \mathrm{Mo} \mathrm{e}$ ${ }^{212}$ Po comportam-se de forma tipicamente linear em relação a $A_{T}^{1 / 3}$ e $A_{\alpha}^{1 / 3}+A_{\text {car }}^{1 / 3}$, sendo que a melhor descrição para os 4 raios é dada por:

$$
R=1.092\left(A_{\alpha}^{1 / 3}+A_{\text {car }}^{1 / 3}\right)-1.041(\mathrm{fm}) .
$$

Porém, demonstra-se que a relação de proporcionalidade

$$
R=1.224 A_{T}^{1 / 3}(\mathrm{fm})
$$

é a mais apropriada para a descrição simultânea dos cinco núcleos da região do Mo e dos núcleos de diferentes regiões de massa.

7. O cálculo das separações intercluster rms indica a presença do efeito antistretching nas bandas de estado fundamental dos cinco núcleos da região do Mo. Este fato concorda com as previsões de Ohkubo [4] a respeito do ${ }^{94}$ Mo. O cálculo das razões $\left\langle r^{2}\right\rangle^{1 / 2} /\left(\left\langle R^{2}\right\rangle_{\alpha}^{1 / 2}+\left\langle R^{2}\right\rangle_{\text {car }}^{1 / 2}\right)$ para os cinco núcleos indica que há uma superposição entre o cluster- $\alpha$ e o caroço que já é significativa nos estados $0^{+}$e torna-se maior nos últimos níveis das bandas de estado fundamental, sugerindo que a estrutura de cluster- $\alpha$ é mais compacta nos níveis de maior spin das mesmas bandas. Revela-se que o número quântico $G$ exerce influência sobre as razões $\left\langle r^{2}\right\rangle^{1 / 2} /\left(\left\langle R^{2}\right\rangle_{\alpha}^{1 / 2}+\left\langle R^{2}\right\rangle_{\text {car }}^{1 / 2}\right)$ de tal maneira que os núcleos associados a $G_{\min }=14\left({ }^{90} \mathrm{Sr}\right.$ e $\left.{ }^{92} \mathrm{Zr}\right)$ formam um grupo com maior grau de compactação na estrutura $\alpha+$ caroço, enquanto que os núcleos associados a $G_{\min }=16\left({ }^{94} \mathrm{Mo},{ }^{96} \mathrm{Ru}\right.$ e $\left.{ }^{98} \mathrm{Pd}\right)$ formam outro grupo com menor grau de compactação. A análise individual dos cinco núcleos mostra que o ${ }^{94}$ Mo é aquele com o menor grau de compactação da estrutura $\alpha+$ caroço, corroborando as afirmações da literatura de que o ${ }^{94}$ Mo é o núcleo preferencial para a investigação da estrutura de cluster- $\alpha$ na sua região de massa.

8. O cálculo das larguras- $\alpha$ reduzidas $\gamma_{\alpha}^{2}$ e das larguras- $\alpha$ reduzidas adimensionais $\theta_{\alpha}^{2}$ para as bandas de estado fundamental dos cinco núcleos da região do Mo aponta que os estados de spins mais baixos estão mais fortemente associados à estrutura de cluster- $\alpha$ do que aqueles de maiores spins. Este resultado concorda com as previsões fornecidas pelas razões $\left\langle r^{2}\right\rangle^{1 / 2} /\left(\left\langle R^{2}\right\rangle_{\alpha}^{1 / 2}+\left\langle R^{2}\right\rangle_{\text {car }}^{1 / 2}\right)$ nas mesmas bandas. Por outro lado, mesmo os valores mais altos obtidos para $\gamma_{\alpha}^{2}$ representam frações muito pequenas do limite de Wigner, indicando que as bandas de estado fundamental dos cinco núcleos possuem, de forma geral, uma fraca característica de aglomeração- $\alpha$. Revela-se que o número quântico $G$ exerce influência sobre as larguras- $\alpha$ reduzidas de forma similar ao efeito observado nas razões $\left\langle r^{2}\right\rangle^{1 / 2} /\left(\left\langle R^{2}\right\rangle_{\alpha}^{1 / 2}+\left\langle R^{2}\right\rangle_{\text {car }}^{1 / 2}\right)$, pois os 
valores de $\theta_{\alpha}^{2}$ sugerem que os núcleos associados a $G_{\min }=14$ formam um grupo com intensidade de aglomeração- $\alpha$ mais baixa e os núcleos associados a $G_{\min }=16$ formam outro grupo com intensidade de aglomeração- $\alpha$ mais alta. O núcleo ${ }^{94} \mathrm{Mo}$ é aquele que apresenta os maiores valores de $\theta_{\alpha}^{2}$ entre os cinco núcleos analisados, o que corrobora, juntamente com os resultados das razões $\left\langle r^{2}\right\rangle^{1 / 2} /\left(\left\langle R^{2}\right\rangle_{\alpha}^{1 / 2}+\left\langle R^{2}\right\rangle_{\text {car }}^{1 / 2}\right)$, as afirmações da literatura de que o ${ }^{94}$ Mo é o núcleo mais favorável para a investigação da estrutura de cluster- $\alpha$ nesta região de massa.

9. As larguras- $\alpha$ reduzidas calculadas para as bandas de estado fundamental dos núcleos ${ }^{20} \mathrm{Ne},{ }^{44} \mathrm{Ti},{ }^{94} \mathrm{Mo} \mathrm{e}{ }^{212} \mathrm{Po}$ possuem diferentes ordens de grandeza, mostrando que os núcleos de menor massa apresentam as maiores larguras- $\alpha$ reduzidas, enquanto que os de maior massa apresentam as menores larguras$\alpha$ reduzidas. Desta forma, indica-se uma condição mais fraca de aglomeração$\alpha$ para os núcleos da região do Mo em relação aos núcleos leves. No entanto, mesmo os valores de $\gamma_{\alpha}^{2}$ para o ${ }^{20} \mathrm{Ne}$ representam pequenas frações do limite de Wigner, significando que as bandas de estado fundamental dos quatro núcleos possuem uma fraca característica de aglomeração- $\alpha$ em geral, sendo que esta condição se intensifica fortemente para os núcleos mais pesados.

10. As taxas de transição $B(E 2)$ calculadas para as bandas de estado fundamental dos núcleos ${ }^{90} \mathrm{Sr},{ }^{92} \mathrm{Zr},{ }^{94} \mathrm{Mo}$ e ${ }^{96} \mathrm{Ru}$ apresentam, com poucas exceções, a mesma ordem de grandeza dos valores experimentais, o que representa uma descrição satisfatória, pois não são usadas cargas efetivas nos cálculos e os parâmetros do potencial $\alpha+$ caroço não são ajustados com referência nos dados experimentais de $B(E 2)$. É constatado que a escolha dos números quânticos $G=14$ para ${ }^{90} \mathrm{Sr}$ e ${ }^{92} \mathrm{Zr}$ e $G=16$ para ${ }^{94} \mathrm{Mo} \mathrm{e}{ }^{96} \mathrm{Ru}$ é favorável para uma melhor descrição das taxas de transição experimentais. O modelo de cluster- $\alpha$ demonstra ser mais eficiente na reprodução dos dados experimentais de $B(E 2)$ em comparação com cálculos baseados no modelo de camadas, pois altas cargas efetivas são necessárias para que o modelo de camadas consiga reproduzir as ordens de grandeza dos mesmos dados experimentais [53]. Assim, os resultados sugerem que a consideração da aglomeração- $\alpha$ nos núcleos da região do Mo contribui para uma reprodução mais coerente das taxas de transição.

11. É mostrado que o potencial $\alpha+$ caroço na forma W.S. + W.S. ${ }^{3}$ fornece uma reprodução geral satisfatória dos espaçamentos entre os níveis experimentais das bandas de paridade negativa dos núcleos ${ }^{92} \mathrm{Zr},{ }^{94} \mathrm{Mo},{ }^{96} \mathrm{Ru}$ e ${ }^{98} \mathrm{Pd}$, considerando que os parâmetros $a, b$ e $R$ foram mantidos com os mesmos valores aplicados na descrição das bandas de estado fundamental e que o parâmetro 
$V_{0}$ necessita de uma variação baixa em relação ao valor $V_{0}=220 \mathrm{MeV}$ (entre $7.7 \%$ e $8.6 \%$ ) para uma descrição mais adequada das bandas de paridade negativa. Infelizmente, as quatro bandas experimentais são incompletas e a maioria dos níveis conhecidos não possuem spin e/ou paridade confirmados, impedindo um julgamento mais consistente das bandas calculadas. Sugerese que o potencial $\alpha$ + caroço possui uma fraca dependência da paridade, contudo, não desprezível.

12. Mostra-se uma tendência de padronização do parâmetro $V_{0}$ para a descrição das bandas de paridade negativa dos núcleos ${ }^{92} \mathrm{Zr}$, ${ }^{94} \mathrm{Mo},{ }^{96} \mathrm{Ru}$ e ${ }^{98} \mathrm{Pd}$. Conforme os cálculos realizados, indica-se que $V_{0}=237 \mathrm{MeV}$ é um valor adequado para a descrição geral das bandas associadas a $G=17$; entretanto, a profundidade mais conveniente para a descrição geral dos quatro núcleos deve ser $V_{0} \approx 237.5 \mathrm{MeV}$.

13. A análise dos níveis teóricos de maior spin das bandas de paridade negativa dos núcleos ${ }^{92} \mathrm{Zr},{ }^{94} \mathrm{Mo},{ }^{96} \mathrm{Ru}$ e ${ }^{98} \mathrm{Pd}$ revela que os estados de tais bandas acima do limiar $\alpha+$ caroço são praticamente ligados.

Os resultados mencionados levam à seguinte conclusão geral:

A aplicação do modelo de cluster- $\alpha$ com Abordagem de Potencial Local nos núcleos da região do Molibdênio demonstra que este modelo pode ser estendido, com resultados favoráveis, para outros núcleos além daqueles de configuração $\alpha+$ (caroço de dupla camada fechada). Adicionalmente, indica-se que os núcleos de $A$ par e $N=52$ na região do Molibdênio possuem estruturas $\alpha+$ caroço com características semelhantes.

São perspectivas decorrentes deste trabalho a inclusão de outros núcleos da mesma região. A extensão dos cálculos para núcleos ímpares também é possível; entretanto, para tais núcleos, são necessárias adaptações como a inclusão de termos não-centrais na Hamiltoniana do sistema. Novos dados experimentais serão de grande importância para o aperfeiçoamento deste trabalho, particularmente os dados referentes às reações de transferência- $\alpha$.

Outras subregiões de massa ainda não foram exploradas sistematicamente através do modelo de cluster- $\alpha$, significando que muitos núcleos ainda podem ser analisados pelos métodos apresentados neste trabalho. Linhas de pesquisa alternativas podem ser desenvolvidas com os mesmos princípios, como a procura por outras estruturas envolvendo aglomerados maiores que $\alpha$ [57] ou estados do tipo molecular em núcleos leves [58]. Assim, este trabalho serve como uma referência para estudos futuros nesta área. 


\section{Apêndice A}

\section{Determinação das funções $V_{0}(L)$}

Neste apêndice, mostra-se como foram determinadas as funções $V_{0}(L)$ usadas para a descrição do comportamento do parâmetro $V_{0}$ em relação ao número quântico $L$.

\section{A.1 Adaptação da função $y_{1}(x)=a_{1} x^{3}+b_{1} x^{2}$ para a descrição do parâmetro $V_{0}$}

A adaptação das funções $y_{1}(x)$ e $y_{2}(x)$ em (4.2) e (4.3) para a descrição dos gráficos $V_{0} \times L$ depende da definição do ponto de mínimo local $\left(L_{\min }, V_{0}^{\min }\right)$ e do ponto de máximo local $\left(L_{\max }, V_{0}^{\max }\right)$ das funções resultantes $V_{0}(L)$. Como tais pontos já são definidos nas Subseções 4.1 .2 e 4.1.3, necessita-se então relacionar $\left(L_{\min }, V_{0}^{\min }\right)$ e $\left(L_{\max }, V_{0}^{\max }\right)$ com os pontos de máximo e mínimo das funções $y_{1}(x)$ e $y_{2}(x)$. Tratando inicialmente da função

$$
y_{1}(x)=a_{1} x^{3}+b_{1} x^{2},
$$

deve-se resolver a equação

$$
\frac{d y_{1}}{d x}=0
$$

para o cálculo dos pontos de derivada nula em $y_{1}(x)$. As soluções da eq. (A.2) são:

$$
x_{\min }=0 \quad \text { e } \quad x_{\max }=-\frac{2 b_{1}}{3 a_{1}} .
$$

É estabelecido que as soluções $x_{\min }$ e $x_{\max }$ estão associadas aos pontos de mínimo local e máximo local de $y_{1}(x)$, respectivamente.

Dando início à mudança das coordenadas $x$ e $y_{1}$ para $L$ e $V_{0}$, será feita uma translação da função $y_{1}(x)$ de tal forma que o ponto de mínimo $\left(x_{\min }, y_{\min }\right)=(0,0)$ 
seja vinculado a $\left(L_{\min }, V_{0}^{\text {min }}\right)$. As equações que definem a mudança de coordenadas são:

$$
x=L-L_{\min }
$$

e

$$
y_{1}=V_{0}-V_{0}^{\min }
$$

Aplicando (A.4) e (A.5) em (A.1), temos

$$
V_{0}(L)=a_{1}\left(L-L_{\min }\right)^{3}+b_{1}\left(L-L_{\min }\right)^{2}+V_{0}^{\min },
$$

onde $V_{0}(L)$ é a função polinomial adaptada para a descrição do parâmetro $V_{0}$.

A determinação das constantes $a_{1}$ e $b_{1}$ ainda é necessária. Para isto, será estabelecido o vínculo entre o ponto de máximo $\left(x_{\max }, y_{\max }\right)$ do antigo sistema de coordenadas e $\left(L_{\max }, V_{0}^{\max }\right)$. Usando a eq. (A.4), temos que

$$
L_{\max }-L_{\min }=x_{\max }=-\frac{2 b_{1}}{3 a_{1}}
$$

o que resulta em

$$
b_{1}=-\frac{3 a_{1}}{2}\left(L_{\max }-L_{\min }\right) \text {. }
$$

Aplicando (A.8) em (A.6) e usando o fato de que $V_{0}\left(L_{\max }\right)=V_{0}^{\max }$, obtém-se a constante $a_{1}$, dada por:

$$
a_{1}=-\frac{2\left(V_{0}^{\max }-V_{0}^{\min }\right)}{\left(L_{\max }-L_{\min }\right)^{3}} .
$$

Por fim, aplicando (A.9) em (A.8), obtém-se a constante $b_{1}$, dada por:

$$
b_{1}=\frac{3\left(V_{0}^{\max }-V_{0}^{\min }\right)}{\left(L_{\max }-L_{\min }\right)^{2}} .
$$

\section{A.2 Adaptação da função $y(x)=\exp \left(a_{2} x\right) / \exp \left(b_{2} x^{3}\right)$ para a descrição do parâmetro $V_{0}$}

Repetindo o procedimento da seção anterior, deve-se calcular os pontos de derivada nula da função

$$
y_{2}(x)=\frac{\exp \left(a_{2} x\right)}{\exp \left(b_{2} x^{3}\right)}
$$

pela resolução da equação 


$$
\frac{d y_{2}}{d x}=0
$$

As soluções para a eq. (A.12) são:

$$
x_{\min }=-\alpha \quad \text { e } \quad x_{\max }=\alpha, \quad \text { onde } \quad \alpha=\sqrt{\frac{a_{2}}{3 b_{2}}} .
$$

É estabelecido que as soluções $x_{\min }$ e $x_{\max }$ estão associadas aos pontos de mínimo local e máximo local de $y_{2}(x)$, respectivamente.

Para o caso da função (A.11), não é possível a mudança das coordenadas $x$ e $y_{2}$ diretamente para $L$ e $V_{0}$, pois não há um coeficiente externo à expressão $\exp \left(a_{2} x\right) / \exp \left(b_{2} x^{3}\right)$ para que a dimensão de energia seja atribuída à função resultante $V_{0}(L)$. Assim, a mudança das coordenadas $x$ e $y_{2}$ é feita primeiramente para $L$ e $\lambda$, onde $\lambda$ é uma variável adimensional relacionada a $V_{0}$ por

$$
\lambda=\frac{V_{0}}{V_{0}^{\min }} .
$$

A função adimensional resultante $\lambda(L)$ possui os pontos de mínimo e máximo local $\left(L_{\min }, \lambda_{\min }\right)$ e $\left(L_{\max }, \lambda_{\max }\right)$, respectivamente, onde $\lambda_{\min }$ e $\lambda_{\max }$ estão associados respectivamente a $V_{0}^{\min }$ e $V_{0}^{\max }$ através da eq. (A.14).

Para a mudança das coordenadas $x$ e $y_{2}$ para $L$ e $\lambda$, será feita uma translação da função $y_{2}(x)$ de tal forma que o ponto de mínimo

$$
\left(x_{\min }, y_{\min }\right)=\left(-\alpha, \frac{\exp \left(-a_{2} \alpha\right)}{\exp \left(-b_{2} \alpha^{3}\right)}\right)
$$

seja vinculado a $\left(L_{\min }, \lambda_{\min }\right)$. As equações que definem a mudança de coordenadas são:

$$
x=L-L_{\min }-\alpha
$$

e

$$
y_{2}=\lambda-\lambda_{\min }+\frac{\exp \left(-a_{2} \alpha\right)}{\exp \left(-b_{2} \alpha^{3}\right)} .
$$

Aplicando (A.16) e (A.17) em (A.11), temos

$$
\lambda(L)=\frac{\exp \left[a_{2}\left(L-L_{\min }-\alpha\right)\right]}{\exp \left[b_{2}\left(L-L_{\min }-\alpha\right)^{3}\right]}+\lambda_{\min }-\frac{\exp \left(-a_{2} \alpha\right)}{\exp \left(-b_{2} \alpha^{3}\right)},
$$

onde $\lambda(L)$ é a função do tipo $\exp \left(a_{2} x\right) / \exp \left(b_{2} x^{3}\right)$ adaptada para a descrição da variável adimencional $\lambda$. 
A determinação das constantes $a_{2}$ e $b_{2}$ ainda é necessária. Para isto, será estabelecido o vínculo entre o ponto de máximo $\left(x_{\max }, y_{\max }\right)$ do antigo sistema de coordenadas e $\left(L_{\max }, \lambda_{\max }\right)$. Usando a eq. (A.16), temos que

$$
L_{\max }-L_{\min }=x_{\max }+\alpha=2 \sqrt{\frac{a_{2}}{3 b_{2}}},
$$

o que resulta em

$$
a_{2}=\frac{3 b_{2}}{4}\left(L_{\max }-L_{\min }\right)^{2}
$$

e

$$
\alpha=\frac{1}{2}\left(L_{\max }-L_{\min }\right)
$$

Aplicando (A.20) e (A.21) em (A.18) e usando o fato de que $\lambda\left(L_{\max }\right)=\lambda_{\max }$, obtém-se a equação:

$$
\begin{aligned}
& \lambda_{\max }= \\
& \quad \frac{\exp \left[\left(3 b_{2} / 8\right)\left(L_{\max }-L_{\min }\right)^{3}\right]}{\exp \left[\left(b_{2} / 8\right)\left(L_{\max }-L_{\min }\right)^{3}\right]}+\lambda_{\min }-\frac{\exp \left[-\left(3 b_{2} / 8\right)\left(L_{\max }-L_{\min }\right)^{3}\right]}{\exp \left[-\left(b_{2} / 8\right)\left(L_{\max }-L_{\min }\right)^{3}\right]}
\end{aligned}
$$

Desenvolvendo os termos da eq. (A.22), obtém-se a constante $b_{2}$, dada por

$$
b_{2}=\frac{4}{\left(L_{\max }-L_{\min }\right)^{3}} \operatorname{arcsenh}\left(\frac{\lambda_{\max }-\lambda_{\min }}{2}\right),
$$

e aplicando (A.23) em (A.20), obtém-se a constante $a_{2}$, dada por

$$
a_{2}=\frac{3}{L_{\max }-L_{\min }} \operatorname{arcsenh}\left(\frac{\lambda_{\max }-\lambda_{\min }}{2}\right) .
$$

Por fim, a obtenção da função $\lambda(L)$ e das constantes $a_{2}$ e $b_{2}$ permite que a função $V_{0}(L)$ seja determinada pela relação

$$
V_{0}(L)=V_{0}^{\min } \lambda(L),
$$

a qual é conseqüência da eq. (A.14). 


\section{Apêndice B}

\section{Estrutura de cluster- $\alpha$ no ${ }^{13} \mathbf{C}$}

Neste apêndice, discute-se a estrutura de cluster- $\alpha$ no ${ }^{13} \mathrm{C}$ através de dois estudos realizados paralelamente ao trabalho sobre os núcleos da região do Mo. Os resultados dos referidos estudos são apresentados em dois artigos reproduzidos dentro deste apêndice. A seguir, são feitas breves descrições de cada artigo.

\section{B.1 Descrição do artigo "Simple description of alpha-cluster states in ${ }^{13} \mathrm{C} "$}

Este artigo [59] foi publicado nos Proceedings of the XXX Reunião de Trabalho sobre Física Nuclear no Brasil. Vários níveis experimentais de paridades positiva e negativa do ${ }^{13} \mathrm{C}$ são interpretados em termos de um sistema $\alpha+{ }^{9} \mathrm{Be}$, onde considerase o ${ }^{9} \mathrm{Be}$ como um caroço no seu estado fundamental $\left(J^{\pi}=3 / 2^{-}\right)$. O cálculo serve como um exemplo do uso do modelo de cluster- $\alpha$ com Abordagem de Potencial Local em sistemas com a presença de forças não-centrais na interação $\alpha+$ caroço.

\section{B.2 Descrição do artigo "Investigation of $\alpha$-cluster states in ${ }^{13} \mathrm{C}$ via the $\left({ }^{6} \mathrm{Li}, \mathrm{d}\right)$ reaction"}

Este artigo [60] foi publicado nos Proceedings of the $12^{\text {th }}$ International Conference on Nuclear Reaction Mechanisms (Varenna, Itália), e mostra dados experimentais recentes sobre a reação ${ }^{9} \mathrm{Be}\left({ }^{6} \mathrm{Li}, d\right){ }^{13} \mathrm{C}$. Entre outras informações, o referido trabalho mostra a aplicação das funções radiais $u_{G L J}(r)$, as quais estão associadas aos estados $|G, L, J\rangle$ do sistema $\alpha+{ }^{9} \mathrm{Be}$, na análise de DWBA de duas distribuições angulares referentes às seções de choque de transferência- $\alpha$ no ${ }^{9} \mathrm{Be}$. 
Informações sobre a origem das funções radiais encontram-se no artigo citado na Seção B.1. 


\title{
Simple description of alpha-cluster states in ${ }^{13} \mathrm{C}$
}

\author{
M. A. Souza* and H. Miyake ${ }^{\dagger}$ \\ Instituto de Física, Universidade de São Paulo, São Paulo, Brazil
}

\begin{abstract}
We use a simple form of the $\alpha$-cluster model to describe the energy levels of ${ }^{13} \mathrm{C}$. These states are interpreted in terms of an $\alpha+{ }^{9} \mathrm{Be}$ system, where the ${ }^{9} \mathrm{Be}$ nucleus is considered as an inert core in its ground state. A local cluster-core potential is adopted for the calculation of bound and resonant states. We present the energy levels obtained for the positive and negative parity bands and the respective $\alpha$ reduced widths in comparison with experimental levels of ${ }^{13} \mathrm{C}$.
\end{abstract}

\section{INTRODUCTION}

The alpha-cluster model has already been applied in the light mass region in several works $[1,3,14]$, producing a good description of spectroscopic properties in this region. More sophisticated models involving molecular structures such as $i \alpha$ or $i \alpha+$ nucleon $(i=1,2,3, \ldots)$ have been employed in light nuclei with satisfactory results $[4,14]$. However, the simplest form of the $\alpha$-cluster model, that is, the model where the nuclear states are described through an $\alpha+$ core system, can provide more easily information corcerning energy levels, electromagnetic transition rates, $\alpha$-emission widths and reduced $\alpha$-widths for a significant group of states, also generating simple radial wave functions that may be used in DWBA calculations for the study of $\alpha$-transfer reactions. A motivation for new applications of this model in light nuclei is the renewed interest in the research on the $\alpha$-cluster structure, particularly by means of the $\left({ }^{6} \mathrm{Li}, \mathrm{d}\right)$ transfer reaction in odd $A$ light nuclei [2]. Thus, we have studied the ${ }^{13} \mathrm{C}$ nucleus by treating its states in terms of an $\alpha+{ }^{9}$ Be system, where internal excitation effects of the constituents are not considered. The $\alpha-{ }^{9} \mathrm{Be}$ interaction is produced by a local phenomenological potential. We present the calculated spectrum for the negative and positive parity bands and the $\alpha$ reduced widths obtained for the $\alpha+{ }^{9} \mathrm{Be}$ states.

\section{DESCRIPTION OF THE $\alpha+{ }^{9}$ Be SYSTEM}

A local cluster-core potential is used to calculate the states of the $\alpha-{ }^{9} \mathrm{Be}$ system. The complete potential is given by

$$
V(r)=-V_{0} f(r)+V_{S O}(r)+V_{C}(r)
$$

where $-V_{0} f(r), V_{S O}(r)$ and $V_{C}(r)$ are the nuclear, spinorbit and Coulomb terms, respectively. For the nuclear potential, we adopt the form

\footnotetext{
*Electronic address: marsouza@if.usp.br

${ }^{\dagger}$ Electronic address: miyake@if.usp.br
}

$$
f(r)=\frac{b}{1+\exp \left(\frac{r-R}{a}\right)}+\frac{1-b}{\left[1+\exp \left(\frac{r-R}{3 a}\right)\right]^{3}}
$$

proposed by Buck, Merchant and Perez [5]. The Coulomb potential $V_{C}(r)$ is taken to be that of an uniform spherical charge distribution of radius $R_{C}=R$. The form of the spin-orbit potential is

$$
V_{S O}(r)=V_{S O}\left(\frac{\hbar}{m_{\pi} c}\right)^{2} \frac{1}{r} \frac{d f(r)}{d r} \mathbf{I} \cdot \mathbf{L},
$$

where $\left(\hbar / m_{\pi} c\right)^{2}=2 \mathrm{fm}^{2}$ and $\mathbf{I}$ is the spin of the ${ }^{9}$ Be core in its ground state $\left(I^{\pi}=3 / 2^{-}\right)$.

The $\alpha-{ }^{9} \mathrm{Be}$ states are identified by the quantum numbers $G, L$ and $J$, where $G$ identifies the band of states, $L$ is the orbital angular momentum and $J$ is the total angular momentum. The values of the total angular momentum $J$ are obtained through the coupling of $L$ with the spin of the ${ }^{9} \mathrm{Be}$ inert core $\left(I^{\pi}=3 / 2^{-}\right)$. The $J^{\pi}$ states generated by the model with the spin-orbit interaction are presented in Table I.

We adopt $G=4$ as the quantum number of the lowest band of the $\alpha+{ }^{9} \mathrm{Be}$ system. This value is obtained from the Wildermuth condition [6] considering the four nucleons of the $\alpha$-cluster in the $p$ shell.

The parameters of $V(r)$ have been fitted to give a satisfactory reproduction of the ${ }^{13} \mathrm{C}$ experimental levels populated in $\alpha$-transfer reactions, taking into account the $\alpha$-separation energy. In this way, the depth parameter $V_{0}$ has been fitted separately for the $G=4$ and $G=5$ bands. The values employed for the nuclear potential are: $V_{0}=103.9 \mathrm{MeV}$ for the $G=4$ band, $V_{0}=115.5 \mathrm{MeV}$ for the $G=5$ band, $R=3.22 \mathrm{fm}$ and $a=0.65 \mathrm{fm}$. We also adopt $b=0.3$ since this value is appropriate for the description of the $\alpha$-cluster structure in several nuclei of different mass regions [5].

The spin-orbit parameter $V_{S O}$ has been fitted separately for each set of states produced by the splitting of a $|G, L\rangle$ state of the $\alpha+{ }^{9} \mathrm{Be}$ system with no spinorbit interaction (see Figs. 1 and 2). The values employed for the spin-orbit parameter are: $V_{S O}=8.95 \mathrm{MeV}$ for $|G=4, L=2\rangle, V_{S O}=3.48 \mathrm{MeV}$ for $|G=4, L=4\rangle$, $V_{S O}=10.90 \mathrm{MeV}$ for $|G=5, L=1\rangle$ and $V_{S O}=5.98$ $\mathrm{MeV}$ for $|G=5, L=3\rangle$. We have not calculated the spin-orbit parameter for the $|G=5, L=5\rangle$ state, since 
TABLE I: Theoretical states of the $\alpha-{ }^{9}$ Be system. The corresponding quantum numbers $L$ and $G$ are indicated.

\begin{tabular}{ccc}
\hline \hline$G$ & $L$ & $J^{\pi}$ \\
\hline 4 & 0 & $\frac{3}{2}^{-}$ \\
4 & 2 & $\frac{1}{2}^{-}, \frac{3}{2}^{-}, \frac{5}{2}^{-}, \frac{7}{2}^{-}$ \\
4 & 4 & $\frac{5}{2}^{-}, \frac{7}{2}^{-}, \frac{9}{2}^{-}, \frac{11}{2}^{-}$ \\
5 & 1 & $\frac{1}{2}^{+}, \frac{3}{2}^{+}, \frac{5}{2}^{+}$ \\
5 & 3 & $\frac{3}{2}^{+}, \frac{5}{2}^{+}, \frac{7}{2}^{+}, \frac{9}{2}^{+}$ \\
5 & 5 & $\frac{7}{2}^{+}, \frac{9}{2}^{+}, \frac{11}{2}^{+}, \frac{13}{2}^{+}$ \\
\hline \hline
\end{tabular}

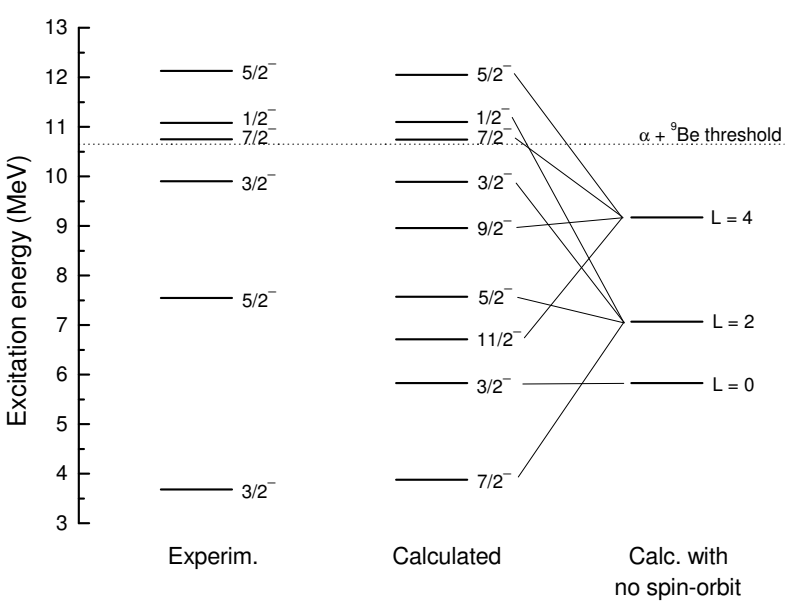

FIG. 1: Comparison of the calculated negative parity states of the $\alpha+{ }^{9}$ Be system $(G=4$ band) with experimental excitation energies of ${ }^{13} \mathrm{C}$. The experimental values are from Ref. [7].

the spin-orbit splitting in this case produces higher spin states (see Table I) that have not been detected experimentally or lie in energies above $E_{x} \approx 13 \mathrm{MeV}$, where the spins and parities of many ${ }^{13} \mathrm{C}$ states are uncertain.

\section{RESULTS AND CONCLUSION}

A comparison of the calculated $\alpha-{ }^{9}$ Be states with experimental energy levels of ${ }^{13} \mathrm{C}[7]$ is shown in Figs. 1 and 2. Most of the compared experimental energy levels are populated in $\alpha$-transfer reactions as ${ }^{9} \mathrm{Be}\left({ }^{6} \mathrm{Li}, \mathrm{d}\right){ }^{13} \mathrm{C}[7,8]$ and ${ }^{9} \mathrm{Be}\left({ }^{7} \mathrm{Li}, \mathrm{t}\right){ }^{13} \mathrm{C}[7,9]$ and several levels shown above the $\alpha+{ }^{9} \mathrm{Be}$ threshold are detected in different $\alpha$-decay processes of ${ }^{13} \mathrm{C}[7,11,12]$ and the $\alpha$ elastic scattering on ${ }^{9} \mathrm{Be}[7,10]$.

We have also calculated the radial wave functions associated with the $\alpha-{ }^{9} \mathrm{Be}$ states. For the theoretical states with corresponding experimental energy levels, we have determined the wave functions by adjusting separately the depth parameter $V_{0}$ for each state in order to obtain the correct energies (see Table II). For the theoreti-

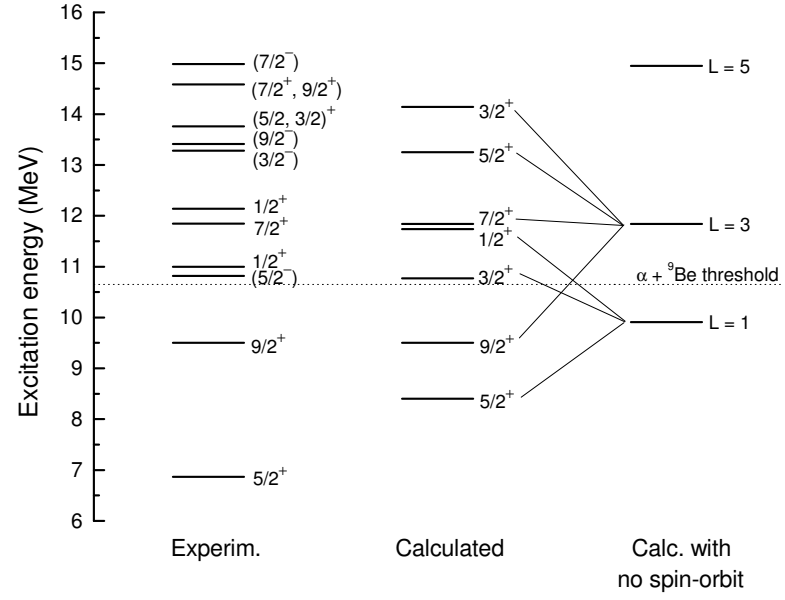

FIG. 2: Comparison of the calculated positive parity states of the $\alpha+{ }^{9}$ Be system $(G=5$ band) with experimental excitation energies of ${ }^{13} \mathrm{C}$. The experimental values are from Ref. [7].

cal states with no corresponding experimental levels, we have applied the same depth values mentioned in Section II.

The radial wave functions have been used for the calculation of the $\alpha$ reduced widths of the $\alpha{ }^{-9}$ Be states. According to the $R$-matrix theory [13], the $\alpha$ reduced width $\gamma_{\alpha ; G L J}^{2}$ of a $|G, L, J\rangle$ state is given by

$$
\gamma_{\alpha ; G L J}^{2}=\left(\frac{\hbar^{2}}{2 \mu a}\right) u_{G L J}^{2}(a)\left[\int_{0}^{a}\left|u_{G L J}(r)\right|^{2} d r\right]^{-1}
$$

where $u_{G L J}(r)$ is the radial wave function, $a$ is the channel radius and $\mu$ is the reduced mass of the system. The dimensionless reduced width $\theta_{\alpha ; G L J}^{2}$ is defined as the ratio of $\gamma_{\alpha ; G L J}^{2}$ to the Wigner limit, that is:

$$
\theta_{\alpha ; G L J}^{2}=\frac{2 \mu a^{2}}{3 \hbar^{2}} \gamma_{\alpha ; G L J}^{2}
$$

These expressions have been employed equally for resonant and bound states. A channel radius $a=5.1 \mathrm{fm}$ has been adopted, taking as reference the channel radius already used for the analysis of the $\alpha$-cluster structure in ${ }^{17} \mathrm{O}$ (5.5 fm - see Ref. [14]) and assuming that $a$ is proportional to $\left(A_{\alpha}^{1 / 3}+A_{\text {core }}^{1 / 3}\right)$. The values obtained for the $\alpha$ reduced widths are presented in Tables II and III.

In principle, an $\alpha$ reduced width close to the Wigner limit $\left(\theta_{\alpha}^{2} \approx 1\right)$ indicates that the corresponding state has a strong $\alpha$-cluster character. The $1 / 2^{+}(10.996 \mathrm{MeV})$, $1 / 2^{-}(11.080 \mathrm{MeV})$, and $1 / 2^{+}(12.14 \mathrm{MeV})$ energy levels are not identified as states populated in $\alpha$-transfer reactions; however, these states have significant $\theta_{\alpha}^{2}$ values (see Table II), suggesting that they present an important degree of $\alpha$-clustering. It is possible that these states are populated in $\alpha$-transfer reactions, but their identification 
TABLE II: Calculated $\alpha$ reduced widths for the ${ }^{13} \mathrm{C}$ states associated with theoretical states of the $\alpha{ }^{-}{ }^{9} \mathrm{Be}$ system. The columns show the experimental excitation energies $E_{x}$, the corresponding spins and parities $\left(J^{\pi}\right)$, the $L$ values attributed to the states, the depths $V_{0}$ used to fit each state at its experimental energy, the $\alpha$ reduced widths $\gamma_{\alpha}^{2}$ and the dimensionless $\alpha$ reduced widths $\theta_{\alpha}^{2}$. A channel radius $a=5.1 \mathrm{fm}$ has been used. The experimental energies are from Ref. [7].

\begin{tabular}{cccccc}
\hline \hline$E_{x}(\mathrm{MeV})$ & $J^{\pi}$ & $L$ & $V_{0}(\mathrm{MeV})$ & $\gamma_{\alpha}^{2}(\mathrm{keV})$ & $\theta_{\alpha}^{2}(\%)$ \\
\hline 3.685 & $3 / 2^{-}$ & 0 & 111.7 & 85 & 9.8 \\
6.864 & $5 / 2^{+}$ & 1 & 122.4 & 208 & 24.0 \\
7.547 & $5 / 2^{-}$ & 2 & 104.0 & 117 & 13.4 \\
9.500 & $9 / 2^{+}$ & 3 & 115.5 & 181 & 20.8 \\
9.897 & $3 / 2^{-}$ & 2 & 103.9 & 201 & 23.1 \\
10.753 & $7 / 2^{-}$ & 4 & 103.8 & 60 & 6.9 \\
10.996 & $1 / 2^{+a}$ & 1 & 121.5 & 541 & 62.2 \\
11.080 & $1 / 2^{-}$ & 2 & 104.0 & 281 & 32.3 \\
11.848 & $7 / 2^{+}$ & 3 & 115.4 & 315 & 36.2 \\
12.13 & $5 / 2^{-}$ & 4 & 103.6 & 85 & 9.7 \\
12.14 & $1 / 2^{+a}$ & 1 & 111.7 & 832 & 95.7 \\
\hline \hline
\end{tabular}

${ }^{a}$ The two indicated ${ }^{13} \mathrm{C}$ states are associated with the $|G=5, L=1, J=1 / 2\rangle \alpha$-cluster state.

TABLE III: Calculated $\alpha$ reduced widths for the $\alpha-{ }^{9}$ Be states without corresponding experimental ${ }^{13} \mathrm{C}$ levels. The columns show the calculated excitation energies $\left(E_{x}\right.$ calc.), the corresponding spins and parities $\left(J^{\pi}\right)$, the $L$ values attributed to the states, the $\alpha$ reduced widths $\gamma_{\alpha}^{2}$ and the dimensionless $\alpha$ reduced widths $\theta_{\alpha}^{2}$. A channel radius $a=5.1 \mathrm{fm}$ has been used.

\begin{tabular}{ccccc}
\hline \hline$E_{x}$ calc. $(\mathrm{MeV})$ & $J^{\pi}$ & $L$ & $\gamma_{\alpha}^{2}(\mathrm{keV})$ & $\theta_{\alpha}^{2}(\%)$ \\
\hline 3.88 & $7 / 2^{-}$ & 2 & 57 & 6.6 \\
6.71 & $11 / 2^{-}$ & 4 & 27 & 3.1 \\
8.96 & $9 / 2^{-}$ & 4 & 41 & 4.7 \\
10.77 & $3 / 2^{+}$ & 1 & 506 & 58.1 \\
13.25 & $5 / 2^{+}$ & 3 & 494 & 56.9 \\
14.14 & $3 / 2^{+}$ & 3 & 639 & 73.4 \\
\hline \hline
\end{tabular}

is difficult since they are very close to neighbouring levels which are strongly populated in the same reactions (see Ref. [8]).

Fig. 2 shows experimental levels with uncertain assignments above $E_{x}=13 \mathrm{MeV}$ which may be associated with the $5 / 2^{+}(G=5, L=3)$ and $3 / 2^{+}(G=5$, $L=3$ ) calculated states. We have determined the phase shift functions $\delta\left(E_{\text {c.m. }}\right)$ for the two theoretical states and then these functions have been used to calculate the total $\alpha$-widths $\Gamma_{\alpha}$ through the relation $\Gamma=2 /\left.(d \delta / d E)\right|_{E=E_{r}}$, where $E_{r}$ is the resonance energy. The obtained values are $\Gamma_{\alpha} \approx 0.21 \mathrm{MeV}$ for the $5 / 2^{+}(G=5, L=3)$ state and $\Gamma_{\alpha} \approx 0.83 \mathrm{MeV}$ for the $3 / 2^{+}(G=5, L=3)$ state. These values present the same order of magnitude of the $\Gamma_{\text {c.m. }}$ widths reported for the experimental states shown in Fig. 2 above $E_{x}=13 \mathrm{MeV}$ [7].

Comparing the $L$ values shown in Table II with the $L$ transfer values obtained through the DWBA analysis of the ${ }^{9} \mathrm{Be}\left({ }^{6} \mathrm{Li}, \mathrm{d}\right){ }^{13} \mathrm{C}$ reaction [8], it is observed that the $3 / 2^{-}(3.685 \mathrm{MeV}), 5 / 2^{+}(6.864 \mathrm{MeV}), 9 / 2^{+}(9.500 \mathrm{MeV})$ and $3 / 2^{-}(9.897 \mathrm{MeV})$ states have the same $L$ values for both cases. Such correspondence motivates the use of the $\alpha-{ }^{9}$ Be potential and the radial wave functions of this work in further analyses of $\alpha$-transfer reactions.

In conclusion, the presented results indicate that the $\alpha+{ }^{9} \mathrm{Be}$ model yields a good general description of several ${ }^{13} \mathrm{C}$ energy levels detected through $\alpha$-transfer reactions, $\alpha$-decay processes and $\alpha$ elastic scattering, suggesting a significant degree of $\alpha$-clustering for many ${ }^{13} \mathrm{C}$ states. New information concerning the mentioned reactions may contribute for a better analysis of our calculations.

\section{Acknowledgments}

The authors thank the members of the Nuclear Spectroscopy with Light Ions Group of IFUSP for the useful discussions. Using resources of the LCCA - Laboratory of Advanced Scientific Computation of the University of São Paulo.

This work was supported by CAPES.
[1] B. Buck, C.B. Dover and J.P. Vary, Phys. Rev. C 11, 1803 (1975).

[2] M.R.D. Rodrigues et. al., XXX Reunião de Trabalho sobre Física Nuclear no Brasil, Águas de Lindóia - SP, Brasil - Program and Abstracts, p. 36 (2007).

[3] B. Buck and A. A. Pilt, Nucl. Phys. A280, 133 (1977).

[4] M. Milin and W. von Oertzen, Eur. Phys. J. A 14, 295 (2002).

[5] B. Buck, A.C. Merchant and S.M. Perez, Phys. Rev. C 51, 559 (1995).

[6] K. Wildermuth and Y.C. Tang, A Unified Theory of the Nucleus (Academic Press, New York, 1977).
[7] F. Ajzenberg-Selove, Nucl. Phys. A523, 1 (1991).

[8] X. Aslanoglow et. al., Phys. Rev. C 40, 73 (1989).

[9] F.D. Snyder and M.A. Wagoner, Phys. Rev. 186, 999 (1969).

[10] J.D. Goss et. al., Phys. Rev. C 7, 1837 (1973).

[11] N. Soić et. al., Nucl. Phys. A728, 12 (2003).

[12] D.L. Price et. al., Nucl. Phys. A765, 263 (2006).

[13] A. Arima and S. Yoshida, Nucl. Phys. A219, 475 (1974).

[14] H. Furutani et al., Suppl. Prog. Theor. Phys. 68, 193 (1980). 


\title{
Investigation of $\alpha$-cluster states in ${ }^{13} \mathrm{C}$ via the $\left({ }^{6} \mathrm{Li}, \mathrm{d}\right)$ reaction
}

\author{
M.R.D. Rodrigues ${ }^{1,2}$, T. Borello-Lewin ${ }^{1}$, L.B. Horodynski-Matsushigue ${ }^{1}$, A. Cunsolo $^{2}$, \\ F. Cappuzzello', J.L.M. Duarte ${ }^{1}$, C.L. Rodrigues ${ }^{1}$, G.M. Ukita ${ }^{1,3}$, M.A. Souza ${ }^{1}$ and H. Miyake ${ }^{1}$ \\ ${ }^{1}$ Institute of Physics, Universidade de São Paulo, São Paulo - SP, Brazil \\ ${ }^{2}$ Departament of Physics and Astronomy, Università di Catania and I.N.F.N., \\ Laboratori Nazionali del Sud, Catania, Italy \\ ${ }^{3}$ Faculty of Psychology, Universidade de Santo Amaro, São Paulo - SP, Brazil
}

\begin{abstract}
The ${ }^{9} \mathrm{Be}\left({ }^{6} \mathrm{Li}, \mathrm{d}\right){ }^{13} \mathrm{C}$ reaction was used to investigate possible $\alpha$-cluster states in ${ }^{13} \mathrm{C}$. The reaction was measured at $25.5 \mathrm{MeV}$ incident energy, employing the São Paulo Pelletron-Enge-Spectrograph facility and the nuclear emulsion detection technique. Ten out of sixteen known levels of ${ }^{13} \mathrm{C}$, up to $11 \mathrm{MeV}$ of excitation, were observed and, due to the much improved energy resolution of $50 \mathrm{keV}$, at least three doublets could be resolved. This work presents a preliminary analysis of five of the most intensely populated states, also in comparison with the results of former transfer studies.
\end{abstract}

\section{Introduction}

The systematic study of $\alpha$-cluster spectroscopic strengths in odd-even light nuclei with $(x \alpha+v)$ structure is the main purpose of the investigation in progress. Experimentally, the $\alpha$-clustering phenomenon has been mainly studied through the $\left({ }^{6} \mathrm{Li}, \mathrm{d}\right)$ reaction on even-even nuclei [1] and, only a few works focused on odd-A nuclei. Referring to the $\alpha$-structure of ${ }^{13} \mathrm{C}$, data for the ${ }^{9} \mathrm{Be}\left({ }^{6} \mathrm{Li}, \mathrm{d}\right){ }^{13} \mathrm{C}$ reaction have been taken in São Paulo, using the Pelletron-Enge-Magnetic-Spectrograph facility, at an incident energy of $25.5 \mathrm{MeV}$. Calculations of the $\alpha$-cluster model, which does not consider internal excitations of the constituents of the $\alpha+{ }^{9} \mathrm{Be}$ system, are under way, aiming at generating alpha wave functions to be used in the DWBA description of the $\left({ }^{6} \mathrm{Li}, \mathrm{d}\right)$ reaction.

The former ${ }^{9} \mathrm{Be}\left({ }^{6} \mathrm{Li}, \mathrm{d}\right){ }^{13} \mathrm{C}$ works, by Gol'dberg et al. [2] and Aslanoglou et al. [3], presented energy resolutions of $400 \mathrm{keV}$ and $110 \mathrm{keV}$, respectively. In the present work the resolution of $50 \mathrm{keV}$ achieved contributes to a better understanding of the $\alpha+{ }^{9} \mathrm{Be}$ structure in ${ }^{13} \mathrm{C}$.

\section{Experimental Procedure}

The $25.5 \mathrm{MeV}{ }^{6} \mathrm{Li}$ beam of the São Paulo Pelletron accelerator was focused on a $131 \mu \mathrm{g} / \mathrm{cm}^{2}$, clean and uniform target of ${ }^{9} \mathrm{Be}$. The deuterons emerging from the $\left({ }^{6} \mathrm{Li}, \mathrm{d}\right)$ reaction were momentum analysed by the field of the Enge Magnetic Spectrograph and detected in nuclear emulsion plates (Fuji G6B, 50 $\mu \mathrm{m}$ thick). The plates covered $50 \mathrm{~cm}$ along the focal surface and spectra were measured at seven scattering angles, between $3^{\circ}$ and $20^{\circ}$ in the laboratory frame, spanning up to approximately $11 \mathrm{MeV}$ in ${ }^{13} \mathrm{C}$ excitation energies. After processing, the plates were scanned in strips of $200 \mu \mathrm{m}$ and an energy resolution of $50 \mathrm{keV}$ was achieved. Fig. 1 displays the deuteron spectrum corresponding to $\theta_{\text {lab }}=8^{\circ}$, showing the number of tracks per strip versus the position along the focal plane. In the figure, the excitation energies of ${ }^{13} \mathrm{C}$ in $\mathrm{MeV}$, taken from the systematics of Ajzenberg-Selove [4], associated with the deuteron peaks are indicated. A total of ten states, of the sixteen tabulated [4], was detected and the improvement of experimental conditions allowed for the separation of three doublets, corresponding respectively to the attributed ${ }^{13} \mathrm{C}$ excitation energies: $3.685 \mathrm{MeV}$ and $3.854 \mathrm{MeV}, 7.492$ $\mathrm{MeV}$ and $7.547 \mathrm{MeV}$, and $10.753 \mathrm{MeV}$ and $10.818 \mathrm{MeV}$. 


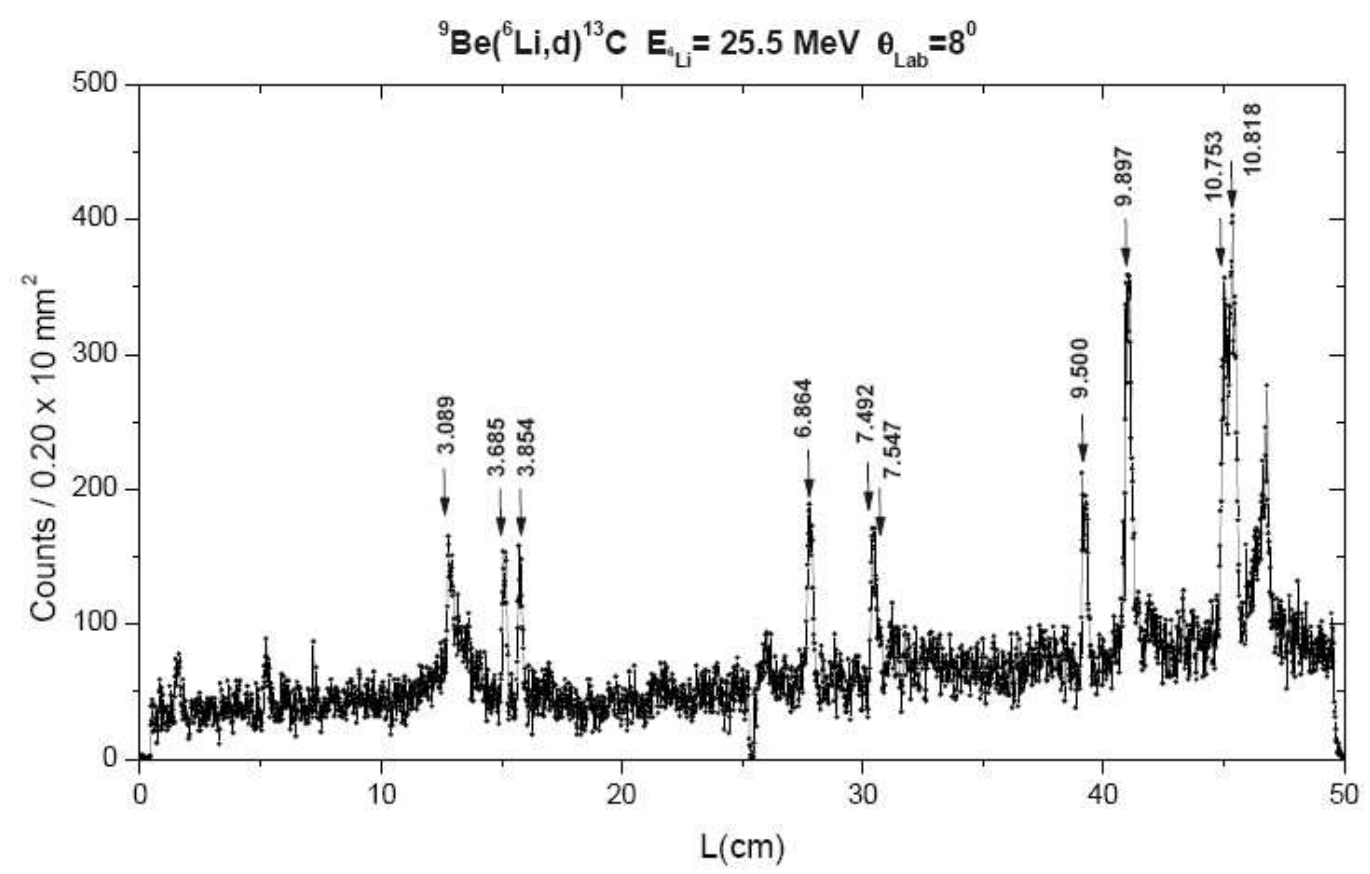

Fig. 1: Position deuteron spectrum. Indicated are the excitation energies from Ref. [4].

\section{Preliminary analysis and Results}

To describe the experimental angular distributions, particularly of the more intensely populated states, one step $\alpha$ transfer finite-range DWBA calculations using the code DWUCK 5 have been performed. In this preliminary analysis, the optical model description for the entrance channel $\left({ }^{9} \mathrm{Be}+{ }^{6} \mathrm{Li}\right)$ took the global parameter set of Cook [5], with a slight decrease [6] in the geometrical parameters, as indicated by the fit of the elastic scattering angular distribution of ${ }^{6} \mathrm{Li}$ in ${ }^{13} \mathrm{C}$, measured with the same incident energy. It is to be remembered that the optical potential for the entrance channel is quite important since it defines the door-way state of the transfer reaction. The exit channel $\left(\mathrm{d}+{ }^{13} \mathrm{C}\right)$ optical potential applied was that of Daehnick et al. [7] and for the $(\alpha+\mathrm{d})$ description of ${ }^{6} \mathrm{Li}$ the Kubo and Hirata [8] binding potential was taken. A Woods-Saxon binding potential for the $\left(\alpha+{ }^{9} \mathrm{Be}\right)$ system, with reduced radius of $1.25 \mathrm{fm}$ and diffuseness of $0.65 \mathrm{fm}$ was applied, the depth being adjusted to reproduce the binding energy of each ${ }^{13} \mathrm{C}$ state. The number of nodes $N$ of the transferred $\alpha$ particle radial wave function and, the orbital angular momentum $L$, relative to the ${ }^{9} \mathrm{Be}$ core, were determined by the oscillatory energy conservation relation $G=2(N-1)+L=\sum_{i}\left[2\left(n_{i}-1\right)+l_{i}\right]$, where $\left(n_{i}, l_{i}\right)$ are the single nucleon shell quantum numbers. In the present work a $(1 \mathrm{p})^{4}$ single particle configuration was assumed for the negative parity states $(G=4)$ and for the positive parity states a $(1 \mathrm{p})^{3}(1 \mathrm{~d})$ structure $(G=5)$ was considered.

The known states [4] at $3.685 \mathrm{MeV}\left(3 / 2^{-}\right)$and at $3.854 \mathrm{MeV}\left(5 / 2^{+}\right)$, seen as doublet in former $\alpha$ transfer studies [2,3], are well resolved in the present work. Fig. 2 shows the corresponding experimental angular distributions in comparison with DWBA predictions. The angular distribution associated with the $3 / 2^{-}$state needs an $L=0+2$ mixture to be reproduced, since, due to the experimentally observed filling of the predicted minimum, a pure $L=0$ contribution as indicated by Aslanoglou et al. [3] is not sufficient. In the case of the $5 / 2^{+}$state at $3.854 \mathrm{MeV}$, which could be reached through $L=1$ and $L=3$ transfers, the $L=1$ dominates. 


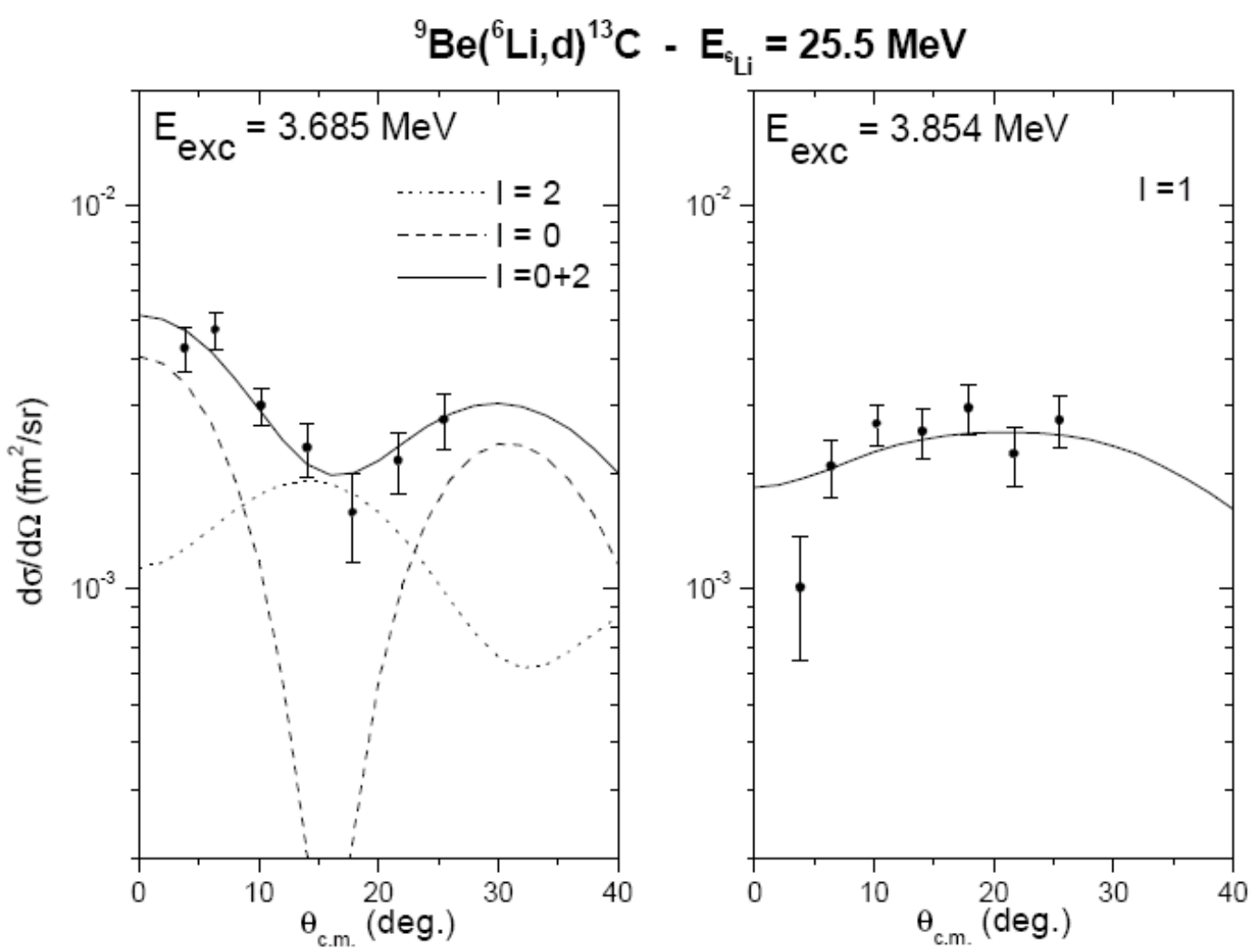

Fig. 2: Experimental angular distributions in comparison with DWBA predictions for the states at $3.685 \mathrm{MeV}\left(3 / 2^{-}\right)$and at $3.854 \mathrm{MeV}\left(5 / 2^{+}\right)$.

The experimental angular distributions and DWBA predictions for the most intensely populated states, the doublet at $10.8 \mathrm{MeV}$, now resolved, and the state $3 / 2^{-}$at $9.897 \mathrm{MeV}$, are presented in Fig. 3. The DWBA analysis for the states $7 / 2^{-}$at $10.753 \mathrm{MeV}$ and $\left(5 / 2^{-}\right)$at $10.818 \mathrm{MeV}$ assumed both bound by $100 \mathrm{keV}$, although they are unbound. An almost pure $L=2$ transfer can describe the experimental angular distribution of the $3 / 2^{-}$state, even if an admixture of $L=0$, as also tried by Aslanoglou et al. [3], could improve the fit somewhat. As was the case also for several other experimental angular distributions, the analysis of the previous work [3] was unable to reproduce their data, the structure of the data distribution being, at least, out of phase with the prediction. The integrated experimental angular distribution associated with the states $7 / 2^{-}$and $\left(5 / 2^{-}\right)$in the former work [3] was fitted by a pure $L=2$ transfer. In the present work, for both transitions, a pure $L=4$ transfer is indicated instead.

According to Millener et al. [9], who performed a detailed investigation of electron inelastic scattering on ${ }^{13} \mathrm{C}$, and in agreement with ${ }^{13} \mathrm{C}$ shell model calculations [10], the three states, $3 / 2^{-}, 7 / 2^{-}$ and $\left(5 / 2^{-}\right)$, under analysis present predominantly a $(1 \mathrm{~s})^{4}(1 \mathrm{p})^{7}(2 \mathrm{~s} 1 \mathrm{~d})^{2}$ configuration, involving, therefore, components above the $\mathrm{p}$ shell. For the three states mentioned, the angular distributions and DWBA fits, considering the $(1 \mathrm{p})^{2}(2 \mathrm{~s} 1 \mathrm{~d})^{2}$ single particle configuration and $G=6$ for the transferred alpha, would only result in lower spectroscopic intensities, without any pronounced difference in shape of the predicted angular distributions.

The experimental angular distributions of the states $3 / 2^{-}(9.897 \mathrm{MeV})$ and $7 / 2^{-}(10.753 \mathrm{MeV})$ were also compared in Fig. 3 with the DWBA predictions using the form factor described by the radial wave functions taken from Souza and Miyake calculations [11]. The local cluster-core potential for the $\alpha+{ }^{9} \mathrm{Be}$ system uses the nuclear term based on the form proposed by Buck, Merchant and Perez [12], 
adding Coulomb and spin-orbit terms. A good agreement with the data was obtained specially for the $7 / 2^{-}$state, which is associated in the calculation with an $L=4$ angular momentum.

Intense resonances [4] in the neutron elastic scattering on ${ }^{12} \mathrm{C}$ can be associated with the states in ${ }^{13} \mathrm{C}$ which are excited in the $\alpha$ transfer, at $10.753 \mathrm{MeV}$ and $10.818 \mathrm{MeV}$, slightly above the ${ }^{9} \mathrm{Be}+\alpha$ threshold, possibly with astrophysical implications.

The results here presented are still preliminary, in the short term the $\alpha+{ }^{9} \mathrm{Be}$ wave functions calculated [11] will be used in the DWBA descriptions to extract the $\alpha$ spectroscopic strengths of the most intensely excited states. Next, the influence on the DWBA predictions of the full complex remnant term [13] inclusion in the residual interaction will be investigated.

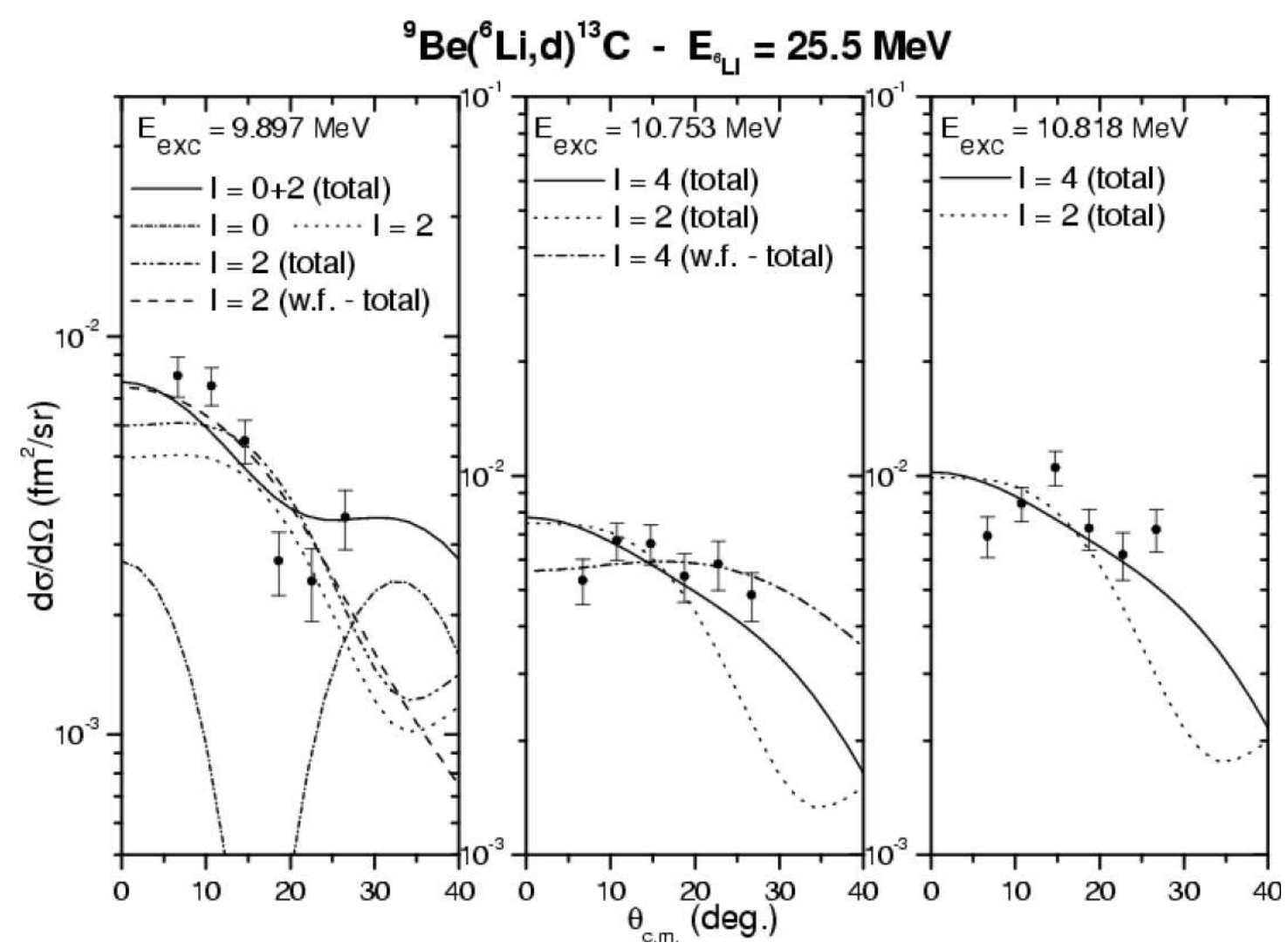

Fig. 3: Experimental angular distributions in comparison with DWBA predictions for the most intensely populated states. The results using the wave functions (w.f.) from Ref. [11] for the states $9.897 \mathrm{MeV}$ and 10.753 $\mathrm{MeV}$ are also indicated.

\section{Acknowledgments}

This work was partially supported by the brazilian funding agencies FAPESP and CAPES.

\section{References}

[1] H.W. Fulbright et al., Nucl. Phys. A284, 329 (1977). 
[2] V.Z. Gol'dberg et al., Izv. Akad. Nauk SSSR, Ser. Fiz. 35, 1663 (1971); Bull. Acad. Sci. USSR, Phys. Ser. 35, 1514 (1972).

[3] X. Aslanoglou et al., Phys. Rev. C 40, 73 (1989).

[4] F. Ajzenberg-Selove, Nucl. Phys. A523, 1 (1991).

[5] J. Cook, Nucl. Phys. A388, 153 (1982).

[6] M.R.D. Rodrigues et al., Proceedings of the XXX Reunião de Trabalho sobre Física Nuclear no Brasil, 40 (2007).

[7] W.W. Daehnick, J.D. Childs and Z. Vrcelj, Phys. Rev. C 21, 2253 (1980).

[8] K.I. Kubo and M. Hirata, Nucl. Phys. A187, 186 (1972).

[9] D. J. Millener et al., Phys. Rev. C 39, 14 (1989).

[10] J. F. Dubach, Los Alamos Scientific Laboratory Report 2A 8303-C1980, p. 72.

[11] M.A. Souza and H. Miyake, Proceedings of the XXX Reunião de Trabalho sobre Física Nuclear no Brasil, 46 (2007).

[12] B. Buck, A.C. Merchant and S.M. Perez, Phys. Rev. C 51, 559 (1995).

[13] N. Keeley, K.W. Kemper, Dao T. Khoa, Nucl. Phys. A726, 159 (2003). 


\section{Referências Bibliográficas}

[1] B. Buck, C.B. Dover and J.P. Vary, Phys. Rev. C 11, 1803 (1975).

[2] F. Michel, G. Reidemeister and S. Ohkubo, Phys. Rev. C 37, 292 (1988).

[3] B. Buck, A.C. Merchant and S.M. Perez, Phys. Rev. C 51, 559 (1995).

[4] S. Ohkubo, Phys. Rev. Lett. 74, 2176 (1995).

[5] F. Michel, G. Reidemeister and S. Ohkubo, Phys. Rev. C 61, 041601(R) (2000).

[6] M.A. Souza and H. Miyake, Braz. J. Phys. 35, 826 (2005).

[7] F. Hoyler, P. Mohr and G. Staudt, Phys. Rev. C 50, 2631 (1994).

[8] B. Buck and A.A. Pilt, Nucl. Phys. A280, 133 (1977).

[9] H. Furutani et al., Suppl. Prog. Theor. Phys. 68, 193 (1980).

[10] A.C. Merchant, Nucl. Phys. A417, 109 (1984).

[11] B. Buck, H. Friedrich and A.A. Pilt, Nucl. Phys. A290, 205 (1977).

[12] B. Buck, A.C. Merchant and N. Rowley, Nucl. Phys. A327, 29 (1979).

[13] Y. Fujiwara et al., Suppl. Prog. Theor. Phys. 68, 29 (1980).

[14] F. Michel, S. Ohkubo and G. Reidemeister, Prog. Theor. Phys. Suppl. 132, 7 (1998).

[15] T. Sakuda and S. Ohkubo, Prog. Theor. Phys. Suppl. 132, 103 (1998).

[16] A.C. Merchant, Phys. Rev. C 36, 778 (1987).

[17] P. Mohr, The Open Nucl. \& Particle Phys. Journal 1, 1 (2008).

[18] G. Audi, A.H. Wapstra and C. Thibault, Nucl. Phys. A729, 337 (2003).

Referência na Internet: http://www.nndc.bnl.gov/amdc/ 
[19] K. Wildermuth and Y.C. Tang, A Unified Theory of the Nucleus (Academic Press, New York, 1977).

[20] K. Wildermuth and Th. Kanellopoulos, Nucl. Phys. 7, 150 (1958).

[21] G.R. Satchler, Direct Nuclear Reactions (Oxford University Press, New York, 1983).

[22] B. Buck et al., Phys. Rev. C 52, 1840 (1995).

[23] B. Buck, A.C. Merchant and S.M. Perez, Nucl. Phys. A657, 267 (1999).

[24] B. Buck, A.C. Merchant and S.M. Perez, Few-Body Systems 29, 53 (2000).

[25] W.S.C. Willians, Nuclear and Particle Physics (Clarendon Press - Oxford, New York, 1991), p. 139.

[26] A. Bohr and B.R. Mottelson, Nuclear Structure, vol. 1 (W.A. Benjamin, Inc., New York, 1969), p. 224.

[27] S.G. Nilsson and I. Ragnarsson, Shapes and Shells in Nuclear Structure (Cambridge University Press, Cambridge, 1995), p. 60.

[28] S.S.M. Wong, Introductory Nuclear Physics (Wiley-VCH Verlag GmbH \& Co. KGaA, Weinheim, 2004), p. 245.

[29] R. Eisberg e R. Resnick, Física Quântica (Campus, Rio de Janeiro, 1979), p. 676.

[30] B. Singh and A.R. Farhan, Nucl. Data Sheets 107, 1923 (2006).

Referência na Internet: http://www.nndc.bnl.gov/chart/

[31] P.L. DeVries, A First Course in Computational Physics (John Wiley \& Sons, Inc., Oxford, 1994).

[32] J.L. Powell and B. Crasemann, Quantum Mechanics (Addison-Wesley Publishing Company, Estados Unidos, 1961).

[33] Marco Antonio de Souza, O modelo de Cluster-alfa Aplicado ao ${ }^{94} \mathrm{Mo}$ (Dissertação de Mestrado, Universidade de São Paulo, Instituto de Física, 2005).

[34] S. Gasiorowicz, Física Quântica (Guanabara Dois, Rio de Janeiro, 1979).

[35] S. Ohkubo, Phys. Rev. C 38, 2377 (1988).

[36] U. Atzrott et al., Phys. Rev. C 53, 1336 (1996). 
[37] E. Browne, Nucl. Data Sheets 82, 379 (1997).

[38] C.M. Baglin, Nucl. Data Sheets 91, 423 (2000).

[39] B.A. Brown et al., Phys. Rev. C 14, 602 (1976).

[40] D. Abriola and A.A. Sonzogni, Nucl. Data Sheets 107, 2423 (2006).

[41] H. Bohn et al., Phys. Rev. Lett. 29, 1337 (1972).

[42] D. Abriola and A.A. Sonzogni, Nucl. Data Sheets 109, 2501 (2008).

[43] B. Singh and Z. Hu, Nucl. Data Sheets 98, 335 (2003).

[44] D.R. Tilley et al., Nucl. Phys. A636, 249 (1998).

[45] J.A. Cameron and B. Singh, Nucl. Data Sheets 88, 299 (1999).

[46] E. Browne, Nucl. Data Sheets 104, 427 (2005).

[47] I. Angeli, At. Data and Nucl. Data Tables 87, 185 (2004).

[48] A. Arima and S. Yoshida, Nucl. Phys. A219, 475 (1974).

[49] G. Michaud, L. Scherk and E. Vogt, Phys. Rev. C 1, 864 (1970).

[50] H. Horiuchi and Y. Suzuki, Prog. Theor. Phys. 49, 1974 (1973).

[51] N. Keeley, K.W. Kemper and D.T. Khoa, Nucl. Phys. A726, 159 (2003).

[52] F.D. Becchetti, J. Jänecke and C.E. Thorn, Nucl. Phys. A305, 313 (1978).

[53] Chang-hua Zhang, Shun-jin Wang and Jin-nan Gu, Phys. Rev. C 60, 054316 (1999).

[54] T. Yamaya et al., Prog. Theor. Phys. Suppl. 132, 73 (1998).

[55] A. Messiah, Quantum Mechanics, vol. 1 (John Wiley \& Sons, Inc., New York, 1976).

[56] B. Buck, A.C. Merchant and S.M. Perez, Nucl. Phys. A652, 211 (1999).

[57] B. Buck, A.C. Merchant and S.M. Perez, Nucl. Phys. A673, 157 (2000).

[58] S. Ohkubo and K. Yamashita, Phys. Rev. C 66, 021301(R) (2002).

[59] M.A. Souza and H. Miyake, Proceedings of the XXX Reunião de Trabalho sobre Física Nuclear no Brasil (Águas de Lindóia, São Paulo), editado por A. Suaide (SBF, São Paulo, 2008), pág. 46. 
[60] M.R.D. Rodrigues et al., Proceedings of the $12^{\text {th }}$ International Conference on Nuclear Reaction Mechanisms (Varenna, Italy), editado por F. Cerutti e A. Ferrari (CERN, Genebra, 2010), pág. 331. 